\title{
A Qualitative Study of Medication Adherence amongst People with Schizophrenia
}

\author{
By Lucinda Jane Clifford \\ Thesis submitted for the degree of \\ Combined Master of Psychology (Clinical) / Doctor of Philosophy \\ School of Psychology \\ The University of Adelaide
}

December, 2012 


\section{Table of Contents}

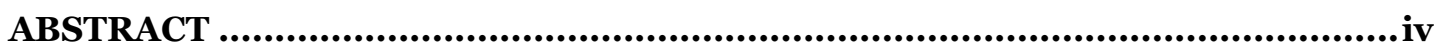

DECLARATION ........................................................................................vi

ACKNOWLEDGEMENTS ...........................................................................vii

Chapter 1: Schizophrenia ................................................................................... 1

1.1 Symptoms according to the medical model and alternative ways of understanding .. 2

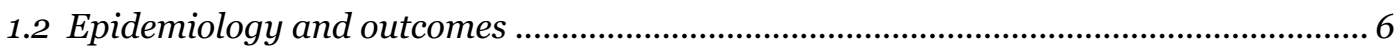

1.3 Stages of recovery ....................................................................................................... 10

1.4 Chapter summary .......................................................................................................

Chapter 2: Treatments for Schizophrenia ............................................ 13

2.1 Antipsychotic medication nomenclature ........................................................................

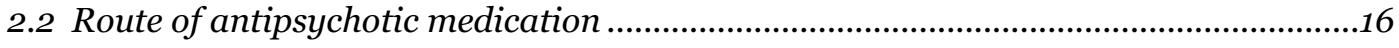

2.3 How antipsychotic medications work in brief .......................................................17

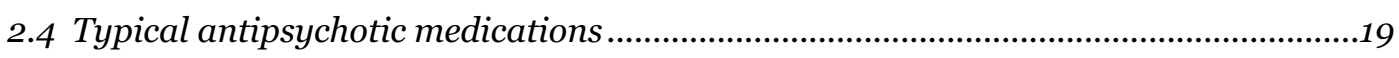

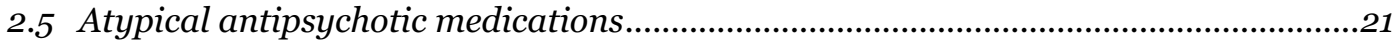

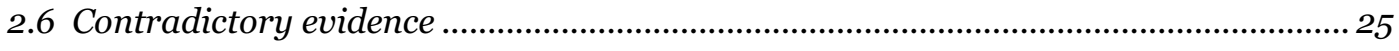

2.7 Summary of indications for typical and atypical medications, different routes of

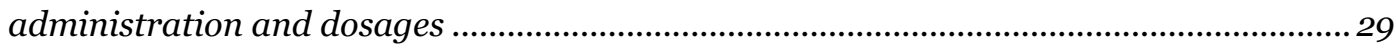

2.8 Continuous maintenance pharmacotherapy ................................................................

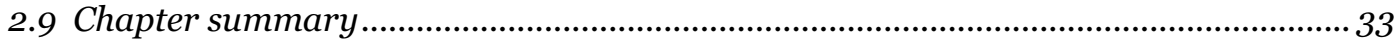

Chapter 3: Medication Adherence .....................................................................36

3.1 Terminology ................................................................................................................ 37

3.2 Impact of adherence on symptoms and relapse ................................................................. 39

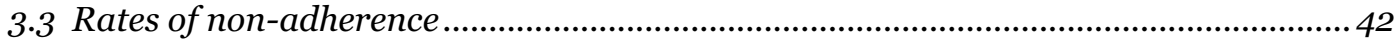

3.4 Factors proposed to influence adherence ………............................................................ 45

3.4.1 The Rating of Medication Influences Scale ..............................................................4 46

3.4.2 Literature reviews ............................................................................................... 47

3.4.4 Qualitative research related to adherence …..........................................................51

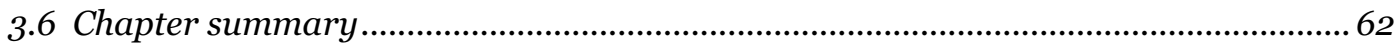

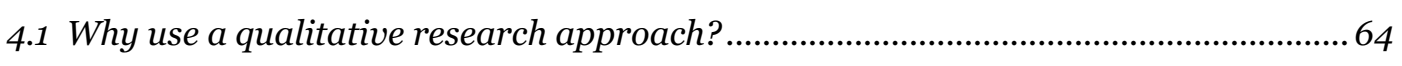

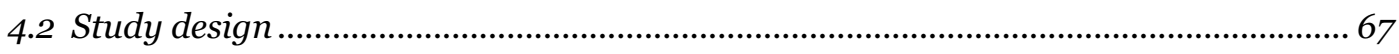

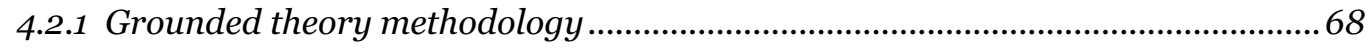

4.2.2 Ethical considerations .........................................................................................

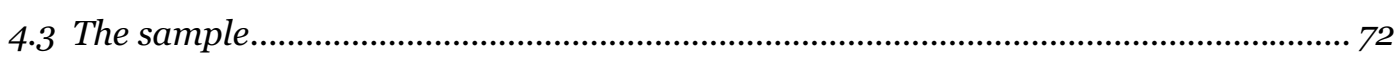

4.3.1 Recruitment strategy and sampling frame ……................................................. 72

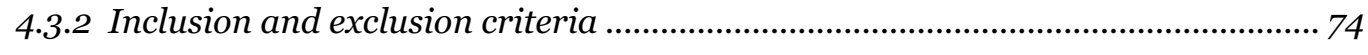

4.3.3 Selection of participants ......................................................................................... 75

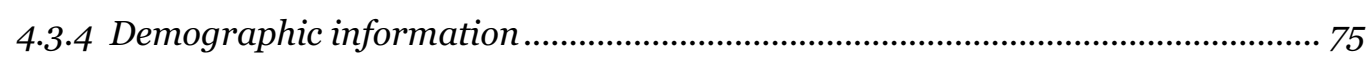




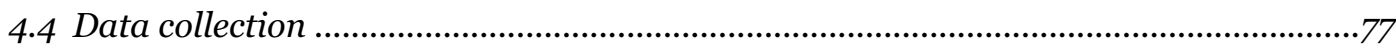

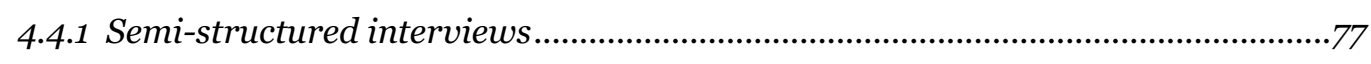

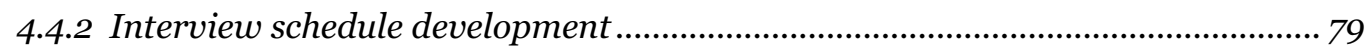

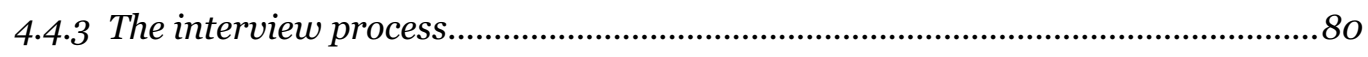

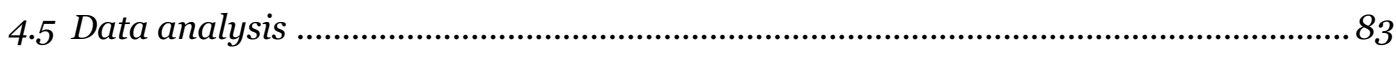

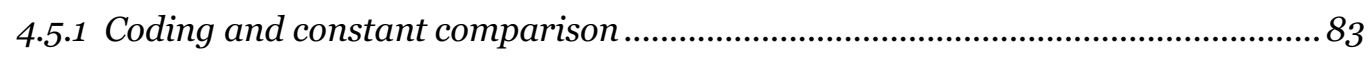

Chapter 5: Consumer-related factors ....................................................86

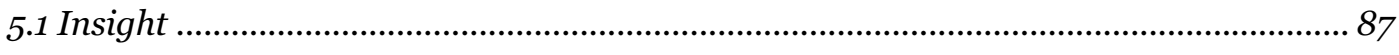

5.1.1 Awareness of having an illness ...........................................................................8 88

5.1.2 Awareness of the risk of relapse ..................................................................... 97

5.2 Reflection on experiences - how insight is gained .................................................112

5.2.1 Experiences of illness prior to medication treatment .........................................114

5.2.2 Experiences of the consequences of non-adherence............................................116

5.2.3 Experiences of the benefits of medication ............................................................121

5.2.4 Observations of other consumers ....................................................................123

5.2.5 Interventions that promote reflection .............................................................. 126

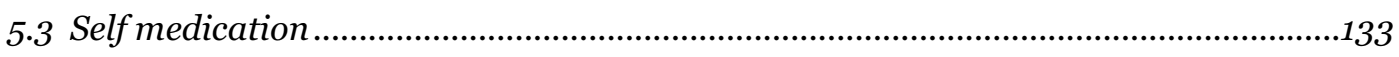

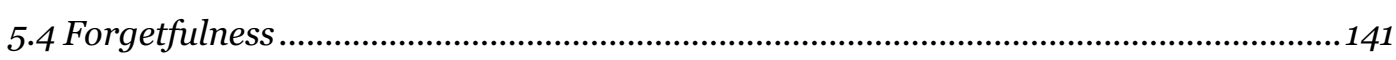

5.4.1 Daily routine ................................................................................................ 143

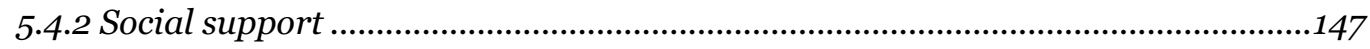

5.5 Chapter summary .......................................................................................... 150

Chapter 6: Medication-related Factors......................................................... 153

6.1 The route of administration ..................................................................................155

6.2 Storage of medication ........................................................................................... 158

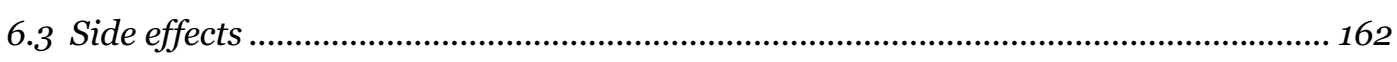

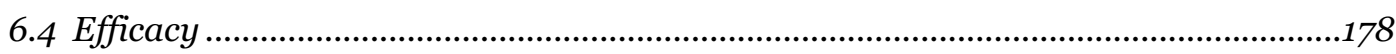

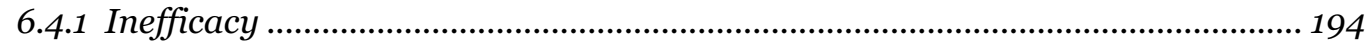

6.5 Consideration of side effects and efficacy collectively ............................................ 199

6.6 Chapter summary ..................................................................................................208

Chapter 7: Service-related factors ........................................................210

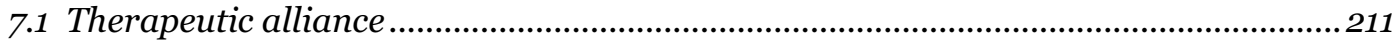

7.1.1 Power relations in the therapeutic alliance: collaboration vs. authoritarianism

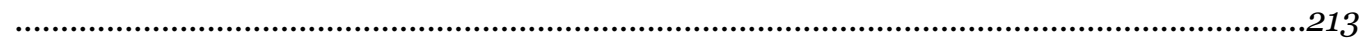

7.1.2 Interest in the consumer's experiences and background knowledge ..................224

7.1.3 Tailoring the medication regimen to the consumer ...........................................229

7.1.4 Non-adherence as resistance .......................................................................245

7.2 Community centres and peer workers ................................................................250

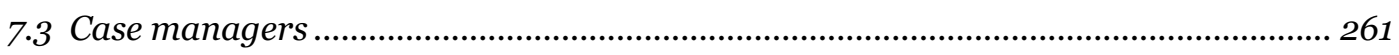

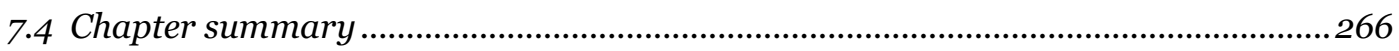

Chapter 8: Discussion ........................................................................269 
8.1 Interview experiences: support for greater inclusion of the consumer perspective in future research

8.2 The sample and generalisability of findings …………................................................271

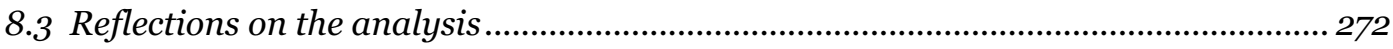

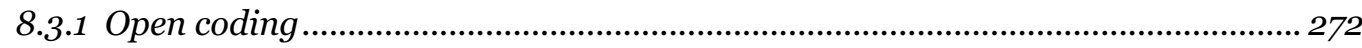

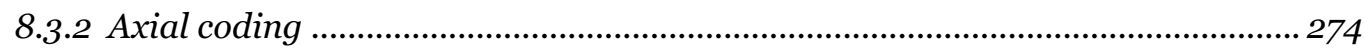

8.3.2.1 Consumer-related factors category ………................................................... 277

8.3.2.2 Medication-related factors category ................................................................284

8.3.2.3 Service-related factors category ........................................................................28

8.4 Potential clinical implications ……………................................................................289

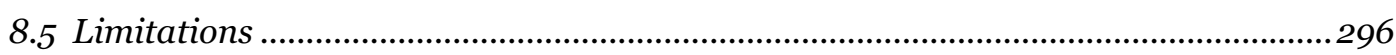

8.6 Considerations for future research ………..................................................................2 297

8.7 Contribution and significance ……………...............................................................298

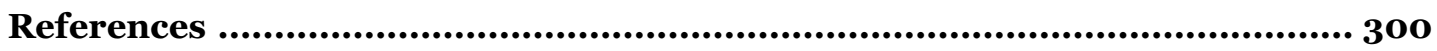

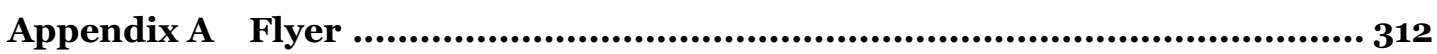

Appendix B Interview Schedule ................................................................. 313

Appendix C Information Sheet ................................................................. 314

Appendix D Consent Form ...........................................................................316

Appendix E Table of Demographic Information ....................................... 317

\section{Tables}

Table 1: Risk factors for non-adherence to antipsychotic medications and follow-up appointments amongst people with schizophrenia (from Compton, 2007). 


\begin{abstract}
The introduction of antipsychotic medication revolutionised the treatment of schizophrenia and significantly improved outcomes for consumers. Nonetheless, non-adherence to antipsychotic medication regimens is common amongst consumers. Whilst ample research has attempted to quantify rates of adherence and identify influences on adherence and non-adherence, few qualitative studies have been undertaken in the area and the consumers' voices have thereby been limited. The research presented in this thesis aimed to enhance understanding of medication adherence from the consumer perspective. Qualitative, semistructured, one-to-one interviews were conducted with 25 outpatients with schizophrenia from metropolitan Adelaide. Interviews were audio-recorded, transcribed and analysed, guided by a grounded theory approach. Codes identified in open coding were grouped into categories, reflective of the different aspects of consumers' medication taking experiences. It is argued that consumer-related factors, medication-related factors and service-related factors influence adherence behaviour. Whilst some of the codes that were identified as influences on adherence were consistent with previous research findings, such as insight, side effects, efficacy and therapeutic alliance, analysis elaborated different aspects of these factors and shed some light on how they influence adherence. The most commonly raised, novel codes that emerged from interview data related to reflection on experiences and peer worker intervention. Specifically, interviewees commonly reported that they learned from previous illness and medication-related experiences, including


the experience of non-adherence, which was frequently constructed as a motivator for future adherence. Furthermore, when enquired about interventions, many interviewees suggested that peer workers may work more effectively with consumers to encourage adherence. In particular, peer workers were were positioned as having more credibility than other service providers due to their shared experiences with consumers. Research findings support greater involvement of consumers in research due to their valuable contributions. Furthermore, regarding the clinical implications, findings support tailored, individualised interventions, enhanced peer worker involvement and challenge service providers' poor tolerance of nonadherence on the grounds that adherence may represent a learning process. 


\section{DECLARATION}

I certify that this work contains no material which has been accepted for the award of any other degree or diploma in any university or other tertiary institution to Lucinda Clifford and, to the best of my knowledge and belief, contains no material previously published or written by another person, except where due reference has been made in the text. In addition, I certify that no part of this work will, in the future, be used in a submission for any other degree or diploma in any university or other tertiary institution without the prior approval of the University of Adelaide.

I give my consent to this copy of my thesis, when deposited in the University Library, being made available for loan and photocopying, subject to the provisions of the Copyright Act 1968.

I also give permission for the digital version of my thesis to be made available on the web, via the University's digital research repository, the Library catalogue, and also through web search engines, unless permission has been granted by the University to restrict access for a period of time.

Lucinda Clifford

Date 


\section{ACKNOWLEDGEMENTS}

I'd like to take the opportunity to thank those who have been instrumental in assisting me throughout the course of my research journey.

Firstly, to my supervisory panel, Professor Deborah Turnbull, Dr Shona Crabb and Professor Cherrie Galletly - thank you all for your perseverance, guidance and support. Deb, you have consistently provided me with constructive feedback, motivation and gave me a push when I needed it! Shona, your encouragement and positive regard have been much appreciated and your analytical knowledge has been invaluable. Cherrie, your extensive clinical experience combined with your enthusiasm for the subject matter was a true inspiration. I'd also like to mention Bev Hisee, Research Nurse, who assisted me greatly in my interactions with consumers and whose knowledge and genuineness impressed.

To the incredible group of interviewees involved in this research and to those consumers who I met along the way - this thesis would not have been possible without your insightful contributions. Thank you for your openness, cooperation and for your fascinating stories that have brought this thesis to life.

To my family, in particular, my parents, Joe and Carol, thanks for putting up with me and for putting a roof over my head for all these years. I promise to clear the mound of articles that have accumulated in the study! You have always been there for me to rely on and I cannot thank you enough for your ongoing belief in me. To my beautiful sister, Rebecca, my brotherin-law, Josh, and the adorable Moll - thank you for opening up your home to me and for giving me perspective during the tough times. Josh, not sure what I would have done without your last-minute technical support! 
Finally, I'd like to acknowledge my fabulous friends for all the laughs and good times over the years that have kept me going. 


\section{Chapter 1: Schizophrenia}

Schizophrenia is a serious mental illness which is often associated with significant implications for people's quality of life. The extent to which individuals with diagnoses of schizophrenia adhere to their antipsychotic medications is considered an important influence on their outcomes. Whilst medication adherence amongst people with schizophrenia has been studied extensively, the majority of research has been quantitative and thus, the voices of consumers have largely been neglected. One reason that has been proposed for this absence is the assumption that people with schizophrenia would not be able to provide meaningful contributions to knowledge. This thesis aims to redress the dearth of consumers' voices in adherence research by examining their perspectives through qualitative interviews. Analysis of interview data supports the significant value of the inclusion of consumers' voices in research to enhance understanding of medication adherence.

In order to be included in the research presented in this thesis, participants were required to meet the Diagnostic and Statistical Manual of Mental Disorders, Fourth Edition, Text Revision (DSM-IV-TR) (American Psychiatric Association, 2000) criteria for schizophrenia. According to Freedman (2005), schizophrenia is a chronic disability of mental and social function, with superimposed, recurrent episodes of exacerbated psychotic symptoms, such as delusions and/or hallucinations. Despite being considered one of the most severe, disabling and economically draining mental illnesses (Picchioni \& Murray, 2007), Schneider (2010) points out that people diagnosed with schizophrenia can and do participate in valued social roles and lead satisfying, productive lives, consistent with research on 
recovery in schizophrenia (Liberman \& Kopelowicz, 2005; Resnick, Rosenheck \& Lehman, 2004). This chapter will summarise the symptoms of schizophrenia according to the medical model. This is followed by a discussion of the social constructionist position as an alternative perspective for understanding mental illness and schizophrenia in particular. An understanding of what schizophrenia is and the epidemiology of schizophrenia has been included in an attempt to contextualise the sample of interviewees, by describing the accepted view of what people with schizophrenia in the general population experience in terms of illness symptoms as well as the associated outcomes. Critically, some of the unsettling statistics regarding the significant impact that schizophrenia has on the lives of consumers and the community reinforce the benefits of research aimed at improving the outcomes for people with schizophrenia.

\subsection{Symptoms according to the medical model and alternative ways of understanding}

Symptoms of schizophrenia can be understood as the consequences of disordered cognition and emotion, which manifest as disturbances in language, thought, perception, affect and sense of self (McGorry, 1992). A clinical diagnosis of schizophrenia requires the presence of delusions and/or hallucinations, formal thought disorder and unusual behaviour lasting for at least one month, with significant social and occupational deterioration experienced prior or subsequent to psychotic symptoms (Picchioni \& Murray, 2007; Sharif, Bradford, Stroup \& Lieberman, 2007). DSM-IV-TR criteria also require six months of continuous symptoms to be exhibited including at least one week of psychotic symptoms (American Psychiatric Association, 
2000). People with a diagnosis of schizophrenia typically experience symptoms which are consistently described by the dominant medical model of clusters of positive, negative and cognitive symptoms. However, some individuals may predominantly experience symptoms from positive or negative clusters, respectively (Cutting, 2003). Positive symptoms are so called because they are considered an addition to a person's repertoire (Birchwood \& Jackson, 2001). Positive symptoms include things such as delusions, unusual thoughts and suspiciousness, paranoia, hallucinations and distorted perceptions typically considered to be manifestations of psychosis (McEvoy, Scheifler \& Frances, 1999). Negative symptoms are those that are evident by the blunting of motivation and emotion; for example, social withdrawal, lack of energy, loss of sense of pleasure, inability to make decisions, limited speech and poor self care (Smith, Weston \& Lieberman, 2009). Negative symptoms persist even in the absence of positive symptoms during periods of remission; however, they may be secondary to other factors, such as depression (McGorry, 1992). Cognitive symptoms common to people with schizophrenia include problems with attention, learning new information, memory, verbal fluency, problem solving, recognising social cues, confused thinking, disorganised speech and disorganised behaviour (Freedman, 2005; McEvoy et al., 1999; Sharif et al., 2007). It is estimated that approximately $75 \%$ of people with schizophrenia have clinically meaningful deficits in at least two cognitive domains and 90\% have deficits in one (Sharif et al., 2007).

An alternative medical model of schizophrenia that is also often deployed by researchers is comprised of clusters of positive, negative and disorganised symptoms (Beck, Rector, Stolar \& Grant, 2009; Cutting, 2003; Sharif et al., 2007). Such models typically group cognitive impairments (such 
as impairments in attention, learning, memory and perception) in the negative symptom cluster. Disorganisation symptoms include disordered thought processes, bizarre behaviour and disturbances of emotions or inappropriate affect (Cutting, 2003; Sharif et al., 2007).

The DSM-IV-TR includes five sub-classifications to account for variations in presentations amongst people with schizophrenia. Specifically, the paranoid type describes individuals who experience delusions (persecutory or grandiose) or hallucinations but thought disorder, disorganised behaviour and negative symptoms are absent. Individuals are diagnosed as the disorganised type when they present with both thought disorder and flattened affect. The catatonic type defines those who exhibit agitated, purposeless movement or are immobile. The undifferentiated type is diagnosed in cases when psychotic symptoms are present but do not meet criteria for the paranoid, disorganised or catatonic types. The residual type is diagnosed when individuals experience mild positive symptoms only.

The above descriptions of schizophrenia are based on the current, dominant construction of schizophrenia as a mental illness or pathology, in line with psychiatry's medical model. Schneider (2010) highlights the fact that schizophrenia has not always regarded as an illness in line with the current dominant medical model of health, as the ever-changing historical accounts of schizophrenia or "madness" indicate.

There is also a significant social constructionist literature which suggests that "schizophrenia" is but a disease metaphor which has gained acceptance as a bio-psychiatric entity despite a lack of evidence (Wise, 2004). By a lack of evidence, researchers allude to an absence of medical tests to confirm a diagnosis, no clear causes identified and there being no consistent set of symptoms present in all cases (Schneider, 2010). Advocates of this 
position dispute the use of diagnostic labels to describe people's experiences and regard terminology such as "schizophrenia" and "mental illness" as mechanistic social constructions of deviant behaviour which are morally based and serve stigmatising functions (Schneider, 2010; Wise, 2004). The medical management of what is, ultimately, considered to be unwanted conduct according to some moral standard is, thus, also frequently contested by proponents of a social constructionist position (Wise, 2004).

Whilst I acknowledge the social constructionist position and am sympathetic towards it, I use the terms 'schizophrenia' and 'mental illness' throughout this thesis. Indeed, I also screened participants in the current study using an instrument based on DSM-IV criteria for schizophrenia. The term 'schizophrenia' has been used to describe people who have been given a diagnosis of the illness from a mental health professional based on meeting the present criteria of the current medical model of schizophrenia. As Schneider (2010, p18) states:

"While I acknowledge the difficulties of using the word schizophrenia, our research is an attempt to change the meanings of this word by demonstrating the ability of people diagnosed with schizophrenia to make a significant contribution to knowledge about schizophrenia".

I refer to people with schizophrenia as "consumers" throughout the present study, to reflect the interaction between individuals and the health system. "Consumer" terminology conveys the idea that individuals who are receiving psychiatric services should have choices and participate in decisionmaking (Torrey, 2011). Whilst this reflects more politically correct terminology, the results of the present study indicate that the term "consumer" may not adequately describe the interaction between people with 
schizophrenia and the health system, which in actuality is often entrenched with power imbalances in the favour of service providers(this interaction will be elaborated further in Chapter 7). It is acknowledged that some commentators have criticised use of the term "consumers" as a descriptor, as it implies that people with schizophrenia are aware that they have an illness and are thus, able to make treatment choices, which may not be the case amongst people who lack insight (i.e., Torrey, 2011).

\subsection{Epidemiology and outcomes}

Whilst the causes of schizophrenia remain unclear, risk factors that have been reported in the literature include a family history, obstetric complications, developmental difficulties, childhood central nervous system infections, cannabis use and acute life events. Of note, there is a lack of consensus regarding the contributions of each of these risk factors and how they interact (Smith et al., 2009). The lifetime prevalence rate of schizophrenia has been estimated at approximately $0.7 \%-1 \%$ of the population (Beck et al., 2009; Sharif et al., 2007). Although these figures may appear modest, schizophrenia rates among the top ten causes of longterm disability in the world (Compton, 2007). The onset of schizophrenia symptoms typically occurs in adolescence and early adulthood (average age 25 years), although it can affect people at any age (Smith et al., 2009; Van Os \& Kapur, 2009).

The epidemiology for schizophrenia has undergone a major shift in the past decade (Beck et al., 2009; Menezes, 2009). The prevailing view of the 1980 and 1990 s was that schizophrenia occurs at similar rates in all populations around the world, irrespective of individual or group 
characteristics such as gender and culture. This accepted notion rendered epidemiological studies seeking to identify risk factors for schizophrenia negligible as such studies would require heterogeneity in prevalence between groups and populations (Menezes, 2009). A recent renewed interest in the epidemiology of schizophrenia, especially in relation to incidence and outcomes, in conjunction with results from systematic reviews in the field have led to the replacement of received notions about schizophrenia with a more nuanced perspective.

A systematic review conducted by McGrath et al. (2004) is highly regarded as representing a critical turning point in the revived interest in the epidemiology of schizophrenia. This systematic review yielded wide variation in the incidence rates of schizophrenia across populations, regions and groups. Namely, it was found that incidence rates vary considerably crossculturally, with a fivefold variation observed between central estimates. Males are at greater risk of developing schizophrenia than women. The gender ratio (male:female) observed was 4:1 (McGrath et al., 2004). Males also tend to develop schizophrenia earlier in life than females (Beck et al., 2009). Furthermore, it has been reported that males experience more severe symptoms, more negative symptoms, have less chance of recovery and have generally worse outcomes than females (Picchioni \& Murray, 2007). The incidence of schizophrenia appears to be declining as it has recently been estimated at $0.3 \%$ (Beck et al., 2009). Those born or residing in urban areas are reported to be at greater risk of developing schizophrenia than those born or residing in rural areas (Menezes, 2009). Migrants are additionally thought to be at greater risk of developing schizophrenia (Beck et al., 2009; Menezes, 2009). Whilst the prevalence of schizophrenia in indigenous communities in Australia is unknown, elevated rates of hospitalisation and pervasive 
disadvantage affecting communities suggest that the prevalence may be higher than in the wider community (Australian Institute of Health and Welfare, 2011).

The outcomes for people with schizophrenia are varied and represent a topic of debate amongst researchers. According to Smith et al. (2009), approximately $25 \%$ of consumers will experience just one psychotic episode in their lives and return to a normal level of functioning. Other researchers indicate that whilst variation exists in illness course and outcome amongst consumers, complete remission and return to pre-morbid status is rare (Sharif et al., 2007). It has been reported that about $60 \%$ of consumers will relapse more than once, but will return to pre-morbid levels of functioning in between episodes, whereas the remainder are likely to experience multiple episodes with residual symptoms in between (Birchwood \& Jackson, 2001). Positively, the proportion of consumers whose illnesses improved substantially is thought to have increased significantly after the mid 1950s, coinciding with the introduction of antipsychotic medication (Hegarty et al., 1994).

Current literature, based on a series of investigations conducted by the World Health Organisation, states that the prognosis is better for consumers living in lower and middle income countries compared to those living in wealthy countries, however, conflicting research exists (Menezes, 2009). Poorer outcomes have been associated with insidious onset and delayed initial treatment of the illness, social isolation, a strong family history, living in industrialised countries, male gender and substance misuse (Smith et al., 2009).

Co-morbid substance use amongst people with schizophrenia is widespread (Cuffel, 1996). Estimates of the prevalence of alcohol and drug 
misuse amongst people with schizophrenia vary, but it has been reported as three times higher than that observed in the general population (Green, 2005). It is likely that the true prevalence of substance misuse in this population may be higher than self-report by consumers might indicate (McPhillips et al., 1997). Drugs such as stimulants (e.g. cocaine, amphetamines) increase levels of dopamine in the brain and, thus, tend to worsen the symptoms of schizophrenia (Green, 2005; Mueser \& Gingerich, 2006).

Compared to the general population, schizophrenia is associated with increased mortality. It is estimated that people with schizophrenia have a two to three-fold increased mortality risk compared with the general population, which may have worsened in recent decades (McGrath \& Susser, 2009). There is an increased probability of people with schizophrenia dying prematurely, with suicide posited as a major contributor to this discrepancy (Beck et al., 2009). Approximately 5.6\% of people with schizophrenia die from suicide, which is approximately 13 times greater than the general population and the greatest risk of suicide occurs during the early stages of the illness (Beck et al., 2009). Various studies have also indicated that people with schizophrenia have elevated mortality across a wide array of illnesses (Beck et al., 2009).

Research regarding the prognoses for people with schizophrenia must be treated with caution, however, as factors such as institutionalisation, socialisation into the consumer or patient role, lack of rehabilitation resources, reduced economic opportunities, reduced social status, adverse side effects of medication, lack of staff expectations and loss of hope have been found to impact on, or mimic, the chronicity of the illness (Anthony, 1993; Harding, Zubin \& Strauss, 1992). Additional moderating variables on 
the recovery process that have been proposed include biological and psychosocial therapies, social relationships, cultural determinants, illness behaviour, coping strategies, the consumer's developmental stage, stigma and community attitudes towards mentally ill consumers (McGorry, 1992). A radical perspective in relation to the chronicity of schizophrenia is that what was once thought to have been a result of the illness, is rather, caused by the way consumers are treated by the health system and by society (Anthony, 1993).

\subsection{Stages of recovery}

There are various stages of recovery in schizophrenia which are referred to throughout this thesis. An acute episode, or the active phase, refers to a period of extremely intense psychotic symptoms. It may start suddenly, or develop more gradually over a period of several months (McEvoy et al., 1999). The active phase is usually preceded by a prodromal phase, in which functioning deteriorates and low-grade positive and negative symptoms present (McGorry, 1992). Stabilisation (similar to remission) after an acute episode usually occurs once the intense psychotic symptoms are controlled by medication. During stabilisation there are usually periods of troublesome but much less severe symptoms. The maintenance phase occurs between acute episodes and refers to the longer term recovery phase of the illness. During maintenance, the most intense symptoms of the illness are controlled by medication; however, there may still be some milder, persistent symptoms (McEvoy et al., 1999). Of note, there is considerable variability in the clinical course of schizophrenia, which means that consumers frequently move in and out of recovery (Liberman \& Kopelowicz, 2005). Consumers 
who have achieved stabilisation and maintenance would be expected to relapse, as a result of medication non-adherence, substance use or significant stress, for example.

\subsection{Chapter summary}

The current thesis is concerned with the experiences of people meeting criteria for a diagnosis of schizophrenia. Chronic in nature, schizophrenia is considered one of the most severe and disabling mental illnesses which significantly interferes with functioning in various domains, affecting approximately $0.7-1 \%$ of the population. Schizophrenia symptoms are consistently described by a medical model of clusters of positive, negative and cognitive symptoms. Of significant relevance to the present study, it has been estimated that $60 \%$ of individuals with schizophrenia will experience symptom relapse more than once but will return to premorbid levels of functioning in between episodes. Whilst outcomes for people with schizophrenia are varied, complete remission and return to pre-morbid status is uncommon, with consumers typically experiencing recurrent episodes. Consumers additionally have increased co-morbidities (including high rates of substance misuse) and greater mortality rates compared to the general population, which is partly explained by higher suicide rates and increased mortality rates across a wide range of illnesses. The literature relating to outcomes for people with schizophrenia must be interpreted with caution, however, as several other factors have been proposed to contribute to, or mimic, the chronicity of schizophrenia. The following chapter will discuss treatments for schizophrenia, with a focus on antipsychotic 
medication, which has consistently been shown to significantly improve symptoms and general outcomes for consumers. 


\section{Chapter 2: Treatments for Schizophrenia}

The goal of treatment strategies for schizophrenia is generally to target the acute psychotic phase of the condition and achieve maintenance of the treatment effect with a reduction in the risk of relapse in longer term treatment (Sharif et al., 2007). Medical intervention through antipsychotic medication is currently, consistently regarded as the primary treatment for schizophrenia (Noetzel, Jones \& Conn, 2012). Despite the alarming statistics outlined in the previous chapter, it is important to note that the introduction of antipsychotic medication has significantly improved outcomes for people with schizophrenia.

Whilst antipsychotic medications are of enormous benefit to most people with schizophrenia, they do not represent a cure. Antipsychotic medications usually alleviate symptoms or render them milder and, in some cases, can shorten the course of an episode of schizophrenia. Consumers typically need to trial various antipsychotic medications before they find the regime that works best for them, as well as the optimum dosage (McEvoy et al., 1999). Whilst medication treatment is generally successful in treating positive symptoms, it has been reported that approximately one third of consumers derive little benefit from it and negative symptoms are notoriously difficult to treat (Smith et al., 2009). Although medication is almost always necessary in the treatment of schizophrenia, it has been shown to be more effective when used in conjunction with psychosocial treatments and rehabilitation (McEvoy et al., 1999; Mueser \& Gingerich, 2006;). 
Due to the topic area of the present thesis, the following chapter will focus on medical treatments for schizophrenia, specifically, on antipsychotic medications. This is not to detract from the importance of psychosocial treatments for schizophrenia but rather, reflects an attempt to provide information pertinent to this research. Furthermore, whilst it is acknowledged that other medications are frequently prescribed concurrently to antipsychotic medications to treat schizophrenia, these medications are not discussed in the following section due their vastness and because of the specific focus of this thesis.

The chapter begins with a discussion of the nomenclature used to describe older and newer antipsychotic medications followed by how they are taken and a brief description of how antipsychotics work. The chapter continues to discuss typical and atypical antipsychotic medications, including the results of clinical trials. A summary of current indications for particular routes and medications is then provided. This is followed by a section which highlights the importance of continuous maintenance psychopharmacology to effectively reduce the risk of relapse.

The purpose of this chapter is to highlight the impact that the development of antipsychotic medications has had on the treatment of people with schizophrenia. It also aims to provide the reader with an understanding of the distinction between typical and atypical antipsychotic medications as well as an overview of the mechanism and side effects of typical antipsychotics and atypical antipsychotic medications, which will help to contextualise interview data. Furthermore, the chapter concludes with an emphasis on the importance of early pharmacological intervention and adherence to continuous maintenance medication schedules, which have 
been associated with improved outcomes for people with schizophrenia, thus, reiterating the importance of adherence.

\subsection{Antipsychotic medication nomenclature}

Older antipsychotics are known as 'conventional', 'typical', 'first generation' and 'traditional' antipsychotics (Jones \& Buckley, 2006; Weiden, Scheifler, Diamond \& Ross, 1999). Newer antipsychotics are referred to as 'novel' or 'atypical' antipsychotic medications (Mueser \& Gingerich, 2006). The terms 'typical' and 'atypical' are used in this thesis to distinguish older and newer antipsychotic medications. Whilst there is no consensus regarding the definitions of typical and atypical antipsychotic medications, distinctions between the two are typically described as relating to the propensity to cause extra-pyramidal side effects (EPS) (Sharif et al., 2007). EPS refer to severe neurological side effects, particularly involving dys-regulation of voluntary motor function (Conley, 2000; Weiden et al., 1999). Typical antipsychotic medications are commonly associated with EPS whereas atypical antipsychotic medications have different side effect profiles, operate in different ways and generally, do not cause EPS (Mueser \& Gingerich, 2006).

Whilst the presence or absence of EPS represents the essential difference between typical and atypical antipsychotics, there is a considerable 'grey area' with this distinction, because there is wide intra-group variability, especially in adverse effects profile, between medications (Jones \& Buckley, 2006). For example, high doses of risperidone, an atypical medication, can cause EPS. Furthermore, adverse effects of antipsychotic medications, such as EPS, are predominantly dose dependent and can be influenced by consumer characteristics, including age and gender (Haddad \& Sharma, 
2007). These confounding factors should, thus, be considered in the interpretation of research data comparing medications.

The variation in tolerability of atypical antipsychotic medications and differences between the atypical agents in terms of efficacy and pharmacodynamic profiles also suggest that it is misleading to regard the atypical antipsychotics as a uniform drug class (Haddad \& Sharma, 2007). Nonetheless, experts now strongly recommend atypical antipsychotic medications, as a group (with the exclusion of clozapine), as the first-line of treatment for schizophrenia, replacing typical antipsychotic medications (McEvoy et al., 1999), perhaps solely because of the assumption that they are better tolerated due to a lower propensity to cause EPS.

\subsection{Route of antipsychotic medication}

The most common way of taking antipsychotic medication is in pill or liquid form between one and three times a day. Some antipsychotic medications are also available in tablets, including dissolvable forms (Mueser \& Gingerich, 2006). Antipsychotic medications can additionally be taken in the form of short-acting and long-acting injections.

Short-acting intramuscular formulations are typically used in emergency situations to help people who are acutely psychotic to calm down (Mueser \& Gingerich, 2006). The practice of administering high doses of high potency antipsychotic medication intramuscularly within 24 hours has not demonstrated any gains in the onset of therapeutic efficacy, when compared to the administration of standard doses, however (Sharif et al., 2007). Researchers claim that the practice of administering high doses of 
antipsychotic medication within a short period of time has been largely discontinued due to a lack of evidence (Sharif et al., 2007).

Long-acting, depot formulations, administered intramuscularly, once every one to six weeks are also available for some antipsychotic medications (David \& Adams, 2001). Long-acting injections are frequently prescribed in cases of poor adherence and for consumers on community treatment orders, which permit the involuntary treatment of some consumers whilst they reside in the community. Community treatment orders are typically granted when consumers are non-adherent and experience frequent symptom relapses that place their own or others' safety at risk. Long-acting depot formulations work by distributing medication throughout the body on a constant basis over the following weeks and then gradually leave the body after the one to six weeks have elapsed. Thus, the perceived advantage of this method is that it guarantees consistent delivery of the medication (David \& Adams, 2001).

\subsection{How antipsychotic medications work in brief}

The diagnosis of schizophrenia is associated with demonstrable alterations in brain structure and changes in dopamine neurotransmission, the latter of which is directly related to delusions and hallucinations. Whilst the exact mechanism of antipsychotic medications is unclear, it is frequently proposed that they block dopamine receptors, thereby targeting the positive symptoms of schizophrenia (Van Os \& Kapur, 2009). Consistently, randomised control trials of medication have shown that the potency of different antipsychotic medications can be explained almost perfectly by the amount of dopamine in the brain that is blocked by that medication (Mueser \& Gingerich, 2006). This theory is disputed by research which demonstrates 
that newer (atypical) medications bind less than older (typical) medications to dopamine receptors, yet are as efficacious (or more so in the case of clozapine) (Jones \& Buckley, 2006). Negative symptoms and cognitive symptoms are not consistently improved by antipsychotic medication treatment.

Typical antipsychotic medications block dopamine in the limbic system and additionally reduce dopamine in other areas of the brain, which causes serious EPS (Weiden et al., 1999). Imaging studies of the binding of typical antipsychotic medications to dopamine receptors, as assessed using positron emission tomography (PET), indicate that there is a very narrow dosing range within which one can achieve antipsychotic efficacy without causing EPS. Doses below the dopamine binding threshold (approximately $60 \%$ dopamine receptor occupancy) are clinically ineffective for treating schizophrenia. Doses that increase the dopamine occupancy to $70 \%$ or above will induce EPS (Jones \& Buckley, 2006).

The atypical medications, in general, have a wider therapeutic window, meaning that the difference between therapeutic and toxic levels is larger (Jones \& Buckley, 2006). Atypical antipsychotic medications block dopamine and serotonin receptors. This effect on serotonin is believed to enhance the medication's antipsychotic effect while reducing effects on the parts of the brain that cause EPS (Weiden et al., 1999). Another hypothesis is that the new medications are more selective in the parts of the brain they affect (Weiden et al., 1999). For example, there is more than one type of dopamine receptor, and different antipsychotics may have different effects on each type (Mueser \& Gingerich, 2006). 


\subsection{Typical antipsychotic medications}

The first antipsychotic medication, Chlorpromazine, was released for clinical use on 1954 (Sharif et al., 2007). It was originally termed a 'major tranquiliser' due to its calmative effects in addition to dramatically reducing psychotic symptoms amongst agitated patients (Jones \& Buckley, 2006;

Schulz \& McGorry, 2000; Weiden et al., 1999). The introduction of Chlorpromazine represented the first effective medical management strategy for schizophrenia and was, thus, deemed one of the great medical advances of the twentieth century (Sharif et al., 2007). Typical antipsychotics were breakthrough medications, as they provided therapy for psychosis, which had previously been almost impossible to treat (Conley, 2000). The effectiveness of antipsychotics in reducing the intensity of consumers' positive symptomshas permitted the outpatient treatment of schizophrenia and was associated with a dramatic reduction in mental hospital populations (Freedman, 2005; Schulz \& McGorry, 2000).

The typical antipsychotic medications currently in use include: Haloperidol, Thieridaxine, Thiothixene, Fluphenazine, Trifluoperazine, Chlorpromazine and Perphenazine. Over one hundred clinical trials have demonstrated the effectiveness of typical antipsychotic medications, including a series of double-blind placebo studies (Sharif et al., 2007). Research that has not supported the effectiveness of typical medications is generally restricted to poorly designed studies that involved ineffective dosages (Sharif et al., 2007). Although typical antipsychotic medications substantially reduce the positive symptoms of schizophrenia in some people, they have been documented as having no appreciable effect on cognitive dysfunction, and as having only a limited effect on, and even worsening, negative and depressive symptoms (Conley, 2000; Jones \& Buckley, 2006; 
Mueser \& Gingerich, 2006; Weiden et al., 1999). Contradictory evidence exists, however, which points to clinical trials that indicate that all symptoms associated with schizophrenia improve with typical antipsychotic medication although, in general, positive symptoms respond to a greater degree and more consistently than negative symptoms (Sharif et al., 2007).

Extensive evidence indicates that typical antipsychotic medications are essentially similar in efficacy profiles, however, individual consumers may respond better to one drug than another due to their different side effect profiles (Sharif et al., 2007). For example, while Chlorpromazine is quite sedating, Haloperidol is not (Mueser \& Gingerich, 2006). The side effects of typical antipsychotic medications are frequently distressing and prominent and in addition to sedation, include: slowed thinking, dizziness, sexual dysfunction and sensitivity to sunlight. Anticholinergic side effects are also associated with typical antipsychotics, which include dry mouth, blurry vision, constipation, difficulty urinating and memory problems (Mueser \& Gingerich, 2006).

Perhaps the most well-known, serious side effects associated with typical antipsychotics are EPS, which include acute dystonic reactions (e.g., muscular spasms involving the head and neck, affecting vision), akathisia (including restlessness, agitation, fidgeting, rocking and pacing), Parkinsonism (including stiffness, tremor, shuffling gait and dribbling) and akinesia, also known as 'flat affect'. (Birchwood \& Jackson, 2001; Jones \& Buckley, 2006; Mueser \& Gingerich, 2006).

Tardive dyskinesia is another serious side effect that occurs in approximately $4.5 \%$ of consumers each year for the first five years of treatment and is more common amongst elderly populations (Jones \& Buckley, 2006; Schulz \& McGorry, 2000). Tardive dyskinesia typically 
emerges several months after the commencement of treatment and is potentially a lifelong condition (Weiden et al., 1999). It usually consists of involuntary movements of the head, tongue, lips, hands and feet and can affect speech, posture and sometimes breathing (Birchwood \& Jackson, 2001; Jones \& Buckley, 2006; Mueser \& Gingerich, 2006). Typical presentations of the conditions involve protruding tongue, facial grimaces, and slow rhythmical movements of the hands and feet, sometimes even without the person knowing it (McEvoy et al., 1999). The chances of developing tardive dyskinesia can reportedly be reduced by using the lowest possible effective dose of medication (McEvoy et al., 1999).

Since the development of the new, atypical antipsychotic medications, indications for typical antipsychotic medications are shrinking. McGorry (1992) posits that typical antipsychotic medications, in low dosages, may still have a role amongst a small proportion of consumers, who demonstrated a positive response to typical schedules including remission and good tolerability. They have also been indicated in the acute management of aggression or violence in some patients in the past (McEvoy et al., 1999), however, now clozapine is typically recommended to treat hostility (Buchanan et al., 2010). Advantages of typical antispychotic medications over atypical antipsychotic medications include greater medication familiarity for some consumers and clinicians and they are less expensive (Weiden et al., 1999).

\subsection{Atypical antipsychotic medications}

The introduction of atypical antipsychotic medications prompted a paradigm shift in the pharmacological treatment of schizophrenia. 
Specifically, atypical antipsychotic medications largely replaced the mainstays of treatment for schizophrenia for over 40 years (Conley, 2000). The development of atypical antipsychotic medications has also increased the probability of finding a suitable drug for individuals with schizophrenia, as there are now more options available to consumers and practitioners (Janssen et al., 2006). The wider choice offered by the advent of atypical medications, in addition to their increased tolerability, have been associated with more sustained adherence to prescriptions (Liberman \& Kopelowicz, 2005).

Clozapine, the first atypical antipsychotic medication, manufactured in 1959, was first tested in the 1970 and was discontinued due to serious blood reactions (Weiden et al., 1999). It was reintroduced for use in 1989 in the United States, followed by the introduction of the other atypical antipsyhotic medications in the 1990 s or after 2000 (Weiden et al., 1999). The atypical antipsychotic medications currently available are: clozapine, risperidone, olanzapine, quetiapine, ziprasidone, aripiprazole, sertindole, zotepine and amisulpiride. With the exception of clozapine, these atypical antipsychotic medications were developed following years of scientific research aimed at understanding how typical antipsychotic medications work and trying to make more effective medications with fewer serious side effects (Mueser \& Gingerich, 2006).

Several studies have historically supported, and continue to support, the efficacy of atypical antipsychotic medications for treating positive symptoms of schizophrenia amongst treatment-responsive, recurrentepisode consumers, when compared with placebo (eg; Buchanan et al., 2010). A recent systematic review revealed some evidence that olanzapine, 
ziprasidone and zotepine were more effective at reducing relapse rates over 12 months than placebo (Smith et al., 2009).

A vast number of double-blind studies comparing the acute treatment effects of atypical antipsychotic medications with typical antipsychotic medications have been conducted. Many studies have claimed advantages of atypical medications over typical medications in terms of effectiveness. For example, it is often stated that atypical antipsychotics are more effective at treating negative symptoms and cognitive impairment and frequently lead to a better quality of life for consumers (Meltzer, 1999; Mueser \& Gingerich, 2006). Furthermore, many experienced clinicians reportedly believe that the atypical medications are even better for negative symptoms than controlled trials have shown, as they have an antidepressant effect (Weiden et al., 1999). They have also been associated with cognitive-enhancing effects (Lewis \& Lieberman, 2008). Research additionally indicates that atypical antipsychotics are highly tolerable, particularly for individuals with affective disorders and substance abuse problems, the young and elderly consumers (Conley, 2000). However, it is difficult to interpret the true advantages of atypical medications over typical medications in terms of efficacy, despite a significant body of research that indicates so. For example, data used to support the effectiveness of atypical antipsychotic medications over typical antipsychotic medications in treating negative symptoms is difficult to interpret because EPS that are commonly associated with typical medications are almost impossible to distinguish from the negative symptoms of schizophrenia (Sharif et al., 2007).

Atypical antipsychotic medications have a lower propensity of atypical medications to cause EPS, even at the high end of therapeutic dosage (Meltzer, 1999). Furthermore, they have been reported to be up to ten times 
less likely to induce tardive dyskinesia than typical antipsychotic medications (Jones \& Buckley, 2006). Of note, clozapine is associated with virtually no risk of tardive dyskinesia, and has even been shown to reduce symptoms of the condition (Jones \& Buckley, 2006; Weiden et al., 1999). Although anticholinergic side effects can still occur with some atypical antipsychotic medications, they are reportedly usually less severe than those associated with typical antipsychotic medications (Mueser \& Gingerich, 2006).

Atypical antipsychotic medications are associated with other serious side effects, however. In particular, they are more likely to cause metabolic effects, including the propensity to cause more weight gain and glucose elevation when compared with typical medications. Metabolic effects additionally increase consumers' risk of developing diabetes, lipid abnormalities and coronary artery disease (Conley, 2000; Mueser \& Gingerich, 2006). Like the typical antipsychotic medications, there is a considerable amount of intra-group variation of side effects amongst the atypical group of medications.

In addition to research focused on effectiveness in treating symptoms and side effects, the schizophrenia literature also compares atypical antipsychotic medications with typical antipsychotic medications in terms of adherence rates, relapse likelihood, economic impact and amongst different populations of consumers. For example, research suggests that individuals who switch from typical antipsychotic medications to atypical antipsychotic medications are more adherent than those who continue taking typical antipsychotic medications (Janssen et al., 2006). Studies have also frequently associated atypical antipsychotic medications with reduced relapse rates compared to typical medications and report lower rehospitalisation rates with atypical medications compared to typical antipsychotic 
medications (Conley, 2000; Weiden et al., 1999). In their systematic review and meta-analysis of studies assessing the potential of atypical medications to decrease relapse rates in schizophrenia, Leucht et al. (2003) demonstrated that atypical antipsychotic medications are modestly but significantly better at preventing relapse than typical antipsychotic medications amongst people with schizophrenia. Pharmaco-economic studies indicate that atypical antipsychotic medications are more economical than the typical antipsychotic medications, despite their disproportionately higher prescription costs, which is attributed to lower hospitalisation rates associated with atypical antipsychotic medications (Jones \& Buckley, 2006). Atypical antipsychotic medication, clozapine, has been demonstrated to be effective in treating treatment-resistant schizophrenia (Buchanan et al; 2010; McGorry, 1992). Treatment resistance is failure of full remission of positive symptoms or the lack of satisfactory clinical improvement despite sequential use of recommended doses of two or more antipsychotic medications for six to eight weeks (McGorry, 1992). Recent studies also continue to support the efficacy of clozapine for persistent aggressive and hostile behaviours in people with schizophrenia, including those who do not meet criteria for treatment resistant schizophrenia (Buchanan et al., 2010). There has been limited research conducted on the effectiveness of other atypical antipsychotic medications and typical medications for the treatment of hostility, however.

\subsection{Contradictory evidence}

The Clinical Antipsychotic Trials of Intervention Effectiveness (CATIE) were part of a multi-site, double-blind study which was funded by the National Institute of Mental Health (United States), conducted between 
2001 and 2004. They represent one of the largest, non-industry sponsored comparisons of typical and atypical antipsychotic medications in people with multi-episode schizophrenia, designed to mimic clinical practice (Buchanan et al., 2010). CATIE compared the long-term effectiveness of various antipsychotic medications, including perphenazine, a typical antipsychotic medication, and other atypical antipsychotic medications: olanzapine, risperidone, quetiapine and ziprasidone (Freedman, 2005). One of the aims of the CATIE trials was to investigate the relative effectiveness of atypical and typical antipsychotic medications which investigators described as being “incompletely addressed" by previous research (Lieberman et al., 2005).

Time to discontinuation of medication for any reason was the primary outcome variable assessed in the CATIE. Secondary outcomes assessed were discontinuation due to: lack of efficacy, tolerability, patient decision, physician decision, psychopathology, safety, neuro-cognitive functioning and cost-effectiveness (Sharif et al., 2007). Symptoms were assessed, as measured by Positive and Negative Symptoms Scale (PANSS) scores. Participants were randomly assigned to different medication groups and dosages ranged. If the assigned treatment was discontinued for any reason, participants were given the option of switching to another treatment group. The results of the CATIE were discouraging and, to a degree, dispute assertions of the superior efficacy of atypical antipsychotic medications compared to typical antipsychotic medications. No drug in the trial, including the atypical medications, provided the majority of participants with treatment that lasted for the full 18 months of the study (Freedman, 2005; Lieberman et al., 2005). The overall average rate of discontinuation for any reason for all the medications involved in the trials was $74 \%$ (Lieberman et al., 2005). These results indicate that treating schizophrenia, even with the 
newer, atypical antipsychotic medications, is only partially effective (Freedman, 2005). Although olanzapine had the lowest discontinuation rate of all medications involved in the study, this was only statistically superior to risperidone and quetiapine and not to ziprasidone or perphenazine (Lieberman et al., 2005; Sharif et al., 2007). Additionally, whilst analysis of secondary outcome measures yielded that time for discontinuation due to lack of efficacy was longer in the olanzapine group compared to all other groups, olanzapine had the highest rate of discontinuation due to side effects (18\%) and was associated with metabolic side effects in particular (Sharif et al.,; 2007).

The second phase of the CATIE trials involved the large number of participants who discontinued their assigned medication in the first trials. These participants were offered entry into one of two treatment branches. If they discontinued the first medication due to lack of efficacy, they were offered entry into a randomised control trial comparing open-label clozapine with another atypical (blinded treatment) not previously administered in the previous phase (olanzapine, risperidone or quetiapine). Again, the primary outcome measure in this trial was time until discontinuation for any reason. Overall, for those who had previously failed to improve with an atypical medication, clozapine was more effective than switching to another atypical medication. These data thereby support the use of clozapine for people with treatment-resistant schizophrenia.

The UK Cost Utility of the Latest Antipsychotic Drugs in Schizophrenia Study (CUtLASS) represents another large, non-commercial research project, which compared typical and atypical medications in consumers with multiepisode schizophrenia (Buchanan et al., 2010). CUtLASS comprised a pair of smaller, open randomised trials comparing typical and atypical medications 
(except for clozapine) and other atypical medications with clozapine (Lewis \& Lieberman, 2008). Results of the CUtLASS were consistent with those from the CATIE. Specifically, it too revealed that atypical medications were no more effective or better tolerated than typical medications and clozapine outperformed other atypical medications in terms of efficacy (Lewis \& Lieberman, 2008). Recent systematic reviews provide further support for these results. Smith et al. (2009), for example, reported moderate evidence that antipsychotic medications were equally as effective as each other at preventing relapse rates, which included studies comparing typical and atypical medications.

The inconsistency of results from CATIE and CUtLASS, two nonindustry funded trials, with published results of "head-to-head" trials comparing the efficacy of atypical antipsychotic medications, which are mainly funded by pharmaceutical companies, suggests that antipsychotic medication research results must be interpreted with caution. Indeed, it is claimed that much of the evidence about the relative efficacy of atypical medications over typical medications stems from short-term, industryfunded trials where participants are highly selected, drop-out rates are high and outcomes are based mainly on statistical comparisons of symptom ratings (Lewis \& Lieberman, 2008). Controversially, Heres et al. (2006) reviewed the results of "head-to-head" studies of atypical antipsychotics from 1963 to 2003 and found that a relationship existed between the sponsor of the trial and the drug favoured in the study's overall outcome. Not surprisingly, in $90 \%$ of the studies the overall outcome was in support of the sponsor's drug (Heres et al., 2006). These findings indicate that conclusions about the efficacy of atypical antipsychotic medications remain contradictory, especially when findings of studies of the same drugs but different sponsors 
are compared. It is acknowledged that this thesis may report some potentially biased results of medication trials.

\subsection{Summary of indications for typical and atypical medications, different routes of administration and dosages}

Whilst McGorry (1992) recommends that oral atypical medications risperidone, olanzapine, quetiapine, amisulpride and aripiprazole - are prescribed as first and second line treatments in first episode psychosis, more recent research recommends that acute positive symptoms in first episode schizophrenia are treated with any antipsychotic medications, with the exception of clozapine and olanzapine (Buchanan et al., 2010). Buchanan et al. (2010) point out that several studies suggest no significant short-term efficacy differences between typical and atypical medications and rule out clozapine and olanzapine due to their higher propensities for side effects.

It is recommended that consumers on typical antipsychotic schedules, who are experiencing a poor response (indicated by persistent positive or negative symptoms) and/or distressing side effects, should be switched to oral, atypical medications (McGorry, 1992). Due to criticisms about side effects, clozapine is currently only the treatment of choice for individuals who have failed to adequately respond to several antipsychotic trials, have high levels of suicidality or high levels of hostility (Buchanan et al., 2010). McGorry (2004) stresses the importance of introducing clozapine at the earliest convenience in the instance of evidence of treatment-resistance to achieve best outcomes amongst this group of consumers.

Long-acting depot medication is implicated for consumers who voluntarily elect to receive medication via this route. It is also indicated for 
those who experience significant, ongoing adherence difficulties, where the consequences are relapse or significant risk to themselves or others (McGorry, 1992). Consumers who are on community treatment orders typically take long-acting, depot medications.

The time to onset of therapeutic effect of antipsychotic medications during the treatment of an acute episode of schizophrenia is variable (Mueser \& Gingerich, 2006; Sharif et al., 2007). Antipsychotic medications typically produce noticeable changes within one and three weeks however most gains in effectiveness are noted within six to eight weeks of administration (and about 12 weeks for clozapine) (McEvoy et al., 1999; Sharif et al; 2007). It is noteworthy, however, that as many as approximately one third of consumers fail to respond to antipsychotic medication (Smith et al., 2009). Research consistently indicates that initial dosage of antipsychotic medication should be low (Buchanan et al., 2010; McGorry, 1992), because people with firstepisode acute episodes of psychosis tend to exhibit increased medication responsiveness and experience increased sensitivity to side effects. In general, because the incidence of side effects increases with the use of doses at the higher end of the recommended range, the lowest effective use should be used for maintenance treatment (Buchanan et al., 2010). As individuals with schizophrenia often experience symptoms of anxiety, depression, and hostility, which are not amenable to antipsychotic treatment, adjunctive treatment with benzodiazepines is frequently used to treat these ancillary symptoms (Buchanan et al., 2010). 


\subsection{Continuous maintenance pharmacotherapy}

The continued use of antipsychotic medication, or medication maintenance, following acute treatment is well established because of the high rate of relapse associated with medication discontinuation. As previously noted, consumers' positive symptoms can typically be stabilised within six weeks from the time they start medication. Once symptoms have been effectively reduced, continuing to take antipsychotic medications on a regular basis can help to stabilise the illness more generally (Mueser \& Gingerich, 2006). Indeed, it has been estimated that after recovering from a relapse, a person on a continuous maintenance schedule of typical antipsychotic medication has a $45 \%$ less chance of having a second relapse within a year compared to those on no medication (Weiden et al., 1999).

Early detection and initiation of maintenance antipsychotic treatment for people with schizophrenia is also important. A study into early psychosis reported that duration of untreated psychosis is a modest independent predictor of outcome (Schimmelmann et al., 2008). Specifically, a longer duration of untreated psychosis was associated with worse premorbid functioning and outcomes deteriorated with increments of delay in treatment. Additionally, Haas, Garrett and Sweeney (1998) reported that a delay of one or more years between onset of symptoms and initiation of antipsychotic medication was related to more severe negative symptoms at admission and more positive and negative symptoms at discharge. It has been found that people experiencing first-episode psychosis often do not initiate help-seeking, particularly if they have a family history of mental illness (O’Callaghan et al., 2010).

While the optimal duration of maintenance treatment in a remitted first-episode case of schizophrenia remains unknown, treatment guidelines 
generally recommend at least one year of antipsychotic treatment and some consider indefinite maintenance treatment reasonable (Perkins et al., 2006). The results of a longitudinal study which monitored a group of first episode consumers supported continuation of maintenance medication treatment for at least two years after the initial episode and provided support for the continued importance of maintenance medication beyond this time (Robinson et al., 1999). According to a survey of experienced clinicians, the recommended duration of maintenance antipsychotic medication therapy varies depending on the severity of schizophrenia. First episode consumers who have gone into remission after the acute episode has resolved are recommended to take medication for 12 to 24 months. When a diagnosis of schizophrenia is clearly established by multiple episodes and/or persistent symptoms, longer term or lifetime medication is recommended. For elective dose reductions, it is recommended that medication is tapered gradually at two to four week intervals over a period of several months rather than switching abruptly to the targeted lower dose (McEvoy et al., 1999).

Dose reduction strategies have been trialled as alternatives to continuous maintenance schedules in several studies, representing attempts to overcome the adverse side effects of antipsychotic medications whilst still treating the symptoms of schizophrenia. Schooler (2004) reviewed research involving two dose reduction strategies; continuous low dose and intermittent or targeted medication. Although the continuous low dose strategy was associated with reduced adverse side effects and improved subjective well-being for consumers, maintenance medication on moderate dose regimes were consistently found to be the most effective in preventing relapse and, thus, were considered to have largely better outcomes for consumers. Targeted or intermittent medication did not prevent relapse and 
did not exhibit any clear benefits in terms of reducing adverse side effects (Schooler, 2004). Consistently, in a more recent review, Buchanan et al. (2010) reported an increased risk of symptom worsening and relapse associated with targeted, intermittent strategies compared to continuous maintenance strategies. It is further suggested that side effects such as tardive dyskinesia are more common in people who are intermittent in their medication-taking patterns and that sub-optimal antipsychotic treatment can potentially result in the emergence of disabling, treatment-resistant symptoms (Perkins et al., 2006). Intermittent approaches are, therefore, not recommended unless the consumer refuses continuous medication treatment (McEvoy et al., 1999).

\subsection{Chapter summary}

The present chapter has focused the treatment of schizophrenia with typical and atypical antipsychotic medications. The interviewees in the present research were all asked to discuss their experiences of taking typical and/or atypical medications, thus, it is hoped that this chapter helps to contextualise interview data. The introduction of antipsychotic medications revolutionised the treatment of people with schizophrenia. Antipsychotic medications are currently available in tablet and liquid forms and short and long-acting intramuscular depot formulations. Whilst the exact mechanism of antipsychotic medications is unclear, it is often proposed that they block dopamine receptors in the brain, thereby targeting the positive symptoms of schizophrenia. The older and newer antipsychotic medications have been distinguished as "typical" and "atypical" medications, primarily on the basis of their propensity to cause EPS. Whilst typical antipsychotic medications 
are still used, they have largely been replaced by atypical medications as the first-line treatment of schizophrenia due to their reported increased efficacy, tolerability and because they have been associated with a lower risk of relapse when compared to typical medications. Such findings have been disputed by recent reviews and large-scale, non-industry funded studies - the CATIE and the CUtLASS - which both reported no significant differences between typical and atypical medications in their effectiveness in treating symptoms and preventing relapse.

Thus, there are some inconsistencies in relation to guidelines for indications of typical and atypical medications, in particular, whether atypical medications or both typical antipsychotic medications and atypical antipsychotic medications, should represent the first-line treatment for first episode consumers. Clozapine is the medication of choice for treatment resistant schizophrenia. Long-acting depot medication is recommended when consumers express a preference for this route and for those experiencing significant adherence difficulties. It typically takes approximately six weeks for the onset of the therapeutic effects of antipsychotic medication. Early initiation of medication treatment amongst first episode consumers has been associated with better outcomes for consumers. Continuous maintenance pharmacotherapy is superior to dose reduction strategies and intermittent, targeted medication regimens in preventing relapse. The benefits associated with continuous maintenance pharmacotherapy support the importance of complete adherence (as opposed to partial adherence) in order to prevent relapse, thus, reinforcing the benefits of research that explores adherence amongst consumers. The following chapter will elaborate the importance of medication adherence 
amongst consumers, in addition to providing an overview of adherence statistics and factors proposed to influence adherence. 


\section{Chapter 3: Medication Adherence}

Previous chapters have established that typical and atypical medication treatment is effective in treating the positive symptoms of schizophrenia. Moreover, a continuous maintenance medication schedule can reduce the risk of relapse amongst consumers and is significantly more effective than dose reduction or intermittent strategies. Positive outcomes in terms of symptom reduction and reduced risk of relapse are contingent upon consumers' adherence to continuous maintenance medication schedules, however. In contrast, non-adherence has been shown to be the most important predictor of relapse and hospitalisation amongst consumers. Despite these negative consequences, rates of non-adherence remain high amongst consumers.

Following a brief account of the terminology used to describe the behaviour of medication taking, the following chapter summarises research related to the impact of adherence on symptoms and relapse. Statistics that relate to the prevalence of adherence are then provided, however, they should be interpreted with caution due to the difficulties associated with measuring adherence accurately. This is followed by a discussion of factors proposed to influence adherence in qualitative and quantitative research. An overview of the Health Belief Model, which has been proposed to explain adherence behaviour amongst consumers with schizophrenia, is then presented. By highlighting the benefits associated with adherence for consumers and providing statistics which illustrate how common non-adherence is, the present chapter supports the value of research aimed at improving adherence amongst consumers. Furthermore, the summary of quantitative and 
qualitative research exploring factors related to adherence, in addition to explanatory models of adherence, provide a comprehensive overview of previous findings. Indeed, there is some overlap with previous findings in the analysis presented in subsequent Chapters 5, 6 and 7.

\subsection{Terminology}

Various terms have been used to describe the behaviour of continuing to take medication as prescribed. The most commonly used, traditional term is compliance, which has been defined as the extent to which a consumer's behaviour matches the prescriber's recommendations (Horne, Weinman, Barber, Elliot, \& Morgan., 2005). The use of the term compliance is declining as it implies a lack of consumer involvement and, rather, suggests a passive approach whereby the consumer faithfully (and often unquestioningly) follows the advice and directions of the healthcare provider (Horne et al., 2005; Swaminath, 2007). Inherent to the various definitions of compliance is the assumption that medical advice is good for the consumer and that rational consumer behaviour means following medical advice precisely (Swaminath, 2007).

The term adherence has been adopted by many as an alternative to compliance. Adherence is defined as the extent to which the consumer's behaviour matches agreed recommendations from the prescriber (Horne et al., 2005). It reduces attribution of greater power to the healthcare provider in the prescriber-consumer relationship and, rather, denotes some collaboration regarding health-related decisions (Swaminath, 2007). Adherence represents an attempt to emphasise that a consumer is free to decide whether to adhere to the health provider's recommendations and that 
failure to do so should not be a reason to blame the patient (Horne et al., 2005). According to Swaminath (2007), utilising this terminology with the consumer assists in fostering ownership and the continuation of treatment decisions by the consumer.

Another new term which is predominantly used in the United Kingdom is concordance. The definition of concordance focuses on the consultation process, in which healthcare provider and consumer agree to therapeutic decisions that incorporate their respective views (Horne et al., 2005). The term 'persistence' has also been used recently and refers to the act of continuing treatment for the prescribed duration, or alternatively, the duration of time from initiation to discontinuation of therapy (Cramer, 2008).

Despite some changes throughout the course of the present research, the term adherence was ultimately used, in line with the increased focus on consumer-centred approaches in healthcare. Interview data which will be discussed in the analysis in greater depth (in particular Chapter 7), however, suggest that the term adherence may not accurately reflect current clinical practice. That is, whilst the term adherence implies increased collaboration between the healthcare provider and the consumer, and suggests that consumers have the freedom to choose whether or not to follow a prescribed treatment regimen, in practice, many consumers perceived a lack of control over their treatment regimens. Indeed, many of the individuals with schizophrenia who were interviewed had not previously heard of the term 'adherence' but understood the term 'compliance' and used this to describe the degree to which they followed their medication prescriptions. 


\subsection{Impact of adherence on symptoms and relapse}

Research has consistently shown that medication adherence amongst people with schizophrenia is associated with better outcomes in terms of symptom severity, relapse and general functioning, when compared to nonadherence. Several studies have shown that illness symptoms are more pronounced amongst individuals with schizophrenia who are non-adherent. Extreme exacerbations in symptoms often lead to a relapse of psychosis for non-adherent consumers and hospitalisation.

A recent study, which followed up outpatients with schizophrenia over three years found that symptom remission was more likely to occur in consumers who were adherent to their medication at follow-up (Novick et al., 2010). By contrast, Rosa, Marcolin and Elkis (2005) found that nonadherent consumers presented with an initial worsening of symptoms, which remained constant over one year follow-up. Furthermore, in their study comparing symptom severity amongst consumers who were hospitalised, Janssen et al. (2006) found that the absolute difference in Positive and Negative Syndrome Scale (PANSS) scores measured at admission and at discharge was significantly greater for patients who were more adherent. The higher absolute and relative reduction of PANSS ratings for patients with better adherence was also significant for all sub-scores, including the positive, negative, general psychopathology, and schizophrenia sub-score (Janssen et al., 2006). Non-adherence has also been associated with an increased risk of violence, outpatient treatment program dropout, housing instability and homelessness compared with adherence to treatment programs (Compton, 2007; Olfson et al., 2000). Novick et al. (2010) additionally found significantly higher rates of attempted suicide amongst outpatients who were non-adherent at follow-up. 
Medication adherence has consistently been shown to reduce the high rates of relapse amongst people with schizophrenia (i.e., Lacro, Dunn, Dolder, Leckband \& Jeste, 2002; Novick et al., 2010). It has recently been estimated that $75 \%$ of people with schizophrenia will experience relapses and ongoing associated disability (Smith et al., 2007) and the risk of relapse is reportedly particularly high in the first five years after the first episode (Robinson et al., 1999). Each month after discharge from hospital, approximately 3.5\% of consumers experience symptom relapse (Lader, 1998). Leff and Wing (1971) conducted a landmark study whereby outpatients with schizophrenia were prescribed a low daily dose of oral, typical antipsychotic medication in a double-blind trial, which was shown to lead to a 50\% reduction in the risk of relapse within one year of the acute episode. According to Valenstein et al. (2004), 50-75\% of consumers will relapse within a year if antipsychotic medications are discontinued compared to $25 \%$ of patients who continue their medications.

In a review of relevant literature on adherence, Fenton, Blyler and Heinssen (1997) reported an unequivocal link between non-adherence and relapse and hospitalisation, citing seven studies which indicated that consumers rated as non-adherent have a six month to two year risk of relapse that is an average of 3.7 times greater than consumers rated as adherent. The magnitude of elevated risk of relapse associated with non-adherence was comparable to that reported for randomisation to placebo groups in maintenance antipsychotic medication trials (Fenton et al., 1997). More recently, in their longitudinal study involving first episode consumers with schizophrenia and schizoaffective disorder, Robinson et al. (1999) reported that discontinuing antipsychotic medication therapy increased the risk of relapse by almost five times. It was additionally found that consumers who 
discontinued medication twelve months after the first episode had high rates of second and third relapses, despite careful monitoring by a dedicated research treatment team. In summary, non-adherence to antipsychotic medications following discharge from an acute hospitalisation has been described as the single, most significant risk factor for relapse (Compton, 2007).

Whilst antipsychotic medication therapy does not prevent symptom relapses, it can extend the intervals between relapses and render psychotic episodes less severe. There is some evidence to suggest that the longer a patient survives without relapse, the lower the risk of relapse becomes, thus, highlighting the long-term benefits associated with adherence (Birchwood \& Jackson, 2001). Additionally, if a relapse does occur, despite the beneficial effects of taking medication, it reportedly tends to be milder and can usually be effectively treated by temporarily increasing the dosage of antipsychotic medication (Mueser \& Gingerich, 2006). The prevention of multiple relapses and extension of periods between episodes is critical, as not only are relapses distressing, causing significant emotional and psychological damage, but they can also lead to irreversible social consequences and increase the likelihood of symptoms persisting between episodes (Birchwood \& Jackson, 2001)

Non-adherence amongst people with schizophrenia has also been shown to be significantly associated with increased rate and length of rehospitalisation, increased costs of care as well as increased risk of emergency room visits (Compton, 2007; Lacro et al., 2002; Novick et al., 2010; Olfson et al., 2000). In their study which examined the medical histories of 63 consumers who were regularly hospitalised, Weiden and Glazer (1997) reported that the most common reason for hospitalisation was non-adherence, which accounted for 50\% of cases. Moreover, with each 
missed day of medication, the risk of rehospitalisation increases.

Rehospitalisation is reportedly four times higher among patients who miss 30 days or more over a year compared to those who take their medication as prescribed (Kozma \& Grogg, 2003 cited in Masand \& Narasimhan, 2006). Regarding the costs associated with non-adherence, Masand and Narasimhan (2006) point out that not only are the direct, medical costs high, particularly when there are recurrent hospitalisations and ongoing outpatient care is required, but the indirect costs related to disability and unemployment are even greater.

Even partial non-adherence can lead to worsening symptoms and an increased risk of relapse and rehospitalisation. In their review of the literature, Fenton et al. (1997) reported a significant association between irregular medication adherence and relapse (although there were contradictory findings regarding the relationship between number of hospital admissions and adherence). Irregular adherence has been associated with approximately a two-fold increase in hospitalisation and a four-fold increase in number of days hospitalised in another study, however (Svarstad, Shireman \& Sweeney, 2001). Such data provide further support for the importance of adherence to continuous, maintenance medication schedules.

\subsection{Rates of non-adherence}

Research indicates that among consumers with psychiatric disorders, approximately one third take their medication as prescribed; one third take their medication erratically by either taking lower doses or missing doses; and one third do not take their medication at all (Marder, 1998). Amongst people with schizophrenia, more specifically, the prevalence of non- 
adherence with maintenance antipsychotic medications has been reported in the range of $30-50 \%$ for oral and $10-15 \%$ for depot preparations in early studies (Johnson, 1993; Kane \& Borstein, 1985). A more recent French study reported that $30 \%$ of inpatients were considered to be non-adherent (Dassa et al., 2010), similar to the $28.8 \%$ rate of non-adherence observed in a study which borrowed data from a three year, European, prospective, observational study (Novick et al., 2010). Such figures may be optimistic, however, in the context of several recent studies which have reported non-adherence rates between 40 and 55\% (Compton, 2007; Gray, Wykes \& Gournay, 2002; Lacro et al., 2002; Rosa, Marcolin \& Elkis, 2005; Yang et al., 2011).

Moreover, the results of CATIE (see Chapter 2 for an extended discussion), which monitored consumers on typical and atypical medications over an 18 month period, revealed that approximately $74 \%$ of consumers discontinued their medication (Lieberman et al; 2005). Further complicating the measurement of adherence amongst people with schizophrenia is the fact that rates have been demonstrated to change over time. For example, Weiden et al. (1994) quote non-adherence figures amongst discharged schizophrenia consumers as at least 50\% after one year of treatment and $75 \%$ after two. In an attempt to overcome potential social desirability bias amongst consumers' self-reports of adherence, Moritz et al. (2009) conducted an anonymous survey study, which revealed that $71 \%$ of participants were non-adherent at some stage in their lives. A recent review of the literature relating to adherence amongst people with schizophrenia suggested that if a high standard of ideal adherence is applied, less than $25 \%$ of consumers will meet the criterion (Mitchell, 2007).

It is frequently stated that adherence in psychiatric populations is no better or worse than in other medical settings (e.g., Rogers et al., 1998). 
According to Mitchell (2007), adherence with antipsychotic medication is at least as poor as adherence with medication for physical health problems. Following a review of previous studies of treatment adherence, Cramer and Rosenheck (1998) concluded that an average of $58 \%$ of patients receiving antipsychotics were adherent with medication compared to $76 \%$ of patients prescribed medication for physical disorders, with inter-individual variation in adherence behaviour evident for both groups. In order to explain the high rates of non-adherence amongst people with schizophrenia and people with chronic physical illnesses alike, Baldessarini (1994) proposed that the acceptance of prolonged medical treatment may be fundamentally inconsistent with human nature.

True rates of non-adherence are difficult to quantify in schizophrenia for methodological reasons, thus, accounting for the significant variation in rates reported. Some of the ways that adherence amongst people with schizophrenia has been measured include reliance on patient or relative selfreport, prescription renewals, pill counts, using electronic adherence monitors on pill bottle caps, saliva and urine screens and steady-state serum determinations (Battaglia, 2001; Fenton et al., 1997). None of these methods provide a completely reliable indication of adherence, however (Masand \& Narasimhan, 2006). Statistically evaluating medication adherence is additionally complicated by varying definitions of adherence. As Battaglia (2001) points out, medication adherence is rarely an all-or-none phenomenon as it can include errors of omission, mistakes in dosage and time taken as well as taking medications not currently prescribed. Variations in clinical setting, study duration and characteristics of the study population may further account for differences in the reported prevalence of adherence (Mortiz et al., 2009; Novick et al., 2010). Weiden et al. (1994) state that a 
major problem with studies which adopt a quantitative approach to adherence is that they frequently measure only adherence and fail to elucidate the underlying motivations and attitudes associated with adherence behaviours, which are integral to the management of non-adherence.

\subsection{Factors proposed to influence adherence}

A major challenge in the mental health field, as with other areas interested in adherence behaviour, has been to understand the reasons why consumers may or may not take their medication. Thus, several attempts have been made to assess which specific factors influence adherence to antipsychotic medications amongst people with schizophrenia, with varying results. Studies that have investigated adherence to medications amongst mental health consumers vary in their definitions of adherence, methodology used and study populations, rendering interpretation of results across studies difficult and accounting for differences in findings (Julius, Novitsky \& Dubin, 2009). The following section summarises a selection of quantitative research on predictors of medication adherence. A summary of the extant qualitative research related to adherence amongst people with schizophrenia is then presented. Of note, there has been significantly less qualitative research conducted in the topic area. Due to the limited amount of qualitative research directly related to adherence to medication amongst people with schizophrenia, results from some studies that explored adherence amongst psychiatric populations and chronically ill consumers more generally have also been summarised. 


\subsubsection{The Rating of Medication Influences Scale}

The Rating Of Medication Influences Scale (ROMI) is a questionnaire designed to measure the extent to which various factors influence adherence amongst people with schizophrenia (Weiden et al., 1994). Devised in 1994 and updated in 1995 (Barnes, Andrews \& Awad, 2000), development of the ROMI involved the collation and analysis of results of early studies of adherence. It was developed as part of a longitudinal study of antipsychotic non-adherence and was administered to 115 outpatients with schizophrenia. Items of the ROMI were devised following a review of the non-adherence literature in medical and psychiatric populations, group discussions and testing of preliminary questions with consumers whose attitudes towards medication and adherence behaviours were known (Weiden et al., 1994).

The general adherence domains relevant to schizophrenia and assessed in the ROMI are disease features, treatment system features, therapeutic regimen features, patient-therapist interactions, patient characteristics, psychosocial factors and psychological factors (Weiden et al., 1994). Structured items of the ROMI are divided into two sections, the former measuring adherence-related items (1-7) and the latter measuring non-adherence-related items (8-20). In the first part of the ROMI, items assess the degree of influence on adherence of perceived daily benefit of medication, positive relationship with clinician, positive relationship with therapist, positive family belief, relapse prevention, pressure/force and fear of rehospitalisation. Part two contains items pertaining to no perceived daily benefit, negative relationship with clinician, negative relationship with therapist, practitioner opposed to medication, family/friend opposed to medication, access to treatment problems, embarrassment or stigma over medication or illness, financial obstacles, substance abuse, denial of illness, 
medication currently unnecessary, distressed by side effects and desires rehospitalisation (Weiden et al., 1994). Studies using scales that measure subjective responses to antipsychotic medications such as the ROMI have demonstrated a relationship between negative subjective response to antipsychotic treatment and non-adherence (Barnes et al., 2000). Furthermore, the ROMI is considered the most comprehensive measure for rating adherence, particularly in outpatient settings (Rosa, Marcolin \& Elkis, 2005).

\subsubsection{Literature reviews}

Fenton et al. (1997) conducted a substantive literature review to elucidate factors that influence adherence. They found no consistent associations between demographic factors and adherence. In relation to illness characteristics, the studies reviewed yielded no relationship between adherence and age at onset and duration of illness, age at first hospitalisation and premorbid functioning. Of the eight studies reviewed which assessed the relationship between illness symptom severity or global functioning and inpatient medication refusal or future outpatient non-adherence, one reported an association between more severe psychopathology including disorganisation, hostility and suspiciousness and inpatient drug refusal and five studies linked symptom severity at or after discharge to poor outpatient adherence or poor attitudes towards medication. One study also linked the grandiosity score on the Brief Psychiatric Rating Scale to poor adherence. Whilst the authors did not find support for an association between memory or cognition on adherence, they acknowledged that a significant percentage of outpatients attributed non-adherence to forgetting or indicated that 
reminders to take their medication would be of assistance. Poor insight, as measured by a variety of self-report instruments assessing illness awareness, was consistently linked with non-adherence. Three studies showed an association between poor insight at admission or during hospitalisation and non-adherence in inpatient settings. Four studies linked lack of insight at admission, discharge or post-discharge assessment to poor outpatient adherence. Fenton et al. (1997) acknowledged that although the statistical relationship between insight and adherence has been replicated in a variety of settings, several investigators noticed sizable subgroups of consumers who did not believe they were ill or required medication but were, nonetheless, regularly adherent.

Consistent with Fenton et al.'s (1997) findings, in a 2002 literature review, Lacro et al. found no consistent associations between demographic factors, including age, gender, marital status and education level and adherence. Poor insight, negative attitude or subjective response to medication, substance abuse, shorter illness duration, inadequate hospital discharge planning and poor therapeutic alliance were the risk factors found to be most consistently associated with non-adherence. There was an absence of support for relationships between illness-related factors, including neuro-cognitive impairment, severity of positive symptoms and the presence of mood symptoms and adherence. Furthermore, the severity of medication side effects, dose of medication, route of medication administration and family involvement were not found to be consistent predictors of nonadherence. However, a limitation of the review was that many of the studies included were retrospective, cross-sectional and conducted prior to the introduction of atypical antipsychotic medications. Given Fenton et al.'s 
literature review was conducted in 1997, this too likely precluded the inclusion of several atypical medications.

More recently, Compton (2007) reviewed relevant literature and developed a predictive model of risk factors for non-adherence to antipsychotic medications and follow-up appointments amongst people with schizophrenia. The model is comprised of eight independently significant predictors from diagnostic, clinical, psychosocial and treatment history domains: Substance use disorder diagnosis; medication side effects; moderate to severe psychotic symptoms; personality disorder diagnosis; economic problems; prior hospitalisation; current Global Assessment of Functioning scale score and duration of treatment from current psychiatrist (Compton, 2007). The summarised results of Compton's (2007) review are featured in Table 1 (below).

Table 1: Risk factors for non-adherence to antipsychotic medications and follow-up appointments amongst people with schizophrenia (from Compton, 2007).

NOTE:

This figure/table/image has been removed to comply with copyright regulations. It is included in the print copy of the thesis held by the University of Adelaide Library. 
There appear to be several factors that are consistently proposed to influence adherence in the literature, which are, thus, consistently tested in studies. Although these factors are often labelled differently or grouped under different broad categories between studies, they are nonetheless referring to the same or similar phenomena. The factors that were consistently associated with adherence and/ or assessed for their association with adherence are frequently classified as consumer factors, illness factors, medication factors, service factors and social factors. Consumer factors typically refer to demographic factors which are consistently tested despite limited support for their association with adherence (i.e., Fenton, Blyler \& Dubin, 1997; Lacro et al., 2002). Other commonly raised consumer factors include consumer attitudes towards medication, previous substance abuse or dependence (although this is sometimes considered a social factor) and forgetfulness (although this is sometimes considered an illness factor related to the cognitive impairments associated with schizophrenia). Regarding illness factors, insight has consistently been found to be one of the strongest predictors of adherence. Other illness factors frequently assessed and sometimes associated with adherence include the severity of psychopathology generally, in addition to the severity of specific symptoms including paranoia, grandiosity and hostility. Medication factors included the consumer's response to medication in addition to its tolerability and the presence of side effects and adverse reactions to medication. The dosage, route, regimen and administration of medication, in addition to the type of medication (typical or 
atypical) are often assessed for associations with adherence. Service factors, including the therapeutic alliance between the prescriber and the consumer, have commonly been assessed. Social factors such as family support and stigma are also often assessed in relation to adherence.

\subsubsection{Qualitative research related to adherence}

Whilst the value of quantitative research in relation to medication adherence amongst people with schizophrenia cannot be disputed, it has typically involved measuring the influence of pre-determined sets of factors. Thus, quantitative research has not shed any light on how various factors influence adherence and how factors may interact.

As Rogers et al. (1998) point out, despite the importance attributed to antipsychotic medication adherence amongst people with schizophrenia, little attention has been paid to the consumer perspective. This gap may reflect a perception that people with schizophrenia are irrational and, therefore, incapable of offering a valid viewpoint, despite research which has demonstrated that people with schizophrenia are able to make accurate assessments about the effects of their medication (e.g., Day et al., 1995). Like the research presented in this thesis, the following studies (which represent the extant literature in the area) applied consumer-focussed approaches to understand medication adherence amongst people with schizophrenia. Qualitative data derived from the personal accounts of people diagnosed with schizophrenia were analysed and were sometimes triangulated with the views of relative caregivers and clinicians. Notably, some repetition of factors explored in some of the quantitative research that was discussed is apparent. In some instances, interview plans directly explored pre-established factors. 
Nonetheless, by providing scope for consumers to discuss factors, the following research facilitates a deeper understanding of adherence.

Rogers et al. (1998) analysed data from semi-structured, in-depth interviews that explored reasons for taking antipsychotic medications and the ways in which they self-regulated their medication. The sample consisted of 34 people who had been diagnosed with schizophrenia or schizo affective disorder and prescribed a regimen of long-term antipsychotic medication (oral and depot) from the United Kingdom. Interviews focused specifically on conceptions of the cause and nature of schizophrenia, experience and knowledge about medications, the nature of medication practices used on a day-to-day basis, management strategies, the involvement of others in managing medication and attitudes towards professionals and although not articulated, the analysis appeared in line with a thematic analysis approach. For most participants, adherence was associated with recognition of the benefits of medication (such as symptom control and relapse and hospitalisation prevention) and/or the personal costs associated with nonadherence. The main utility of medication was seen as its ability to act directly on symptoms by stopping them or reducing them, rendering them more manageable. Participants identified one of the costs associated with taking antipsychotic medication as the experience of side effects, which at times, equalised or outweighed the positive gains associated with taking medication. There was evidence that participants had acquired knowledge regarding the levels of medication they needed to control their illness and, beyond those levels, had gained some freedom to adjust the medication to manage side effects and other problems involved. That is, whilst two thirds of the participants reported to take their medication as prescribed, some selfmedicated by increasing or decreasing the dose or taking medication at a 
different time of the day. In addition to several adaptive strategies to assist illness management, self-medication with non-prescription substances was also a reported method of coping with positive symptoms and the side effects of antipsychotic medications. Spirituality and resources which promoted autonomy, such as money, friends and independent living, were cited as ameliorating factors. Medication taking was frequently perceived to be in the control of others and influenced by a range of social sanctions. Significant others were reported to prompt or remind participants to take their medications, which was typically not described as impinging on their autonomy. However, many participants attributed adherence to awareness of health professionals' abilities to implement legal and social consequences for non-adherence, which can include the loss of liberty for consumers (i.e., being compulsorily detained in hospital) (Rogers et al., 1998).

Carrick, Mitchell, Powell, \& Lloyd (2004) conducted an interview study that investigated individuals' experiences of the side effects of antipsychotic medication. The sample comprised of 25 outpatients from Exeter, South West England, and including a focus group who were recruited through day centres. Interview data were analysed based on a grounded theory approach. The analysis reported that consumers did not perceive side effects and symptoms as separate issues. Rather, their positive or negative evaluations of medication related to the total impact of medication treatment on their functioning, feeling and appearance to the outside world (Carrick et al., 2004). Rogers et al. (1998) similarly reported that the ways in which physical and psychological side effects acted to inhibit everyday social interaction and opportunities were particularly important for a number of participants' adherence. 
Sharif, Ogubanjo and Malete (2003) conducted a qualitative study aimed at enhancing understanding of reasons for treatment non-adherence amongst consumers with mental illnesses, including schizophrenia, from a rural South African province. Six non-adherent consumers were identified from clinical records and interviewed. A family member of the consumer was also interviewed. Interviews of consumers and their family members were paired, then interviews were analysed for codes and integrated into a single model following a grounded theory approach. Multiple causal factors were identified to be associated with consumers' non-adherence. Side effects were the most common reason for non-adherence, mentioned by almost all of the participants and confirmed by their caregivers. In some cases, participants indicated that they preferred the experience of illness symptoms to side effects. It was additionally found that none of the participants had been informed about side effects, possible remedies and coping mechanisms prior to commencing pharmacotherapy. Some participants stated that they discontinued medication as it was ineffective in treating symptoms. One consumer also expressed a fear of receiving depot medication, which he felt made him ill, thus, accounting for non-adherence. A lack of family support was also identified by three of the participants as an influence on adherence. Furthermore, one caregiver reported that they had attempted to coerce a consumer into taking their medication, which lead to resistance and nonadherence (Sharif et al., 2003).

Lack of continuity of care was constructed as a deterrent to treatment for some consumers, who elaborated that they were required to tell their story repeatedly to new staff. Additionally, half of the participants reported a lack of ongoing assessment, such that once their symptoms were controlled, they were not reassessed according to changing contexts or on the basis of the 
changing magnitude of their illnesses. In most cases, participants and their families expressed frustration in relation to their non-involvement in management plans and the lack of information given to them in relation to the nature of illness and the pattern of therapy. Autonomy and ego, in particular, the desire for independence and self-control, exerted a pivotal role in most of the participants' accounts of non-adherent behaviour. This was particularly relevant to cases when participants were not involved in their illness management and the treatment approach was confrontational and coercive, which participants reportedly responded to with rebellion and resistance to attempts to control their lives through medication. Gains related to the sick role were also attributed to non-adherence. Specifically, two consumers and their family members reported that they became nonadherent in order to be eligible for disability pensions (which had cancelled when their illness symptoms were controlled as a result of adherence). Adverse social stigma was also associated with non-adherence to treatment amongst some participants, who reported feeling embarrassed by their "mentally ill" identities and, thus, failed to attend appointments. Different belief systems were also proposed to influence adherence, such as a belief that witchcraft or stress was the underlying cause of the illness (Sharif et al., 2003).

Despite the interesting findings of this study (Sharif et al., 2003), there are concerns regarding the interpretation of interview data. In particular, the article did not identify who conducted interviews but it was reported that they were translated from participants' first language, Tswana, into English once completed. Furthermore, the study's sample size of six was extremely small, even for a qualitative study. Whilst a purposive sampling approach was selected to ensure variation in medication regimen, gender and marital 
status, the selection of only non-adherent consumers and the small sample size hinder the generalisability of study findings.

Carder, Vuckovic and Green (2003) conducted qualitative interviews with 83 adults with chronic illnesses, including schizophrenia and schizo affective disorder, which investigated consumers' perceptions of their need to take medication during the course of their illnesses. Unfortunately, it was not noted how many of the participants had schizophrenia or schizo affective disorder. Semi-structured interviews were transcribed and analysed following a grounded theory approach. Participants in the study described a range of responses to their medications. For some, medications successfully managed their symptoms whilst others reported ongoing efforts to find the right medication, or combination of medications, to manage symptoms and minimise side effects. Most described changes over time, with periods of stability marred by either medication resistance or side effects that required a change in dose or type. The results of the study indicated that participants negotiated their need for medication internally (including struggles over self identity) and externally (through negotiations with health care providers). Interview data indicated that medication taking may prompt consumers to re-negotiate their self-identities as formerly well persons (Carder et al., 2003).

Consumers may, thus, question their illness diagnosis. When symptoms are under control, they may question whether they are cured, in remission, or if the medication is treating symptoms. Some participants resisted taking medication because it conflicted with their identities as a healthy person or someone who normally did not take medication. Some participants stated that they reduced their intake of medication to curtail side effects or discover the dosage that best met their personal threshold for 
symptom management. Regarding external negotiations, participants described both battling and working with their physicians over medications, including decisions regarding whether to take medication, type of medication, how much and by what route. Many of the participants had taken medication for years and, thus, knew what worked and did not work for them. One source of resistance derived from participants' dissatisfaction with physicians who simply prescribe medications whenever the individual has new or additional symptoms, leading to complex medication regimens. In addition to the physical effects of taking medication for an extended period of time, some participants reported an emotional toll associated with the trial and error involved in finding the right medication regimen (Carder et al., 2003). Indeed, two participants with schizophrenia reported feeling like a "human experiment" as a result of the long process of finding the right medication or combination of medications (Carder et al., 2003).

More recently, Shoemaker and Ramahlo de Oliveira (2008) conducted a study focussing on the meaning of medication for 41 consumers, which included participants with diagnoses of schizophrenia (as with the previous study, the number of participants with schizophrenia was, unfortunately, not reported). A meta-synthesis of three different but complementary qualitative studies was conducted by researchers, which included unstructured and indepth interviews as parts of phenomenological and ethnographic studies. The authors defined the medication experience as an individual's subjective experience of taking medication in their daily life. The meaning of medication was captured by four codes of the medication experience: a meaningful encounter; bodily effects; unremitting nature; and exerting control, which the authors considered reflected stages of the medication experience. 
According to the authors, the experience begins when a consumer first encounters medication, which is typically embedded with meaning before it occurs. The meaningful encounter can be revealed as a sense of losing control, a sign of ageing or a signifier of illness, and often causes questioning and a meeting with stigma. Whilst questioning the need for medication upon diagnosis is typically interpreted as resistance by health care professionals, the authors propose that for participants, it can represent a means of regaining a degree of control. Participants sensed that their individual autonomy was undermined when taking chronic medications until the point that they questioned the taken-for-granted notion that medications are the right option. The first reactions to initiating a medication regime can also be shaped by the social views of the medical condition, including stigma. Bodily experiences refer to the effectiveness of medication and side effects. The bodily effects of medications code was revealed as the experience of a "magic elixir" or trade-offs. Some participants indicated that medication could "normalise" them, whereas others indicated that medication alleviated them from incapacitation and, thus, enabled them to function. Participants who experienced side effects were willing to accept them as a trade-off if the benefit experienced by medication was sufficiently good. The unremitting nature of a chronic medication is considered a burden and participants often recalled that they responded angrily to this realisation. The expectation of taking medication regularly positions the patient as a passive agent and the medication as a symbol of dependence. The last code of the medication experience was revealed as consumers exerting control over their medication. After encountering the meaning of a medication, questioning it, realising the bodily effects and the continuous nature of medications, participants experimented with becoming the managers of their treatment regimens. 
After taking medications for a period of time, participants became familiarised with the effects of medication on their bodies. They discovered creative ways to manage their medications and exert control over them; in part because they were now knowledgeable (Shoemaker \& Ramahlo de Oliveira, 2008, 2008).

The views of consumers, carers and professionals from four different countries (England, Germany, Italy and the Netherlands) were combined in a study by Kikkert et al. (2006) which explored adherence amongst people with schizophrenia who had been prescribed antipsychotic medication for at least a year, using the qualitative method of concept mapping. The sample comprised of 27 people with schizophrenia, 29 carers and 28 professionals involved in the treatment of consumers. Participants were allocated selectively to groups which were comprised of members of each stakeholder group. The concept mapping procedure involved group discussions about factors that influence medication adherence. Brainstorming of factors related to adherence was conducted amongst groups and participants were asked to generate statements about influences on adherence. These statements were then reviewed by researchers and reduced to a manageable number via the combination of similar statements and deletion of statements regarded as least relevant. Individual group members then conducted clustering; the process of determining which factors emerged from the data and to what extent factors related to each other. This was followed by prioritising, or ordering of the data in terms of importance in relation to medication adherence. Ultimately, five clinically relevant codes were identified as affecting adherence: medication efficacy, external factors (such as patient support and therapeutic alliance), insight, side effects and attitudes towards medication. Interestingly, the importance of each of these factors differed 
significantly between consumers, carers and professionals, indicating that these stakeholder groups do not have a shared understanding of what influences medication adherence behaviour. The greatest disparity in prioritising was observed between consumers and professionals (Kikkert et al., 2006).

Kikkert et al.'s (2006) finding in relation to disparities between consumers and professionals regarding the degree of influence of factors on adherence has also been supported by Rogers et al. (1998) who pointed out that in previous studies, psychiatrists have over-estimated the proportion of non-adherence explained by illness factors, such as insight, particularly when compared to consumer perspectives. According to Rogers et al. (1998), this reflects the difference between the way that non-adherence is perceived amongst people with physical conditions compared to those with schizophrenia. In the latter case, non-adherence is viewed as an illness symptom and, thus, negative attitudes to medication are seen as irrational and a direct consequence of the underlying psychoses.

Despite the different study populations and research designs, some consistencies emerged across qualitative research related to adherence amongst people with schizophrenia. For example, all of the studies used qualitative interviews as the means of data collection. Adherence was frequently associated with the benefits of medication in terms of symptom control and the costs associated with non-adherence (such as relapse and rehospitalisation) (Rogers et al., 1998). Consumers generally talked about the effectiveness of medication in treating symptoms and the side effects associated with medication collectively and weighed the two against each other to determine their attitudes towards medication (Shoemaker \& Ramahlo de Oliveira, 2008, 2008). Functioning and appearance to the 
outside world were important considerations for consumers when discussing the benefits and costs of medication (Carrick et al., 2004). Once consumers acquired knowledge about their illnesses and medication, which typically occurred with time and experience, they reported to engage in more practices akin to self-medication, such as reducing their dosage of their own accord (Shoemaker \& Ramahlo de Oliveira, 2008, 2008). Thus, in some instances, non-adherence can represent a means of expressing control over their treatment. Healthcare providers were generally viewed as exerting a significant amount of control over consumers' treatment and a lack of involvement of consumers and family members in managing treatment was reported. Additionally, there were significant disparities between the views of consumers and healthcare providers regarding the relative impact of influences on adherence (Kikkert et al., 2006), highlighting the importance of paying attention to the consumer perspective so that interventions can target appropriate risk factors.

A limitation of two of the studies discussed was that they synthesised the findings from studies that explored the medication experience across multiple chronic conditions including schizophrenia, rendering it difficult to discern the aspects of participants' experiences related to the unique illness experience of schizophrenia. Additionally, interview plans were occasionally based on factors previously established in the literature to influence adherence, thus, potentially accounting for the emergence of some codes. Furthermore, none of the studies identified occurred within an Australian setting and thus, may be limited in their applicability to Australian populations, whereby the health care system and medications that are available may differ. The research presented in this thesis aims to address these limitations. Interviews only involved people who met diagnostic 
criteria for schizophrenia, were semi-structured and contained general questions.

\subsection{Chapter summary}

The term 'adherence' refers to the extent to which consumers' behaviour matches agreed recommendations with the prescriber. It has been used in the present study because it implies greater agency for consumers in relation to their illness management than previously used terminology, such as 'compliance'. Medication adherence amongst people with schizophrenia is consistently shown to be associated with positive outcomes, including symptom reduction, reduced relapse rates and improved general functioning. By contrast, non-adherence (including partial non-adherence) is frequently associated with symptom fluctuations and higher risks of relapse and hospitalisation. Despite the established knowledge that poorer outcomes are associated with medication non-adherence, rates of non-adherence are amongst people with schizophrenia remain high. Although true rates of adherence are difficult to quantify and there have been inconsistent findings, non-adherence has been estimated to be between 30 and $75 \%$ for oral medications. Lower rates of non-adherence amongst consumers prescribed depot medications could be explained by a greater number of consumers who take depot medication being on community treatment orders.

Factors consistently proposed to influence adherence in quantitative studies include insight, substance use, consumer attitudes towards medication, the relationship between the prescriber and consumer, efficacy of medication and side effects. Although considerably less qualitative research has been conducted specifically focusing on adherence amongst people with 
schizophrenia, those studies that have been conducted have produced enlightening results and revealed significant disparities between consumer and healthcare provider perspectives regarding the relative importance of various factors on adherence.

Given that the present study aims to assess influences on medication adherence, some overlap with previous findings occurred. Reviews converge in concluding that non-adherence is better documented than understood and that a focus on individual consumers' decision-making process is often an important, missing element in quantitative research (Fenton et al., 1997). In addition to offering scope for the elucidation of factors not previously researched in the literature, the present study, through its qualitative, interview design, aims to build on our understanding of medication adherence. Justification for, and elaboration of, the methodology of the research presented will be provided in the following chapter. 


\section{Chapter 4: Methodology}

The purpose of the qualitative research presented in this thesis was to explore the experiences with antipsychotic medication of outpatients with schizophrenia. A secondary purpose of the study was to illuminate consumers' attributions to their subjective adherence or non-adherence, past or current, in order to gain an insight into the types of factors that influence adherence amongst this population. This chapter outlines the methodological procedure undertaken for the research presented in this thesis. Qualitative in approach, the research involved one to one, semistructured interviews with consumers that focused on their experiences of taking antipsychotic medication. Interviews were then transcribed and analysed according to the principles of grounded theory. Whilst the analysis was informed by a grounded theory approach, a full grounded theory analysis, involving the generation of a theory, was outside the scope of the thesis. This chapter begins by providing a justification for the research followed by a discussion of grounded theory. Subsequently, relevant ethical considerations are addressed and the sample, data collection and data analysis are described.

\subsection{Why use a qualitative research approach?}

As emphasised in prior chapters, a review of the literature on medication adherence amongst people with schizophrenia yielded relatively little research from the consumer perspective. Some valuable qualitative research has been conducted (as outlined in Chapter 3) however, few studies 
were found that focused specifically on adherence amongst people with schizophrenia. Furthermore, interview plans used in the qualitative studies described typically focused on factors established in the literature to influence adherence. Additionally, none of the studies reviewed were conducted in Australia. Thus, the research presented in this thesis will contribute to strengthening existent qualitative evidence.

As previously mentioned, whilst medication adherence amongst people with schizophrenia represents a topic of extensive research, the majority of published studies in the field have incorporated quantitative designs. Whilst such quantitative studies require the participation of consumers, they preclude the identification of consumers' own ways of making sense of medication adherence by not providing consumers with opportunities to express their subjective experiences of medication adherence. Whilst the utility of the results of quantitative studies in contributing to our knowledge of medication adherence is not disputed here, such designs afford limited (if any) scope for information beyond that which is being directly measured to be communicated. Through its reliance on hypotheses generated by existing theories, quantitative adherence research has, thus, foreclosed the possibility of generating completely new theories on adherence; in particular, theories from the perspectives of consumers (Willig, 2001). It is highly likely that previous quantitative research has failed to capture the complexity of medication adherence amongst people with schizophrenia, potentially overlooking important influences on adherence which could then be addressed through intervention.

One rationale for qualitative research is to expand our theoretical understanding of phenomena, which depend on the context-specific collection and interpretation of richer and deeper forms of data (Rogers et al., 
2003). According to Hesse-Biber and Leavy (2004) and Ezzy (2002), qualitative researchers are motivated to understand the world of the research participants through the eyes of participants, as human behaviour, unlike that of physical objects, cannot be understood without reference to the meanings and purposes attached by humans to their activities. Whilst it is acknowledged that there are multiple forms of qualitative research, Jones, Torres and Arminio (2006) summarise the intent of qualitative research as to illuminate and attain a deeper, improved understanding of the rich lives of humans and the world. It is acknowledged that there are a multiplicity of forms of qualitative research, which do not all have the same rationales, however. By utilising a form of qualitative analysis here, this research addresses the limited voices of consumers in adherence research in the literature.

Qualitative data are non-numerical and are usually obtained from unstructured or semi-structured research methods (Hesse-Biber \& Leavy, 2004). Consistently, the current research involved conducting in-depth, semi-structured interviews with outpatients with schizophrenia. Interviews explored consumers' understandings of the meanings and uses of medication, potential obstacles and incentives to adherence, useful strategies to facilitate adherence as well as other topics spontaneously raised in relation to the topic, with the ultimate aim of gaining an insight into interviewees' lives. Interviews were audio-recorded, then transcribed and analysed. Analysis remained close to the data, in order to provide a rich, descriptive account of experiences. A more detailed account of the analytic procedure will be provided later in this chapter. Although some overlap with previous findings occurred, research findings added to the qualitative research in the area and helped to contextualize the ample statistical data available on adherence. 
Furthermore, the present research elucidated influences on adherence not yet established in the literature and, thus, represents a valuable contribution to knowledge about medication adherence amongst people with schizophrenia.

\subsection{Study design}

This research draws on the principles of grounded theory, however, the analysis more closely resembles a thematic analysis as a process model or theory of medication adherence was not developed. The method of data collection was face-to-face, semi-structured interviews with outpatients with schizophrenia who had experiences with taking antipsychotic medications. Interviews were conducted at day centres and medication clinics in the Northern and Eastern metropolitan areas of Adelaide and at a public hospital in Northern metropolitan Adelaide. Interviews were conducted between $31 / 07 / 2008$ and $25 / 2 / 2009$, and varied in length from 30 minutes to approximately two hours. Further details of the study procedure will be provided below.

Qualitative interviews were initially conducted with a view to designing an intervention to assist with medication adherence, based on interviewees' responses. Upon analysis, however, it became clear that the data did not lend themselves to a clear-cut intervention. This was partly because the meaning of adherence was challenged. Furthermore, data highlighted the inter-subjectivity of medication experiences amongst consumers and interviewees frequently directly opposed one generalised intervention to address adherence on these grounds. 


\subsubsection{Grounded theory methodology}

Grounded theory emerged in the 1960s against a climate of longstanding debates in the human sciences about the relative merits of qualitative and quantitative inquiry (Pidgeon, 1996). Indeed, the developers of grounded theory, Barney Glaser and Anselm Strauss, were schooled in opposing research traditions. Glaser studied in the department of sociology at Columbia University which was renowned for a reliance on quantitative methodology whereas Strauss was schooled in the Chicago tradition, which was associated with down-to-earth, qualitative research (Glaser \& Strauss, 1967). In 1967, a critical point in social science history, Glaser and Strauss co-authored the book, "The Discovery of Grounded Theory: Strategies for Qualitative Research". At the time, there was an overwhelming preoccupation with the quantitative testing of propositions derived from a few, highly abstract "grand" theories. This lead to impoverished theory, with limited relevance to any substantive content domain and compromised the scope for the emergence of new theories (Pidgeon, 1996). Glaser and Strauss' grounded theory, thus, served at the front of the qualitative revolution (Charmaz, 2003). In their book, Glaser and Strauss challenged the dominant view that quantitative research represents the only form of systematic social scientific inquiry. More specifically, they attempted to bridge the gap between theory and empirical research; to generate theory that is relevant to research (Glaser \& Strauss, 1967). In summary, through grounded theory, Glaser and Strauss proposed a radical philosophical shift, aimed at generating more local, contextual theory that would, as a consequence, be of relevance to those studied (Pidgeon, 1996).

A grounded theory approach to inquiry involves intimate engagement with the data which typically results in the generation of a theory that is 
grounded in the data. The resultant theory is inductively developed during a study and in constant interaction with the data from that study (Ezzy, 2002; Maxwell, 2005). This theory is grounded in the actual data collected, in contrast to theory that is developed conceptually and then simply tested against empirical data. It does not rely exclusively on established views or the researcher's own perspective, as is typical of quantitative research (Maxwell, 2005). Nonetheless, it is acknowledged that the researcher enters into the process with their own, personal preconceptions and inevitably influences the data obtained through the data gathering process (i.e., interview questions). Grounded theory draws attention to the perspectives of research participants, including their subjective accounts of social and psychological events and of associated phenomenal and social worlds (Maxwell, 2005; Pidgeon, 1996; Rubin \& Rubin, 1995). The ultimate purpose of grounded theory is to develop a theory that remains close to, and illuminates, the phenomenon under investigation by studying the experience from the standpoint of those who live it (Jones, Torres \& Arminio, 2006).

Grounded theory was deemed a suitable approach for the current study due to its commitment to communicating the participant perspective. I believe that the consumer perspective on medication adherence provides a valuable contribution to knowledge in the area, particularly because of the complexity of medication adherence and the failure of health services to address medication adherence effectively amongst people with schizophrenia on a large scale despite the extensive research in the area. With its openness to generating theory which has not necessarily been pre-established in research, I perceived a grounded theory approach to the topic of medication adherence as potentially groundbreaking as well as valuable both in academic and practical terms, with potential clinical implications (Rubin \& Rubin, 
1995). Although the research presented was influenced by a grounded theory approach, however, the analysis did not ultimately involve theory generation as this was beyond the scope of the thesis.

Grounded theory methods have become a topic of debate from both proponents and opponents of the approach. Post-modernists and poststructuralists dispute the positivistic premises assumed by grounded theory's major supporters and within the logic of the method itself (Charmaz, 2003). The positivistic assumptions of grounded theory stem from the reliance on a realist ontology, which posits that there is a "real", objective reality that researchers are able to directly and, therefore, objectively and accurately capture and represent (Willig, 2001). There has also been divergence in the grounded theory methodology between Glaser and Strauss (in collaboration with his more recent co-author, Juliet Corbin), who have developed the grounded theory method into conflicting directions, leading to a split between Glaserian and Straussian grounded theory. Glaser's position is close to traditional positivism, as it assumes an objective, external reality. Furthermore, the researcher is positioned as a neutral observer who discovers data, reduces inquiry to a set of manageable research questions and objectively renders data (Charmaz, 2003). Strauss and Corbin's position assumes an objective external reality, aims toward unbiased data collection, proposes a set of technical procedures and supports verification (Charmaz, 2003). Strauss and Corbin's stance is aligned more with post-positivism, however, as it additionally advocates giving voice to participants, representing them as accurately as possible, discovering and acknowledging how participants' views of reality may conflict with researchers' and recognizing creativity as well as science in the analytic product and process (Charmaz, 2003). 
In the research presented, participants' accounts are treated as real, however, the impossibility of being able to represent the consumer perspective exactly as it occurs in reality is acknowledged. As the primary researcher, I aimed to be reflexive throughout the conduction of the research presented. Later in this chapter, I disclose details that relate to my background (see 4.3.2 Ethical considerations), in addition to the various ways in which I may have influenced the results of this study. As acknowledged earlier, whilst the research presented was influenced by a grounded theory approach, a process model of medication adherence as part of the analysis was not produced as this was beyond the scope of the thesis.

\subsubsection{Ethical considerations}

Ethics approval was attained to undertake the study from the Central Northern Adelaide Health Service Ethics of Human Research Committee (covering the Queen Elizabeth Hospital and Lyell McEwin Health Service). The research procedure of one-to-one interviewing was unobtrusive. Participation was completely voluntary and participants were free to withdraw from the study at any time prior to the completion of interviews. As the primary investigator, I distributed information sheets to potential participants for their perusal. Potential participants were encouraged to discuss the study and share all documents with other members of the public, such as family members, peers, case managers or health workers prior to deciding whether to participate or not. Upon agreeing to participate, prospective interviewees were given a consent form to sign and were then screened to ensure they met the requirements for the study. The audio files were recorded during interviews and then I transcribed them. Transcriptions were transferred into a study database to allow the results of this study to be 
analysed and reported. Data were stored on a personal computer and a USB drive. I was the only person with access to the audio recordings of transcripts. Respondents were assured that their identities (and the identities of other people discussed in interviews) would remain confidential as no identifying information would be included in the write-up.

Pseudonyms were created for participants (and other people discussed, such as prescribers) to help to preserve their anonymity and other identifying information was changed or excluded from transcriptions. Attempts were made to match pseudonyms to participants' genders and ages. Information provided by participants in interviews was only used for the purpose of the study.

\subsection{The sample}

\subsubsection{Recruitment strategy and sampling frame}

Outpatients were recruited from mental health outpatient and rehabilitation services, consumer organizations and a medication clinic. The initial recruitment strategy involved distributing flyers to various outpatient services, which was ineffective in attracting participants (see Appendix A for

example flyer). Approaching potential participants was much more effective in the early stages of recruitment, with the assistance of a research nurse. Presenting my research to outpatient groups and asking for expressions of interest in participating also proved an effective means of recruitment. The research nurse was of great assistance as she had contact details of several consumers who were willing to participate in research as they had done so in the past. 
The sampling occurred in two phases, with the first being a purposive approach, whereby a particular sub-set of people were required and selected deliberately for interviews in order to provide the information needed to address the research question (Maxwell, 2005). Those who did not match the criteria were excluded from the study. The research nurse facilitated this process significantly, through identifying relevant contacts or by recognizing potential candidates in settings (such as the medication clinic) where I was unable to. Snowball sampling then occurred naturally as many interviewees stated that they enjoyed interviews and, thus, agreed to distribute information sheets to peers who met the study requirements. As my details were listed on the information sheet (see Appendix C), I was then contacted by consumers and interviews were arranged. Recruitment ceased following theoretical saturation, when I noticed consistent repetition of codes and no new conceptual insights were generated (Bloor \& Wood, 2006). I decided that I had reached theoretical saturation in consultation with my supervisors. This was accomplished after 22 interviews. Two more interviews were conducted after this to ensure that saturation had been achieved.

Of note, the grounded theory principle of theoretical sampling was not adhered to. Theoretical sampling refers to the purposive selection of research participants to compare with prior cases in order to gain a deeper understanding of analysed cases (Glaser \& Strauss, 1967). Sampling is, thus, based on emerging codes and categories until a full and varied category is developed and tested against incoming cases. All participants in the research presented were outpatients with schizophrenia and exclusion criteria were minimal. As interviewees' experiences were so varied and they were asked to reflect on their experiences at different stages of their illnesses, theoretical sampling was deemed unnecessary. Although it could be argued that 
inclusion of service providers views, for example, may have broadened the theory, this would have been inconsistent with the focus of this research; the consumer perspective.

\subsubsection{Inclusion and exclusion criteria}

For inclusion in the study, participants had to: meet DSM-IV-TR criteria for a diagnosis of schizophrenia; be aged between 18 and 65 years; be able to communicate effectively in the English language; be an outpatient and have been prescribed antipsychotic medication (typical or atypical medications including clozapine, oral or intravenous administrations, varied dosages) by a health professional to treat their schizophrenia. Of note, it was found during screening for entry into the study, that some people who had been given formal diagnoses of schizoaffective disorder also matched the criteria for schizophrenia and were, therefore, included in the study. Participants were also required to sign a consent form prior to taking part (see Appendix D).

The exclusion criteria for this study were intellectual disability and severe co-morbid conditions (such as drug dependence which could hinder capacity to interview). Although more exclusion criteria were originally proposed (e.g., people on treatment orders or taking depot antipsychotic medication), these were later removed as it was decided that variations in interviewee responses due to such criteria could provide interesting insights into adherence. Furthermore, the original exclusion criterion of people being prescribed typical antipsychotic medication was also removed as it was decided that this could potentially render irrelevant interesting discussions about past experiences with medications amongst interviewees who were previously prescribed typical antipsychotic medications. Furthermore, the 
views of consumers who continue to be prescribed typical medications are considered just as important as those who are prescribed atypical medications, particularly considering that there are adherence difficulties associated with both types of medication.

\subsubsection{Selection of participants}

As previously mentioned, participants were screened to ensure they matched the criteria for schizophrenia using the DSM-IV-TR criteria. As primary researcher, I administered the screen prior to interviews. The screening process was tested on two pilot interviewees and on some peers who did not have a previous diagnosis of schizophrenia and it proved effective. The same approach had previously also been used effectively by a fellow student examining cognition amongst people with schizophrenia.

\subsubsection{Demographic information}

Demographic information was collected from participants prior to interviews. This helped to establish rapport and to ease interviewees into the interview process. The demographic characteristics of the sample are presented in Appendix E. A sampling frame was not devised prior to interviewing, however when it became noticeable that certain groups (i.e., gender groups) were being represented disproportionately to others, sampling became more purposive to address this. That is, more females were approached to participate.

In the end, males were represented more than females in the sample ( $M=15, F=10)$ however this could also be a reflection of higher incidence of schizophrenia amongst males (i.e., McGrath et al., 2004). Ideally more young participants would have been involved (the youngest participant 
included was 19 and the second youngest was 25). A broad age range of participants was interviewed however (19-65 years). The mean age of participants was approximately 40.8 years and the median age was 38 years.

Additional demographic information was obtained, including participants' age of illness onset, which ranged from 16-57 years of age. The average age of illness onset according to participants was approximately 27.5 years. A few participants had relatively late illness onsets (45, 47 and 57 years), however, which may have skewed the average calculation. The median age of onset, estimated as 22.5 years, may more accurately reflect the sample and is consistent with literature which indicates that schizophrenia symptoms typically emerge in adolescence and early adulthood (Smith et al., 2009; Van Os \& Kapur, 2009).

The number of hospitalisations experienced by participants ranged from 1 to 30. Notably, four participants indicated that they had been hospitalised too many times to recall the exact number. Ten participants resided alone whereas the remaining 15 lived either with family, a partner or in a share-house situation. The majority of participants were prescribed medication by their psychiatrist (21) and the remainder were prescribed medication by General Practitioners.

The majority of the participants reported that they were prescribed atypical medications at the time of interview, however, two participants were prescribed typical antipsychotic medications and one participant was prescribed two typical medications and one atypical medication. Indeed, seven participants were prescribed more than one antipsychotic medication. risperidone was the most commonly prescribed medication amongst the 
sample. Four participants were prescribed clozapine. Although the majority of participants took their medication orally, four received depot injections (which could explain the high number of participants who were prescribed risperidone considering it is available in oral and depot form).

\subsection{Data collection}

\subsubsection{Semi-structured interviews}

Face-to-face, semi-structured, in-depth interviews were used to collect data. The deployment of in-depth interviews to gather data assumes that individuals have a unique and important knowledge about the social world that can be ascertained through verbal communication (Hesse-Biber \& Leavy, 2006). According to Fylan (2005), interviews are one of the most popular means of data collection in qualitative research as they enable researchers to talk to people and find out about what they have experienced and what they think and feel about a topic of interest. Qualitative interviewing is characterized by open questions that elicit stories and case-oriented narratives and should strive to preserve the multi-vocality and complexity of the lived experience (Hesse-Biber \& Leavy, 2004).

A semi-structured approach, as opposed to a structured approach to interviews was selected as structured interviews run the risk of phrasing the researcher's own concerns into the mouths of respondents, thereby limiting the voice, perceptions and meaning-making around the research topic of the interviewee (Hesse-Biber \& Leavy, 2004). Semi-structured interviews rely on a set of questions that loosely guide the conversation but also allow interviewees the latitude and freedom to talk about what is of interest or 
importance to them. The conversation is thus free to flow naturally, making room for it to travel in new and unexpected directions (Hesse-Biber \& Leavy, 2006). Due to their flexibility, semi-structured interviews have been proposed as an effective means of elaborating "why" questions, rather than how much or how many, rendering them particularly malleable to the present study which focuses on why people with schizophrenia take or do not take their medication (Fylan, 2005).

Hesse-Biber and Leavy (2006) recommend semi-structured interviews as tools for accessing subjugated voices and, thus, gaining access to subjugated knowledge while simultaneously challenging dominant powerknowledge relations. Those marginalized in society, such as mentally ill consumers in the present study, are likely to have hidden experiences and knowledge that has been excluded from the general understanding of social reality. This holds true in particular for people with schizophrenia, whose voices have largely been neglected in literature about medication adherence. Given that the aim of this research is to develop a theory on adherence from the consumer perspective, semi-structured interviews were deemed the ideal means of data collection.

All interviews were conducted one-to-one, with the exception of the first interview which involved two consumers who were in a relationship. During this (pilot) interview, I found it difficult to focus on interesting information raised by individual interviewees and also noticed that interviewees would occasionally speak for each other, cut each other off and influence each other's responses, hindering the depth of information attained. Reflecting on this interview, following listening to and transcribing it, I decided to only conduct one-to-one interviews thereon. 


\subsubsection{Interview schedule development}

An interview schedule was developed based on a set of topical areas and questions and represented the framework for the interview (Hesse-Biber \& Leavy, 2006). According to Maxwell (2005), qualitative researchers undertaking semi-structured interviews should pilot test their interview schedules with people as close to their planned interviewees as possible, to determine whether the questions work as intended and what revisions may need to be made. Consistently, potential interview questions were tested on inpatients and outpatients during hospital visits and day centre visits respectively, in order to familiarize myself with the population I would be interviewing, the service and interview settings, as well as the language used by consumers. My experiences during these visits were documented in field notes and were crucial in developing the interview schedule. Questions were framed in terms easily understandable by interviewees and were re-worded according to contextual factors. I was mindful of the value of asking interviewees 'real' questions, that I was genuinely interested in the responses to, rather than contrived questions designed to elicit particular kinds of data whilst developing my interview schedule. This reflected an attempt to create a more equal and collaborative dynamic, in which interviewees were able to bring their own knowledge to bear on the questions in ways that I had not necessarily anticipated (Maxwell, 2005). Some examples of questions that were included in the schedule are: What influences you to take/not take your antipsychotic medication? What are some of the benefits associated with antipsychotic medication? What are some of the negative aspects of antipsychotic medication? (see Appendix B for interview schedule). 


\subsubsection{The interview process}

Interviews took place in interview rooms at the Lyell McEwin hospital, a medication clinic and day centres. Interviews ranged in length from approximately half an hour to two hours. Following the collection of demographic information, an opening question that overviewed the topic and set the stage for the interview, was typically posed (although worded differently each time, the question was usually something along the lines of: Can you please tell me about your experiences with taking antipsychotic medication?). Sometimes referred to as the "grand tour" question, this question was designed to elicit narratives detailing interviewees' conceptions of the identified domain (Hesse-Biber \& Leavy, 2004). There was no fixed ordering or wording of questions (except in the demographic information section). Questions tended to graduate from general to more particular in focus, largely dependent on interviewees' responses. Due to the semistructured nature of interviews, I, the interviewer, had the freedom to probe or prompt discussion of interesting issues that arose in vivo. Questions aimed to be open-ended and not leading in nature, however inevitably this was not always accomplished and my influence on the interview itself and potentially on the responses attained is undeniable.

My training in Clinical Psychology equipped me with counselling skills which proved useful such as reflection or paraphrasing interviewees' responses to ensure that my interpretation matched what the interviewee was intending to communicate (enhancing respondent validation), as well as rapport-building skills such as advanced empathy and unconditional positive regard. I collected field notes during and following interviews regarding my observations and impressions of participants and their responses. Interesting findings were noted, including tangential topics raised by 
interviewees. These field notes influenced topics followed up on in subsequent interviews and helped to inform the analysis.

As a quality check, after the pilot interview, I transcribed the recording immediately and distributed the transcription to my supervisory panel. I also listened to the interview several times to review my interview technique and the interview schedule. Whilst the interview was deemed satisfactory overall and data from it has been included in that anaylsis, several changes were subsequently made in my approach to interviews. As previously mentioned, interviews from thereon were one-to-one as opposed to involving two consumers at once. Moreover, the demographic information section of the interview was also recorded, as field notes indicated that crucial information was gathered at this stage but had not been recorded. Additionally, my interview technique changed slightly. I noticed that during the pilot interview I remained very close to my interview schedule and, thus, did not follow up on interesting information raised by interviewees, potentially limiting my findings. I also noticed that I did not probe interviewees enough and was quick to respond to interviewees perhaps as a result of feeling uncomfortable with silences. Although these flaws in my technique were inevitably not entirely resolved following the pilot interview, my awareness of them undoubtedly lead to an improvement in my technique, which actually continued to improve with experience and as I gained knowledge from interviewees.

All interviews were digitally, audio recorded with interviewees' consent. Interviews were then saved onto a computer and transcribed verbatim into a word processing document. Noticeable pauses (depicted as ...) and non-verbal behaviour such as laughter were included in transcripts, as were interruptions and inaudible words. More detailed transcriptions 
were not required given research questions and the analytic approach. I undertook all transcribing, as the primary investigator. Albeit timeconsuming, transcribing the interviews immersed me in the data and provided me with the opportunity to review and reflect on my interview technique. Transcribing was also a way for me to commence analysis, as I became aware of codes and categories within and across interviews and was, thus, able to follow up on these in subsequent interviews. I aimed to transcribe interviews as soon as possible following their occurrence and before further interviews were conducted. This became impractical once data collection increased. Whilst I still took notes during and post interviews and reflected on interviews after their completion, listening to them again when possible, I was aware that not having tangible transcripts of interviews meant that I could not begin formal coding, which requires line-by-line analysis. I, thus, decided to stop data collection after the completion of seven interviews in order to become up-to-date with transcribing, to review my technique and to begin formal coding.

This process was in line with one of the core principles of grounded theory, which recommends that the researcher constantly shift between data collection and data analysis in order to strengthen theory generation (Glaser \& Strauss, 1967). Yet to reach theoretical saturation, I then continued interviewing, equipped with a greater understanding of the consumer perspective and more areas identified in which to follow up. Having some coding categories in mind after analyzing early interviews, when I began interviewing again I was able to follow up on these and expand on them. I conducted the following interviews in a relatively short period of time, remaining as up-to-date with transcribing as possible, re-listening to 
interviews when I was unable to transcribe them prior to the conduct of more interviews and continuing to write field notes and memos.

\subsection{Data analysis}

\subsubsection{Coding and constant comparison}

As previously mentioned, informal coding was conducted during interviews, transcribing and throughout the research process. Field notes and memos were written at various stages also to capture observations. Constant comparison took place throughout the coding process and is described below.

Formal open coding began by hand on printed transcripts. Open coding involves identifying, naming, categorizing and describing phenomena found in text (Glaser \& Strauss, 1967). Transcripts were read thoroughly and coded line-by-line. Codes developed initially were concrete; that is, they were closely linked to the data and descriptive in nature. The properties of each code were elaborated in an attempt to describe and account for all incidences of the code in the data, while continually searching for new codes. Upon application of the same code, data were checked for consistency and that which did not fit pre-established codes were labelled as new codes. The codes identified in open coding were: insight, reflection on experiences, selfmedication, forgetfulness, route of administration, storage of medication, side effects, efficacy, therapeutic alliance, community centres and peer workers, case managers, hospitalisation experiences and stigma. Of note, the latter two codes were excluded from the final analysis presented in this thesis as they were not deemed directly relevant to adherence. 
Following open coding, extracts from transcripts that represented the different codes were grouped together in word processing documents. To represent the variation that occurred within codes, within- documents codes were later divided up into sub-codes. General code and sub-code notes were written as well as notes specifically describing extracts, first by hand and then typed into documents. To illustrate, the insight code was divided into the following sub-codes: awareness of having an illness, awareness of the risk of relapse and awareness that the illness is chronic and maintenance medication is required.

The elaboration of codes was followed by the process of axial coding, whereby codes were linked together to form categories. The categories produced by axial coding were more conceptual and less descriptive of the data. Axial coding also involved a more in-depth exploration of the properties of codes and sub-codes and the broader categories themselves. The categories developed by axial coding were consumer-related factors, medication-related factors and service-related factors and encompassed the majority of the codes developed in the open-coding phase of analysis. The codes that formed the categories were then collated into Word documents and the process of elaborating and describing categories via note taking and re-organising extracts took place. The consumer-related factors category included the insight, reflection on experiences, self-medication and forgetfulness codes. The medication-related factors category encompassed the route of medication, storage of medication, side effects and efficacy codes. The service-related factors category incorporated the therapeutic alliance, community centres and peer workers and case manager codes. As connections were made between the categories in the process of selective 
coding, a theory of medication adherence amongst people with schizophrenia began to take shape.

I presented the results of coding at all stages of the process (open, axial and selective) to supervisors, including extracts from transcriptions that support codes. There was consensus about the codes developed and only a minor concern about the labelling of one of the codes (produced in open coding) was expressed. The code, originally termed 'life impact' was eventually integrated into the side effects and efficacy codes.

The subsequent analysis chapters have been organised into categories. The consumer-related factors category is presented first, followed by medication-related factors and service-related factors. The Discussion chapter (Chapter 8) summarises the categories and makes connections between them, but as previously mentioned, a process model or theory of medication adherence was not developed as it was beyond the scope of the thesis. 


\section{Chapter 5: Consumer-related factors}

The consumer-related factors category is comprised of the insight, reflection on experiences, self-medication and forgetfulness codes. These codes could be seen to relate to consumers' cognitive processes and their thinking around their illness and medication taking experiences. Of note, insight and forgetfulness are often categorised as illness-related factors in research (i.e., Compton, 2007; Moritz et al., 2009). It is difficult to distinguish how much consumers' mental illnesses account for their level of insight and forgetfulness. It has been suggested that an individual's beliefs related to their illnesses and treatments represent preserved, pre-morbid attitudes reflective of their social and cultural values, and not necessarily attitudes distorted by psychopathology (Barnes et al., 2000). Furthermore, in the analysis presented in this chapter, I will argue that insight can be gained through experience, thus, challenging exclusively medical models of insight.

This chapter commences by presenting the insight code and associated extracts. The insight code has been organised into sub-codes reflecting different types of insight: awareness of having an illness, awareness of the risk of relapse and awareness of the chronicity of the illness and the need for ongoing treatment. The reflection on experiences code is then presented, which logically proceeds given that interviewees frequently related this to gains in insight. The reflection on experiences code has been organised into sub-codes that reflect consumers' experiences at different stages of the illness and treatment process. This is followed by a sub-code that relates to interventions that treat adherence as a learning process. The self-medication 
code is then presented, followed by the forgetfulness code, which includes sub-codes that relate to strategies to overcome forgetfulness.

\subsection{Insight}

The concept of insight is widely used in psychiatry and has particular relevance to schizophrenia as poor insight represents one of the diagnostic criteria for the illness. Several different perspectives on insight have developed in the large body of research that has been conducted. For example, insight is understood as a psychological defence mechanism in some schools of thought and a cognitive deficit in others and can be classified as current or retrospective (Amador et al., 1991). At a fundamental level, Amador et al. (1991) describe a lack of insight in schizophrenia as "a seeming lack of awareness of the deficits, the consequences of the disorder and the need for treatment" and a self-perception which is "grossly at odds with that of his (or her) community and culture" (p.114). Typically, it is understood as a multi-faceted, as opposed to a unitary, construct.

Many people with schizophrenia have been observed to ignore the deficits caused by their illness and the effect their illness has on their lives (Amador et al., 1991). This lack of awareness or insight has consistently been linked to negative attitudes towards medication and treatment nonadherence in the literature (i.e., Barnes et al., 2000; Compton, 2007; Day et al., 2005; Fenton et al., 1997; Lacro et al., 2002; Lambert et al., 2004; Moritz et al., 2009; Perkins et al., 2006;). Mitchell (2007) suggests that when considering the consumer perspective in medication adherence, it is useful to consider that consumers decide when to start, adjust or stop prescribed medication according to their perceived health needs. Indeed, it makes 
intuitive sense that consumers who believe they are ill and can benefit from treatment - that is, consumers who have insight into their condition - will be more adherent and, thus, may have better clinical outcomes than those who do not believe they are ill or perceive benefits from medication. This finding is also supported by interview data in this research. In line with Amador et al.'s (1991) conceptualization of insight as a multidimensional construct, interview data also indicate that insight operates at various levels to influence adherence. Thus, within the insight code, extracts have been divided into sub-codes to represent the different aspects of it which will be presented as follows: awareness of having an illness, awareness of the risk of relapse and awareness that the illness is chronic and maintenance medication is required.

\subsubsection{Awareness of having an illness}

The awareness of having an illness sub-code is quite self-explanatory, and refers to a consumers' level of awareness of their illness symptoms and their attributions of these. Several interviewees stated that they discontinued or refused to take their antipsychotic medications altogether in the past because they did not believe that they had a mental illness; they were in denial. In some of the extracts, illness symptoms seemed to compromise insight that one has an illness, leading to non-adherence. For example, delusional thinking in relation to the interpretation of symptoms, in particular the belief that one is having a spiritual experience, represented an obstacle to insight and adherence for several interviewees. In the following extracts, medication non-adherence is typically framed as a rational response to believing one is mentally healthy, as medication is not required to treat anything. Many of the extracts listed in this section reflect retrospective insight, as interviewees talked about past experiences of non-adherence and their reasons at the time. 
This type of insight and its influence on adherence seemed most applicable to consumers during their first episode of schizophrenia, often not wanting to accept their diagnoses.

The following extracts are examples of consumers attributing nonadherence and not wanting to take medication, respectively, to thinking that they did not have a mental illness. Both extracts are in the context of discussing the early stages of the illness, specifically, the first episode. Bill, an older interviewee, also attributes his non-adherence to advice from his prescriber at the time to discontinue medication eventually as maintenance medication was not the recommended procedure at that time. Cassie frames her adherence, in spite of resistance, as resulting from threats of rehospitalisation from her parents.

Bill, 13/02/2009

B: No, I'm normal. I thought, I'm normal, I don't need, need the medication. And plus I think the doctor did say in those days that it'll, you just have to take it for a little while, you know and you'll get better.

Cassie, 04/02/2009

C: Um, I was at home so mum was making sure I took them. I didn't want to take them.

L: Yeah.

C: Um, it was like going, if you don't take them, we'll send you back to hospital and things like that. I had lots of fights with my parents and my mum especially when I first became sick you know?

L:Yes. 
C: It was stressing her out, I was stressed out myself, you know, things like that. I've got, I had 2 other brothers but I'm the only one who's got an illness in the family, lucky.

L: Hmm, yeah. And so why do you think it was at that time that you didn't want to take it?

C: I didn't believe I had an illness.

In both of these above extracts, the interviewees talk about being in denial about having a mental illness. Medication adherence is constructed as representing admission that one is not "normal" in these early stages of the illness and, therefore, is avoided. Consumers described their perceptions of themselves in these extracts as healthy and, therefore, taking medication was constructed as unnecessary. It is possible that denial of having an illness, at this early stage, functions to protect consumers somewhat from having to deal with the reality of their illnesses and the effect that it will have on their lives. In Bill's case, he may have optimistically assumed that his illness had dissipated after a brief course of medication, perhaps partly due to his doctor's instructions and, thus, discontinued his medication. While discussing her resistance to medication however, Cassie expresses frustration that she was the only one in her family diagnosed with a mental illness, indicating a reluctance to accept her fate perhaps on the grounds of the seeming injustice of her situation and the isolation that might entail from taking medication which would signify acceptance of the diagnosis and being different from the rest of the family as a result. Regardless of the motivations (if any) for denial, the above extracts provide examples of consumers refusing, or not wanting, to take their medication because of a lack of awareness of symptoms being caused by mental illness. 
The following extract is taken from the interview with Travis, a young consumer who currently works as a peer worker and has had a wide range of experiences. Peer workers are mental health consumers who are often employed by mental health community centres to provide support services to other people with mental illnesses. Travis tended to generalize his statements about schizophrenia, perhaps due to his role which would enable him to share experiences with other consumers. He talks about the difficulties accepting that one has a mental illness upon diagnosis amongst first episode consumers, using his experiences as an example.

Travis, 19/02/2009

T: Yeah! Especially um, most people that develop a mental illness, they won't um, want to accept it straight away.

L: Yeah.

T: And they'll always ask, why me? And um, they'll always just say, I had plans for my life and whether it's-, you're older or younger you know, it's not a nice feeling. You may, you may enjoy it when you're manic a little bit but the downer way outweighs that. But um, I have to say to you, the first step is, is, is you know, there's a few different steps in the acceptance, one is like, ok, so I've got an illness but the other is to move forward and start making things happen for your life.

L: Yeah, yep. I guess you can only do that once you have found that right treatment for you.

T: Yeah and that's hard, especially being my age, like 17. My friends were out partying, doing everything that teenagers should be doing, you know 
and I was sitting at home, hearing voices, hyper-ventilating and sleeping about two hours a night so um-

Travis illustrates the difficulty of accepting that one has a mental illness by contrasting his late teenage years with those of typical, mentallyhealthy peers; "My friends were out partying, doing everything that teenagers should be doing, you know and I was sitting at home, hearing voices, hyper-ventilating and sleeping about two hours a night". Travis' contrast between him and his friends when younger also functions to highlight how mental illness can be isolating and, thus, acceptance of one's diagnosis and that one is different from their peers could be undesirable, similar to Cassie in the earlier extract. Therefore, as with Cassie, denial of having a mental illness could serve a protective function for first-episode consumers as they avoid dealing with the realities of having a mental illness. Travis indicates that only once acceptance takes place can positive actions ensue. It is implied that adherence is one of these positive actions that can follow once awareness that one has a mental illness is gained. As was the case with Bill and Cassie in earlier extracts, taking medication represented admitting to being different for Travis and was, thus, avoided in the early stages of his illness.

In addition to highlighting how denial of having a mental illness can lead to non-adherence, as previous extracts have done, the following extract also indicates that once insight that one has an illness is gained, adherence to treatment may follow.

Ryan, 26/09/2008 
$R$ : It's a positive sign if they uh, if they uh, say own up to what they're experiencing. Sometimes it's just like I was the first time, just um, deny that they have a problem and then it's kinda like, they just detain you in hospital for longer until I do realise, work out that it is a problem, then treat it with the medication.

Based on his personal experiences, Ryan states that it is a "positive sign" if consumers are able to acknowledge their mental illness but points out that, unfortunately, denial is common amongst first episode consumers, consistent with his experience. Ryan indicates that denial often leads to longer periods of detainment in hospital, which can lead the consumer to then "realise" or "work out" that their mental illness is problematic and requires treatment with medication. He does not indicate the mechanism by which this realization is gained but rather frames it as merely proceeding hospitalisation. Thus, it is unclear as to whether Ryan is suggesting that he, like other consumers, gained insight from being able to self-reflect, for example, in hospital, or whether he noticed how medication improved his symptoms. Alternatively, Ryan could be interpreted as indicating that prolonged incarceration of consumers leads them to conclude that they must be sick or that the only means of being discharged from hospital is for them to be medication adherent.

The following extract highlights how medication non-adherence and relapse can represent a vicious cycle, especially for consumers whose insight into having an illness depletes as their symptoms exacerbate. This extract provides support for lack of insight as a diagnostic criterion for schizophrenia, which may become more pronounced during symptom flareups. 
Matthew, 18/02/2009

M: Now I've got insight but when I'm unwell, I haven't.

L: Oh. I see what you mean. If you don't think you're sick I guess you're not going to take your medication either.

M: That's true, yes. You don't know you're sick. You just don't know. I just don't know if I'm ill. I've got people on the inside [peers] who know if they're getting ill so they seek help quick.

L: So it kind of reaches a point maybe, like when you get sick, you find you just can't tell what's real and what's not.

M: What's real and what's not real, yeah.

L: It would be hard, wouldn't it?

M: It is hard. I'm sick and I don't know I'm sick. My mum picks it up and then the mental health picks it up.

In the above extract, whilst Matthew states that he has retrospective insight that he has a mental illness, he indicates that during episodes, he lacks insight ("Now I've got insight but when I'm unwell, I haven't"). That is, as Matthew's symptoms worsen, so too does his awareness of his symptoms ("I'm sick and I don't know I'm sick"). It could be assumed that some consumers, like Matthew, for example, may become encompassed by their symptoms such as delusions and hallucinations which may compromise their abilities to identify such experiences as illness symptoms, which could thereby lead to non-adherence. This extract is different from previous extracts, which primarily related to first or early episode experiences of consumers who were in denial about having a mental illness as Matthew states that he loses insight when his symptoms become worse and concurs 
with the interviewer that he then stops taking his medication. Matthew indicates that whilst early intervention is possible for peers who are aware that they have schizophrenia and can recognize when their symptoms are returning, he has to wait for other people to detect signs that he is relapsing. Specifically his "mum" and his "mental health" team have been able to identify warning signs of symptom fluctuations in the past. Matthew could be interpreted to imply that insight in relation to warning signs or triggers for symptom relapse can assist with adherence or at least enhance outcomes for consumers in terms of illness stability, by highlighting that his peers who have insight seek help as needed, thus, potentially avoiding negative consequences ("I've got people on the inside who know if they're getting ill so they seek help quick.")

The following extracts provide examples of people attributing their non-adherence to their interpretations of illness symptoms as spiritual experiences. That is, rather than attributing their auditory hallucinations, for example, to mental illness, they attribute them to external sources, such that a consumer may believe that they are actually talking to God, as is the example used by Katherine. Whilst Katherine talks in general terms about spiritual experiences, Margaret describes how she used to believe the voices she was hearing were real.

Katherine, 05/02/2009

L: So could you think of any strategies, or anything that you think could be useful to encourage some of these people then to stay adherent?

$K$ : Um, it's really difficult because a lot of them don't have insight, like a lot of schizophrenics, like you said, think it's a gift. And it's not a mental illness. So it's very difficult to keep those people compliant. 
L: $M m m$.

K: Because they don't see the, like, they might think, yes they do talk to God and why should I take this medication? It keeps me away from God.

L: Mmm, that's true, especially with spiritual experiences.

K: Yeah. And a lot of it is. A lot of it is that, they do think.

Margaret, 04/02/2009

M: I mean I believed in 'em implicitly til about two to three years back when I thought, you know, this is not a gift. It's uh, an illness. And it was once I started accepting that that I got better and took my medication.

In the first extract, Katherine constructs a consumer's interpretation of their hallucinations as spiritual experiences and not as illness symptoms as a barrier to adherence ("like a lot of schizophrenics, like you said, think it's a gift. And it's not a mental illness. So it's very difficult to keep those people compliant.”). According to Katherine, this type of insight, which again involves a denial of having a mental illness, leads consumers to perceive medication as unnecessary or as interfering with their "gift" and, thus, nonadherence seems a logical choice following this reasoning ("why should I take this medication? It keeps me away from God."). Katherine frames overcoming this lack of awareness of illness symptoms as extremely “difficult”, possibly because it involves challenging consumers' subjective experiences and belief systems. In the second extract, Margaret recalls how she interpreted her symptoms as a "gift" in the past and "believed in" her hallucinations/delusions. Although not included in this extract, during her interview, Margaret stated that she refused to take her medication in the past on the grounds that she did not see the need for it nor did she want her "gift" 
interfered with. Margaret connects gaining insight and, thus, "accepting" her illness diagnosis "two to three years back", to improved outcomes and medication adherence ("I got better and took my medication"). The above interviewees both, therefore, frame a lack of awareness of the symptoms of schizophrenia, such as hallucinations, and attributions of symptoms to sources other than mental illness, as negatively influencing adherence; insight into diagnoses and "acceptance" of diagnoses are framed as integral to adherence.

\subsubsection{Awareness of the risk of relapse}

The next sub-code, entitled awareness of the risk of relapse varies from the first type of insight, as in this case, the consumer may be aware that they have an illness but they lack awareness of the nature of the illness and the mechanism of medication. That is, consumers may be unaware of the risk of relapse following medication non-adherence. In extracts presented in this sub-code, interviewees frequently attributed medication non-adherence to subjective feelings of improvement or wellness. A common justification for medication non-adherence as a result of feeling better was simply the misperception that they were cured once their symptoms were relieved by medication. It is also possible that some interviewees did not necessarily assume they were cured but did not associate subjective feelings of wellness and symptom relief with taking medication.

Interviewees often attributed their past lack of insight which led to non-adherence to inadequate professional advice in relation to the consequences of non-adherence. There was also a tendency for interviewees to normalize the process of becoming non-adherent once symptoms were relieved from medication by making comparisons to people who discontinued 
medications for physical conditions. Following relapses, many interviewees reported having learned that they were not cured when they started to feel better and that they required maintenance medication. Thus, the process of becoming non-adherent and relapsing is depicted as leading to gains in insight for consumers, positively influencing future adherence. This will be elaborated further in the reflection on experiences code (5.2).

The following extracts represent those that clearly illustrate a lack of awareness about the risk of relapse. Both interviewees attributed their nonadherence to feeling well on medication and, therefore, assuming that they no longer required it.

Ruth, 31/07/2008

R: Think, oh well I'm well now so I'll get off my medication.

Gary and Ruth, 31/07/2008

L: Oh, ok, so your symptoms came on when you stopped. Do you remember what sorts of things, like, what made you decide to stop taking your medication at that point?

G: Well....because I was feeling better, ya know, I was feeling good.

L: Yeah, and you think you don't need it maybe?

G: Yeah, I thought I didn't need it anymore.

R: I told him you need your medication but he said, no, I don't need it, ya know?

The above extracts represent typical attributions for non-adherence amongst interviewees. Fairly self-explanatory, feeling "well" or "better" is linked to failure to perceive a need for medication ("I don't need it") or 
oneself as at risk of relapse to non-adherence. Thus, it could be interpreted that Gary and Ruth, above, lacked the insight to associate illness stability with taking medication. Following a positive response to medication, they may have either assumed that the medication cured them or that their illness had passed and, therefore, felt that they no longer required it. Not dissimilar to the previous extracts, in the following extract, medication non-adherence is attributed to subjective feelings of wellness leading to the un-insightful belief that one no longer requires medication. Anna acknowledges that this is a common reason for non-adherence amongst people with schizophrenia, as well as the general community.

Anna, 18/02/2009

L: Um, so I know you've gone off your medication before then. What, at that point, I know you've said that you were taking so much of it but was there anything else that influenced you to stop taking it?

A: Yeah, coz I was feeling good. Which is probably a very common thing. L: Yeah. Well I think it's almost human nature that once you start feeling good, you think, oh I don't need to take this anymore.

A: Yeah. A bit like um, antibiotics. You start to feel good and stop taking them, you don't finish the course.

Anna normalizes non-adherence as a result of noticing improvements in symptoms amongst people with schizophrenia by stating that it is likely a "common" reason for non-adherence. Further, stopping taking medication following symptom relief is co-constructed as "normal" and, therefore, reasonable by Anna and the interviewer. The interviewer's description of this process as "human nature" and Anna's comparison of non-adherence to 
antipsychotic medication and non-adherence to antibiotics function to normalize non-adherence influenced by improvements in symptoms as something observed in the general community, not just the mentally ill (" $A$ bit like um, antibiotics. You start to feel good and stop taking them, you don't finish the course."). Thus, whilst discontinuing medication treatment for a chronic illness because the consumer is feeling better is typically categorized as reflecting a lack of insight, which is considered a hallmark symptom of schizophrenia, Anna's normalization of this reasoning as generalisable to the broader community challenges the pathology of lacking this type of insight. It is implied that rather, such reasoning, albeit irrational considering the established risks for non-adherence to both antipsychotic medication and antibiotics, could be a characteristic of "human nature".

Many interviewees expressed a present awareness of the consequences of non-adherence based on past, personal experiences of discontinuing medication and ending up relapsing and being re-hospitalised as a result, as represented in the following extracts:

Oliver, 21/08/2008

O: Yes, there was, there has been a time of like, when it, when I was on medication, risperidone, and there was one time I asked, I stopped hearing voices and all that so, I don't need it anymore, I'm fixed, I'm cured, so I went off it and then that's when I went back into hospital because of it.

Gary, 31/07/2008

G: Well, um, the only encouragement I can give is like, taking medication then go off of it, even though you're feeling good, don't go off your medication because your symptoms are bound to come back sooner or later 
and that's, that's my experience of not taking the medication coz I've done it myself.

\section{$L: M m m m$.}

G: I've gone off the medication but...that hasn't helped me at all. That's, that's put me back to square one, so I recommend you take your medication all the time.

As with previous extracts, above Oliver and Gary attribute past nonadherence to perceptions that they were "fixed" or "cured" and/or no longer required medication ("I don't need it anymore") as a result of experiencing improvements in symptoms ("I stopped hearing voices") and subjective feelings of wellness ("feeling good"). Oliver's association between his experiences of discontinuing medication and being re-hospitalised ("so I went off it and then that's when I went back into hospital because of it") and Gary's association between non-adherence and his experiences of symptom fluctuations ("don't go off your medication because your symptoms are bound to come back sooner or later and that's, that's my experience of not taking the medication") reflect retrospective insight. It could be assumed that consumers gain insight about the consequences of their illness and the requirement of medication following personal experiences of non-adherence, as will be elaborated in the reflection on experiences code presented later (5.2). Indeed, many interviewees, like Gary, who reported having gained awareness of the need for medication in order to decrease the risk of relapse, became proponents of adherence and encouraged it amongst other consumers. Such interviewees often referred back to negative experiences of going off medication to support their arguments. 
Having gained an awareness of having a mental illness and acknowledging the role of medication in reducing the risk of relapse, some consumers reported having to then come to terms with living with a chronic illness which requires medication on a regular basis, for the rest of their lives. Interviewees' typical responses to this realization can be categorized as acceptance and/or frustration. Acceptance responses were typically positively framed and extracts in this category often involved normalization of maintenance medication programs by comparisons with maintenance programs that members of the mentally-healthy population are prescribed for physical conditions. Acceptance was commonly framed by interviewees as an essential pre-cursor to adherence, especially long-term adherence. Frustration responses typically involved interviewees complaining, or reporting past complaints, about having to constantly take and monitor their medication. Although not always, frustration was often linked to nonadherence. Acceptance and frustration responses to the realization that medication adherence is a lifelong were not mutually exclusive. Interviewees frequently reported experiencing acceptance punctuated by frustration and vice versa, both exercising separate influences on adherence. The following extract represents a clear example of an acceptance response:

Ruth, 31/07/2008

\section{L: What about you Ruth, how has it impacted on your life?}

R: Oh, I feel much better. I do have to take them or else you know...get unwell, so...looks like I'll have to take them for the rest of my life.

L: And how does that feel, knowing that you might have to take something for the rest of your life?

$R:$ Oh well...it's much better than getting sick, ya know? 
The above extract reflects Ruth's acceptance of her illness and acceptance of the constant need for medication to treat her illness. When asked about how she feels about having to take medication for the rest of her life, Ruth responds that medication is preferable to relapsing, thus adopting a "lesser of two evils" discourse. It could therefore be argued that consumers' awareness of having a chronic illness which requires lifelong medication therefore does not necessarily equate to positive perceptions of medication. More commonly, medication adherence was framed by interviewees who expressed acceptance as necessary, albeit unpleasant. The following extract is in the context of a consumer who has experienced past difficulties with non-adherence. Below, Thomas describes how he feels about his illness and medication at present:

Thomas, 19/2/09

T: I understand my illness now more than I used to. I understand myself a lot better than I did years ago. Um, no I do understand myself a lot better now. I understand the need for taking it [antipsychotic medication] so I take it.

Although not explicitly an acceptance response, Thomas indicates that over the years, he has gained insight and understanding into his illness, himself and the "need" for taking medication. He does not explain how he gained understanding into his illness, himself and his medication. Nonetheless, he directly links enhanced understanding or insight, particularly in relation to the "need" for medication to his adherence ("so I take it"). The following extracts compare chronic schizophrenia to physical 
conditions, both chronic and temporary, in order to normalize having to take medication on a regular basis for an extended period of time:

Ryan, 26/09/2008

R: Uh, how do I feel about it? I realise that um, I've got a condition like any other health condition that needs to take medication to um, control the symptoms and treat the illness, so I do...But see, I've realised uh, I mean it's like a lifeline, it's like someone with diabetes has to take their medication. So Ijust look at it the same way as anyone else that has a, um, uh, health problem, just have to take them...

Brodie, 21/08/2008

B: Um, yeah, it's just like taking a Panadol or something, if you've got headache. But you've gotta take it every night of the week, which is ok. I think I have to take them for the rest of my life, yeah.

In the first extract, schizophrenia is directly constructed as the same as "any other health condition" that requires medical treatment and is later likened to a chronic physical health condition, "diabetes". The comparison between schizophrenia and diabetes facilitates Ryan's construction of antipsychotic medication as "like a lifeline" and, therefore, necessary in schizophrenia management. In the next extract, Brodie could be seen to minimize medication adherence amongst people with schizophrenia, when he initially likens taking antipsychotic medication to taking pain killers consumed to treat a headache. He acknowledges, however, that he is required to take antipsychotic medication regularly and for an extended period of time ("I think I have to take them for the rest of my life"), thus, 
departing from similarities to pain killers used to treat a headache. The interviewees' normalization of requiring medication on a lifelong basis could be seen to reflect acceptance of their illness, the necessity of ongoing medication to treat the illness and a degree of integration of the illness and medication into their lives. Indeed, Brodie evaluates taking antipsychotic medication on a nightly basis neutrally ("you've gotta take it every night of the week, which is ok").

The following extract reflects a consumer's significant insight, not just relating to the nature of schizophrenia and the need for medical treatment but to how the medication works and how the schedule can be tailored to specific circumstances. Travis is talking about how he is presently lowering his dosage of medication in collaboration with his prescriber:

Travis, 19/02/2009

T: Yeah, yeah I'm just slowly doing it, you know but um, you know, I'm very in touch now with where I am. If I start feeling a bit bad, I say whoa, I sit back and I analyse it. I won't take my tablets just yet but alright, maybe it needs to go up a bit. I always talk to my doctor about it first but um, you know.

In the above extract, Travis states that he has the self-awareness to recognize in vivo when his illness symptoms are worsening ("I'm very in touch now with where I am."). He reports an appropriate response to perceived fluctuations in symptoms, involving contacting his doctor to discuss whether his medication schedule should be tailored to his situation, enabling early intervention. The extract reflects a complex understanding of the mechanism of medication, as well as early warning signs for relapse and 
Travis' initiation of an intervention response. It almost goes beyond acceptance or awareness of the need for lifelong medication and towards integration into everyday life and an attempt to gain the most from the medication schedule. This level of insight, current insight in particular (as opposed to retrospective), was uncommon amongst interviewees. The following extract is also from the interview with Travis and, again, reflects insight beyond an awareness of the need for lifelong medication to treat schizophrenia. The extract is in the context of Travis talking about what he thinks is the best approach to lowering medication dosages.

Travis, 19/02/2009

T: Well you know, I'm not a doctor but I think that the best way to do it is to slowly reduce it and feel where you're at, you know. If you start feeling a bit panicky, just stop it there for a while and let it set in a bit and then, I'm feeling alright now. Because it doesn't always change in a day. Like with me, I just lowered my tablets, 5omg, it took me about two months just to get my panicking down, you know, so it's a lot easier for me because I'm experiencing this and I know what's going on but if you're someone who's just become ill, or even two years of being ill, you know, these things are hard to accept and that can very easily make you get annoyed and just go off the rails and chuck it altogether.

Travis states that when he first had his medication dosage decreased, his symptoms, particularly anxiety, became worse and it took him two months to adapt to the lower dosage and stabilise. Travis indicates that whilst he was able to deal with the instability for two months, other consumers, especially those who are newly diagnosed or have not been ill for 
long, may find such experiences "hard to accept" and become "annoyed". Travis suggests that a frustrated response to setbacks, such as symptom fluctuations in response to lowering medication dosages, could potentially influence consumers to become non-adherent ("chuck it altogether") and "go off the rails". Travis' personal account seems to reflect not only an acceptance of having a mental illness which requires ongoing medication, but also an acceptance of the limitations of medication. There is perhaps scope for service providers to have a role in communicating the limitations of medication to consumers upon diagnosis, such as that it may not work immediately, it may not eliminate symptoms altogether, it is not a cure and it may cause side effects. Such communication could be useful to consumers so that their expectations of medication are not too high, thus, they may be less prone to feeling let down by their medication and resigning to nonadherence.

The following extracts represent frustration responses to the realization that medication is required for the rest of consumers' lives to treat chronic illness. In the previous extract, Travis spoke about how perceptions of medication as being ineffective or as taking a long time to work can lead to non-adherence. The below extract represents a first-hand account of the experience of frustration, more specifically in relation to having to take a significant amount of medication over an extended period of time, and the impact this has on adherence.

Ross, 14/08/2008

R: Um, I got sick of taking all those tablets for so long. Got fed up with taking tablets day in day out, you know? Ever since, ever since ever since I 
was a child I always had to take medication for different problems I've had. Um, when I was diagnosed with schizophrenia when I was 16, over the years the medications I've taken an' that, it's just, you just get sick and tired of taking them...Um, but that time I just got just got sick of taking the medications all the time, you know? So fed up with it, you know? Over all the years ya know?

L: And so did you just, yeah, well fair enough, you'd been taking them since you were a kid. So you I guess, you probably wouldn't have known what it was like to be off them maybe?

$R$ : Um, really no, no.

L: Mmm, and so what did happen then? Did you gradually stop or did you go off them all together?

$R$ : I went off them, I I went off most of them, yes. Nearly all of them.

In the above extract, Ross talks about a past experience of nonadherence, which he attributes to feeling frustrated about having to take medication for so long. Ross, an older consumer who is typically adherent, emphasizes the vast amount of medication he has taken over the years ("ever since I was a child I always had to take medication for different problems I've had"). When asked, Ross states that he could not remember what it was like to be off medication. Ross frames his frustration as leading to a past rejection of his medication schedule ("you just get sick and tired of taking them...I went off them"). It is possible that Ross, following years of medication treatment, was testing whether he still required medication at this stage. Thus, whilst Ross did not lack an awareness of having a mental illness and requiring medication, his response to the realisation that he had to continue taking a significant amount of medication for the rest of his life 
was met with frustration and resignation, which lead him to stop taking his medication in spite of better knowledge.

Such extracts could provide some support for studies of post-psychotic depression (PPD), which is believed to arise from a lessening of defensive denial resulting in consumers "becoming aware of the tragic circumstances of their illness" (Amador et al., 1991, p.125). That is, some consumers who attempt to integrate information from their psychosis rather than use defensive denial, may be more prone to react depressively to new insight (Amador et al., 1991). Ross' resignation and non-adherence could have been a manifestation of a depressive response to the knowledge that he would have to take medication for the rest of his life.

Interview data overwhelmingly suggested that the various forms of insight discussed exert an influence on medication adherence. Awareness of having an illness, awareness of the consequences of the illness, and awareness that the illness is chronic and requires lifelong medication treatment could represent a continuum of insight. That is, at different stages of their illnesses and as experiences are acquired, different types of insight may become more or less relevant to consumers. In the following extract, Travis deploys a metaphor to describe the process of gaining all of the aforementioned forms of insight during the course of the illness:

Travis, 19/02/2009

T: Mental illness matures and the thing is, uh, the way I see it is, when you first get an illness and you don't accept it, it's like you're a little kid trying to fight this big adult, right and then over the years, as you get on the right medication and you accept it and you start becoming well, eventually that adult becomes the kid and you're the adult, you know, so you slowly tip the 
balance and start dominating the illness so you can start controlling it and get your life back, you know and start doing things again and feeling good about yourself, you know. Just keep going.

In the above extract, Travis constructs acceptance of mental illness and medication adherence as occurring "over the years", with experiences and as the "mental illness matures". He likens the denial and lack of acceptance which, he suggests, typically occur when consumers are first diagnosed to "a little kid trying to fight this big adult". The metaphor of a child attempting to overpower an adult could be seen to function to emphasise the lack of control that mentally ill consumers who are in denial have over their illnesses. According to Travis, as time passes, and following trials, consumers eventually find "the right medication" and experience associated symptom alleviation ("you start becoming well"), the power gradually shifts from the adult to the child ("so you slowly tip the balance"). That is, Travis could be seen to imply that the consumer gains control over their illness, which becomes substantially easier to manage ("start dominating the illness so you can start controlling it"). Travis highlights the benefits associated with consumers gaining control over their illnesses which he identifies as enhancing productivity, self-esteem and lifestyles. In summary, Travis' metaphor attributes acceptance of mental illness and recognition of the need for medication, which is gained from positive experiences with a suitable medication in particular, to medication adherence and stability.

The following extract also colourfully describes with metaphors, the progression through the continuum of insight, from denial of having an illness towards acceptance: 
George, 14/08/2008

G: Oh, to tell you the truth, sticking to your medication's hard, I'm not, don't know why. I found it hard when I first like, you know, sorta in denial, you don't really wanna believe you're sick and you don't want help. Then you just, it's like becoming religious, you've gotta let the Lord into your heart. It doesn't happen straight away, you know, you've gotta go a few times before you experience it an' that, you know what I mean?

L: Yeah, I see what you're saying, so yeah, at first it's like, so did you find at first it was more difficult, because you were like, "whatever, I'm not sick". G: Yeah, yeah, I didn't want believe in 'em. Ah, it didn't really bother me, but I just thought, you know, it's like when you, when you're young and you get harassed by the cops, like "piss off copper" you know, "oh what'd you say young fella I'll take you back with me” (laughing) “ah, I'm yours copper"! No one likes the cops you know? Especially young people (laughing).

In the above extract, George emphasizes the difficulty of being adherent, especially during the early stages of illness. Basing his summary of adherence on his own experiences, George states that initially consumers are typically "in denial" about having a mental illness, thus, will not take medication on the grounds that they believe there is no need for medication ("you don't really wanna believe you're sick and you don't want help"). George then compares acceptance of the need for medication to "letting the Lord into your heart", possibly inferring that religious belief is similar to believing in the need for medication to treat illness symptoms. George could be seen to elaborate that the process of enlightenment (or acceptance of 
adherence) is not immediate but may evolve as a result of experiences ("It doesn't happen straight away, you know, you've gotta go a few times before you experience it an' that").

George then deploys a police metaphor to explain how first episode consumers may view medication. Such a metaphor could be seen to imply that medication represents a means of social control for George. He states that initially, young people being approached by the police respond with defiance, which could be seen to represent first episode consumers' denial of their illness. George elaborates that once police threaten consumers with negative consequences, they become compliant (“ah, I'm yours copper”!), which could be seen to represent awareness of the risk or experience of relapse, or hospitalization which encourages consumers to be adherent. George's description of the process of gaining insight could be interpreted as a staged process, whereby trial and error experiences lead to gains in insight, thus leading appropriately into the next code, which related to reflection on past experiences.

\subsection{Reflection on experiences - how insight is gained}

A major code which emerged as an influence on medication adherence related to the consumers' past experiences of taking or not taking their medication. The reflection on experiences code encompasses basic learning principles, such that consumers may base adherence decisions on past experiences of adherence or non-adherence, or by making comparisons between presentations prior to and after medication treatment. Commonly, for example, interviewees attributed their adherence to learning from past, 
negative experiences of non-adherence such as relapse. Interviewees also occasionally posited that their observations of other mentally ill consumers when adherent or non-adherent influenced their own adherence. These findings could be seen to somewhat contrast quantitative research, which has reported associations between recent, past non-adherence and poorer adherence outcomes at six months follow-up (i.e., Ascher-Svanum, 2006). Similarly, another study has found that the strongest predictor of adherence at six months follow-up was good adherence in the month prior to baseline (Novick et al., 2010).

In the analysis presented below, adherence is typically constructed as a behaviour which is learned and is shaped according to individual experiences. Experiences of non-adherence are frequently framed as important learning curves for consumers to reflect on, despite associations with relapse. Individualism is stressed, as is the importance of consumers' agency in relation to adherence choices, especially in the extracts that discuss interventions. Four different types of experiences that consumers reflected on and associated with their adherence will be discussed: (1) experiences of illness prior to medication treatment; (2) experiences of consequences of non-adherence; (3) experiences of the benefits of adherence; and (4) observations of other consumers. A section on interventions is also included, as when asked about how adherence could be addressed, consumers often emphasized the benefits of reflecting on and, thus, learning from, personal experiences, therefore potentially rendering ineffective interventions which aim to prevent non-adherence or entail external force to promote adherence. 
5.2.1 Experiences of illness prior to medication treatment

Some interviewees stated that their experiences of their illness symptoms, prior to diagnosis and medication treatment, influenced their adherence. When asked about their reasons for remaining adherent, interviewees occasionally referred back to a pre-diagnosis period of time and emphasized the difficulty of experiencing symptoms of schizophrenia untreated. Below, interviewees can be seen to indicate that reflecting on early illness experiences and comparing pre-treatment experiences to experiences when treated with medication provide incentive for maintaining adherence:

Ryan, 26/09/2008

R: What I do is think back, yeah, I was like this when I was unwell: Paranoid, delusional, hallucinations, um thoughts were just all over the place, couldn't function, couldn't do anything, insane basically and like, not much (inaudible) as far as sanity goes. So uh, what I do is I look back and think how bad I was, how bad my mental health was prior to getting treatment and then getting the treatment and then looking at how I was before and how I am now.

Thomas, 19/02/2009

T: Oh it's a combination. Looking back, how difficult life had been from 1965 um, I mean, it was the same when you ask my doctor then how I presented, he'd tell you, mad. It was a shocker.

L: So it's like making that comparison between how hard it was for you without the medication and with. 
T: I mean in the last 10 years or so, even longer than that, you go back 20 years, they've been a piece of cake compared to those 15 years that it was so bad at the start. They were very, very hard.

L: Right so they were hard times that you wouldn't want to go back to.

T: No. I wouldn't be prepared to take the chance.

In the above extracts, Ryan and Thomas compare their lives before receiving effective medication treatment, in the early stages of their illnesses, with their current lives, as stable, adherent consumers. Thomas' contrasting of his former, pre-medication time of life ("difficult", "very, very hard") with his adherent years ("a piece of cake") functions to emphasise the positive impact that medication treatment and adherence have had on his life. Ryan also emphasizes how difficult his life was before medication treatment by emphasizing his inability to function and describing himself as "insane". Ryan and Thomas attribute their current adherence to learning from their experiences pre-treatment and post-treatment. Specifically, in the context of being asked what motivates him to remain adherent currently, Ryan explicitly states that he "look(s) back and think(s) how bad (he) was, how bad (his) mental health was prior to getting treatment and then getting the treatment and then looking at how (he) was before and how (he is) now". Thomas' past experiences are constructed as influencing his current adherence through the statement that he "wouldn't be prepared to take the chance" to return to a pre-medication state, implying that he does not want to become non-adherent due to the associated risk of experiencing instability of his mental health and debilitating illness symptoms that he experienced in the past. 


\subsubsection{Experiences of the consequences of non-adherence}

The most common types of experiences indicated by interviewees to influence adherence were past experiences of the consequences of nonadherence. Although not dissimilar to the idea of being influenced by premedication treatment experiences, this sub-code varies slightly from the previous one in that consumers referred to more recent, post-diagnosis experiences of non-adherence which typically followed periods of adherence and stability. Many interviewees stated that their experiences of becoming non-adherent and then relapsing provided incentive for them to remain adherent, as they had learned the association between non-adherence and symptom relapse and gained insight into the need for ongoing medication treatment. Indeed, many interviewees who described having learned from experiences of non-adherence had become advocates for adherence amongst other consumers.

In the following extracts, interviewees attribute their adherence to learning that maintenance medication is necessary for their stability from a past episode of non-adherence, whereby their symptoms flared up.

Gary, 31/07/2008

L: So, what would you say motivates you to stay on your medication then now, because you've been...I know you've had a couple of times when you've stopped, but why do you keep taking your medication now?

G: Because of my past history. Ya know, I've learned that if I go off the medication I'm only gonna get worse.

L: Yep.

G: Ya know, not better, so I might as well stay on the medication and be better all the time 
Ryan

L: And so that's what motivates you to keep going then? Is it, just the fact that it keeps you stable?

R: Well, I did like, I guess I never were a guy for medication in '94, '95 and so on, but I kept saying that when I did try Abilify, and I went off clozapine in 2004, um, I just got unwell in a quick space of time and realised that hey, you know, the illness is, it just occurred to me after nine years of being well that uh, the illness is still there, so you just need to take them.

Travis, 19/02/2009

T: Um, but I think you know, with my progress, it's been a lot of years and a lot of bad experiences that have pushed me through, you know.

L: Like you've kind of learned.

T: Yeah, yeah and over that time, you know, it does get easier. It's never nice.

In the first extract, Gary directly posits his "past history" as his reason for taking his medication, elaborating that he has learned that when he discontinues his medication, his symptoms exacerbate. Reflection on this negative experience for Gary enabled him to also learn of the relative benefits of remaining adherent ("so I might as well stay on the medication and be better all the time”). Ryan acknowledges that he was never a proponent of medication in the past. He relays, however, that an experience of nonadherence - which lead to a relapse after nine years of stability whilst adherent - led to a gain in insight about the chronicity of his mental illness and, thus, influenced his current beliefs about the need for medication 
("realised that hey, you know, the illness is, it just occurred to me after nine years of being well that uh, the illness is still there, so you just need to take them."). Consistently, Travis, a peer worker who was adherent and stable at the time of interview, attributes his "progress" to time and "a lot of bad experiences". Travis concurs that he learned from negative experiences, which "pushed him through", despite acknowledging that they were "never nice". In line with the above extracts, below Steve and Thomas explicitly state that they have learned not to stop taking their medication as a result of the experiences of the consequences of non-adherence.

The experiences described in the following extracts represent secondary consequences of symptom relapse for these interviewees; hospitalization and imprisonment:

Steve, 4/02/2009

L: Yep. And um, how is that then, the gaining weight? Is that sort of a disincentive, does that sort of make you want to stop taking it?

S: Um, nah I've stopped taking my medications in the past, I have, but as soon-, I ended up back in hospital and learned my lesson not to get off 'em.

Thomas, 19/02/2009

L: Really? So it's something you've really got to keep up with. Do you take it all the time then now?

T: Certainly, yeah. Because that was my huge mistake in my 20 s when I had my first bad episode, terrible.

L: Mmm so it's like you learned from that, those experiences of going off it. 
T: Well I would say a turning point in my life was being put in the can. After being put in jail, I knew then that if I didn't follow what the doctors said and take my pills then I would have very little life to call my own.

In the first extract, even when being asked a leading question as to whether the side effect of weight gain influences Steve's adherence, he declines and justifies his adherence in spite of this side effect by associating past non-adherence with hospitalisation. It is implied that the disadvantage of adherence - namely, weight gain - is overtaken by the negative consequences of non-adherence - specifically, hospitalization. Steve then directly reinforces his current position on adherence in spite of side effects and eludes to the trial and error process involved in adherence by stating that he "learned (his) lesson not to get off 'em" from this past experience of hospitalisation. In the second extract, Thomas explicitly constructs nonadherence early in his illness as a "huge mistake" and his experience of going to jail after a bout of non-adherence as a learning curve in his life by labelling it a "turning point". Thomas constructs his experience of being jailed as teaching him of the serious life impact that non-adherence can exert and thus influencing his present adherence. In both of the above extracts, adherence is implicitly framed as a means of avoiding the negative consequences of nonadherence that can result from relapse. In the below extract, Thomas more explicitly frames adherence as a means of avoiding risks associated with nonadherence:

Thomas, 19/02/2009 
L: So um, we've talked about your experiences then with antipsychotic medications. Um, what would be your beliefs generally about antipsychotic medications and taking them?

T: Oh well, whoa, I mean you asked me when I was in Canberra what I thought about it then... I knew it had to be. I wasn't prepared to risk that happening again.

L: Mmm so it's like you wanted to leave-, well have your life as you put it. T: Sort of. Such a long way to get back after the first time and then it's a long way to get back on the second one and then that's a catastrophe.

L: So like, it's a massive setback when you go off it basically.

T: It is, yeah.

L: It's really hard to work yourself back.

T: It takes a long time.

In the above extract, Thomas refers back to a past period of time when he was non-adherent and his illness was at its peak severity. Thomas attributes his adherence to learning from this past experience of nonadherence and associated relapse. This attribution is indicated by his response to the question about his medication beliefs, whereby he refers to his episode in Canberra and states, "I knew it (adherence) had to be. I wasn't prepared to risk that happening again" which implies that associations were made between non-adherence and relapse; adherence and stability. Thomas also elaborates to construct his experiences of the difficultly and the time it takes to return to stability post-relapse, which he describes as increasing in line with the number of relapses, as influencing his present adherence. 
The following extract is novel and challenges the way adherence is defined. Below, Ryan talks about learning from experiences of the consequences of missing doses of medication for varying amounts of time:

Ryan, 26/09/2009

L: Um, so do you always, have you always remembered to take your medication?

$R:$ Uh, most of the time. And if I notice I am missing on one day, or maybe a few days is acceptable but it's unacceptable if it's 5 days or something like that, then I notice a downturn in my mental health.

In the above extract, Ryan states that he remembers to take his medication for the most part, however occasionally misses one or several daily doses. The notion of adherence as always taking medication is challenged by Ryan, who constructs missing a few doses as "acceptable" but around five days or more as "unacceptable". He indicates that he has learned from his experiences of being non-adherent for varying periods of time how long he is able to be non-adherent without experiencing relapse. Therefore, his actions, including not addressing missed doses immediately, are influenced by past experiences of the consequences, or lack thereof, of nonadherence (or partial adherence).

\subsubsection{Experiences of the benefits of medication}

In previous sub-codes, negative past experiences of the consequences of non-adherence were associated with adherence. In this sub-code, however, positive past adherence experiences are framed as influencing future adherence. Surprisingly, the incidence of this sub-code was rare, as 
adherence was typically constructed as a means of avoiding some negative consequence, such as relapse. By contrast, in the following extracts, interviewees talk about, or recommend that other consumers think back to, how well they feel/have felt on medication. Such thoughts are framed as providing incentive to continue taking medication:

Oliver, 21/08/2008

O: Don't stop takin' it man. Just think about how well you were with them on it, and if you wanna be like that, just keep takin' it man. You know, even though that you feel like you don't need it, just take it for the hell of it! (laughing)

Travis, 19/02/2009

T: And once you experience that the tablets are working, you think, shit, I'm doing alright. You're starting to feel a bit better and you start to get into a routine. Then you learn and you wanna take your tablets because you're feeling better and better. It doesn't happen straight away.

Above, the benefit of feeling well on medication is constructed as positive reinforcement for adherence. In the context of recommending other consumers to take their medication, Oliver suggests that consumers reflect on how "well" they felt when taking medication and emphasises the association between sustained well-being and adherence ("Just think about how well you were with them on it, and if you wanna be like that, just keep takin' it man"). In the latter extract, Travis explicitly states that consumers "learn" to be adherent from experiencing the positive effects of medication on symptoms. 
Central to both accounts is the importance of consumers being able to take stock to reflect and evaluate their experiences of taking medication.

\subsubsection{Observations of other consumers}

Some interviewees constructed their experiences of observing other consumers taking or not taking their medication (more commonly the latter) as influencing their own stances on adherence. The types of observations described confer with those discussed in previous extracts, such as the consequences of non-adherence, for example. Whilst all interviewees would have had personal pre-treatment experiences or their own experiences of non-adherence to draw from, it is possible that some referred to the experiences of other consumers to distance themselves from currently undesirable adherence behaviours in the context of the interview, or made general 'they' statements about consumers to distinguish themselves. It could be argued that observing and reflecting on adherence behaviours and associated outcomes of other consumers may be useful in assisting adherence amongst consumers who lack insight into their own illness and the need for medication. In the extracts below, taken from the same interview, Brodie comments on a peer's adherence behaviour and consumers generally, based on his observations:

Brodie, 21/08/2008

B: They decide not to take it then they realise that it was not the right thing to do then they end up in hospital. That's what I don't wanna do.

Brodie, 21/08/2008 
L: Can you think of any strategies that could be useful to encourage people with schizophrenia to take their medication? Like people that maybe aren't um, taking it.

B: I'd just suggest to them to take it because otherwise you'll end up probably in a pen I suppose, in a solitary ward. Those people they have, like my ex friend Frank, he was always going in and out of hospital all the time, he spent New Year's Eve on the inside once.

L: That'd be tough.

B: Yeah, and that's what I don't wanna do, so I think it's probably wise to take it anyway.

Brodie frames his observations of other mental health consumers as influencing his adherence by talking about what he has noticed are the typical consequences of non-adherence. In the first extract, he talks generally about people making the choice to stop taking their medication, then discovering that they have made the wrong choice. Brodie could be seen to imply that consumers learn that they have made a mistake in deciding to stop taking their medication, upon relapse of symptoms - that is, when it is too late because this inevitably leads to hospitalization. In the second extract, Brodie promotes adherence, justifying this by highlighting the negative consequences of non-adherence that he has witnessed his friend experience. In both extracts, Brodie follows his discussions of his observations of other consumers with a statement iterating that he does not want his fate to follow suit ("that's what I don't wanna do"), implying that he has learned from his observations that non-adherence leads to negative consequences and, therefore, he could be plausibly seen to imply that adherence will enable him to avoid these consequences. 
The following extract is in the context of Ross being asked whether he was informed about the consequences of non-adherence from his prescriber after talking about a negative experience of non-adherence and relapse. Ross frames his adherence as influenced by his personal experiences of having an illness, as well as his observations of other consumers:

Ross, 14/08/2008

L: Mmm. Did you, were you given this sort of information by anyone when you um started taking medication?

$R$ : No, I think it's by experience, more than anything. Like I've had this illness for a long time, since I was 16, I think as I've, um as I've gotten on with, with the experience of, of becoming, you get more knowledge of it, you get wiser with it you know?

L: Yeah. I guess, because you've seen what it's like when you don't take it.

$R$ : Well you, you know, also, you experience what others are like when you go into hospital too.

L: Oh yeah?

R: Yeah, because you can see what they're like, when they're ill, you know?

L: What sorts of things have you seen?

R: Um, I've seen some that with mental illness that they become very aggressive. Um, whereas others become become very um, uh the opposite really, to aggressive.

L: Like just really passive and sort of-

R: Passive, hide in their shells sort of thing. And uh those two things are pretty well, something I've noticed in other patients when I've been in hospital 
In the above extract, whilst Ross does not directly link his observations of other consumers to adherence, he does link observations to gaining knowledge about schizophrenia and the need for medication ("you get more knowledge of it, you get wiser with it you know?). Ross refers specifically to observations of other consumers in a hospital context, where he describes symptomatic consumers as either extreme of aggressive or passive. It is implied that from such observations, he has learned the need for medication and is perhaps adherent to avoid presenting like the other consumers.

\subsubsection{Interventions that promote reflection}

This sub-code somewhat integrates all of the other sub-codes. The types of experiences mentioned earlier, however, are presented in the below extracts in response to questions about how consumers could be encouraged to take their medication, rather than about the participants' experiences themselves. As such, they are typically framed as recommendations of strategies to assist with adherence. Interviewees' suggestions largely appeared based on their personal experiences of what does and does not work. Frequently, interviewees recommended that other consumers should refer to past experiences of pre-treatment, adherence or non-adherence to assist them with choices around medication use, thus providing further evidence subjective experiences can influence adherence. In the below extracts, interviewees recommend that consumers compare how they feel when taking medication to how they feel when they are not, in order to encourage adherence:

Molly, 18/02/2009

M: If they don't take it you become uh, very sick. 
L: So if you don't take it, your symptoms will come back, you'll get sick. Um how do you think we could get this across?

M: Tell them that, that it's no good.

L: Have you found any strategies useful to encourage you to take it?

M: Well, I think of how I was when I stopped it.

L: Yes.

M: And how I am now, much better now.

L: So you make like a comparison.

M: A comparison.

Ryan, 26/09/2008

L: Is there anything you think could be done to help people with adhering to their medication at all? I know you've mentioned the comparison between, like pointing out to someone, this is what you were like before you were on it and this is how you are now.

R: Yeah but the person who takes the medication has to do their own bit of diagnosing. Say, ok this is how it is now, this is how it was then, obviously it's better now so I have to keep taking the medication.

L: So something the people have to learn themselves.

In the first extract here, Molly directly associates non-adherence with becoming ill and constructs this as consistent with her past experiences. Ryan implicitly constructs non-adherence as a negative experience by comparing the present (adherence) with the past (non-adherence) and describing the former as "better". In the context of being asked about interventions to address adherence, both interviewees indicate the usefulness of reflecting on past experiences of non-adherence and comparing these to 
those when adherent. It is implied in both extracts that making such comparisons will facilitate consumers to make the association between medication adherence and stability, and that this will, in turn, motivate them to remain adherent. Both interviewees could be seen, thereby, to indirectly frame past experiences of non-adherence as important to reinforce future adherence, as they highlight the benefits of taking medication. In the second extract, Ryan also emphasizes the subjectivity of adherence choices, by stating that "the person who takes the medication has to do their own bit of diagnosing" in response to being asked about interventions. By diagnosing, Ryan seems to be referring to a process whereby the consumer makes the decision as to whether they need medication based on an appraisal of their experiences on and off medication. Interventions from external sources are, thus, implicitly constructed as less effective by Ryan, through his representation of adherence as a personal choice, influenced by personal experiences. Similar to the previous extracts, the below extracts more directly emphasise the importance of non-adherence experiences in assisting with future adherence.

Oliver, 21/08/2008

L: And um, how do you think some of these, what could we do to get this across, do you think just tell people this, give people this sort of information?

O: Yeah, well, what you should, if they don't think they need it, you should say, alright then, don't take it and then when they're, something happens, goes wrong, use that as an example, like if they start hearing voices and that again, put 'em on their medication and wait until they're better and the next time they feel that they don't need medication just bring back the time 
when they did go off it and started falling in the dumps and all that and hearing voices and all that and bring that all up, say you do need it, this is what happens, it's happened to you in the past, so you take it.

L: Yeah.

O: Yeah so put it as a, use it as a reminder.

Thomas, 19/02/2009

T: Well look, I don't know. I had those problems myself. I had a brother who was a doctor and he'd tell me how important it was that I stayed on them and in the end I decided not to.

L: Yeah. Do you think anything could have helped you then?

T: I don't know. I think maybe you just have to learn the hard way.

In both of these extracts non-adherence experiences are constructed as important influences on future adherence, as interviewees indicate that consumers can learn the association between adherence and stability by drawing on these experiences. Like the previous extracts, it is suggested that mere instruction to take medication, even in conjunction with information about the risks of non-adherence, is ineffective in assisting with adherence. Thomas summarises this position through the statement, "I think maybe you just have to learn the hard way", framing adherence as something which is learned via a trial and error process. Thomas states that only once a consumer has experienced non-adherence and relapsed, can health workers then have a role in reminding consumers of this experience to assist with motivation for adherence. The following extract uses a metaphor to describe the learning process involved in adherence: 
Travis, 19/02/2009

T: But everyone has to, at some stage, work this out for themselves, with a mental illness. They have to work it out and they have to start learning this stuff to progress. It's just like, you're at uni, you can't expect to go to uni for 6 months and then graduate, you've gotta go through it, you know what I mean?

The above extract took place in the context of Travis talking about how consumers can be made aware of the importance of medication adherence. Travis constructs adherence as a process which is personal and involves learning from experiences ("everyone has to, at some stage, work this out for themselves, with a mental illness. They have to work it out and they have to start learning this stuff to progress"). Travis could be seen to imply that the process of becoming adherent cannot be hastened by outside intervention, but rather, is a natural, learning process which evolves with time; he uses the metaphor of university education to illustrate this. Specifically, through the metaphor of expecting an individual to graduate after a short period of time, Travis could be seen to highlight the irrationality of expecting consumers to be adherent immediately. He could additionally be interpreted to suggest that the process of learning about the need for medication is associated with experiences ("you've gotta go through it"). The following extracts strongly emphasise the subjectivity of experiences of mental illness and with medication, which contraindicate the effectiveness of general interventions:

Cassie, 04/02/2009

C: Um, no that's what the individual's gotta learn for themselves. You might be able to help them with a case manager or someone that and get 
someone to talk to them, get them to become compliant earlier, but they've gotta learn it themselves, that that's what they want.

Matthew, 18/02/2009

L: What would be some strategies then, how could we encourage people to stay on their medication then?

M: Well it's really up to them.

L: Yep, heaps of people have been saying that, like it's kind of an individual thing.

M: Yep, it's up to them.

L: So is it something you've kind of gotta learn then?

M: Learn to stay on your meds and that's it.

Ross, 14/08/2008

L: Um, can you think of any strategies that could be useful to pass on to I guess, people with schizophrenia who are having some difficulty with, you know, taking their medication, or who, who might stop or not take their medication?

R: Um, uh, um, um (five second pause) just think that take, if you don't take them, um, like in my case, uh, uh (five second pause) it's hard because every person's different, with their own medication, so it's hard to know what to really say to them you know? Coz, with schizophrenia, there are different side effects you know, affects you in different ways, so it's a bit hard to know what to say to people that have got, that have got schizophrenia and have stopped taking medication.

L: So you reckon maybe the best thing would be to sort of individualise things maybe. 
R: Yeah that way.

George, 14/08/2008

L: But if you were, based on your experiences with medication, if you were to try and like help other people or to encourage other people to take it, what sorts of things would you maybe say to them?

G: Oh, you can't say nothing because everyone's got their own way of dealing with things. All depends on if they like, you know, their schiz illness, or if they hate it, or if they're disgusted with it you know? See what I mean?

Of note, the interviewer's questioning about interventions to assist with adherence in the above extracts is value-laden, in that it is assumed that adherence is always positive and something to be strived for, whereas nonadherence is the opposite. In spite of the leading nature of questions, however, interviewees could be seen to suggest that adherence decisions are consumers' prerogative and depend largely on their personal experiences ("it's up to them", "they've gotta learn it themselves, that that's what they want"). Ross and George highlight how inter-subjectivity in terms of experiences of symptoms and side effects ("it's hard because every person's different...Coz, with schizophrenia, there are different side effects you know, affects you in different ways") and their levels of insight and perceptions of their illness ("All depends on if they like, you know, their schiz illness, or if they hate it, or if they're disgusted with it you know?), for example, will influence they types of interventions that are appropriate. Thus, it could be logically extended that in order to be effective, interventions should be tailored to individual consumers' needs. By contrast, external, generalised interventions are rendered ineffective. Matthew challenges even the 
effectiveness of any external intervention, however, stating that adherence is something that consumers "learn for themselves".

In summary, interviewees indicated that their experiences of their illness pre-treatment, of the consequences of non-adherence and of the benefits of medication, in addition to their observations of other consumers, all influence adherence. Interviewees recommended strategies that involved reflecting on past experiences to assist with adherence and stressed that adherence is a learned behaviour, dependent on individual experiences. In line with these interview data, adherence should perhaps be viewed as a personal process involving learning from trial and error and evolving with experience and over time rather than an all or nothing phenomenon. Such a conceptualization of adherence challenges the implementation of generalized interventions administered by health workers to promote adherence. Interviewees' consistent referrals to the importance of non-adherence experiences as evidence to be taken into account when making future adherence decisions additionally challenges the poor tolerance of nonadherence amongst health professionals.

\subsection{Self medication}

Self-medication traditionally refers to the consumption of a substance to compensate for illness symptoms, typically without prescriber input. That is, the practice of self-treating symptoms with antipsychotic medication or other substances as desired and, thus, failing to follow the treatment prescription. As previously mentioned, Mitchell (2007) suggests that it is useful to consider the self-medication hypothesis in relation to adherence, which states that consumers decide when to start, adjust or stop prescribed 
medication according to their perceived health needs. Although not as common a code to emerge from interview data, several interviewees spoke about tailoring their medication schedules according to fluctuations in symptoms and periods of stability; for example, by increasing and reducing their dosages respectively. It appears that decisions to adhere to or discontinue antipsychotic medication are often conducted intentionally and rationally, given the information available to the consumer and their understanding of their condition (Mitchell, 2007), thus, challenging conceptions of medication non-adherence as irrational and resistant behaviour. Additionally and consistent with previous qualitative research findings, self-medication practices may reflect examples of consumers exerting control over their treatment regimens (e.g., Carder et al., 2003; Rogers et al., 1998).

Whilst the aforementioned definition of self-medication included the absence of prescriber input, several consumers indicated that they self-medicated explicitly, in consultation with their prescribers, who were positioned as supportive of the practice. Self-medication challenges definitions of adherence as it could be argued that some consumers who self-medicate are still adherent to a degree (they take their medication on a regular basis), however, their medication-taking patterns do not adhere strictly to their prescriptions. Furthermore, it is difficult to classify consumers who selfmedicate in consultation with their prescribers as either adherent or nonadherent.

In the following extract, Oliver, who takes depot antipsychotic medication, talks about keeping a reserve of oral antipsychotic medication to take in case "anything happens": 
Oliver, 21/o8/o8

O: You know, so whereas if anything happens, I've got a little bit of risperidone oral up in the cupboard.

L: Yeah.

O: Ijust keep there so if I can't get into my injection on time, I just pop one of them and that's $2 \mathrm{mg}$, so I keep a little bit spare.

L: Yep.

O: Yeah well if something happens and I can't make it to get my injection for any reason, I don't know what, I just take a tablet. It's not as, and then I go for my injection a couple of days later.

L: Yep.

O: Yeah.

L: Ok, so you've got like a back-up.

Oliver indicates that he keeps a reserve of antipsychotic medication for use when he is unable to receive his "injection on time". According to Oliver, in such cases, he self-medicates ("Ijust pop one of them"), which he constructs as effective in maintaining his stability for "a couple of days", until he receives his depot medication. Oliver's awareness that his symptoms may exacerbate when he has not had his depot dose on time and his strategy for managing symptom fluctuations that technically entail non-adherence could be seen to reflect insight regarding his illness and the effect of medication. Oliver could be seen to imply that by self-medicating with oral antipsychotic medication in instances when he is unable to attend appointments on time, he reduces his risk of relapsing. In the following extracts, similarly, Anna and 
Cassie talk about self-medicating by taking more medication than prescribed to treat symptom fluctuations:

Anna, 18/2/09

A: With the medication and self-medication stuff, like I have self-medicated on numerous occasions. Um, because I've found that the dosages, like for example with my anxiety medication, um, one wasn't enough.

L: Ohok.

A: Like I'd wait an hour or two and I'd still be a wreck so I'd take another one.

L: Aha.

A: Yeah.

L: So it just wasn't enough, wasn't helping your symptoms.

A: Mmm and then by taking the second one, I'd be taking more than what I was prescribed. It's ridiculous. But I needed to do it.

\section{Cassie, 4/2/09}

C: Gradually like um, if I'm starting to hear voices now, the voice comes at night time when I'm trying to go to sleep and sometimes I'm just like, sometimes I get up and take a bit of extra medication just to knock me out so I can go to sleep and I'm fine then the next day, but if the voices seem overpowering, and I can't go to sleep, it gets worse. So usually I take, like an extra Seroquel or something to knock me out and then the next day I'm fine, but sometimes it comes on really strong and that's when I get sick.

L: ... Cool and your doctor's cool with that? 
C: Yeah well I tell her. Usually if that happens and I ring her up, I tell her I've taken a bit extra and she says, well keep taking extra for 2 or 3 weeks or something.

Anna reports self-medicating by doubling her dosage of "anxiety" medication when she experiences an inadequate response to her medication ("I'd wait an hour or two and I'd still be a wreck"). Anna justifies the practice, by stating that "one [tablet] wasn't enough" to treat her symptoms, so she would "take another one" which she constructs as necessary ("I needed to do it"). She could be seen to challenge the rigidity of prescriptions and poor tolerance of non-adherence ("It's ridiculous"), particularly in instances when the medication dosage is insufficient to effectively treat symptoms. Cassie indicates that when auditory hallucinations interrupt her sleep (“if I’m starting to hear voices now, the voice comes at night time when I'm trying to go to sleep"), this "sometimes" prompts her to "take a bit of extra medication". According to Cassie, taking extra medication sedates her and thereby facilitates her to "go to sleep", which, she could be seen to imply, prevents relapse in some instances ("then the next day, I'm fine"). Both accounts of consumers taking more medication to treat symptom fluctuations represent examples of consumers tailoring their treatment to their individual circumstances, relying on their own judgment and, thereby, exerting control over their illness and symptom management. Cassie states that she informs her prescriber when she has self-medicated ("I ring her up, I tell her") and, therefore, she could be seen to self-medicate in collaboration with her prescriber.

Other interviewees also discussed periods of self-medication with nonprescribed drugs in order to alleviate symptoms. This practice appeared to be 
more prevalent in instances when consumers experienced significant symptoms that were inadequately treated by medication, or when they considered the effects of other substances as more tolerable than the side effects associated with medication. Consistently, Voruganti, Heslegrave and Awad (1997) suggest that consumers may adjust their medication and illicit drug use against positive or negative symptoms, side effects, or a subjective experience of dysphoria. The self-medication hypothesis would predict then, that substance misuse may be less likely if primary positive and negative symptoms are well controlled, with a minimum of adverse effects (Mueser \& Lewis, 2000). Shean (2004) suggests that schizophrenia consumers with comorbid substance abuse difficulties are more likely to be non-adherent with medication and all other treatment recommendations, partly because problems with intoxication and substance use interfere with relationships with service providers and exacerbate the effects of mental illness. It has also been proposed that consumers who use illicit substances may reject convention, be more disorganised generally, or seek to combat the stigma of mental illness by adopting the label of 'substance abuser' (Weiden et al., 1994).

In the following extracts, self-medication with non-prescribed substances is discussed:

Katherine, 5/2/09

K: And I think too that they drink because they like getting wiped out. They um, need, it, I think. With some people too, it stops their voices.

L: Yeah, it's like self-medication.

K: They self-medicate, yeah, that's right. 
Diana, 11/2/09

D: But I know other people go on their drugs because they think, we'll see, they go off the medication and they go on drugs because they're, they reckon they feel better on drugs than they do on medication.

In the above extracts, Diana and Katherine provide explanations for the relationship between schizophrenia and drug and alcohol use. Katherine suggests that in some instances, consumers "drink" because this "wipe[s them] out" and "stops their voices". Diana suggests that consumers become non-adherent ("they go off the medication") and "go on drugs" then continue to use drugs because they make them "feel better" than medication does. Diana could be referring to medication's greater propensity to induce side effects than non-prescription drugs through the comparison. Thus, consistent with the literature, both interviewees attribute self-medication with drugs and alcohol to the function of these substances to mask symptoms and their association with subjective feelings of wellness.

In the following extract, Travis discusses his personal experiences of self-medicating with alcohol and Methamphetamine. Prior to being prescribed clozapine, Travis was considered treatment-resistant. Treatment resistance may have explained/could have been caused by his substance use history:

Travis, 19/2/o9

T: Exactly, so basically I mean, once the paranoia, you know, then I started drinking and that and it got real bad and it got to the stage where I was really unwell and um, I started on meth and that all of a sudden, took away all my voices, all my panicking, everything and I felt great, you know? And 
I said to everyone, nope, I don't care. I would rather be known as a Meth[amphetamine] addict than live through what I used to without this shit, you know? I didn't know that it-, coz as you know when you're currently off of Meth, you feel shit.

L: And that can bring on hallucinations as well.

T: Yeah, it does, it does and the thing I did, well I didn't want to come down so I'd have more. So I'd never come down to the stage where I hadn't slept for 2 weeks and um, and the come-down then, it just sends you way out, you know, barely there.

L: Yeah, you don't want to go back there. So you were like self-medicating at that point with meth.

T: I definitely was because, to be honest with you, meth made me feel like I feel now. But I've just started the hard way now, you know, but people are so desperate to get out of the way they feel that they'll just about do anything, whether it be meth or just killing themselves, you know what I mean? It's not a nice feeling and um, so that's that but you know, um I can definitely say that meth is not a good thing, especially with mental illness. It will send a normal person crazy.

According to Travis, he previously unsuccessfully self-medicated with alcohol ("I started drinking") to treat his paranoia, however, his symptoms were heightened ("it got real bad"). Travis states that during this symptom fluctuation, he experimented with Methamphetamine, which treated his symptoms ("took away all my voices, all my panicking, everything") and left him feeling "great". Travis recalls that at the time, abusing Methamphetamine (and the stigma attached to being "known as" a drug "addict") was preferable to experiencing illness symptoms, consistent with 
previous research findings (Weiden et al., 1994). He emphasizes the desperation of consumers who are experiencing symptoms to contextualize why illicit drugs may represent an appealing option for them ('they'll just about do anything"). Travis constructs Methamphetamine as just as effective in treating his illness symptoms as his current medication ("Meth made me feel like I feel now"), to further justify his past self-medication. He indicates that he became addicted to Methamphetamine and increased the amount he used because he "didn't want to come down", which he associated with feeling "shit", symptom relapse and losing touch with reality ("it sends you way out"). Travis' account of his Methamphetamine use history could suggest that consumers may be even more vulnerable to substance addiction that other users, as the withdrawal effects may be greater as they can be associated with symptom relapse.

\subsection{Forgetfulness}

Forgetfulness is commonly posited as an explanation for nonadherence amongst people with schizophrenia. In a relatively recent survey study, Moritz et al. (2009) reported that one third of consumers attributed past non-adherence to forgetfulness. It has been proposed that forgetting to take antipsychotic medication may be a way to avoid the negative thoughts associated with the illness and the limits it imposes (Mueser \& Gingerich, 2006); however, in the extracts below, forgetfulness was largely framed as unintentional.

Forgetfulness was occasionally linked to symptom fluctuations in the extracts presented. Interviewees frequently indicated that incorporating medication taking into their daily routines assisted with adherence. For 
example, in some instances, interviewees stated that they took their medication at meal times each day. Other interviewees relied on prompting from social supports to remind them to take their medication. In the following extract, Brodie attributes his past non-adherence to forgetting doses:

Brodie, 21/08/2008

B: I've never stopped my medication. It probably happens. I think it happened when I first started taking medication. I missed a few lots.

\section{L: How come?}

B: I forgot or I'd been too busy doing things then I'd see I didn't take my last one; should I take it now?

Above, Brodie initially denies past non-adherence ("I've never stopped my medication"), then acknowledges that he "missed a few" doses of his medication when he "first started taking" it. He attributes his nonadherence during this period to forgetfulness and being "busy doing things". Indeed, most interviewees who reported unintentional non-adherence indicated that it occurred more frequently in the early stages of their illness, when they were newly diagnosed. Brodie elaborates that he was then faced with the dilemma of deciding whether or not to take his skipped dosage when the following dosage was due. In the following extract, Ryan also constructs forgetfulness, amongst other factors, as a possible "obstacle" to adherence:

Ryan, 26/09/2008

R: Um, obstacles to taking medication. Uh, I guess some people might take a lot of different medications so um, uh, at different times of the day, so I 
guess they have to be uh, more disciplined to take it when they've got to take it, maybe three or four times a day for some people, um, forgetting to take it or not having the medication all the time.

L: As in it might be inconvenient, like they might be out and not have their medication?

$R$ : But um personally as far as I'm concerned, if I go away on a short holiday, the first thing I think about is my medication because you've gotta take it. It's like a lifeline.

Ryan specifies that forgetfulness may be a particularly pertinent issue for consumers on complicated medication regimens ("I guess some people might take a lot of different medications...I guess they have to be uh, more disciplined to take it when they've got to take it"). Ryan highlights the difficulties of having to take medication at "different times of the day", "maybe three or four times a day". He states that consumers could forget to take their medication, or may not have it with them throughout the day, thereby leading to non-adherence. Indeed, in some of the extracts that follow, consumers talk about the inconvenience of having to consider their medication schedule in their day-to-day plans. However, Ryan states that medication is at the forefront of his mind when he considers holidaying, which he constructs as a necessity ("because you've gotta take it").

\subsubsection{Daily routine}

Interviewees commonly talked about the importance of forming a routine of taking medication to overcome unintentional non-adherence. Interviewees indicated that the process of integrating medication-taking into their routines took time, thus, possibly accounting for increased forgetfulness 
during earlier stages of the illness. Some interviewees also talked about disruptions to routines caused by changing medications. In the following extracts, Ross and Amy liken taking medication to daily routines including sleeping, eating and working:

Ross, 14/08/2008

L: Yep. Um, what would you say are some obstacles to taking your medication then?

$R$ : Um, to um, make a routine of taking them on a daily basis.

L: Yeah.

$R$ : Get into a routine of taking them. Um, yeah being a daily thing, um, uh I've been taking so many for so long, it's just a uh, normal routine you do. It's like getting out of bed or having breakfast, you know? It's just a routine that you have to do.

Amy, 10/02/2009

A: Exactly. It's [medication] as important as food shopping or going to work, but I'm going to a doctor's appointment. You've really gotta look at, I think the patient, to be successful in their recovery and rehabilitation has to look at it, not as I'm sick and useless and hopeless but as, oh ok, this is just one extra thing I have to do, you know, just like I go to the shops or whatever, just like I catch the bus to work or if I'm not well enough to work it's just like going to play tennis with my friends.

When asked about potential obstacles to adherence, Ross highlights the importance of forming a habit of taking medication daily ("make a routine of taking them on a daily basis"). Similarly, Amy states that in order 
for consumers "to be successful in their recovery and rehabilitation", they should view medication taking as "one extra thing" to incorporate into their day. Ross could be seen to imply that when consumers are first prescribed their medication and, thus, are not in the habit of taking medication, this can represent an obstacle to adherence. He elaborates that because he has been "taking so many" medications "for so long", taking medication has become a "normal routine you do". It could be logically extended that adherence may become easier with time for some consumers. Ross and Amy both deploy similes in their talk, likening medication to other daily routines such as, "getting out of bed", "having breakfast", going "to the shops", "catch[ing] the bus to work" and "going to play tennis", which function to normalize medication taking. Indeed, normalization and minimization of medication taking could reflect consumers' acceptance of the need for medication and integration of medication into their daily lives. In the next extract, Bill, who was previously on a different medication, attributes missing doses to changing his medication routine when he was prescribed a different medication:

Bill, 13/02/2009

L: So you've mentioned that sometimes-, did you just forget to take a pill?

B: Well the risperidone wasn't my usual pattern. See to do things, I have, I'm very uh, I'm a creature of habit.

L: Yeah so you like a routine.

B: A routine, yeah so I have to, have to do that otherwise I might not take it. I wasn't in the habit of taking one before bed, you know? 
Bill states that he was prescribed a new medication which he was required to take at different times ("the risperidone wasn't my usual pattern", "I wasn't in the habit of taking one before bed"). A self-described "creature of habit", Bill attributes changes in "pattern" or "routine" of his medication schedule to his past non-adherence and could be seen to imply that an absence of consistent routine could represent a future barrier to adherence for him ("otherwise I might not take it"). In the next extract, Margaret could be seen to highlight how changes in consumers' typical routine, such as holidaying, can interfere with their adherence:

Margaret, 04/02/2009

L: What about in terms of your lifestyle and that sort of thing?

M: Well I've always managed to um, work it in.

L: Yep.

M: Uh, it's a bit of a hassle getting all me medication before like the holidays, making sure I had all my medications to last the two weeks while the doctor is away.

L: Yep, so some practical sort of issues.

M: Practical things, yes. I could see how for some people they may not take it because of that.

Margaret states that she has "always managed to work [medication] in" to her lifestyle, thus minimizing the impact of taking medication on her life. She then acknowledges the "hassle" of having to arrange to have sufficient medication to take away on "holidays" and additionally having to take into consideration her doctor's schedule ("making sure I had all my medications...while the doctor is away"). Whilst she does not report 
personal non-adherence as a result of such "practical things", she constructs them as potential barriers to adherence for other consumers ("I could see how for some people they may not take it because of that").

\subsubsection{Social support}

Interviewees occasionally reported reliance on social supports to assist with adherence. Various studies have shown that adherence is positively influenced when a patient has a relative, carer or friend prepared to supervise medication, including studies of family-based interventions (Falloon et al., 1985; Kelly et al., 1990; Sperry, 1995; Young et al., 1986). Some studies have also indicated that adherence is negatively influenced by social isolation, living alone, social deprivation and lack of employment (Barnes et al., 2000). In the following extracts, interviewees talk about how friends, housemates, partners, family members and case workers have reminded them to take their medication, thus, enabling interviewees to overcome unintentional nonadherence, or have assisted with adherence by motivating them or providing constructive advice. In the following extracts, Anna and Steve talk about how co-residents, who also take medications, help them to overcome difficulties remembering to take their medication:

Anna, 18/02/2009

L: What are some of the barriers to adherence?

A: Remembering.

L: Do you think there's anything that could be done? Any strategies that could help you to remember it?

A: Well like I said, I try to take them the same time every day, my medication and well the other person in the house takes medication as well. 


\section{L: Ok, yep.}

A: So we virtually have them around the same time. So if she's home, she'll be, oh shit, tablets.

Steve, 04/02/2009

L: Do you always remember to take it?

S: Yeah. Friends that live with me, they help me, like they remind me as well, have you taken your tablet?

Anna represents "remembering" to take medication as a key influence on adherence, whereas Steve denies difficulties remembering to take his medication. When asked about strategies to overcome difficulties related to remembering to take medication, Anna posits that in addition to taking her medication at "the same time every day", which could be seen to reflect an attempt to establish a routine of medication taking, "the other person in the house" who also takes medication, has a similar routine ("we virtually have them around the same time"). According to Anna, her co-resident will prompt her to take her medication when she is home ("she'll be, oh shit, tablets"). Steve also states that his "friends", with whom he co-resides, "help" and "remind" him to take his medication. Consistent with this, in the next extract, Rachel indicates that her daughter reminds her to take her medication:

Rachel, 25/02/2009

R: It's part of my routine now, you know. It's just a done thing. I know nine thirty at night, medication. Eight thirty, nine o'clock in the morning, 
eight o'clock in the morning, medication. I know that. I've got myself into that routine.

L: Did it take you a while to get into that routine?

R: Or Miranda will say, before I take her to school, medication mummy. Have you taken your medication? You know, she's only six but she's very bright. She says to me every night when she goes to bed, don't forget to take your tablets, Mummy. Every night, don't forget to take your tablets, Mummy. You don't wanna get sick because then you won't be able to look after me.

Rachel starts off by highlighting how taking medication has become "part of [her] routine" and could be seen to imply that it has become an automatic process for her ("It's just a done thing"). She then continues to explain how her daughter asks her whether she has taken her medication before school (“Have you taken your medication?") and additionally prompts her to take her second dosage before she "goes to bed". According to Rachel, in addition to reminding her to remember to take her medication, her daughter points out the negative consequences associated with nonadherence ("You don't wanna get sick because then you won't be able to look after me"). Rachel's reliance on her daughter to reinforce adherence, who she concedes is "only six" albeit intelligent, could be seen to possibly reflect parentification of her daughter. Nonetheless, during Rachel's interview it became apparent that being able to care for her daughter motivated her to sustain her adherence. Thus, adherence could represent a means for her not to let down her daughter. Similarly, in the next extract, Nathan indicates that his girlfriend motivates him to remain adherent: 
Nathan, 25/02/2009

L: And um, you remember most of the time. Does your girlfriend remind you?

N: Yeah, she reminds me to take it. If it wasn't for her, I wouldn't take it.

L: Yeah? Because you'd forget?

N: I just wouldn't take it probably.

L: How come you wouldn't?

N: I dunno. Ijust couldn't be bothered. I don't know. She just always reminds me to take it. I would probably take it a few times but then I'd just forget.

When asked directly, Nathan concurs that his girlfriend, whom he lives with, "reminds [him] to take" his medication. He emphasizes the important role that his girlfriend plays in assisting with his adherence, by stating, "If it wasn't for her, I wouldn't take it". Nathan elaborates that in the absence of his girlfriend's support, he "would probably take [medication] a few times" but would then "just forget" and that he "just couldn't be bothered". The latter statement could be seen to imply that, in addition to helping him to overcome unintentional non-adherence as a result of forgetfulness, Nathan's girlfriend provides him with more motivation and gives him a reason to take his medication.

\subsection{Chapter summary}

The consumer-related factors category encompasses the codes of insight, reflections on past experiences, self-medication and forgetfulness. All of these codes relate to consumers' cognitive processes in some ways, including 
their attributions of symptom exacerbation and relief, their self-awareness (and in some cases, interpretations of the behaviours and mental health statuses of others), increased knowledge about their illness and medication partly as a result of their experiences, memory deficits and behavioural strategies to overcome deficits. Consistent with previous findings, insight was presented in interviewees' talk as a multi-faceted construct which operates at various levels. The types of insight that consumers identified were important to their medication adherence related to knowledge about their illness symptoms, its chronicity, the effect of medication on symptoms, and the rationale for maintenance medication. Findings from the present study suggest that consumers may gain insight as a result of learning from their past experiences. Indeed, when asked about strategies to improve adherence, many interviewees stated that they reflected on past experiences, from various periods in their lives, including pre-illness onset, premedication treatment and times when they became non-adherent and experienced negative repercussions as a result. Such findings could be seen to contradict research which frequently indicates that the best predictor of future adherence is past and present adherence, and has significant ramifications for potential interventions. In addition to referring to past experiences to reinforce adherence, extracts related to self-medication could be seen to suggest that with experience, consumers can also gain knowledge about their illnesses, optimum medication dosages and non-harmful durations of non-adherence. Having gained such knowledge, consumers may then start to modify their medication regimens themselves, tailoring it to their individual circumstances. Although sometimes done in collaboration with prescribers, this behaviour could represent consumers exercising control over their treatment. Occasionally, interviewees reported that they self- 
medicated with non-prescription substances in an attempt to alleviate symptoms. It was suggested that despite the established risks, selfmedication with substances may be considered preferable by some consumers as they may be more tolerable in terms of side effects. Finally, forgetfulness was also raised as an influence on adherence in the present study. Strategies to overcome forgetfulness provided by interviewees included incorporation of medication taking into consumers' daily lives and reliance on social supports for prompting. 


\section{Chapter 6: Medication-related Factors}

The medication-related factors raised in interviewees' talk related to the route of medication, where medication was stored and, occasionally, the complexity of the medication regimen. Predominantly, however, consumers talked about the side effects associated with medication and the efficacy of medication in treating symptoms. Specifically, the presence or absence of side effects, side effect severity and the effectiveness (or inefficacy) of medication in treating illness symptoms were commonly expressed as important influences on interviewees' attitudes towards medication and their choices to take, request to change, reduce dosage or discontinue use of their antipsychotic medication. It became apparent during the coding of interview data that side effects and the efficacy of medication were often considered collectively, or weighed against each other, in interviewees' talk. This is consistent with the findings from previous qualitative research (Carrick et al., 2004; Rogers et al., 1998; Sharif et al., 2003; Shoemaker \& Ramahlo de Oliveira, 2008).

Notably, consumers' evaluations of side effects as tolerable or intolerable were generally influenced by the impact side effects exerted on their daily lives. For example, consumers frequently reported non-adherence when side effects interfered with their capacity to perform certain roles, such as parenting or employment, or to engage in leisure activities. Similarly, whilst medication efficacy was occasionally evaluated concretely, in terms of its impact on symptoms, interviewees also talked about the impact that the medication had on their lives and their appearances to others. Interviewees' evaluations of their medications as effective or ineffective in addressing 
symptoms also frequently related to how medication improved, normalised or detracted from their mental health status and their lives. This is consistent with other research, such as a study by Rosa et al. (2005) which found that adherence amongst outpatients was associated with the Rating of Medication Influences scale items related to "perceived day to day benefits" of medication. Additionally, Carrick et al.'s (2004) qualitative exploration of the experience of side effects associated with taking antipsychotic medication amongst people with schizophrenia revealed that participants' evaluations of medication related to the total impact of medication on their functioning, feeling and appearance to the outside world.

Consumers commonly deployed dichotomies in their talk related to side effects and the efficacy of medication, such as sane/insane and normal/abnormal, to illustrate the drastic impact that side effects or symptom alleviation exerted on them and their lives. For example, side effects were deemed intolerable and linked to non-adherence when they were related to preventing consumers' "normal" undertakings. Additionally, when asked what motivated their adherence, consumers often referred to medication's efficacy in treating their symptoms, which was associated with perceptions of themselves as "sane" and/or their lives as "normal".

Dichotomies were used less in talk related to the influence of the inefficacy of medication on non-adherence.

The data analysis for the medication-related factors category begins with the route of administration of medication code. This is followed by the storage of medication code. These relatively short codes are followed by the side effects of medication code, which encompasses the associated impact of side effects on consumers' lives, which typically represented risk factors for non-adherence. The efficacy of medication code is then presented, which 
incorporates a sub-code encompassing extracts that relate to medication's inefficacy another sub-code that relates to weighing up of side effects and medication effectiveness code is then presented, highlighting the propensity of interviewees to consider these variables collectively in their adherencerelated talk.

\subsection{The route of administration}

Some interviewees indicated that the route of medication, specifically long-acting depot administration, assisted them to overcome some of the inconveniences associated with taking medication. Depot injections release antipsychotic medications in a consistent way, over a long period of time and are often prescribed for consumers with adherence difficulties and who are on community treatment orders, as the reduction in frequency of dosing is considered advantageous in terms of adherence. In a study assessing adherence to typical, depot antipsychotic medications, Heyscue et al. (1998) found an overall adherence rate of $96 \%$, which is well above rates reported for oral antipsychotic agents. The higher rates of adherence amongst consumers on depot medication regimens (Heyscue et al., 1998) may reflect the more intense service-provider follow-up of consumers on community treatment orders. Despite these statistics, treatment guidelines generally do not support depot antipsychotic medication as a first-line treatment for consumers (McGorry, 1992).

A relatively high proportion (20\% equivalent to five in total) of participants in the present study were prescribed depot antipsychotic medications. This may have been because some recruitment occurred at medication clinics, where depot medications are administered. Although not a common code, consistent with the literature, some interviewees indicated 
that a depot medication route helped them to overcome the inconveniences associated with taking oral antipsychotic medications and minimised the potential for unintentional non-adherence (i.e., as a result of forgetfulness). In the following extract, Cassie compares medication in tablet form, required to be taken daily, with long-acting depot medication:

Cassie, 4/2/09

C: Um, it's annoying, I've gotta make sure sometimes um, like if I'm going, I've gotta have some in my handbag if I'm going out in case we don't come home or something like that, so I've got some, you know, if my husband drinks too much or if I drink too much and we stay there or something I've gotta have medication on me.

L: So you need to always have it with you.

C: Yep, whereas with injections, you didn't have to worry about it so much.

Above, Cassie emphasises the added responsibilities for consumers on oral, regular doses of antipsychotic medication. Specifically, she states that she is required to take her medication with her when she leaves the house in case, for example, she or her husband drinks too much" and are away from home longer than expected, thus, medication is not instantly accessible. Cassie could be seen to imply that the onus of constantly having to be mindful of medication is inconvenient through her expression of annoyance. She contrasts having to take her medication everywhere she goes with decreased "worry" associated with "injections" of antipsychotic medication. Although not specified, it could be assumed that Cassie associates depot antipsychotic medications with comparatively less "worry" because they are long-acting and, therefore, there are decreased dosages for consumers. Although Cassie 
does not associate the route of medication with adherence, the added responsibility of having access to medication all the time could feasibly account for some unintentional non-adherence or to negative perceptions of medication, which could indirectly relate to non-adherence. In the next extract, in the context of being asked directly about how adherence could be improved in consumers, Steve highlights benefits associated with long-acting depot forms of medication:

Steve, 4/2/09

L: That's good, yeah. Um, there are a lot of people who aren't adherent. What do you think could be done to help some of these people?

S: I reckon put more tablet forms in the injections.

L: Yep?

S: Like, if they had more tablets in injections, so they they'd only have to go to John St. say once a week or once a fortnight and they get a jab and then they don't have to worry about taking medication.

L: Oh, ok, yep. And so, that's longer-lasting as well.

S: Yeah it's longer lasting and they don't have to remember to take medication, it's already in their system.

Specifically, Steve posits that more medications should become available in "injections" to assist with adherence. Like in the previous extract, Steve associates depot antipsychotic medication with less "worry" than oral forms. Steve minimizes the inconvenience associated with depot antipsychotic medications by stating that consumers "only have to go to [medication clinic] once a week or once a fortnight". He constructs depot administration as having the propensity to relieve consumers of the burden of 
having to "remember to take medication", thus unintentional non-adherence as a result of forgetfulness could be overcome.

\subsection{Storage of medication}

Interviewees occasionally indicated that where they stored their medication facilitated their adherence, with a particular positive appraisal of medication packs or dosette boxes, which organise medications according to daily dosages, amongst those who used these. It must be acknowledged, however, that interviewees infrequently spontaneously talked about medication packs; when they spoke about them, it was in response to a question in the interview. Thus, associations with adherence are predominantly co-constructions.

Some interviewees indicated that when they forgot to take their medications in the past, their medication packs or dosette boxes enabled them to overcome this potential obstacle to adherence or to act promptly to address non-adherence. In the following extract, Ross, who reported adherence difficulties in the past, highlights how using dosette boxes enables him to monitor his adherence:

Ross, 14/08/o8

L: So do you find the dosettes help?

$R$ : Yeah, a lot better, yeah, they're good, yeah.

L: In what ways do they help?

$R: U m$, they sort of, it helps you remember taking them, you know?

$L: M m m$. 
R: Umm sort of as I said you get into a routine with them and they remember to take them, like if you look at the dosette you've got, say Monday you take some, you take 'em in the morning, lunch time and tea time, uh, you uh, sort of look at the dosettes and you can see whether you've taken the lunch time or the morning one or not, so then you'll know if you've taken them or you haven't taken them sorta thing.

L: That's handy then

R: Yeah, so that's, that's a lot more easier then. If you just take them out of the packet, you don't know you know? It's a lot harder that way, you know? L: Especially when you're on a few like you're on.

R: Well when I'm on so many, yeah.

When asked about the utility of dosette boxes, Ross describes them positively ("they're good") and evaluates storing medication in them as "better" and "easier" than keeping medication in its original packaging (which he describes as "harder") in terms of monitoring adherence. With prompting, Ross concurs that monitoring medication is particularly difficult for consumers like himself, who are on complex medication regimens, thus, it could be predicted that storing medication in dosette boxes may be particularly useful in such cases. He states that dosettes assist him to "remember" by facilitating the development of a medication-taking "routine". Ross also states that by checking his dosette box regularly, he becomes aware of missed dosages ("you'll know if you've taken them or you haven't taken them"). Knowledge of skipped dosages may enable consumers to intervene appropriately and potentially restore adherence. It could also raise consumers' awareness of potential symptom fluctuations and increased risk of relapse. In the extracts below, Katherine and Margaret also highlight the 
benefits of dosette boxes and medication packs enabling consumers to monitor their adherence:

Katherine, 5/2/09

L: Where do you keep your medication?

K: I keep it in a dosette actually because when I get unwell, I actually don't remember if I've taken it or not.

L: Oh interesting.

K: Yeah so I keep it in a dosette so I can see where I'm up to.

L: Ahh, that's good. So when your symptoms get worse, it's like, probably because you're paying attention to, you know, some of the stuff that you're hearing or seeing and that sort of thing, you don't really think about your medication.

K: Yeah, yeah. Coz like in November, I kept, I didn't know whether I'd taken it or not. Like I might've taken it and then I just totally forget and then I take another lot and then I wouldn't be able to wake up and I think, oh shit, like I've doubled it.

L: Yeah, which is probably just as bad as not taking it at all, I'd imagine.

K: Yeah, that's right so I keep it in a dosette so I can keep track of it like that. And then if I'm feeling really stressed, I actually write it down, that I've taken it.

Margaret, 4/2/09

M: I did, I thought, I remember, then I thought, no that that was last night. I thought, I'll just go and check my medication pack and it was still there, so I took my tablets. 
Katherine and Margaret draw on personal experiences of overmedicating and forgetting dosages of medication to illustrate the usefulness of dosette boxes and medication packs. Katherine directly attributes her decision to store medication in a dosette box to memory difficulties related to her medication taking during symptom fluctuations ("because when I get unwell, I actually don't remember if I've taken it or not.'). Katherine also recalls past difficulties monitoring her adherence in the absence of her dosette box which lead to over-medicating and sedating side effects as a result ("then I take another lot and then I wouldn't be able to wake up and I think, oh shit, I've doubled it") and contrasts this experience with being able to "keep track" of her medication. Thus, medication packs and dosette boxes may also be useful from preventing consumers from taking too much medication. Margaret recalls an incident whereby checking her medication pack supported her adherence by helping her to avoid a skipped dosage when she could not remember whether or not she had taken her medication ("I thought, I'll just go and check my medication pack and it was still there, so I took my tablets."). In addition to enabling consumers to monitor their adherence, Travis highlights in the following extract how medication packs can also enable social supports to monitor consumers' adherence:

Travis, 19/2/o9

T: Nah, I get a medication pack.

L: And who puts it in there?

T: I get it from the medication clinic

L: Oh yep. Um, do you find that helps at all?

T: Yeah it does. I mean, you know, it makes it easy but definitely it would help for someone that actually needs it, like I don't really need it but um for 
someone that can't remember if they take they're tablets and stuff like that, which happens a lot, you know, it's really good because even if their carer comes over and looks and says, well you missed this day and that day.

Above, when asked whether his medication pack is helpful, Travis concurs and, thus, medication packs are co-constructed as facilitating medication-taking ("it makes it easy"). He highlights the particular benefits of medication packs for consumers experiencing adherence difficulties as a result of forgetfulness ("for someone that can't remember if they take they're tablets...it's really good"). Unlike previous extracts, which highlighted how medication packs can facilitate consumers to monitor their own adherence, Travis states that they can also enable carers to monitor consumers' adherence. Travis could be seen to imply that having consumers' medication packs to refer to can open up conversations between carers and consumers in relation to adherence ("if their carer comes over and looks and says, well you missed this day and that day"). Such conversations may raise consumers' awareness of, and thereby support, their adherence. For example, they may be prompted to act to address missed dosages.

\subsection{Side effects}

Adverse effects of antipsychotic medications have consistently been shown to be associated with non-adherence and poorer outcomes in research (i.e., Compton, 2007; Lacro et al., 2002; Lambert et al., 2004; Moritz et al., 2009; Rogers et al., 1998; Resnick et al., 2004). Consistently, side effects were associated with non-adherence for many interviewees in the present study. When queried about their experiences with antipsychotic medication, the 
majority of interviewees alluded to their experiences of side effects at some stage. Consistent with the literature, the types and severity of side effects reported by participants varied between types of medications and between different interviewees, as did their tolerability (Barnes et al., 2000).

Studies have additionally found that side effects of antipsychotic medications are inversely associated with quality of life (Resnick et al., 2004; Ritsner et al., 2002). This was also reflected in interviewees' talk which frequently highlighted the impact of side effects on their every day functioning, lives and appearances to the outside world, as highlighted in previous qualitative research (i.e., Carrick et al., 2004).

Although the variation of side effects raised by interviewees is not captured in the extracts that will follow, those presented all link adherence decisions and negative evaluations of medication to the experience of side effects. The below extract represents a strong anti-adherence account whereby Diana talks about "fighting" against taking her medication on the grounds that she experienced intolerable side effects that she likened to additional illness "symptoms":

Diana, 11/02/2009

D: They [medication] made it [illness] really bad. They made their own symptoms as well. They made their own side effects and also um made-, when I first went to hospital I thought I'd take it and eventually it's gonna go away and they said, it won't go away straight away. So that's alright, I took it and this stuff is really horrible stuff to take, it's not like, (inaudible) or anything like that, it's just, it gives you another effect on what mental illness is already doing because the medication wasn't making me think very well, you know what I mean? I think more suicide, I think more, not going the 
right way, I couldn't-, could only make stuff for myself a little bit but I couldn't contact my kids, I found it really hard to deal with the children, to cook for them, to do the washing and everything like that and then I get, where are you? And then, I found it easier to go off the medication.

L: Yeah because you can't really live like that, can you?

D: Yeah. Not understanding what I was going through. It also, in 2003, because I went off the medication for about three months, I can understand my mental illness, do you know what I mean? Because I tried to read a bit about it, I tried to read everything about it, but I couldn't understand it. When off my medication I could understand what it was, the symptoms I was getting and what I was feeling but it was still a lot-, I was-, it was a lot freer, I was much more fast at thinking and um, maybe then I was too fast, but then I was fighting going back on tablets, because it would make me wanna hurt my children again.

Diana recalls that she agreed to take her medication in the past as she believed that it would effectively treat/cure her illness ("I thought I'd take it and eventually it's gonna go away"). She then indicates that her expectations were inconsistent with her actual experiences of taking medication. Diana emphasises how taking medication exacerbated her condition and created secondary "symptoms", as opposed to alleviating preexisting symptoms ("it gives you another effect on what mental illness is already doing"). She elaborates to describe the specific side effects that she experienced when taking medication, including cognitive deficits ("medication wasn't making me think very well"), suicidal ideation ("I think more suicide") and harmful thoughts ("make me wanna hurt my children"). Diana constructs these cognitive and thought-related side effects as impeding 
her ability to comprehend information about her illness, to function and to parent her children ("I found it really hard to deal with the children, to cook for them, to do the washing and everything like that"). Following her emphasis of the impact side effects exerted on her life and family, Diana evaluates medication as "horrible stuff to take" and directly attributes side effects to her non-adherence, which she presents as the "easier" option. She contrasts her negative adherence experiences to non-adherence experiences, by linking the latter to an absence of cognitive deficits which enabled her to process information about her illness, thus, enhancing her understanding of her illness ("When off my medication I could understand what it was"). She additionally links her positive experiences of non-adherence to resistance to taking medication ("then I was fighting going back on tablets"). In the following extracts, consumers also talk about how experiencing various side effects influenced their evaluations of medication and adherence choices. Below, consumers directly link past non-adherence to sedation and sexual dysfunction respectively:

Steve, 04/02/2009

L: So what made you stop, if you can think back to those times?

S: Well, um, I'll give you the example of the clozapine, that used to knock you out, like half an hour after you take it you'd be zonked out for a good 10 hours.

L: Geez.

S: Yeah, so that's the reason why I stopped taking that. 
Matthew, 18/02/2009

L: Yep and I know you mentioned earlier that there was a stage when you weren't taking it.

M: That was olanzapine.

L: Yep.

M: No, no sorry, that was risperidone.

L: Was that because it wasn't working properly?

M: No. Olanzapine wasn't working for me but risperidone had sexual, something sexual, yeah.

L: Oh sexual dysfunction with it?

M: Just something sexual, yes.

L Yes. I know I have heard that that is a side effect for some people. That would be a bit of a disincentive for you then.

M: That's why I stopped taking it and I ended up in hospital in '96, yeah.

Above, Steve recalls past experiences of sedating side effects when taking clozapine ("you'd be zonked out for a good 1o hours") and attributes this to his non-adherence by directly representing sedation as "the reason why I stopped taking that". When asked about his experiences of past nonadherence, Matthew recalls experiencing "something sexual" when taking risperidone which was also directly linked to non-adherence ("That's why I stopped taking it"). Following a leading question, Matthew denies that he stopped taking risperidone due to ineffectiveness in treating his symptoms. He, thus, could be seen to imply that the sexual side effect impacted more on his evaluation of risperidone than did its primary mechanism: to treat his illness. 
In the former extracts, direct linkages were made between side effects and non-adherence. Whilst the following extracts also highlight the association between side effects and non-adherence, greater emphasis is placed on how side effects detract from the lives of consumers, or hinder them from pursuing "normal" lives, thus, influencing non-adherence. For example, below, Katherine directly links non-adherence to the negative impact of medication on consumers' lives:

Katherine, 05/02/2009

K: Yeah, they really do make you sedated. So they impact on your life. I can understand why the kids go off them because it impacts on your life.

Above, Katherine explicitly generalises that the impact of sedating side effects on young consumers' lives influences non-adherence. She could also be seen to empathise with "the kids" by stating that she "can understand why" they discontinue their medication. Her specification that the negative impact of sedation, in particular, influences adherence amongst "kids", may suggest a perception that consumers adapt to, or become more apt at managing sedating side effects with time/maturity. In the next extract, Oliver associates current non-adherence to feeling more motivated in an employment setting:

Oliver, 21/08/o8

O: Oh, I haven't really taken my medication for like three days now, and it's like I get, really work. I get excited at work I get enthusiastic about it, but then I start taking my medication, my girl makes me take my medication, and then I find that I get uh, I dunno, it's weird, I start chucking sickies an' all that I can't be bothered doing anything, I don't do the housework. I don't 
even sometimes I don't even shower; my hygiene just goes down the drain, as well. Maybe it's like, I'm on too much or something.

Oliver's account could reflect the practice of self-medicating, in the sense that he tailors his medication intake to his circumstances. Oliver admits to being non-adherent for "three days" at the time of the interview. He states that since discontinuing medication, he becomes "excited" and "enthusiastic about" his work and contrasts this experience to when he takes his medication and his attendance at work becomes inconsistent ("start chucking sickies") and he "can't be bothered doing anything" more generally, including "housework" and maintaining his personal hygiene. Oliver questions whether the dosage he is prescribed causes side effects ("Maybe it's like, I'm on too much or something."), which could explain why he decreases his regimen of his own accord.

Whilst extracts relating to the negative impact of side effects have thus far primarily focused on how the individual consumer is affected, in the next extract, Diana describes the negative impact that medication has had on her family:

Diana, 11/02/2009

D: Yeah. Except for my immediate family probably didn't notice it as much. Their attitude was like my mother in law, you just take it, it doesn't matter what you're like. But the thing is your life's going down the tube and your children's lives are getting more screwed up because I couldn't have their friends over or I couldn't have-, their lives were getting more affected because they couldn't do things the way another child was because their mother was ill. It's a circle going round, a continuous circle but it didn't have to be that vicious circle. 
During her interview, Diana described a particularly negative response to medication in the past, which she believed impacted on her brain's biochemistry, creating symptoms. In the above extract, Diana highlights the lack of sense in consumers taking medications that they are not responding well to and that exert negative impacts on the lives of the consumers and, thus, indirectly also affect their families. Her rationale is that by compromising her mental health, medication inevitably impedes her life ("your life's going down the tube"), which is impacts on the lives of her children ("and your children's lives are getting more screwed up"), who in turn are also restricted from doing the 'normal' things that other children are able to, such as having "their friends over". Several consumers, like Diana, indicated that adherence to a medication with significant side effects can be more disruptive to their lives than receiving no treatment, so became nonadherent. Such data emphasize the importance of consumers finding a medication and a dosage that they respond positively to, in order for adherence to be a realistic, viable and beneficial option. In line with this, Cassie constructs her adherence as contingent on her medication not causing side effects that compromise her ability to lead a "normal life":

Cassie, 04/02/2009

C: Um, if I can live a normal life without feeling any side effects, I wanna take my medication. When I'm, like when I was at [company name], I couldn't work there long because my eyes were rolling up at two o'clock in the morning and I couldn't just go for a break at ten o'clock and have something to eat, you know? 


\section{L: Yeah.}

C: And um, um if I can live a normal life, it's not interfering with my life I'm fine with it, but when it's making my eyes roll up or something, like when the Seroquel's on a heavy dosage, I'm pushing the psychiatrist to drop it, to give me a chance and drop it. I'll say, if it's not working, I'll increase it, but give me a chance. And she has let me drop it to 200 slowly, over-, it's good discussing things with her. It's taken about a year and a half but um I've got it down to a dosage where it's not doing that to my eyes. Um, at night time when I take it I feel tired but I take them at night time anyway,just before I go to bed so that doesn't matter.

Cassie constructs the propensity of medication to "interfere" with her life through side effects as an important predictor of non-adherence. The specific side effect that she recalls experiencing was eye rolling, which she indicates woke her up during early hours of the morning and, thus, impacted on her functioning in the workplace as she "couldn't work there long" or required early breaks. Cassie's side effects, therefore, may have also impacted on her financially, given it compromised her ability to work. Whilst Cassie indicates that she was able to persuade her psychiatrist to decrease her dosage of medication to reduce the impact of side effects, it took her " $a$ year and a half' for the dosage to be adjusted enough so that side effects did not detract from her life. In the below extracts, interviewees report sustained adherence despite experiencing side effects, highlighting potential differences in side effect tolerance amongst consumers and that side effects do not always result in non-adherence:

Anna, 18/02/2009 
A: I think it's the Risperdal giving me facial twitching at night.

\section{L: Really?}

A: Yeah, so I mean I still take it, but I still get the twitches.

Travis, 19/02/2009

T: The thing is for me, I still get nausea from my tablets but I get panicky and I just keep saying, oh yeah, that's shit but I'll be over it in a minute and it does.

L: So you get nausea, did you say?

T: Yeah from the tablets, yeah and um, and it goes and I've been doing that for years now and every time it happens, it lasts probably 10 seconds less so it's got to the stage where, when I was locked up I was having it for hours and hours, hyper-ventilating and panicking, shaking and I went pale and that, you know, to now, it only lasts about 2 minutes and I just say, give us a sec guys. I sit down for a couple of minutes and then it's gone. I don't wanna keep going-, but if I keep doing that it might go away completely one day.

Above, Anna constructs facial twitching as a side effect of her medication ("I think it's the Risperdal giving me facial twitching") and Travis constructs nausea as a side effect of his medication ("I still get nausea from my tablets"). Anna represents facial twitching as a potential obstacle to adherence by pointing out that she is adherent ("I still take it") despite the experience of this side effect (“but I still get the twitches”). She could be seen to imply that the experience of twitching challenges her adherence, however, she tolerates it. Whilst Travis acknowledges that the experience of nausea is negative ("that's shit"), he suggests that he has adapted to side effects with 
time, by constructing them as debilitating and enduring in the past ("I was locked up I was having it for hours and hours, hyper-ventilating and panicking, shaking and I went pale and that"), in contrast to manageable and short-lasting at present ("now, it only lasts about 2 minutes"). Whilst the above extracts have highlighted that experiences of side effects do not necessarily lead to non-adherence in consumers, below, Bill on the other hand, highlights how the suggestion that he could have been experiencing tardive dyskinesia partly influenced his non-adherence:

Bill, 13/02/2009

B: The reason I stopped taking my Modacate, one doctor said to me, I went to a doctor in North Adelaide and she said to me, she said, oh, I said to her, look I'm stabilized but all I want is to well, all I really want is a little bit of psychotherapy, you know, a bit of support. And she said, she sowed the seed, she said, perhaps you're suffering from a bit of tardive dyskinesia. And I thought, I'm not really. And when I, when I uh, I was functioning but when I was shaving one day, I was shaving and I looked and I saw it. It's like this. Like a stroke.

L: It tenses up or something?

B: It was like a stroke type thing, like a cramp. I saw it while I was shaving and I thought, maybe she's right.

Bill indicates that he responded positively to Modacate, a typical antipsychotic medication, in the past, by positioning himself as "stabilized" and "functioning" and recalling that he requested psychotherapy as an adjunct to effective medical treatment. Despite this positive response, Bill states that he was influenced by his doctor to discontinue taking Modacate 
due to the risk of tardive dyskinesia. Specifically, Bill details that his doctor "sowed the seed" that he could be experiencing tardive dyskinesia, following which, his awareness of side effects linked to the condition was raised and he noticed a facial cramp while shaving. Bill indicates that he did not experience side effects prior to the discussion with his doctor ("And I thought, I'm not really"), highlighting how the mere threat of side effects (and not necessarily the experience of any) can influence a consumer's experiences of taking medication and, thereby, their adherence choices.

Some consumers, like Margaret below, are prescribed other medications to address the side effects of antipsychotic medication, which could assist with adherence:

Margaret, 04/02/2009

M: When I was first diagnosed I was at [mental health facility] and I had a very, very bad reaction. All my neck twisted round, I was on Fluphenazine. And my neck twisted around and all my teeth uh, was up here. Uh, all my-

$L:$ Oh, your gums were receding?

M: No, no, no. My lips. And oh it was awful. I looked in the mirror and I thought, oh god, nooo. Um and I was really, really bad on-, all my neck and everything.

\section{L: Tensed up?}

M: Tensed up, yeah. And then the doctor came and uh, um, he uh gave me an injection and all of a sudden I went, you know, it relaxed me, and then after that I was put on benztropine.

Margaret recalls experiencing a "very bad reaction" to medication whilst in a mental health facility. She indicates that she experienced 
muscular side effects ("my neck twisted round") which were particularly distressing for her ("I looked in the mirror and I thought, oh god, nooo"). Margaret's extract highlights how prescriber intervention can assist consumers to manage side effects. In this instance, her doctor administered an injection, which alleviated the side effects ("it relaxed me"). Rather than changing medications she recalls that from thereon, she was prescribed benztropine to manage her side effects. It could be argued that the doctor's intervention for Margaret may have prevented non-adherence.

In the following extract, Anna talks about how poly-pharmacy and the side effects of this influenced her to become non-adherent:

Anna, 18/02/2009

L: Yep. Um, and so you've only been on the risperidone then, is that right? Like since you came out of hospital, that's what they put you on.

A: Yeah they put me on another, they put me on this cocktail of drugs and when I came out. Apparently I just put myself back onto what I was on before.

$L: O h, o k$.

A: Because I didn't like what it was doing to me and then I lost the plot again (laughing).

L: What were your reasons for stopping?

A: Um, the amount and I just felt like a zombie.

L: Yeah.

A: So I went from a fistful to zilch, you know, very quickly.

L: Right. A fistful is heaps to be taking, really.

A: Mmm, that's what it seemed like. And everyone said I just looked so out of it. 
In the above extract, Anna associates her non-adherence with being prescribed a particularly complex medication regimen with sedating side effects ("I felt like a zombie"). She directly represents the sedating side effects and the sheer "amount" of medication that she was prescribed as influences on her decision to discontinue medication ("because I didn't like what it was doing to me"). It is uncertain whether Anna associates the amount of medication with more side effects, or whether she perceived the regimen as too complicated to effectively be adherent to. On a side note, Anna initially states that she stopped taking the prescribed regimen and reverted to taking "what [she] was on before", challenging definitions of adherence given that she may have been following a previous prescription. In the next extract, also from Anna's interview, she negatively evaluates the practice of consumers being medicated large amounts and/or high dosages of medication upon admission to hospital:

Anna, 18/02/2009

L: So what happens when you go to hospital?

A: It's like you're too hard to deal with. Bomb them and they won't give you a hassle.

L: Yeah. So do you think they should change that as well then? Not give people so much upon admission.

A: Yeah. That's only my perception of how I felt, you know. Um, they just give it away and just bomb you. It's not right.

L: No, it's not because you're not, I mean, it's like you're not really being treated with respect. 
A: Well you can't think so if you can't think, you're really just um, I've lost the word, you're just walking and breathing really. You're just existing, you're not functioning.

Anna challenges the practice of over-medicating consumers upon admission to hospital, as have been her experiences (“It's not right”). According to Anna, hospital staff "bomb" consumers with medication to counter any resistance ("and they won't give you a hassle") or to manage difficult behaviours ("It's like you're too hard to deal with"). She recalls experiencing significant side effects as a result of being over-medicated, including cognitive difficulties ("you can't think") and describes herself as "just existing" and "not functioning". Whilst Anna does not link these experiences to non-adherence in this extract, it could contextualise her reported non-adherence to a complex medication regimen as acknowledged in the previous extract.

In the following extract, Travis challenges whether antipsychotic medication should be the first-line of treatment for people who experience psychoses, on the grounds that it causes side effects:

Travis, 19/02/2009

T: If they've got a permanent illness and they need them [medication], then that's it, you know, but if it can be calmed down and they can push through it without them, they'll never have to worry about that, you know, but it's never that easy, you know. For me, I wish I never needed them, you know. I'm not saying they didn't save my life, because they did. But my view is it's 
a good thing but it would be lovely for newer tablets to come out with less side effects.

L: Right so it's mainly the side effects that you think they should look at.

T: Yeah and you know, it makes very motivated people just not want to get off the couch. You eat so much, you put on so much weight. When I started my tablets, I was about 7okg; now I'm 120. It just puts on so much weight.

The significance of side effects to consumers is highlighted above, through Travis's representation of medication adherence as essential only for consumers who are chronically ill and are dependent on or "need" medication. He acknowledges the struggle associated with persevering with illness symptoms in the absence of medication by pointing out that consumers will have to "push through it" but constructs life "without" medication as a better alternative to medication adherence in unspecified circumstances, potentially for first episode consumers, for example, for whom the likelihood of relapse is uncertain. Despite recognizing the benefits of medication adherence for consumers ("it is a good thing"), Travis implies that the efficacy of antipsychotic medication is somewhat offset by associated side effects ("but it would be lovely for newer tablets to come out with less side effects"). Travis specifically links medication adherence to the side effects of decreased motivation, increased appetite and weight gain, using pre and post-medication adherence comparisons to emphasise the drastic impact of side effects. Travis's stance in relation to medication adherence despite attributing medication adherence to saving his life highlights the significance of side effects to consumers. 


\subsection{Efficacy}

Antipsychotic medication primarily targets the positive symptoms of schizophrenia (Van Os \& Kapur, 2009). Research consistently indicates that the efficacy (or perceived efficacy) of medication in treating illness symptoms exerts a significant influence on adherence (i.e., Kikkert et al., 2006; Moritz et al., 2009; Perkins et al., 2006; Rogers et al., 1998). In a qualitative study, similar to the present one, for example, Rogers et al. (1998) noted that for most participants, adherence was associated with recognition of the benefits of medication (such as symptom control, and relapse and hospitalisation prevention) and/or the personal costs associated with non-adherence. The main utility of medication was seen as its ability to act directly on symptoms by stopping them or reducing them to make them more manageable.

Interview data from the present study support Rogers et al.'s (1998) findings, as interviewees frequently associated the efficacy (or inefficacy) of medication to adherence (and non-adherence), respectively. The degree to which consumers' past and present medications treated illness symptoms varied. There was also inter-consumer variability in terms of responses to the same medications. The impact of medication on symptoms was emphasised through constructions of adherence as intrinsically linked to "sanity" or "normality", which were contrasted with constructions of non-adherence as related to "insanity" or "abnormality". The following extracts, thus, also tend to emphasise how mental illness can detract from consumers' lives and how medication addresses detractions. It is noteworthy that like the "Reflection on Experiences" code, this code also emphasises that experiences with medications and adherence are interlinked.

In the following extracts, direct correlations between adherence and sanity, and non-adherence and insanity, are made: 
Ryan, 26/09/2008

$R$ : Uh, um yeah, the difference between being sane and not being sane, so that's how I'd encourage other people.

Gary and Ruth, 31/07/2008

L: Yep.....ok...um...How would you say, taking the medication impacts on your life, like what sort of...I know you've said that it kind of stabilises some of the symptoms and that, but ummm, are there any other sort of ways that it has an impact on your life, taking the medication?

G: Well...we lead a better lifestyle, ya know what I mean? Because like, being, being bad and then being good is two different sides of the fences, ya know? Yourself, ya know, like...innit?

R: Yeah.

G: Because like, with the medication, it impacts a lot 'cause we're dependent, we're not dependent on it, but... it helps keep us sane sort of thing, ya know, it helps.

L: Yep.

G: Helps to um, over-ride the symptoms that we get.

In the context of being asked how adherence could be encouraged amongst consumers, Ruth emphasises the effectiveness of medication in treating symptoms through her construction of medication adherence as "the difference" between two opposing mental states: "being sane and not being sane". Gary also constructs medication adherence as the catalyst for a shift between contrasting mental states. He deploys a fence metaphor to illustrate how, by "over-rid[ing] the symptoms", medication adherence is aligned with 
"being good" and implies that non-adherence is aligned with "being bad". Gary elaborates by attributing his maintained adherence, constructed as medication "dependence", to the capacity of medication to "keep us sane". Medication adherence is additionally linked to lifestyle improvements by Gary. Medication adherence is ascribed a significant amount of power in the above extracts, as it is essentially associated with alleviating insanity. In the following extracts, interviewees promote adherence by indicating that through its efficacy in treating symptoms, medication can normalise consumers. By contrast, non-adherence and is associated with abnormality.

George, 14/08/2008

L: Yeah. And so what are some things that would, um, influence you to stay on it?

G: Umm, bein' happy and just bein' normal. If you don't take your medication people know. You're a bit loopy, you know? Even though I'm not very like...um, I'm not really that sick. I've never been on strong medication but if I don't take my medication it ain't funny you know? I always get sick.

Ryan, 26/09/2008

$R$ : Strategies, ok. The number one strategy what I'd say to someone with schizophrenia to take their medication is that sometimes, being out of the hospital, say for the first episode, for me, for example, um, it uh, they give you medication in tablet form like I did, but they may give you injections and sure, it may be sedating, a bit tiring and lack of energy taking some of these different medications for schizophrenia but the reality is, uh, then you 
realise you will turn to normal because it treats that, I guess that chemical imbalance in your mind.

In the first extract, George explicitly describes that “bein' happy and just bein' normal" influences him to remain adherent, in contrast to being a "bit loopy" and to getting "sick" when he does not take his medication. Furthermore, he describes the effects of not taking medication as being obvious to others ("people know") and implies that the public element of displaying symptoms partly influences his adherence, possibly reflective of self-consciousness in relation to his illness and awareness of the associated stigma. A clear contrast is worked up, functioning to present medication adherence as linked with being 'normal'. In addition to George's construction of being "sick" without medication, in the second extract, Ryan also appeals to the biomedical model of mental illness through his description of medication treating "that chemical imbalance in your mind". As before, medication is constructed here as alleviating this illness or abnormality: Despite side effects ("sedating, a bit tiring and lack of energy"), through its efficacy in treating the "chemical imbalance", medication allows an individual to "turn to normal". Through Ryan's reference to these side effects, followed by his construction of medication as a normalizing agent, it is suggested that the experience of side effects does not compromise the 'normal' status of consumers despite antipsychotic medication side effects being absent from the 'normal', mentally healthy human experience.

In the following extracts, Ross and Steve associate their adherence with medication's effectiveness in reducing the risk of suicide. This is contrasted with suicidal tendencies when symptoms were left untreated by 
medication. They thereby construct medication adherence as enabling them to live:

Ross, $14 / 08 / 2008$

L: What sorts of things do you find that you um, are you able to enjoy now that you couldn't if you weren't on medication?

R: Oh, I just, I wouldn't be able to cope. I wouldn't be alive today.

L: Really?

R: Yeah. Oh yeah, for sure.

Steve, 04/02/2009

L: Mmm. Yep. Um, ok, so how do you feel about taking antipsychotic medication?

S: Um, well I feel if I didn't take it that I wouldn't be alive, I don't think. I'd commit suicide, I reckon.

L: Because the voices would take over?

S: Yeah. The voices would take over and I would act on them. And I'd most probably kill myself.

Above, Ross indicates that he would not be able to "cope" and "wouldn't be alive today" without medication. He implies that he experienced suicidal tendencies when symptomatic and untreated. Steve also states that he "wouldn't be alive" if he was non-adherent. Steve elaborates that "the voices would take over" which he would "act on" by committing "suicide". Both Ross and Steve highlight the importance of the efficacy of their medication in reducing their symptoms, particularly given that when symptomatic, they become suicidal. Whilst neither of them directly link their 
adherence with their medication's capacity to reduce the risk of suicide, both could be seen to imply that adherence is a logical choice when their negative experiences associated with non-adherence are taken into account.

In the following extracts, consumers highlight how by treating their symptoms, medication improves their lives. They construct the by-products of medication adherence, including symptom relief, cognitive, emotional and social gains, as reinforcing adherence:

Anna, 18/02/2009

L: What would be then the main benefits I guess of taking your medication then?

A: Well I seem to have um, a more meaningful life um, I'm able to socialize um, and make decisions for myself.

$L: M m m$.

A: More so the right decision rather than, anything went before; what happened, happened and yeah, never really thought of the consequences before. Whereas now, yeah, that enters my head first.

L: Um, ok, so you said that it makes your life more meaningful. Um, what did you mean by meaningful?

A: Um, whereas before I suppose I had a "couldn't give a shit" attitude.

L: Oh ok, now you have a sense of purpose or something, meaning.

A: Yeah. And have more consideration of others which I never really had before.

Rachel, 25/02/2009

R: My life has got better. My life has improved with medication.

L: In what ways? 
R: Um, I'm a better mother. I'm a better person. I'm not so angry and agitated. I mean, I get agitated when I'm unwell and that but the medication, I truly believe the medication helps keep me well.

Anna and Rachel both refer to experiences of non-adherence and contrast this with experiences of adherence to emphasise how, by treating their symptoms, medication has changed them and rendered their lives more "meaningful" or fulfilling. Anna contrasts a lack of understanding of consequences when non-adherent to improved decision-making skills when adherent. Anna also contrasts a pre-adherence "couldn't give a shit attitude" with enhanced consideration of others when adherent. Rachel explicitly links taking medication to keeping her "well", implies an association between nonadherence and being "unwell", and could be seen to construct reduced agitation and anger as signifiers of wellness and thus, medication's efficacy. Rachel emphasizes personal improvements associated with adherence by referring to herself as a "better" person and mother generally. It is implied in the extracts above that other people may also benefit from the changes the consumers attribute to adherence. For example, improvements in social skills and enhanced consideration of others may improve Anna's interactions with others and it could be logically argued that Rachel's children would benefit from her improved parenting ability.

In the following extract, Gary and Ruth talk about what leading a more 'normal' life when taking medication entails for them:

Gary and Ruth, 31/07/2008

G: Yeah, well prob-...oh, just feeling better is the main thing an'...um, being able to live, live just a normal life and stuff to some degree, isn't it? 
L: Yeah.

G: Just live a normal life, because if she didn't have her medication she'd be having her panic attacks an'

L: Yeah.

R: And all sorts of things

G: All sorts of things - an' she wouldn't be right. She'd be hospitalised all the time instead of living life at home like we are now and having a bit of a normal life.

L: Ok, so it helps you to have a normal life then

R: Yeah.

L: Whereas without it...

G: Yeah.

L: So what sort of things would that involve, like what do you mean when you say a normal life?

G: Well, avoiding hospital visits

L: Yeah.

G: Plus ...avoiding hospital visits, hey

R: Yeah.

G: An', and us....not hearing voices, uhh, not being, not being, not being agitated by things an just leading a normal life, ya know what I mean? Normal, normality of it all, just being able to get up in the day and watch a bit of TV an'-

L: Yeah, I see what you're saying.

G: Instead of having a TV an' think that you know, like, it's talking to us and things like that

L: Hmmm.

G: You know what I mean, it's normality for us. 
Gary constructs medication as enabling him and his partner to lead normal lives through its efficacy in treating symptoms. This is implied through his description of a "normal life" as entailing the absence of symptoms such as anxiety, agitation, hallucinations and delusions of reference (i.e., thinking the TV is talking to them) as well as one of the consequences of symptom fluctuations; regular hospitalizations. Gary's construction of a "normal life" appears to relate to accepted conceptions of the positive symptoms of schizophrenia as additional to consumers' usual repertoire of feelings. By highlighting how these symptoms interfere with day to day activities, such as watching television, Gary underscores the pervasive life impact of active schizophrenia symptoms and constructs medication adherence as bridging the gap between the lifestyles of the mentally ill and the mentally healthy by addressing these additional experiences. Importantly, despite the repeated references to a "normal life" and normality in this extract, these descriptions are sometimes hedged: "a normal life ... to some degree", "a bit of a normal life". These descriptions can be seen to highlight that there are measures of medication's efficacy in treating symptoms. For example, in this instance, it may not completely alleviate symptoms, accounting for the hedged constructions of a "normal life". Additionally, the distinction between being mentally ill and mentally healthy is not always clear-cut.

In the next extract, in the context of discussing what motivates him to be adherent, George directly posits that "being able to do things that other people are able to do without being limited by mental illness" influences his adherence. This quote directly reflects how some consumers framed mental 
illness as limiting or detracting from their lives and, thus, constructed medication, through its efficacy in treating symptoms, as addressing limitations associated with mental illness.

George, 14/8/08

L: What else motivates you?

G: Um, my marijuana habit. I like my marijuana. Love my cones. L: Yeah.

G: Hahahha. Umm, I like women too. I like love females. I dunno. Being able to smoke cones and have relationships with people of the opposite sex - being able to do things that other people are able to do without being limited by mental illness.

George indicates that taking medication enables him to smoke marijuana, which was a novel example. During the interview, George contextualized his statement by highlighting that when he was not taking medication, marijuana use caused him to experience illness symptoms, whereas he felt medication adherence enabled him to smoke without experiencing symptom relapse. He also indicates that medication adherence enhances his ability to engage in relationships with women.

In the following extract, Cassie recalls advice she offered to a newly diagnosed acquaintance experiencing adherence difficulties. Cassie describes how she encouraged 'Angus' to take his medication by referring to the negative consequences associated with non-adherence, including relapse and lifestyle changes, based on her own experiences:

Cassie, 04/02/2009 
C: And I told him some of the terrible things that had happened and um, how I couldn't hold down a full-time job and I was lucky I had Greg to support me, and do you think you're going to find a woman to support you, because otherwise you're going to be on the pension all your life. I said, take your medication now, because he's never had a nervous breakdown from it. I said, you've just heard a few voices, you're lucky, I said, your mum detected it. I said, um, otherwise, you've got a lot of time coming, I said, like me. I said, but I'm lucky, I've got Greg to look after me. I said, you've got no one Angus. You know, I said, what woman's gonna take you on and support you? Like, I've been lucky. I said, otherwise you're gonna go through what I've been through, and you're gonna have no one. Your mum and dad will be there at the start but they're not gonna support you all your life. I said, yeah, live on the pension and be poor and he was working at the time and he had a hot car and he's still got it. And I said, but um, you'll break down Angus. I said, there goes your car, your car payments, your job.

Above, Cassie warns Angus of the "terrible things" that she experienced as a result of her symptoms not being treated by medication and constructs a "nervous breakdown" (or relapse) as an imminent risk associated with non-adherence. Thus, rather than highlighting the benefits of the efficacy of medication as previous extracts have done she focuses on the cons of non-adherence and the presence of symptoms and how this would impact on Angus' life. Cassie specifically links non-adherence to unemployment, financial difficulties and social isolation. She constructs medication as a preventative measure for Angus, who from her description, had not experienced a major psychotic episode as yet ("you've just heard a 
few voices"). Cassie recalls highlighting how non-adherence and failure to treat his symptoms could result in Angus losing things he valued, such as his car and his job and indicates that would have limited support. According to Cassie, her discussion of how medication non-adherence could impact on Angus' life was effective in influencing Angus to become adherent. This extract emphasizes not only the extent to which adherence and symptom reduction are perceived to influence the lives of consumers, but how reference to lifestyle implications could possibly be used to encourage future adherence amongst consumers. What she fails to acknowledge, however, is that medication is not always effective in treating symptoms and, thus, adherence is not always associated with lifestyle improvements or maintenance for consumers. Furthermore, whilst Cassie framed her advice as helpful, this could be seen to challenge the notion raised overwhelmingly by interviewees in the previous chapter, that experiential learning is the most effective way of reinforcing adherence.

Below, Rachel suggests that adherence could be encouraged by highlighting its capacity to assist consumers to achieve aspirations due to the benefits of symptom relief:

Rachel, 25/02/2009

R: I do it because I know I have to. If I don't, I know I'd be in a lot of trouble. Miranda [her daughter] would be taken off me and um, I wouldn't be a very nice person. I wouldn't be a very well person.

L: Can you think of any strategies that could help other people to take their medication? 
R: Nope. I just use my daughter as my incentive, that's all I can say. If they've got something that's really important to them to focus on that and, you know what I'm trying to say?

L: Yeah maybe have goals or something to work towards.

R: Yeah. If they set a goal. That's what I did. I was determined that I was going to take my medication so I could be well enough to look after my daughter on a full-time basis.

Rachel constructs medication's efficacy in treating her symptoms as reinforcing her adherence. She contrasts her mental stability when adherent (“well enough") with her mental stability when non-adherent ("I wouldn't be a very well/nice person"). According to Rachel, medication's capacity to treat her symptoms enabled her to achieve her goal of caring for her "daughter on a full-time basis". She indicates that untreated and unwell, her daughter "would be taken" (removed by authorities). By positioning her daughter as her "incentive" to remain adherent and indicating that she feels obligated to take her medication ("because I know I have to") in order to avoid having Miranda "taken", she emphasizes how the positive, secondary effects of medication efficacy that relate to consumers' lifestyles can influence adherence. Additionally, she suggests that setting goals to work towards, relating to consumers' values, which become achievable once symptoms are addressed, may facilitate other consumers struggling with adherence to become or remain adherent.

Next, Cassie attributes medication adherence to enabling her to lead a "normal" life (note, the second reference to leading a normal life is hedged " $a$ normal life most of the time") but her account is more positively framed than extracts above. That is, rather than talking about how taking medication and 
experiencing associated symptom relief can help consumers to avoid negative consequences, Cassie talks about how medication enables her to engage in various productive endeavours:

Cassie, 04/02/2009

C: Yep, I can have a normal life, like I have a normal life most of the time. And um, like I'm doing things that a normal person can do: study and work part-time and have a relationship, run a house, but I think I'm one of the luckier ones.

L: Yeah. Some people, even when they're on medication, can't do these things

C: Yeah have too many side effects, for someone to handle a relationship with them you know? And things like that.

Cassie attributes medication adherence to enabling her to undertake study, employment, to engage in a relationship and to maintain a household due to its efficacy in treating her symptoms. She acknowledges the complexity of medication adherence however, by positioning herself as "one of the luckier ones" for responding so positively to adherence resulting in her high functionality. Prompted by the interviewer, rather than contrasting positive adherence experiences with negative non-adherence experiences, Cassie highlights how for some consumers, the side effects of medication compromise their abilities to engage in "normal" life activities such as having a relationship. Medication adherence is thus constructed as having the capacity to either normalize or detract from the "normality" of a consumer's life, by its efficacy in treating symptoms or propensity to induce side effects respectively. The below extract also links the stabilizing impact of medication 
on Ryan's mental health to enabling him to lead a "normal" life whilst acknowledging the gamble of medication:

Ryan, 26/09/2008

$R:$ I'm so fortunate that the medication has given me that mental stability so I've been able to just, basically, lead a largely normal life, but uh, I guess there'll be maybe a need, if I was to go off the current medication and try something new, um, only if uh, just respect that it might fail to work for me. But I've been stable for the last, hmm, since 2000.

Ryan constructs his medication as efficacious ("medication has given me that mental stability"), which he directly links to enabling him to lead a "largely normal life". A "normal life" is evaluated positively through Ryan's description of himself as "fortunate" to be able to lead one which is suggestive of a perception of the mentally healthy populations' lives as somewhat privileged. (Note again, though, the hedging here: "a largely normal life".) Like the previous extract, his acknowledgement that a different medication may not stabilize his symptoms functions to quash generalizations about the positive impact of medication on the lives of consumers. That is, although it was common for participants to talk about the benefits of medication adherence in a general sense, in terms of symptom reduction and the opportunities this affords consumers, Cassie and Ryan's accounts highlight the complexity of medication adherence, raising the importance of the suitability of medication to the consumer. Thus, the benefits of medication adherence can arguably be seen as somewhat tenuous, with medication efficacy represented as influencing the propensity for consumers to experience the positive life impact of medication. In the 
following extract, Jason contrasts his lifestyle when taking an inefficacious medication to his present lifestyle when taking an efficacious medication:

Jason, 13/02/2009

L: Um, what would be the main incentives then to take your medication?

$J$ : Well there's more of a future out there than not taking medication and that.

\section{L: Could you explain that?}

$J:$ There is more of a stable life taking medication and that than not taking it, not seeming kind of like a zombie.

L: So with the illness, like you were sitting at home. Is that what you mean?

$J$ : Yes I was. I was sleeping a lot. When I was taking the other medication I wasn't going to school as much.

L: Oh ok, so no it's helped you to kind of lead a-

$J$ : More normal life, yeah.

At the start of the above extract, Jason attributes his medication adherence to his belief that medication affords consumers "more of a future" than non-adherence. Whilst most consumers referred to the present impact of medication adherence on their lives, several stable consumers highlighted how medication adherence had the propensity to enhance their futures and facilitate them to achieve goals. It could be argued that consumers' reluctance to talk about the how medication adherence could impact on their futures could relate to awareness that their medication may not continue to be effective, as highlighted in the previous extract. Jason draws on a dichotomy above to represent medication adherence positively, due to its 
efficaciousness by contrasting "a stable life" (when adherent) with being "like a zombie" (without medication). However, Jason also contrasts his current medication with a different medication he previously took, linking his past medication with poor school attendance. This extract, therefore, demonstrates how taking an unsuitable, inefficacious medication can have a negative impact on the lives of consumers. Indeed, he interchanges between talking about non-adherence and talking about being on "the other medication", both failing to provide symptom relief, and thereby constructed as equitable in terms of their propensities to detract from the lives of consumers.

\subsubsection{Inefficacy}

In the next series of extracts, consumers associate non-adherence with medication's inefficacy, or failure to adequately treat their symptoms. In some of the extracts presented, medication's inefficacy was also related to regularly changing medications, highlighting the important role that prescribers can have in potentially preventing non-adherence by intervening by changing medications when they are ineffective. In the following extract, Ryan attributes present adherence and past discontinuation to efficacy and inefficacy in treating illness symptoms respectively:

Ryan, 26/09/2008

R: Also, olanzapine. And I stacked the weight on.

L: Was that a reason why you went off the olanzapine?

$R$ : No, it wasn't as such. With olanzapine and risperidone, I tried them for a while between '95 and '99, but uh, it didn't treat symptoms of the illness as well as what I've got now. 
L: Yeah I have heard that clozapine is probably the best at treating symptoms, but it's not prescribed as much because of the white blood cell thing. Although that's quite rare.

R: I've heard it's quite (inaudible) antipsychotic medications, yeah. What I remember about olanzapine and risperidone, basically I found that both didn't cover um, the effects of the illness as well as what I'm taking now. risperidone, I believe from memory, still made me paranoid, being on it. olanzapine was um, weight gain and um, it covered the illness reasonably but um, nowhere near what I've currently got now.

When asked directly whether side effects influenced him to stop taking a past medication, Ryan denies this and, rather, indicates that a period of adherence "between '95 and '99" was disrupted because the medications he was taking failed to adequately alleviate his symptoms. He represents his present medication as superior in efficacy to past medications through comparisons that consistently favour his present medication ("didn't treat symptoms as well as what I've got now", "both didn't cover the effects of the illness as well as what I'm taking now", "nowhere near what I've currently got now"), thus, implying that this accounts for his present adherence. Ryan specifically talks about residual paranoia whilst taking risperidone and states that olanzapine only treated his symptoms "reasonably", suggesting that measures of effectiveness may be important for consumers in determining their adherence choices. In the next extract, Diana directly links nonadherence to inefficacy:

Diana, 11/02/2009 
C: It's so easy to go off your medication, you know what I mean? Because it doesn't work and if it doesn't work you go off it.

Diana commences the above extract by highlighting how "easy" nonadherence is for consumers, thus also implying the inverse of this; that adherence is difficult. She justifies her stance through the generalization that medication "doesn't work" which she later disclaims by premising the same statement with an "if", thus, acknowledging cases when medication is effective in treating symptoms. Diana summarises by directly attributing non-adherence to medication's inefficacy ("if it doesn't work, you go off it"), without specifying time frames or criteria for assessing effectiveness. By representing non-adherence as "easy" and linked to medication's effectiveness in treating symptoms, in conjunction with her initial generalization in relation to medication's inefficacy, she suggests that in her experiences, finding a medication that "works" is rare. In the next extract, Ross talks about changing medications frequently as a result of reductions in their effectiveness in treating his symptoms over time:

Ross, 14/08/2008

$R$ : Uh, what's happened is, with my illness, um what I've noticed with me, in my case, if I'm on any antipsychotic medication, I take it for so long and then it doesn't have an effect on it as it should. The effect of it wears off. And so then, they try another medication and see how that goes. That's what's happened with me. Each time I've taken antipsychotic medication the medication eventually wears off, and doesn't work as it should so I've had to go onto other medication, that's what seems to happened with me, it's like a pattern I have with my illness. 


\section{L: So it'll work for a while and then it}

R: It doesn't have the effect that it should've, and then I'll try another one to see if it has more better effects and that's one of the reasons why I've been taking a lot of the antipsychotic medications. Look how many medications I've been taking.

L: Yeah, you've changed quite a bit. So did you find that it stops helping your symptoms? Is that it?

R: Yeah, it stops helping your symptoms, yeah. Like you hallucinate, have delusions

L: They'll just come back after a while?

R: They just come back, yeah.

Whilst interviewees commonly indicated that it often took them several weeks to adapt to a medication and experience the full benefits of it, some interviewees, like Ross, reported that their once effective medications stopped treating their symptoms after some time. According to Ross, typically after a period of adherence and effective symptom relief, "the effect of it [medication] wears off" and, as such, he is required to trial an alternative medication. He elaborates that he experiences hallucinations and delusions when his medication "doesn't work as it should". This extract highlights the complexity of medication adherence and maintenance of adherence in particular, which could be compromised by changes to consumers' responses to medication over time. In the next extract, Ryan talks about changing medications and experiencing a relapse in his illness symptoms, which lead to hospitalization, despite remaining adherent. 
L: And what happened in 2004 then? When you were in hospital again?

R: Yeah. That's because I was changed on my medication. And my medication wasn't effective in treating the illness so I went off the medication that was treating me and went onto something new which was ineffective. It didn't work for me unfortunately. For the second time I realised, I'm not well, being paranoid and thinking things were happening which aren't.

L: And so things escalated.

$R:$ Soon enough, within a fortnight since I'd ceased to take my medication which was treating me well, I became unwell.

Ryan details above that despite his adherence to a "new" medication, because it was "ineffective" and "didn't work" for him, he experienced a relapse in illness symptoms including paranoia. The importance of the suitability of medication to the consumer is emphasized, in contrast to the generalization that all medications effectively treat symptoms; despite his adherence to medication, it still did not treat his symptoms and, thus, he relapsed. Whilst ineffectiveness was not linked to non-adherence in the above extract, it is feasible that consumers who experience relapse may be more inclined to discontinue taking their medication as they may become preoccupied with their symptoms. Below, Travis also talks about his experiences of medication not treating his illness symptoms despite maintaining adherence:

Travis, 19/02/2009 
T: Yeah well now that I sit back and analyse it, I can see exactly what happened. Um, the stage was, after meth it became-, none of the antipsychotics were working for me. None of them.

L: What other ones did you try?

T: I tried um Solian, and I was on the injection uh, Fluphenthixol or something like that. Uh, I was on Clorpromazine, um, lots of mood stabilizers, I was on sodium valproate, um I think there was a couple more. The only one I haven't tried is Seroquel.

Travis mentions above that due to his past Methamphetamine use, "none of the antipsychotics were working" for him; his illness was treatment-resistant for a period of time. He elaborates that he tried numerous antipsychotic medications, including typical and atypical medications, in addition to mood stabilizers. Travis stated during his interview that he was eventually prescribed clozapine, which treated his illness; however, other consumers with treatment-resistant schizophrenia may experience poor outcomes. Treatment-resistant consumers may represent a group for which the importance of adherence may not be able to be generalized due to the lack of associated benefits.

\subsection{Consideration of side effects and efficacy collectively}

Previous analyses have highlighted how interviewees evaluate their medications in terms of side effects and effectiveness in treating their symptoms, with references to how side effects and symptom relief associated with efficacy impacted on their lives. In the extracts that form this code, interviewees often weigh up these two variables, or consider them 
collectively, to express their views on adherence and to contextualize adherence decisions.

Illustrating the potential inter-relationships between side effects, efficacy and adherence, Lambert et al.'s (2004) study found that participants who reported side effects were more doubtful about the efficacy of antipsychotic medication in treating their illness symptoms and had significantly more negative attitudes towards medication. Negative attitudes, perceived inefficacy, and past experiences of side effects were all found to be related to non-adherence. These findings are contradicted by a recent study by Moritz et al. (2009), however, which found that participants rarely reported medication efficacy and an absence of side effects. Specifically, whilst approximately 50\% of the participants confirmed the effectiveness of antipsychotic medication, at the same time, they frequently complained about side effects.

In the extracts below, consumers frequently attributed medication adherence to experiencing a relative absence of side effects and efficacious symptom relief from their medication. Conversely, those who experienced more side effects, especially severe side effects, and whose medication was ineffective in treating their symptoms, often associated this with nonadherence. Adherence choices varied for interviewees who reported the presence of side effects in addition to some residual symptoms that their medication did not treat. The extract below demonstrates the importance of striking a balance between side effects and efficacy for consumers:

Ryan, 26/09/2008

$R$ : I've been on a few medications prior; I think about...the first medication I was put on in hospital was uh, Modacate. That was uh, covered the 
symptoms of the illness well, but left me that tired, I couldn't uh, I'd

probably only have 6 hours a day worth of activity, the rest of the time was sleep.

L: Gosh. That's a lot of sleep.

R: Yeah. That's an older medication I believe, it's not used as much at all, or much anymore. But um, it was back in 1995. So they, uh, I was on the strongest dose of that and they weaned me off it to try to match, to give me a bit more

\section{L: More energy?}

R: Energy for life, yep. But um, I've been quite, I can only have a low dose because my body's quite sensitive to it.

L: Right, you're quite sensitive to it. When you're on a higher dose you notice negative side effects, so you have to be on a lower dose.

$R$ : Yep, this dosage, the amount you take over the years and that, it's gotta be able to uh, combat the symptoms of the illness.

L: So it's like you've finally found that perfect level then, where it combats your symptoms but you're still functional.

R: Yes, for sure.

With reference to his "first" medication, Modacate, Ryan evaluates it in terms of how it "covered the symptoms" and its side effect profile. Despite constructing his medication positively with regard to symptom relief, the use of "but" preceding emphasis on the sedating side effects associated with the medication functions as a disclaimer; that is, the efficacy of the medication is constructed as being compromised by, or overshadowed by, sedating side effects. Whilst he does not attribute the side effect profile of Modacate to his non-adherence, he elaborates that his dosage was lowered as a result. Ryan 
highlights the importance of tailoring the medication and dosage to the individual consumer, by adding that when lowering the dosage to reduce side effects, it remained important that the medication can still "combat the symptoms of the illness". For many interviewees, finding the optimum dosage of medication, whereby symptoms were managed and side effects were tolerable, reportedly took a considerable amount of experimentation and time, as becomes more apparent in subsequent extracts. In the following extract, Travis talks about how the experience of side effects and of medication not treating symptoms can lead to non-adherence:

\section{Travis, 19/02/2009}

T: No one-, if someone's taking their tablets and they're getting all the side effects and none of the benefits, of course they're not gonna take the tablets. They'll chuck them straight down the sink, you know. Um, it's-, the thing is, they lose patience because the tablets take a long time to start working as well and they think, nah, I can't do this anymore, these tablets, whatever, you know and that's when things get a bit hairy and things can go way off the tracks, you know what I mean?

L: Yeah.

T: It's never, you know, it may happen one in a million but you never say, right, you've got this illness, have this tablet and you're perfect, you know. L: Yeah the next day it's kicked in and you're cured.

T: It doesn't happen like that.

L: Your body's gotta get used to it and especially then if you're trying out multiple ones.

T: Yeah and you can't-, just I know that one day, you know, you're on a tablet for a month and then you get off it for a month and then you try this 
one for a few weeks and stuff. It's a long process and people get very, very impatient with it.

Above, Travis points out that consumers who are "taking their tablets" are likely to become non-adherent ("of course they're not gonna take the tablets...they chuck them straight down the sink") if they experience "all the side effects and none of the benefits" associated with medication. He appears to be referring to the efficacy of medication in treating symptoms when he refers to the "benefits" of medication, as he later elaborates to talk about the experience of medication "working". Travis suggests that consumers may become disillusioned with their medication when it does not alleviate their symptoms promptly ("they lose patience because the tablets take a long time to start working") and become non-adherent. He thus indicates that the time that it takes for medication to start to effectively treat symptoms may additionally exert an impact on adherence. According to Travis' account, as side effects may be experienced before the medication starts to treat symptoms, this may represent a critical period for potential non-adherence amongst consumers. It could be assumed that consumers who lack awareness of the time-frames within which to expect responsiveness to medication may be particularly vulnerable to non-adherence during this period, such as newly diagnosed consumers. Travis adds that the experience of changing medications following poor responsiveness can compound negative experiences for consumers and implies that this can lead to poorer rates of adherence ("people get very, very impatient with it"). Thus, he highlights how the process of finding the right medication can be fraught with difficulties for the consumer and, therefore, challenges adherence. Below, Steve also highlights how the process of finding a suitable medication can be 
difficult for consumers, but indicates that once found, adherence facilitates consumers:

Steve, 04/02/2009

L: So um, what are some of your beliefs then about antipsychotic medication?

S: I think that they, they do help people.

L: Yep.

S: If they-, if you can find the right one that um produces the right serotonin, like increase the serotonin in your brain. If they find the right one that does that, well then yeah, it does help. As you've seen, I've been on like 3 or 4 different types of medication myself, trying to find the right one. L: Mmm. So I guess maybe that process of finding the right one is probably the hardest part, like when you're taking this stuff and you're getting side effects or-

S: Yeah, or it's not working.

L: Yeah that'd be hard.

S: That's the hardest part: Finding the right medication. But once you find it, it's easy, you know? As long as you keep on taking it, things get easier in the end.

When asked about his opinion of antipsychotic medication, Steve provides a hedged response relating to its efficacy ("I think that they, they do help people"). He constructs medication's helpfulness as contingent upon its suitability to the consumer, however ("if you can find the right one...well then yeah, it does help"). Steve agrees with the interviewer's assertion that side effects could render a medication unsuitable and adds that inefficacy 
could also ("Yeah, or it's not working"). He indicates that once a suitable medication has been identified, however, adherence enables consumers to experience the associated benefits ("As long as you keep on taking it, things get easier"). In the subsequent extract, Diana recalls her experiences of experimenting with various medications, none of which were suitable:

Diana, 11/02/2009

D: Yeah. See a lot of them didn't agree with me back then. Uh, first one, I didn't expect the side effects to be, the tablets were worse than the actual, the disease... They're not easy to take. You don't have a tablet and all your symptoms just go away. It takes a good three or four months for that to happen and then sometimes your medication doesn't work and you've gotta be open to that but, you know, our life's sort of like trying to talk to your psychiatrist about it... It wasn't until 2003. It was 2000 when I went in and it wasn't until 2003 that they actually got my medication right.

Diana evaluates the side effects of a past medication as "worse than the actual illness". She also highlights that medication does not immediately treat symptoms ("You don't have a tablet and all your symptoms just go away.") and elaborates that it takes "a good three or four months for that to happen", after which, the medication may still be evaluated as inefficacious ("and then sometimes your medication doesn't work"). She reports that it took three years, following diagnosis, before she was prescribed a suitable medication. Whilst no direct links were made with adherence, Diana's account highlights the complexity of medication adherence. Particularly, the sometimes lengthy process of finding a suitable medication appears fraught 
with potential obstacles to adherence, given the variable consumer responses to different medications and dosages.

In the subsequent extracts, interviewees attribute their maintained adherence to the effectiveness of medication in treating their symptoms, despite concurrently experiencing side effects. Thus, the effectiveness of medication exerted a greater influence on adherence than side effects for interviewees below:

Ruth, 31/07/2008

R: Yeah, you put on weight, because I've put on weight since I've been on medication. It's called Vansza. So that, coz my doctor told me it puts weight on.

L: Right, and so you've found then that that has happened to you?

R: Yeah.

L: And so that would be a negative effect?

R: Yeah, but what can I do? I'd rather have weight than be sick.

Ryan, 26/09/2008

$R$ : Yes. I'm sure-, I think all medications to some degree are a bit sedating or a bit uh, but um, but it's better to be on them than nothing, because to be on nothing is like um, you just can't function at all, you can't, you haven't got the uh, the concentration because your mind is so um, like uh, paranoid and not able to function, so any medication is better than none.

The above extracts downplay the importance of side effects. Ruth concedes that weight gain is a negative side effect and Ryan talks about the sedating side effects of medication. Ryan minimises the impact of sedating 
side effects by normalizing them; "I think all medications to some degree are a bit sedating". Ruth frames enduring the side effect of weight gain as the only realistic option for her (“what can I do?”). She then directly expresses a preference for experiencing weight gain over becoming "sick", which is implied will result from non-adherence and the associated lack of treatment for her symptoms. Ryan also constructs enduring sedation as the preferable option in comparison to non-adherence and a lack of treatment for symptoms ("but it's better to be on them than nothing"), which he associates with paranoia, concentration difficulties and compromised functioning. To summarise, these extracts reflect the viewpoint that the benefits of medication in terms of effectiveness in treating symptoms outweigh the risks in terms of side effects and adherence is therefore reinforced. Similarly, in the extract below, Cassie indicates that despite knowledge of the serious side effects associated with antipsychotic medication, the benefits of adherence in terms of enhancing her life outweigh these:

Cassie, 04/02/2009

L: How do you feel I guess generally, overall about taking antipsychotic medication?

C: I don't like it, coz I read an article in the paper years ago that it takes about 5 years off your life. But then I looked at it the other way, if I didn't take it, I wouldn't have a life so I take it.

Specifically, Cassie highlights that antipsychotic medication "takes about 5 years off your life". Whilst she evaluates taking medication negatively ("I don't like it"), she acknowledges that if she "didn't take it", she "wouldn't have a life". Whilst it is unclear whether she is implying that she 
was suicidal when symptomatic, or whether her functioning was so poor that she could not participate in life, she emphasises the significantly negative, potentially fatal, impact of non-adherence on her life and associates this with her adherence ("so I take it").

\subsection{Chapter summary}

The medication-related factors category is comprised of codes relating to the route of administration, storage of medication, side effects, efficacy and consideration of side effects and efficacy collectively. The side effects code and codes related to the effectiveness of medication in treating symptoms incorporated foci on the bodily experiences associated with taking medication. The impact that various medication-related factors exerted on consumers' functioning and their everyday lives was also apparent throughout the analysis.

Whilst the route of medication was not commonly discussed by interviewees, some indicated that a long-acting depot route enabled them to overcome the inconveniences associated with having to take medication on a daily basis. A depot route was also constructed as helping consumers overcome unintentional non-adherence, through forgetfulness. Regarding storage, some interviewees indicated that medication packs and dosette boxes enabled them to keep track of their adherence and at times, enabled them (or others) to intervene by addressing missed dosages.

A wide range of side effects were reported and, whilst they were often implicated in non-adherence, interviewees' tolerability of side effects varied, often depending on how much side effects hindered their lives. Consistent with past research, some interviewees stated that they experienced 
particularly intolerable side effects following being administered high dosages of medication, or when taking multiple medications, which often occurred during hospitalisation and frequently resulted in discontinuation. The effectiveness and ineffectiveness of medication in treating symptoms were associated with adherence and non-adherence respectively. However, in line with previous findings, interviewees frequently talked about side effects and efficacy collectively. That is, interviewees tended to weigh up the benefits of medication (treating symptoms and the associated impact on life) and the costs associated with medication (side effects and associated impact on life) in the context of expressing their past or present stances on adherence. 


\section{Chapter 7: Service-related factors}

The service-related factors category encompasses codes that relate to consumers' relationships with prescribers (psychiatrists and general practitioners) and interactions with other providers, such as case managers (social workers) and peer workers and services, such as community centres. Service-related factors were frequently raised in interviewees' talk about their experiences with antipsychotic medication. Interviewees often talked about both past and present experiences with service providers and services in relation to their adherence at different stages and how services could be utilized or altered to assist consumers to achieve better outcomes.

The most common service-related factor raised by interviewees in this study was the relationship between consumers and prescribers, referred to as the therapeutic alliance, which has been well established in the literature as an influence on adherence (i.e., Barnes et al., 2000; Dassa et al., 2010; Day et al., 2005; Frank \& Gunderson, 1990; Irwin et al., 1971; Lacro et al., 2002; Olfson et al., 2000;). The proceeding analysis helps to contextualize previous research findings, as interviewees elaborate how, and which, important elements of relationships with prescribers (and occasionally other service providers) influence their adherence choices.

Service providers, such as case managers and peer workers, and other services, including community centres, were also raised in interviewees' talk in relation to medication adherence. These services are not commonly individually associated with adherence in the literature, however, they may have been discussed in relation to interventions and featured in some recovery research (i.e., Liberman \& Kopelowicz, 2005). Perceptions of case 
managers ranged from helpful and supportive to unhelpful. Interviewees consistently spoke positively about peer worker services and community centres. Peer worker and community centre services were typically represented as supporting adherence and consumers' general well-being by interviewees and, moreover, greater peer worker involvement in the treatment model was encouraged.

The service-related factors analysis commences with the therapeutic alliance code, which concludes with a sub-code in relation to non-adherence as an expression of resistance. Following on from this, a code in relation to peer workers and community centres is presented. Extracts within this code were positively framed. Furthermore, interviewees frequently supported an increased role for peer workers in interventions to address poor adherence amongst consumers. The case manager code is presented after this.

\subsection{Therapeutic alliance}

The therapeutic alliance in this context refers to the relationship between the prescriber and consumer. Most of the interviewees in the present study were prescribed medication by psychiatrists and the rest were prescribed medication by general practitioners.

Some previous attempts have been made to identify the essential elements of a positive therapeutic alliance. In their analysis of service users' views of psychiatric treatments, Rogers and Pilgrim (1993) identified belief in treatment, the maintenance of hope, willingness to share information, avoidance of confrontation and punishment, mutual involvement in decisionmaking and accessibility to the consumer on the consumer's terms as essential components of a positive therapeutic alliance. Regarding 
adherence, studies have highlighted the importance of collaboration (i.e., Corrigan et al., 1990) and the duration of treatment from the same healthcare provider (i.e., Compton, 2007; Frank \& Gunderson, 1990; Geller, 2000; Liberman \& Kopelowicz, 2005). It has also been recommended that healthcare providers involve family members in treatment decisions to enhance adherence (Blahski et al., 2004). Moreover, studies have indicated that there may be a negative effect on adherence if service providers fail to empathise with consumers' reasons for non-adherence, or regard the consumer's illness as beyond repair (Weiden et al., 1991). Additionally, Moritz et al. (2009) found that $29 \%$ of participants in their survey study reported that distrust of their prescriber influenced their non-adherence.

This code is organised into sub-codes that reflect the elements of the therapeutic alliance that were considered important by interviewees in the present research. Specifically, consistent with previous findings, interviewees indicated that the power relations that operate within the therapeutic alliance influenced their adherence. They tended to contrast a collaborative alliance, whereby treatment decisions were shared, with an authoritative alliance, whereby interviewees perceived their prescribers to have control over their treatment regimens. Additionally, interviewees indicated that prescribers' interest in their experiences (as reflected through in-depth questioning) and their knowledge of relevant background information were important to them. Most importantly, interviewees overwhelmingly reported that the degree to which their prescribers tailored their medication regimens to their individual circumstances, including symptom fluctuations, stressful situations and lifestyle factors, influenced their adherence. Extracts that relate to these aspects of the therapeutic alliance are presented below. 
7.1.1 Power relations in the therapeutic alliance: collaboration vs. authoritarianism

Research has shown that most consumers want to participate in the process of medical care, highlighting the importance of collaboration in devising treatment plans (Mitchell, 2007). Whilst collaboration is considered a cornerstone of a positive therapeutic alliance, the degree to which collaboration actually takes place in clinical practice has been challenged by some research. In their survey study, for example, Gray et al. (2005) found that whilst consumers with schizophrenia were satisfied with their communication with mental health clinicians, they did not feel involved in treatment decisions and, rather, attributed adherence expression to being instructed to by their prescribers. Similarly, in their qualitative interview studies involving people with psychiatric illnesses including schizophrenia, Sharifet al. (2003) found that consumers and their families considered that they were not generally involved in managing their psychiatric illnesses and Rogers et al. (1998) reported that consumers felt that health professionals controlled their treatment.

Interviewees in the present study consistently highlighted the importance of collaboration. Their accounts also frequently indicated that a collaborative therapeutic alliance does not reflect their actual experiences. In the following extract, Travis highlights the importance of the therapeutic alliance to consumers' future outcomes and describes what a "good" relationship with the treating prescriber looks like:

Travis, 19/02/2009

T: You've gotta have a good relationship with your psychiatrist. Otherwise, you're not gonna get anywhere. Well you can, but it's not very pleasant. 
When you go there, you wanna be able to feel heard and understand what they're doing and what needs to be done.

L: Yeah and you said you've been able to negotiate your medication with her as well.

T: Yeah, you can, yeah. I mean, there's been times when they've said, look, we want it down a bit more and I've said, no, I'm not ready, and they've said, fine.

L: So do you feel like you have some sort of control over your treatment then?

T: Yeah of course, that's what they need to feel. They need to be a part of it, you know. It's themselves that are going through it. They need to have some say, you know. It has to be reasonable but they have to, you know, at least have explained to them why, you know.

Travis constructs a "good relationship" with the treating psychiatrist as encompassing listening ("you wanna be able to feel heard") and understanding of the rationale for prescribing medication ( "have explained to them, why, you know") as well as the treatment direction ("what needs to be done"). Prompted by the questioning, Travis also provides an example which illustrates how he has been able to negotiate his medication schedule in the past and assert control over his treatment by declining his psychiatrist's proposal to lower his dosage. Travis indicates that it is essential that consumers have some control over their treatment ("they need to be a part of it...need to have some say") considering that they will be consuming the medication ("It's themselves that are going through it"). Whilst he does not directly link these elements of the therapeutic alliance to his adherence, he could be seen to be referring partly to the consequences of non-adherence 
when highlighting the negative outcomes associated with a poor treatment alliance ("otherwise, you're not gonna get anywhere").

In the following extract, Amy also indicates that some of the components identified by Travis represent important aspects of the therapeutic alliance, associated with adherence outcomes. Amy provides more in depth detail about what an "authoritative" therapeutic alliance might look like as well, based on personal experiences:

Amy, 10/02/2009

A: I think negotiation uh, where the client or patient feels like they've not only had a say but, not taking control but has an equal say of at least their opinion is being equally considered. Um yeah, collaboration, yeah, that's a good word. Uh, what else? Yeah, uh it's not, (inaudible) or parent-like, it should be negotiated like you're a colleague or a friend that you met at work or something but not an unprofessional friend...That kind of, familiarity and once you've developed a good working rapport with your psychiatrist, I think it's important for your psychiatrist to build a good rapport or your occupational therapist or any treating health professional, develop a good rapport with their, uh, patient. The only thing you have to be wary of is developing too good of a rapport that um the patient can sometimes get transference from if you have (inaudible).

L: Uh huh. Um, ok so, I think that was really good. Basically having a collaborative relationship where you can discuss things, it's an open forum, as opposed to being I guess quite punitive and taking a more umA: Yeah see a lot of doctors act just like parents, parent, naughty child attitude or starts um, regarding compliance with their patients, I think they'd be far more successful if, I mean I've-, sometimes you do need a firm 
hand but not a heavy hand, like it is often practised today if patients aren't compliant, I can only speak for myself in that uh, I'm more likely to be compliant if the-, I mean sometimes you do need to get tough on me, I admit, and uh, my brother and sister, in particular my brother being the disciplinarian has driven me to tears at points because I've been ridiculous and I've needed a big kick up the bum and he does that for me but he's equally loving and caring as well.

\section{L: Yes.}

A: Um, but it's all balanced. Um, whereas psychiatrists? aren't always balanced when they're being authoritative with taking medication. Rather than being authoritative I think the patient needs to feel like they've had an equal say and that's being equally valued uh, as strongly as they feel about their opinion and like, it's been at least considered by the psychiatrist or treating medical health professional. They need to feel heard. Most of all you need to feel heard and that your opinion is being considered and maybe even counted in moderation with their medical health care.

Amy highlights several important components of a therapeutic alliance which she implies are associated with her adherence ("I'm more likely to be compliant if...)". Specifically, Amy emphasizes the importance of "collaboration" with her prescriber, comprising "negotiation" whereby she feels that she has some "control" or an "equal say" and that her "opinion is being equally considered". She additionally highlights the importance of consumers establishing a "good rapport" with their prescriber, whilst acknowledging the necessity of prescribers to be professional in their interactions with consumers. Amy contrasts a collaborative therapeutic alliance with one where there is a power imbalance, which she likens to a 
"parent, naughty kid" dynamic to illustrate. Amy constructs the prevalence of "authoritative", "punitive" prescribers as common ("a lot of doctors act just like parents") and particularly evident when consumers are nonadherent ("a heavy hand, like it is often practiced today if patients aren't compliant"). Amy could be seen to suggest that the power imbalance results from the consumer being non-adherent in the latter quote, whereas the alternative perspective is that consumers become non-adherent in response to an authoritative prescriber. She supports the need for prescribers to occasionally be "firm" with her to encourage adherence but indicates that such an approach is most effective when counterbalanced with "caring". Below, Anna also highlights the importance of the consumer being "listened to" and contrasts this with her experience during a hospitalisation of being administered an exceptionally high dosage of medication which impeded her ability to contribute to treatment decisions ("And not have them so bombed so that they can make um decisions"):

Anna, 18/02/2009

A: Hmm, I think too that the, the person needs to be listened to.

L: Aha.

A: And not have them so bombed so that they can make um decisions. Like, for my experience, I can't remember anything, you know, and the ECT treatment and how they got that, I don't know, but I obviously complied. But, normally I would never have consented to that.

L: Yeah but it's just like you were so out of it.

A: Yeah, I probably would've signed anything.

L: Yeah. That's a really important one, I think.

A: Um, but yeah. That- 
L: It's like giving the client some control over their own treatment, or giving them-

A: Could yeah, like give them like a small, a small dosage and maybe nice medications just to get them-, build them up slowly to a level rather than bombarding them with medication and you're just left to it.

Anna represents her experience of being over-medicated as compromising the capacity for collaboration in the therapeutic alliance. She reports an inability to recall consenting to another treatment, electroconvulsive therapy ("I can't remember anything"), which she received in addition to medication in hospital, due to the effects of the medication. Anna reflects regret in relation to receiving the treatment, stating, "normally I would never have consented to that". She suggests that rather than "bombarding" consumers with medication, initial treatment should involve a gradual increase in medication in order to ascertain optimum dosage. The extract raises concerns around the ethics of seeking the consent from inpatients who have been administered high dosages of medication, which in Anna's case, reportedly compromised her decision-making and, thereby, undermined the capacity for a truly collaborative therapeutic alliance. Similarly, in the next extract, Amy talks about her limited control over her treatment as her psychiatrist experimented with different medications and dosages:

Amy, 10/2/09

A: Not as a guinea pig, because too often people feel like guinea pigs with the medication, the psychiatrist's, is all keen to, "oh I'll give you new ones, oh 
there's this new one". They pump 'em up. You think, oh great, thanks, I'm a guinea pig.

Amy likens her experiences of being encouraged to trial "new" medications and dosages ("They pump 'em up") to that of a "guinea pig". By comparing consumers to guinea pigs, Amy works up a construction of consumers as psychiatrists' subjects and thereby emphasises the significant power imbalance between prescribers and consumers. Amy suggests that her experiences of prescriber-directed experimentation are common amongst consumers "too often people feel like guinea pigs". She could be seen to express frustration through the sarcastic remark "oh great, thanks" in relation to this practice. Whilst no direct linkages were made with adherence within the extract, it could be logically assumed that perceptions of oneself as being experimented on by psychiatrists may lead to non-adherence amongst some consumers. Indeed, Amy's experience of experimentation with multiple medications was common amongst interviewees. Whilst Amy constructed experimentation as imposed by the psychiatrist, however, others indicated that they trialled various medications in collaboration with prescribers, as part of the process of finding a suitable medication and dosage.

In the following extract, George talks about how his distrust of "doctors" due to his perception of them as having power over not only his treatment but his finances:

George, 14/8/o8

G: Coz like, I um, sorta lied, I said I wasn't taking more marijuana, you know, thought I'd just gave it up because I was feeling sick and every time 
she'd ask, I'd just tell her, no, no I don't take it. I don't like telling doctors things too much, you know? Coz if you tell 'em that you're taking it they'll just take your money off you, you know?

L: Really?

G: Yeah. I've, that's how I got the Public Trustee. Since I got outta there I've saved up \$18 ooo.

L: Geez.

G: (laughing) Imagine how much money I lost while I was in there. Yeah, cost them money to look after it. It's a big rip off; they've been on television and all, those bastards.

George states that he is dishonest with his prescriber ("I um, sorta lied") and strategically hides information relevant to his treatment due to fear of the consequences of not following his doctor's orders, namely: "they'll just take your money off you". George could be seen to position prescribers as punitive when consumers use drugs in addition to their prescriptions. He constructs his perception of prescribers as based on his past experiences of having to submit his money to the control of a public trustee and indicated that this occurs regularly to consumers by pointing out that it has been documented in the media ("they've been on television and all"). It could be argued that George's lack of openness with his prescriber compromises the prescriber's ability to assist George to address his drug use, which may represent a barrier to his adherence or, in the least, could contraindicate the therapeutic benefits of taking medication. Indeed, if discussions related to drug use are considered taboo, then so may be discussion related to nonadherence and other potential adherence barriers. 
In the following extract, Matthew also talks about his prescriber deploying punishment, but in relation to non-adherence. Unlike in previous extracts, however, he indicates that the threats his prescriber makes to punish him for non-adherence motivate him to remain adherent. Similarly, Gavin, in the latter extract, suggests that adherence could be enhanced if service providers warn consumers of the increased risk of hospitalization associated with non-adherence:

Matthew, 18/2/o9

M: Nah, that's my psychiatrist, yep. You don't take your clozapine, you're not very um, good and you're sick so straight to the lock up ward, he said, you know, if I don't take my clozapine. So I take it.

L: Straight to what?

M: Lock up ward at um-

L: Where's that? What's that?

M: There's a hospital lock up ward.

L: Gee.

M: I know.

L: So he's threatening.

M: I know, I know (laughing). Yeah, it freaked me out!

Gavin, 11/2/o9

L: How do you think consumers could be encouraged to take their medication?

G: Say to them, if you wanna stay out of hospital, you better take your medication. 
L: Ok now what's so bad about hospital? Everyone seems to say this, it's sort of like saying you're going to jail or something.

G: It is. Sometimes it is. Um, it's the people that are in there.

L: Yep. Like the workers?

G: Nah, the workers are alright. It's the patients. Some of them are very sick and um it can be very fearful, fearful all the time. You don't know if they're cracking up at you, or someone else or at the nurses.

L: Right. So it's scary. Hmm, um anything else then that's-

G: What, bad about hospital?

L: Yeah.

G: Um, just that you can't go nowhere, you've gotta stay there.

According to Matthew, his doctor appraises him negatively, as "not very um, good" and "sick" when he is non-adherent. He elaborates that if he is non-adherent, his psychiatrist threatens to send him "straight to the lock up ward" in hospital. Matthew reported that his psychiatrist's threats "freaked" him out and, thus, motivate his adherence ("So I take it"). Thus, whilst some consumers reported rebelling against punitive and controlling psychiatrists by becoming non-adherent, as will be illustrated in subsequent extracts, Matthew's account represents a different perspective, that fear as a result of service providers' threats of punishment for non-adherence can enhance some consumers' adherence. In the latter extract, when asked how to encourage adherence amongst consumers, Gavin proposes that service providers highlight the association between non-adherence and the risk of hospitalization to deter non-adherence ("Say to them, if you wanna stay out of hospital, you better take your medication"). Of note, the directive, "you better take your medication" could be seen as potentially threatening. Gavin 
concurs with the interviewer that hospital shares similarities with jail for consumers, adding that "you can't go nowhere, you've gotta stay there" and also states that inpatients can represent sources of fear due to their unpredictability ("You don't know if they're cracking up at you"). Matthew can be seen to suggest that the power imbalance operating within the therapeutic alliance between him and his psychiatrist, which manifests as threats of punishment for non-adherence, supports his adherence and, similarly, Gavin encourages service providers to warn consumers of the negative consequences of non-adherence to support adherence.

Interestingly, Gavin describes hospital as limiting consumers' agency and, thus, as a system in which significant power imbalances operate. This construction could be seen to partly account for his support for authoritative intervention, which may represent a means of avoiding more significant power imbalances that operate in hospital settings.

In the following extract, Brodie positions his prescriber as a knowledgeable expert, whom he entrusts control over his treatment:

Brodie, 21/8/08

L: So you know that time when you um, asked for your dose to be lowered, how come you asked to have it lowered?

B: I think coz I, I figured I could still be alright without 1omg but I guess not, but um, it was too low. It wasn't-, I don't think I was better at the time, um (inaudible) probably too soon, but like I said, it's not up to me, it's up to the mental health, up to the psychiatrist to prescribe all that stuff. So yeah I just do as I'm told and take whatever. 
Similar to the previous extracts, it is clear that Brodie's psychiatrist has control over his treatment regimen, which he seems to have accepted (" $I$ just do as I'm told and take whatever"). Brodie recalled previously requesting to have his dosage lowered as he "figured [he] could still be alright" which was then disproven by his experience ("but I guess not"). He could be seen to relate this experience to his present trust in his prescriber to manage his treatment. Whilst Brodie may appear to position himself as subservient to his prescriber, his decision to allow his prescriber to dictate his medication regiment could also be seen to reflect a sensible, rational choice and an attempt to ensure that his treatment decisions are not influenced by his mental instability. That is, in the context of mental instability, allowing the prescriber to have control over the treatment regimen may be more beneficial for consumers, thus, challenging whether true collaboration is a positive goal, when consumers' symptoms are florid and their judgment is potentially impeded.

\subsubsection{Interest in the consumer's experiences and background knowledge}

In the following analysis, interviewees raise the importance of prescribers asking them questions about their general experiences to acquire knowledge about their unique situations, in order to assist with adherence. This is contrasted with interviewees' experiences of prescribers focusing solely on illness symptoms or prescription information, asking the same questions week after week and, generally, adopting a more impersonal approach to treatment. In line with research which indicates that longer duration of treatment with the same prescriber influences adherence (i.e., Compton, 2007; Frank \& Gunderson, 1990), the last extract presented highlights how a rotational system, whereby psychiatrists work in a 
medication clinic for a brief period of time and then leave, compromises the capacity for prescribers to gain intimate knowledge of the consumer, which could be assumed to compromise their ability to then tailor the treatment regimen to the consumer.

Below, interviewees highlight the types of questions they think prescribers should ask them and contrast this to a lack of interest in consumer experiences:

Gary and Ruth, 31/07/2008

L: Cool, thanks. Thanks for that. Ummm, so are there any other ways that you think um health workers could help people, could assist people in ta-, to take their medications?

G: Well, umm, see I think that health workers don't ask enough questions, you know what I mean? Are you still taking your medication?

R: Yep.

G: They just ask you how you, you know, they ask you how are your symptoms and you tell 'em your symptoms and...you know like the psychiatrists I've had, they seem to be a bit ignorant, you know what I mean?

L: Mmm hmm.

G: They haven't explained themselves enough. They never seem to have the time to, to ex-, you know, how are you going? How are you going on this medication? This, that and the other.

L: Oh ok, so they'll just ask about how your symptoms are and not so much about your experiences with, of taking the medication.

G: Yeah, yeah, whereas Doctor Thas been pretty thorough with that, you know and the health workers I've had recently, they've been pretty good but 
like, years ago, when I went off my medication the psychiatrist, I don't know, he just, just wasn't a very good one, you know what I mean?

Oliver, 21/08/2008

O: And the psychiatrist just says the same thing: How does your medications? How is your appetite and all that? Have you smoking any pot, have you took any alcohol, you know?

L: Every time, you get the same questions.

O: Yeah, and they're like, every time we see them they ask you what medications you're on, it's like, check the notes. You've got it there.

L: Yeah. You'd think they'd know if they're, and especially if they're prescribing it.

O: Yeah you'd think they'd know and they wouldn't have to ask you all the time.

L: So you were saying that you find like, they just ask you the same sort of things.

O: Yeah they do.

L: What do you think would be useful for them to ask, or like, what sorts of things, how do you think it should be when you go and see your psychiatrist?

O: Well they should ask you, have you got any problems, have you got any concerns, have you got any worried about anything, you know. It's like, how's the last couple of weeks been? Is there anything been upsetting you and stuff like that.

L: Yeah.

O: Yeah so you know, to joke around, give a bit of advice, I dunno. 
L: Yeah, absolutely, because I guess if you go in there and you feel like, it's kind of impersonal maybe, like they're not really treating you as a person, you're just another number.

O: Some of them, I don't even feel like they care, they're just like, "yeah yeah". That's what it feels like. I had this bloke and it's like, it just sounded like he didn't care man. Yeah, I was in there 10 minutes and he just sent me out.

In the context of being asked about how health workers could assist consumers with adherence, Gary suggests that prescribers should ask consumers more questions, as they "don't ask enough", which is also illustrated through his elaboration that prescribers "just ask you how you, you know, they ask you how are your symptoms". In line with this, Oliver states that his prescriber "just says the same thing". He indicates that prescribers' questions focus on medication and dosage information and implies that prescribers fail to read notes prior to appointments. Gary could be seen to suggest that a past prescriber failed to assist him during a period of non-adherence by not asking enough questions and thereby assesses him negatively ("he wasn't a very good one"). Oliver negatively appraises prescribers who fail to provide a personal ("they're just like, yeah yeah"), considerate ("he didn't care") and thorough ("I was in there 10 minutes and she just sent me out") service. Gary and Oliver provide examples of the types of questions that prescribers could ask consumers to assist with adherence and their general well-being, such directly asking about their adherence ("Are you still taking your medication?"), experiences of taking medication ("How are you going on this medication?") and about any potential stressors ("have you got any problems/concerns/worries about anything?"). Oliver also 
indicates that friendly rapport would be appreciated (“joke around, give a bit of advice").

It was surprising that some consumers indicated that their prescribers did not ask questions about adherence or potential stressors which could lead to relapse, given the established importance of relapse prevention amongst people with schizophrenia. This may reflect time constraints and a lack of resources in the mental health system, which prevents prescribers from being able to spend time gaining information about consumers they are treating. It could be argued that there may be a role for psychologists in providing a more personalized service for consumers, whereby they can discuss stressors and barriers to adherence for example. Only two participants reported that they saw a psychologist.

In the following extract, Oliver highlights the difficulties of establishing a therapeutic alliance in the context of the rotating system of psychiatrists at a medication clinic:

Oliver, 21/08/2008

L: Ok so do you think that your relationship with your psychiatrist is important then?

O: Yeah, it is important, but it's like, every six months you swap and you get somebody new and it's like, when you start to feel comfortable and talking to 'em, they change it.

\section{L: Hmm.}

O: So they gotta do it longer.

L: Yeah, more consistency would be better.

O: See I have trouble talking to them. It's like the first couple of times I saw Dr M, I wouldn't speak to him. I was like, “yeah, yeah, yeah, everything's 
fine, everything's fine", and I was like, I was, half the time I was miserable as fuck. Sorry, excuse my French.

L: No that's alright. Um, ok so until you've got that relationship you're not gonna be as open with them, is that what you mean?

O: Yeah.

Oliver acknowledges the importance of a positive therapeutic alliance but constructs seeing a new psychiatrist "every six months" as a barrier to this. He elaborates that as soon as he starts to feel "comfortable" enough to talk openly with his prescriber, "they change it". Oliver explains that he experiences difficulties confiding in prescribers he does not know well and recalls that in the past, he failed to notify his prescriber that he was experiencing depressive symptoms ("I was like, "yeah, yeah, yeah, everything's fine, everything's fine", and I was like, I was, half the time I was miserable as fuck."). Oliver does not directly link a prescriber's lack of knowledge of his background and unique circumstances to non-adherence. However, it could be argued that consumers may be more likely to become non-adherent if they endure symptoms or side effects as a result of not talking about their experiences with prescribers, as this limits the capacity of the prescriber to tailor the medication regimen to address consumer concerns. Oliver recommends that consumers see the same prescriber for a more extended period of time ("they gotta do it longer") in order to improve communication in the therapeutic alliance.

\subsubsection{Tailoring the medication regimen to the consumer}

The previous sub-code highlighted the importance of prescribers having knowledge about their client, which represents an essential pre- 
requisite for tailoring medication for a client. Arguably an aspect of collaboration, many interviewees highlighted the importance of prescribers tailoring their medication regimens to their unique situations in order to reinforce adherence. According to Sperry (1995), tailoring the treatment regimen refers to individualising or customising information and scheduling to the consumer's personality style and circumstances and has been linked to adherence in research. Consistently, in the following extracts, interviewees talk positively about prescribers who tailor their regimens according to fluctuations in symptoms, the presence of situational stressors, side effects and their daily routines. Conversely, consumers often associated nonadherence with prescribers' failure to consider their unique circumstances or concerns in developing or revising treatment regimens.

Below, after having recalled a period of time when she experienced situational stress, Diana positively evaluates her prescriber's response to this:

Diana, 11/02/2009

D: So I put myself in a bit of a bad position and he came onto me and there was no one around you see and I didn't know what to do but anyways, I got out of the situation. It upset me. But like I said, my doctor helped me; he put me on some stronger medication.

L: Oh because he knew that that could be a trigger.

D: Coz I knew it was a trigger because everything that upsets me, I go, I get really crazy. It doesn't affect me straight away; it will affect me later on.

L: Yeah like stress, yep.

D: Because I wasn't sleeping also. I wasn't sleeping and I was, wasn't eating properly and that was affecting me really bad. You know, you go through-, you go into your moods again. 
L: Yeah. And so your doctor then helped you through that, increased the dosage and-

D: Yeah and then he took me off the medication as well. So he put me on it, and then he noticed I didn't need it anymore and he said go on a lower dose.

L: Which is good.

D: The other doctors wouldn't have done that.

Diana describes how her prescriber "helped" her through a difficult situation, which represented a potential "trigger" for relapse, by increasing her medication dosage. She elaborates that once the situation stabilised, her prescriber then lowered the dosage of her medication, thus, tailoring it to her improved mental state. Diana concludes that "other doctors wouldn't have done that", suggesting that previous prescribers have not been as flexible with the medication schedule, consistent with her account of past experiences with prescribers during her interview. Whilst Diana does not directly link her prescriber's tailored approach to treatment to her adherence above, her association between increasing medication dosage and preventing relapse reflected acknowledgment of the benefits of medication in this respect and, therefore, it could be argued that her prescriber's tailored approach to treatment reinforced adherence. Next, Gary talks about how his prescriber supported him to change medications due to experiencing side effects:

Gary, 31/07/2008

L: So was that the main, did you ever stop taking it because of some of these effects or would you just change? 
G: No, I spoke to my psychiatrist about it. We had a chat about the side effects I was suffering and he changed me the medication.

L: Oh, so your psychiatrist has played a role then in regulating...

G: Yeah.

When directly asked whether he became non-adherent as a result of experiencing side effects, Gary denies this and recalls that rather, he "had a chat about the side effects", following which, his psychiatrist "changed ... the medication". Gary's account of his relationship with his psychiatrist is suggestive of open communication, informing a tailored approach. It could be argued that by adopting a tailored, individualised approach to treatment by experimenting with different medications or dosages in instances when consumers are experiencing side effects, for example, prescribers afford consumers with more opportunities to be adherent. That is, if the only options for a consumer are to either remain adherent and persevere with side effects or to become non-adherent, they may be more likely to choose the latter; whereas, if given the option of trying a new medication for example, this could represent an attractive alternative.

There appear to be undertones of frustration and distrust in mental health professionals in the following extracts, in which Diana and Steve talk about their prescribers' failure to tailor medication to their circumstances:

Diana, 11/02/2009

D: They might put my medication up or might have brought it down but didn't apply to the way I was at the time. 
L: Yeah so it's almost like-

D: They're only covering their backs, they're responsible for me but they weren't gonna be there for me.

L: Like on a more personal level, do you mean?

D: No, no, just, just to get along alright, but um, a couple of things weren't going right but that didn't matter. She's taking,just as long as she takes her medication.

Steve, 04/02/2009

L: Um, what's been the problem with previous um, doctors and that?

S: I don't know, I just didn't like their attitude.

L: Yep.

S: Basically you go and tell them what's happening and they just didn't really care. You know? It's just like, see ya later, like they won't give you anything for you, it's just like see you later.

L: So you're just treated like another number basically.

S: Yeah, another, like \$10o you know, sign this so they can get paid, you know?

L: Yeah but you're not getting that personal kind of care or service.

$S:$ Yeah or service that you need.

Diana indicates that changes in her medication regimen in the past did not reflect fluctuations in her personal circumstances ("They might put my medication up or might have brought it down but didn't apply to the way I was at the time."). She elaborates that prescribers did not take into account difficulties she was experiencing, which were possibly medicationrelated ("a couple of things weren't going right but that didn't matter") and 
implies that they failed to take any action because she remained adherent ("just as long as she takes her medication"). Steve indicates that despite informing his prescriber of his circumstances, these were dismissed and his regimen was not tailored to his needs ("you go and tell them what's happening and they just didn't really care...like they won't give you anything for you"). Steve and Diana position prescribers whose practice does not reflect consideration of their needs as lacking genuine concern for consumers' well-being ("They're only covering their backs", "another, like \$10o you know, sign this so they can get paid"). Although neither Steve nor Diana associated their prescribers' failure to tailor medication to nonadherence in the above extracts, they both reported at other points in their interviews that they became non-adherent due to experiencing side effects which were not addressed by prescribers.

In the following extracts, Cassie and Oliver negatively evaluate their past experiences of prescribers failing to tailor their medication regimen to their schedules:

Cassie, 04/02/2009

L: So they didn't tell you. Do you find I mean I know you think your relationship with Dr A's pretty good now, and you've mentioned in the past that you've had some not so great relationships with health workers, psychiatrists and that. Um, what, what was that like early on? And what effect did that have on you?

C: Um (pause), some of them are out of touch with reality. Um, some of them aren't um, but I remember like, um, why can't you have time off to come and get your injection, like 2 hours off to get the injection? Why do you have to have to do it in out of work time? We can give you the sick 
certificate. And it's every four weeks or something. And uh, why can't you have um time off to come and see um, see the psychiatrist when you're working? Why did you have to do it in non-working time and have it after four o'clock? And um, people don't hang around for jobs that long. If they knew, they wouldn't employ me.

Oliver, 21/08/2008

O: Yeah, and I'll, I was I was running uh, a couple of days late and it's like, come and take your injections you know, just cool like, you need your injections, come and take it now sorta thing and it's like, (noise), I'll do it on Thursday, you know, today's Tuesday, I've got an RDO Thursday so I'll be in on Thursday and it's like, "No, that's not good enough. You come in now" sort of thing, like he was bossy sorta thing.

L: Like a teacher it sounds like

O: Yeah.

L: So he didn't seem concerned about-

O: No, I tried to explain to him I'm working, I'm fine at the moment and it's like, two days won't hurt.

Cassie directly positions some of her past prescribers as "out of touch with reality". Cassie and Oliver report that their prescriber and social worker, respectively, failed to take into account their work commitments when arranging medication appointments. They both indicate that past prescribers have held unrealistic expectations that they should be able to prioritise medication appointments over employment commitments and that prescribers were inflexible in organizing more suitable times. Whilst they do not link prescribers' rigidity and failure to consider other commitments 
directly to non-adherence, it could be argued that if appointments consistently interfere with other life commitments, some consumers may be more likely to become non-adherent. Thus, it may be important for prescribers to tailor the medication schedule around consumers' other commitments, such as employment, in addition to tailoring it to symptom fluctuations and situational stressors.

Whereas previous extracts have emphasized the importance of tailoring medication regimens to consumers' situations, the following extracts highlight the importance of prescribers tailoring treatment information to their level of understanding or intelligence:

Brodie, 21/08/2008

L: How do you think health workers could assist in I guess, well encouraging or helping people to take their medication? What sort of role do you think health workers could have?

B: I think not to patronise some people, coz I've seen it happen before.

L: Patronising people?

B: Sometimes yeah. I'd like to think I've got some brains, but some people, they're not educated or anything, so it's probably harder for them. So yeah, but I think the health workers should individually, just um, get to know their patients a bit more than what they should I think. That's why me and Dr A get along well now, because, I just said to her, look I know I've got this thing that lots of other people have but I don't want to be talked down to like I'm stupid or anything.

Amy, 10/02/2009 
A: One of my mental health nurses, uh, she was looking after me and was really good at explaining, like from, because I've done biology in year 12 and I'm sort of, I used to do a mental health course at Tafe, non-clinical, um, he explained how the medication breaks do ? in your body, what it's made of and its purpose. And sort of like, the biological, medical properties of it...Um, because he knew I was intelligent enough, um, that really helped and encouraged me to take it more, because I had more thorough awareness.

When asked directly about how health workers could assist with adherence, above, Brodie responds "not to patronize people", as has been his experience in the past. He elaborates that prescribers should "individually, just um, get to know their patients a bit more" and thus target information accordingly. He implies that the therapeutic alliance with his prescriber improved following a conversation in which he told her to communicate with him as an equal ("I've got some brains...I don't want to be talked down to like I'm stupid or anything”). In line with this, Amy recalls how a mental health worker assisted with her adherence ("that really helped and encouraged me to take it more"), by acknowledging her intelligence and preexisting knowledge ("because he knew I was intelligent enough") and, thus, explaining the mechanism of medication to her in appropriate terms. Several interviewees talked about prescribers making assumptions about their intelligence or capacity to process information and, therefore, failed to provide sufficient information regarding their diagnosis and the rationale for the treatment prescribed. As can be seen in the following extract, several interviewees also indicated that prescribers often questioned the validity of 
their concerns or failed to take consumers seriously, possibly on the basis of such assumptions:

Diana, 11/02/2009

D: See if you slipped, they didn't care. You just get, that's what I got, slipped and slipped and slipped and then, until I went off my medication and then I just told 'em straight out, I'm low on medication, he didn't get cross at me or anything. He didn't hear, I know he was tired, because you can't force the patient to go on it, you can't put 'em in hospital but he could've been a bit more open.

L: And understanding and maybe even talk it through, like why you decided to go off it.

D: Yeah, yeah, there's so many things that he could've done but he left all the talking up to me and he didn't do anything to-

L: Yeah.

D: Every time I come off the medication, they wouldn't do anything about it. They wouldn't talk about changing. They wouldn't, you know, it's sort of really hard.

Despite making her non-adherence explicit in the past ("I went off my medication and I just told 'em straight out'), Diana describes how her prescriber failed to intervene ("he didn't get cross at me or anything"). She positions prescribers as largely indifferent to her adherence statuses by stating, "they didn't care". Diana could be viewed to suggest that her prescriber did not listen to her, through the statement, "He didn't hear", followed by acknowledgement that prescribers "can't force" adherence, but that her prescriber "could've been a bit more open" to discussing non- 
adherence. She constructs her experience of prescribers failing to act on knowledge of non-adherence as typical ("every time I come off the medication, they wouldn't do anything about it"). She also suggests that her prescriber had the resources to respond to her non-adherence helpfully ("there's so many things that he could've done"), including by discussing "changing" medications. Below, Rachel and Diana talk about social worker and prescribers' inaction in relation to their mental health and adherence:

\section{Rachel, 25/02/2009}

$R$ : They [social workers] need to interact with their clients a bit more, you know. Try and find out how their clients are going on a regular basis, not leave it to the point where the clients need hospitalization before they get involved with their clients. That's what really annoys me with my worker.

\section{Diana, 11/02/2009}

D: It wasn't until I got really bad that they [mental health staff] actually decided to do something about it and when he decided to do something about it, it was that I was to go to the hospital and take the tablets, not try to get me to take my tablets. They never tried to get me to go on the medication earlier. They didn't say to me, oh look, I think you're struggling, you should try to get back taking your medication or we can try to change it.

According to Diana, her prescriber only intervened once she relapsed ("until I got really bad") and the intervention involved admitting her into hospital, where she was required to take medication under supervision, thus, adherence was imposed. Rachel implies that her social worker also failed to 
intervene until she relapsed and was hospitalized ("leave it to the point where the clients need hospitalization before they get involved with their clients"). Diana highlights the lack of acknowledgement of her nonadherence, lack of encouragement to take her medication or an attempt to address non-adherence by changing medications ("They didn't say to me, oh look, I think you're struggling, you should try to get back taking your medication or we can try to change it.), whereas Rachel reports a general lack of involvement and recommends that workers "Try and find out how their clients are going on a regular basis", in contrast to her experiences. In summary, both Rachel and Diana could be seen to would have liked to have received adherence assistance sooner than they did.

Indeed, failure of prescribers to intervene early to address adherence was a common experience amongst interviewees. It seems that although adherence to antipsychotic medication is a recognized problem amongst health professionals, often, little action is taken to address this issue, except in extreme cases such as relapse or suicide attempts via overdose. As with other participants, Matthew talks, below, about his experiences of other service providers (in addition to prescribers) failing to intervene, despite his attempts to seek help. Matthew positions service providers including prescribers as indifferent and also points out the potentially detrimental consequences of failure to intervene early:

Matthew, 18/2/09

M: It seems like they don't care here. That's how it's trained here. They don't really care, no. 
L: Mmm and what effect does that have on you? I imagine you'd feel almost like you're not being um, treated like a human being almost, wouldn't it?

M: When I first got sick, there was a lot. It was better when I first got sick. But as time's gone on, it's worse, yeah.

L: Yeah, so what's it like?

M: The service is just out the window now.

$L:$ What are they like then now?

M: Like I said, the hot chocolate and that.

L: So it's like you don't feel like they're really-

M: They don't help. They're not caring and they don't help.

L: That's pretty bad because for some people that could be detrimental, like if they're reaching out.

M: Well some people could be overdosing, like take a whole pack of pills and are overdosing and all that comes is an ambulance, if you ring an ambulance. They don't, or they can rush you to emergency.

Matthew states that mental health service providers "don't care" and "don't help" and generalises his perception by suggesting that a lack of caring is reinforced through training for service providers. His reference to "hot chocolate" is in the context of his experiences of contacting a crisis intervention telephone service for people with mental health problems in the past, when acutely ill, and of being told to either take a bath or drink a hot chocolate by the service provider. Matthew elaborates later on that consumers' circumstances could be serious ("people could be overdosing") in which case, he constructs emergency ambulance service as the only reliable form of intervention, provided that the consumer has contacted them ("all 
that comes is an ambulance, if you ring an ambulance"). Thus, similar to the previous extracts, Matthew indicates that it is not until consumers take extreme measures, such as "overdosing", that they are taken seriously and can access services but even then, the prescriber is positioned as absent from the intervention ("all that comes is an ambulance").

Matthew acknowledges that when he first became sick, he had access to many services and seemed satisfied with the mental health system ("When I first got sick, there was a lot") but noted that "as time's gone on", mental health services have progressively worsened to the point that it "is just out the window now". Thus, he could be seen to imply that there is either an absence of services for consumers or that consumers are unable to easily access services that are available, with the exception of when they are first diagnosed. Indeed, another interviewee (Ryan, 26/9/08) indicated that he "initially" had a case manager following his first episode, however was told that due to heavy case loads and because he "was deemed a difficult case", paradoxically, the service was retracted. Although Matthew does not make a direct association between poor experiences with service providers and nonadherence, his portrayal of service providers and the absence of services accessible to consumers after diagnosis could suggest that consumers may not receive assistance as required when encountering adherence problems. It may be the case that like Matthew, consumers who are non-adherent may actually want (and actively seek) help but are not receiving it, which has not previously been explored in the literature.

In one of the following extracts, Bill recalls that his prescriber failed to act to modify his medication regimen when he reported serious side effects. Amy also states that her psychiatrist typically encourages her to remain 
adherent to her medication, rather than changing medications, despite her complaints that it is ineffective. Amy reports that as a result, she often does not return to her prescriber and becomes non-adherent. During his interview Bill reported past non-adherence associated with side effects, which could have potentially been prevented had his prescriber intervened appropriately.

Bill, 13/2/o9

B: Uh, Stelazine, uh, (pause), yes, with the Stelazine, with the Stelazine, I uh I, I exhibited, they have 20 side effects. I exhibited all of them.

L: Ohgosh.

B: From the breathing to the aching and the, to everything, everything that was in it and that was, I showed that to the doctor and I said, I screamed it out at my GP, I said, what's going on here? And so they kept on persevering with it, they kept on persevering with this Stelazine and-

L: In spite of you getting all of those side effects?

B: All of those side effects. And the doctors, do you know what the psychiatrist's response was? Oh, it's just product information.

L: But you were experiencing them. That's not validating what you're experiencing.

B: That's what I was experiencing, exactly. I mean, I went to a meeting of AA once and a bloke was there and I said to him, old John, I said, I think the doctors are experimenting with me and he said, oh, and the bloke chips in and he said, look, we know all of the side effects, we know all of them. We just don't know how you will react to them. And what I thought was well, I react to them so you should respond...That's why, when you say you're not happy with something, I know they persevere with them for a while because 
it seems to make them well, but when you say you're not happy with them, they don't try you on something else because, I dunno why. Why?

L: I mean, it's not really a collaborative relationship then, it's so top-down. It's like, well you've gotta take this regardless of how it makes you feel. B: That's right. That's what's happening at the moment but that's alright.

Amy, 10/2/09

A: Um, if my medication's not working with me I can usually negotiate quite well with my psychiatrist, reasonably well. Say ok, it's not working, I want to try something else. But I've gotta push really hard with my-, I can't just say, ok, this isn't working, she'll say, stay on it, stay on it and I might not go back. If they keep saying, stay on it, stay on it, stay on it, I think it's bad for me because I'll go off it anyway.

Bill indicates that his GP and psychiatrist failed to acknowledge his concerns about experiencing significant side effects ("they have 20 side effects. I exhibited all of them... I showed that to the doctor") by dismissing the side effect profile as "just product information" and "persevering" with the prescription despite complaints. Whilst Amy states that she is able to "negotiate quite well" with her psychiatrist, she elaborates that she has to "push really hard" to have her regimen modified. Additionally, Amy could be seen to indicate that her psychiatrist occasionally dismisses or fails to take into account concerns and, rather, encourages her to "stay on" the medication. Bill could be seen to express frustration and confusion as to why doctors "persevered" with medications in the face of his dissatisfaction (“when you say you're not happy with them, they don't try you on something else because, I dunno why. Why?") and Amy indicates that telling 
her to "stay on" her medication is "bad" for her. Amy elaborates that such an approach leads to her non-attendance to appointments ("I might not go back") and non-adherence occasionally ("I'll go off it anyway"). Bill, on the other hand, does not directly associate his psychiatrist and GP's failure to tailor his medication to his circumstances (i.e., to change medications due to side effects) to non-adherence. Rather, he seems resigned to passively accept the terms of health workers (L: "It's like, well you've gotta take this regardless of how it makes you feel." B: “That's right. That's what's happening at the moment but that's alright."). Many interviewees reported similar accounts about not being listened to and, rather, being asked to persevere with medications in spite of experiencing distressing side effects or perceiving medication as ineffective in treating symptoms. It appears that tailoring medication regimens to the individual needs of consumers does not occur consistently, which may have significant implications in terms of adherence.

\subsubsection{Non-adherence as resistance}

Interviewees occasionally stated that they intentionally became nonadherent to their medications in order to gain prescribers' attention, express resistance to prescribers or to prompt them to change their practice. For some consumers, non-adherence represented a means of them asserting control over their treatment regimen, when there was a perceived power imbalance in the therapeutic alliance. This is consistent with some previous qualitative research related to adherence amongst people with schizophrenia. Sharif et al. (2003), for example, found that the desire for independence and self-control were major influences on participants' non-adherence. This manifested through rebellion and resistance to take medication in the context 
of a lack of involvement in illness management or a coercive treatment approach from the prescriber.

In line with Sharif et al.'s (2003) analysis, below, Diana and Anna construct stopping medication and attempting suicide by over-dosing on medication as means of capturing prescribers' attention and making them listen:

Diana, 11/02/2009

D: Mmm, well the only way you talk back to these doctors is if you go off your medication. Then they have to do something because they're responsible for you

L: Yep.

D: They've gotta do something for you or they, you know, they feel responsible and you, you know, because you're their, yeah. It's not, it's not good.

L: Yeah.

D: It's not good to have to get real bad to get treatment...And the only thing you can do to improve your medication and get your psychiatrist to listen is to go off it. And you know it was wrong, but what can you do?

L: To make a statement.

D: Well you have to. You think if I'm taking medication, you just take more, you suicide. If your doctor wasn't listening to you and you're on this bad medication the only way you'd stop yourself is by taking yourself off and if you're on the injection, well you've just gotta overdose because the only way you can get out of this is to kill yourself. I mean I know it sounds drastic but it's the only thing I know. 
Anna, 18/02/2009

A: Yeah and I think too that a lot of that, well in my experience, a lot of that has been because I've been um, pushed aside.

L: Oh yep.

A: Then you think, oh what'll I do? Oh, I'll take some more pills.

L: Aha.

A: Um, yeah, definitely, that's been a reason for me, um medicating too, like after talking to ACIS or um, uh, someone in the mental health field because I've just felt like I've been pushed aside.

L: You'd think like, even the people who are there to help, aren't helping. A: $M m m$.

L: So yeah, where do you turn?

A: Yeah. It's a vicious circle really.

Diana indicates that consumers do not receive adequate treatment until their condition significantly worsens ("It's not good to have to get real bad to get treatment"). She constructs non-adherence as the "only" means for a consumer to "talk back to these doctors" and "get your psychiatrist to listen". She adds that for consumers who take depot antipsychotic medication, "well you've just gotta overdose", then frames suicide as a more appealing option than persisting with a "bad medication" ("the only way you can get out of this is to kill yourself"). In the context of being asked about past overdoses, Anna attributes these to feeling as though her concerns were dismissed by health workers ("a lot of that has been because I've been um, pushed aside... Then you think, oh what'll I do? Oh, I'll take some more pills.”). 
In both extracts, a poor therapeutic alliance is, thus, constructed as a strong, perhaps dominant predictor of non-adherence amongst consumers who desire collaboration in their therapeutic alliance but experience an absence of it. According to Diana, prescribers are prompted into action when consumers become non-adherent as "they feel responsible". She acknowledges that overdosing and non-adherence represent "drastic" measures but reiterates that they are the only ways that she can effectively communicate dissatisfaction with her treatment regimen to her prescriber. Anna describes entering the mental health system following overdosing as a "vicious circle", suggesting that she will receive treatment in there, but may re-enter the system once she has returned home and potentially experiences mental health or medication problems which workers fail to address. In the following extract, Amy also talks about communicating resistance to "pushy" prescribers through non-adherence in order to assert control over her treatment regimen:

Amy, 10/2/09

L: So you've mentioned that um, your psychiatrists in the past have been a little bit pushy with your medication and you've found that that's been really off-putting for you.

A: Yeah because I'm extremely stubborn. To me, it's like they were like pushy sales people, in terms of like, oh yeah, kick backs from uh, drug companies and all that. Coz you know how doctors go to these workshops and you know, there's like secret little political kick-backs and stuff like that. I mean, they're very highly professional and everything as well, all the doctors, but sometimes they can be too pushy, worse than a pushy sales person. 


\section{L: I can understand that you might-}

A: If they had more of a gentler approach, firm but gentle, rather than threatening like with guardianship boards and stuff, which really scare you, and then you just go, totally the opposite way, to the opposite extreme...Yeah, well when they're arrogant that creates a (inaudible) that's a bit unhelpful, because then their kinds of practice reciprocates and they try one up and they, if a client feels-, particularly me, if I feel devalued, and my opinion, especially because I'm intelligent, I'm too smart for my own good sometimes, so if Ifeel de-valued I'm then going to try and prove my worth by being stubborn and that sometimes results in making the wrong choices.

L: OK, so sometimes you might have gone off your medication to kind ofA: To prove a point (laughing).

Above, Amy highlights the paradoxical effect of prescribers who strongly promote adherence and threaten punitive consequences for nonadherence ("threatening like with guardianship boards and stuff"). She likens prescribers who adopt such an approach to a "pushy sales person" and questions whether their practice is motivated by political incentives ("kick backs from uh, drug companies"). She highlights the practice as involving a power imbalance ("they try one up") in favour of the prescriber. Amy constructs such an approach as "unhelpful" and causing her to feel "devalued". Thus, she states that her response is typically to become nonadherent ("and then you just go totally, totally the opposite way, to the opposite extreme”). Non-adherence is represented as a means of gaining control over the treatment regimen ("their kinds of practice reciprocates") in a way that makes a statement to the prescriber ("prove a point") and to 
"prove my worth". Amy suggests that a "gentler approach, firm but gentle" would be more effective in encouraging adherence as opposed "threatening" and using scare tactics. It could be assumed that such an approach may not evoke as much resistance, in the form of non-adherence, amongst consumers motivated to prove their worth, like Amy.

\subsection{Community centres and peer workers}

Interviewees commonly reported that contact with other consumers or with peer workers could have a positive role in assisting with adherence, due to shared experiences of taking antipsychotic medication and mutual understanding of mental illness. Peer workers, in particular, were often described as a valuable resource for consumers, particularly because they were presumably stable and adherent, thus representing positive role models for consumers. Additionally, one interviewee who also worked as a peer worker framed this experience positively and could be seen to imply that it enhanced his sense of purpose and life in general. This is consistent with recovery research, which posits that participation in consumer-run programs, including peer worker programs, may engender a feeling of empowerment (Liberman \& Kopelopwicz, 2005). Liberman and Kopelowicz (2005) extend this argument further, stating that such feelings of empowerment may be instrumental in motivating a person to sustain treatment.

In the following extracts, Travis and Diana highlight the benefits of consumers socializing with each other at community centres and Robyn states that she has benefited from courses run at community centres aimed at assisting consumers to cope with their illnesses: 
Travis, 19/02/2009

T: Yeah, that's great and if you've got that, the tablet's working alright, you've got the family support, you've got a good social friends, that's the best chance you've got, you know. It's not always that simple though and people are surrounded around different situations, different things and um, and that's why this place [Club 84 community centre] is a good place because it gives them a chance to get away and be around people that understand.

L: Yeah definitely, people who have gone through similar things.

T: Yeah and if they don't wanna talk, that's cool, leave them alone, you know you haven't got all these people saying, snap out of it.

Diana, 11/02/2009

D: Going to Club 84's good because you get to talk to other people with mental illness and you get to do um, classes if you know like. There's one class, Headstrong, they have and it talks about mental illness. It doesn't matter how many times you go there, you learn more. I don't go quite as much as I used to but I actually think if they had um, you know, more groups, where you sit around in a group and you talk about your mental illness and how it has affected your life.

L: Your experiences?

D: Yeah, but they don't have that. Even if you had a psychologist do the course.

L: To run the group?

D: Yeah so you could talk about your different experiences and that other person might actually do something that you would like to do, so they can then tell them the steps of how they actually do it. 
Robyn, 18/2/o9

R: Club 84's a helpful thing to me. They have courses there during the day and that you can do. Learning to cope with your mental illness and stuff.

Travis, Diana and Robyn all portray a particular community centre positively. Specifically, Diana and Travis both describe it as "good" and Robyn describes is as a "helpful thing" to her. Travis highlights how community centres, whereby consumers can socialize can be especially beneficial for consumers who lack positive social supports. He suggests that consumers are more likely to "understand" fellow consumers and be patient with one another as opposed to expecting them to "snap out of it". Diana promotes group-based interventions, involving consumers sharing their illness experiences ("I actually think if they had um, you know, more groups, where you sit around in a group and you talk about your mental illness and how it has affected your life.") as a means of assisting consumers with adherence. She elaborates that sharing experiences may enable consumers to problem-solve various issues, as they can adopt strategies that other consumers found helpful ("that other person might actually do something that you would like to do, so they can then tell them the steps of how they actually do it.”). Robyn suggests that the group-based educational courses run at the centre have been beneficial to her. Thus, in addition to community groups providing a safe environment for consumers and an opportunity for socializing, through sharing experiences, consumers may learn new strategies for managing their illnesses and their medication.

In the following extract, in the context of being asked how to assist with medication adherence, Cassie also recommends community centres, 
specifically the peer worker service they provide, as beneficial particularly to "young" consumers. Both Cassie and Ryan below highlight the benefits of peer workers' abilities to relate to consumers' experiences:

Cassie, 4/2/09

C: Um, it's gotta be people that can relate to people, you know what I mean? That get through. It can't be just like some official talking and telling him that, because you just switch off and you don't wanna know. It's gotta be someone that you can relate to.

L: Definitely.

C: That's why peer workers can be a good thing. Peer workers talking to young people would help I think. Like if they had a visit to Club 84 [community centre] and met all the people and then went back and then talked to a peer worker or someone, I reckon that would help.

Ryan, 26/9/o8

$R$ : This [first episode, upon diagnosis] is where clinical staff and peer workers can come and say, or definitely peer workers can say, this is my experience and just try talking to the person and be honest with you and they can kind of like say, well it sounds like you've got something similar to what I've got, so uh, they'd be afraid to talk to you and their psychiatrist about it.

Cassie directly portrays peer workers positively (“a good thing”). She positions peer workers as more likely to positively influence young consumers than health professionals, as they are easier to "relate to". Similarly, Ryan highlights the role for peer workers amongst first episode 
consumers, who can share their "similar" experiences. He suggests that first episode consumers may be "afraid" to talk to other service providers including psychiatrists. Ryan could be seen to imply that by sharing their experiences, peer workers normalize mental illness for first episode consumers, who would likely be feeling overwhelmed upon diagnosis. Cassie additionally contrasts being relatable with "official talking", which she associates with consumers losing interest and failing to process advice ("you just switch off and you don't wanna know").

In the following extracts, Amy and Travis highlight how peer workers can provide positive examples to other consumers, which may assist with adherence:

Amy, 10/2/09

A: Um, it also helps if somebody's very respecting and (inaudible) and they've got sick and I mean, on the one hand, clients are, if they're admitted to (inaudible), if they are a peer worker or something, gets sick you know, you sort of lose hope that person's confidence and professionalism and ability and I think that came up because, that was, "Oh I recognize you from somewhere" and now you hear of someone that's really fighting, you say, oh well now you'll see me get better. So um, if they recognize that oh yeah, everyone gets sick, you know, like I've found it seems to help people when I say, they felt embarrassed by being sick or they're a bad person or, you know, I just say to them, a doctor needs a doctor and a priest needs a priest and a psychiatrist needs a psychiatrist. And you can see the weight lifts off their shoulders a bit. It seems to make them feel a bit better. It's sort of like an epiphany. 
Travis, 19/2/09

T: I think you need peer workers to show them that it works, that it can work and eventually it probably will work. It just takes time. They're the only people that can show that it works. Just say, look, you look unwell and you look unhappy. You can change it. Just stick with it. You can work with us. You can get better. Hopefully (laughing).

Amy talks about how peer workers can normalize relapse for consumers, which can help to reduce the stigma associated with symptom fluctuations ("if they are a peer worker or something, gets sick you know...they recognize that oh yeah, everyone gets sick"). Amy suggests that normalization can provide consumers with relief ("the weight lifts off their shoulders a bit. It seems to make them feel a bit better.”), perhaps as it addresses feelings of isolation and failure. Whilst she acknowledges that witnessing a peer worker relapse may compromise their "confidence", Amy could be seen to imply that by "fighting" back from relapse and expressing determination to stabilize ("now you'll see me get better"), peer workers can inspire other consumers. Indeed, at a later stage in her interview she described peer workers as "inspirational; especially if they've been a volunteer or whatever and gone onto further study". Travis represents peer workers as proof to other consumers that antipsychotic medication works ("They're the only people that can show that it works"), and as positive role models for consumers, who can genuinely promote adherence based on their experiences.

In the following extract, Ryan highlights how consumers' illness factors, such as paranoia, can compromise their relationships with service providers such as psychiatrists, which can thereby impede service providers' 
utility. He then suggests that there is a "bigger role" for peer workers who may be better equipped to communicate with consumers:

Ryan, 26/9/o8

$R$ : Um, yeah. Um largely, uh the problem is with people who aren't diagnosed, uh, it may be their first time, uh, because they haven't experienced it before they may think they're having some sort of spiritual experience like I did. Also, the problem being that uh, I was, because I was having a spiritual experience, I couldn't trust anyone, I thought everyone was out to get me, I wasn't open, uh I wasn't honest with others, whether it be psychiatrists, whoever it was, even other $1 G$ Ward mates for instance.

L: You thought you couldn't trust them.

R: Yeah I wouldn't trust them so I wouldn't tell them anything. So without that communication, they-, well you can't do much about it unless you pick up on the strange behaviour or the strange speech. I believe there's a bigger role for say um, peer workers to be in each, to take a bigger role or to be more positions in each, like in say, community centres, mental health hospital wards and government welfare agencies, just like, so there's like a peer worker; someone told me it'd be good if there was a peer worker at every stage of the journey.

L: Like because there's rapport.

$R$ : Yeah so there's a rapport there. At $1 G$ [mental health hospital ward] $I$ found they were good to talk to and they helped, so um, the, like get referred to a place like Club 84 [community centre] and the peer workers there, about two, the uh, the members.

L: I guess you could also be like a role model to people as well. 
R: Particularly for using strategies to control or help the illness. Yeah, it's a hard nut to crack but the idea of um when you talk about uh, when you're first unwell, uh about how to do your intervention, yeah that's, I wish I could help you out more there, but it's a hard one.

Ryan highlights how in the absence of open communication with consumers, as a result of potential denial of their illnesses which is associated with a lack of "trust" of health workers, service providers may lack sufficient information to provide effective treatment. He recalls that when he was hospitalized in the past and experienced paranoia and denial of his illness, he experienced peer workers to be "good to talk to" and "they helped". Ryan could be seen to suggest that consumers may have more open dialogue with peer workers, which could enhance their engagement with services and thus, potentially their adherence. With prompting, Ryan states that peer workers provided useful assistance with "strategies to control or help the illness". He promotes a greater role of peer workers in community centre settings and raises the possibility of peer worker involvement "at every stage of the journey", including government organizations that are not directly involved in mental health.

In the following extract, Rachel can be seen to suggest that health workers lack credibility due to their lack of personal experiences related to mental illness and antipsychotic medication:

Rachel, 25/2/09

L: Cool. Um, do you think health workers could have a role then in helping with adherence?

$R$ : I reckon they should. 
L: Yep. Do you think they're helping at the moment?

$R$ : Not really. I don't think they've got much idea about their patients with their medications. I think they read a lot of text books.

L: Yep so they know maybe about what's in the medications and how they're supposed to work but they're lacking maybe in people skills or something, is that what you mean?

R: Yeah. I'll give you an example. My daughter's sexual abuse counsellor is a young girl, she's fairly young. Never had children in her life. And she sits there and tried to tell me, I understand where you're coming from and it's like, no you don't. I'm not being rude, I don't wanna be rude, but how do you know what I'm going through when you haven't got children of your own? Oh, but I've read it in text books, but text books are nothing like hands on experience.

L: Yep so then-

R: Oh yeah, that's what really annoys me. Oh yeah, I understand. Oh yeah, I understand fully, I can, yep, yep. And it's like, how can you say that when you haven't experienced it yourself?

L: Hmm, exactly. You can't fully understand what it's like.

R: No and that really gets up my goat. It really gets up my goat. That really annoys me.

L: I mean I guess it's good that she's trying to put herself in your shoes.

$R$ : Oh yeah she's a lovely person. She is beautiful, a beautiful person and my daughter really likes her but I hate it when she says, I understand. Yes, I can relate to what you're saying and it's like, how can you say that when you've only read text books? 
Rachel describes health workers as somewhat unhelpful and indicates that they lack understanding of consumers ("I don't think they've got much idea about their patients with their medications."). She likens health workers promoting adherence to a young counsellor with no children offering parenting advice. She can be seen to discredit the value of theoretical knowledge ("I think they read a lot of text books", "you've only read text books") in comparison to "hands-on experience" and expresses frustration in relation to a workers' assertion that she "can understand" her situation ("how can you say that when you haven't experienced it yourself?...It really gets up my goat"). Whilst Rachel does not directly suggest in the extract that peer workers could provide a more valuable service, one could assume that she may be more receptive to such a service considering she values "hands-on experience".

In the next extract, Travis, a peer worker, states that he can "understand" the majority of what consumers say due to his shared experiences, but acknowledges, nonetheless, the importance of a "different point of view":

Travis, 19/2/o9

L: Do you think health workers can have a role in assisting with adherence? T: Oh yeah, definitely. Especially the peer workers because I can talk to someone with a mental illness and I can understand everything they say. Most of it, anyway. But um, it is good to have that person that's well looking at it from a different point of view, because you do need that person.

In the context of being asked whether health workers can assist with adherence, like many interviewees, Travis suggests that peer workers 
"especially", could fulfil this role. As the previous extract suggested, Travis constructs the therapeutic effect of peer worker intervention as directly linked to his ability to relate to or "understand" the consumer perspective. He constructs other service providers as valuable as well, as they can provide a "different point of view". The following extract, also from Travis' interview, he additionally talks about the benefits for consumers associated with being employed as a peer worker:

Travis, 19/2/09

T: Yeah. This has saved my life. Without this place, I could've been sitting at home feeling frustrated and bored because I can't work properly. This place, I can work, peer work, I can work because they understand that I need certain privileges and attention and then if I need a break, I can have a break because they know that I need a break.

L: Because they understand your needs better.

T: But if I got a full-time job and they said, you're not doing your job well and I said, I'm feeling a bit unwell, you know, they'd say, you're fired, you know what I mean? So yeah, it gives you that chance to find that medium.

According to Travis, being a peer worker employed at a community centre has "saved [his] life". He elaborates that the alternative to working as a peer worker would be a life of unemployment, boredom and frustration. Travis highlights that his workplace allows him certain, necessary flexibility, "privileges and attention" due to understanding of his condition, which is unfortunately, not shared with the general community. He contrasts this with other forms of employment, whereby he would be "fired" for being unable to consistently perform to the required standard due to his illness. 
Thus, it could be argued that in addition to peer workers providing support to other consumers, consumers who undertake peer work may be afforded a greater sense of purpose and satisfaction - not solely because of their roles but because of their workplaces as well.

\subsection{Case managers}

Not all of the consumers interviewed had been allocated case managers (social workers), however, some interviewees talked about the role their case managers have played in assisting their adherence. Of note, some of the extracts related to relationships between consumers and case managers have previously been presented under sub-codes related to valuable components of relationships with service providers. There is little research in relation to the therapeutic alliance between case managers and mental health consumers.

The following extracts highlight the variation in consumer perceptions of case manager intervention, which was described as either intensive and helpful, or non-intensive and unhelpful, for consumer outcomes. In the following extracts, when asked how health workers could assist with medication, Katherine and Margaret position case managers as having a valuable role in assisting adherence. Katherine talks generally about the benefits of case workers monitoring consumers' adherence via regular home visits and Margaret talks about her personal experience of her case manager assisting her to address skipped dosages as a result of forgetfulness:

Katherine, 5/2/09 
L: And so what about with the medication though, do you reckon like that health workers or other people can have a role in helping someone stay on their medication?

K: Um, yeah that works pretty well when people have got community workers and stuff like that. Um, with that intensive um assistance it helps a lot.

L: Aha.

K: Yeah.

L: Yeah so to have someone who will go around to their houses and see them and check up.

K: Yes, that's right and often, if they're really bad, they get, might get two visits a day and they take their medication. So they have some services out there in the community.

Margaret, 4/2/o9

L: Um, do you think health workers could have a role in helping with medication?

M: Definitely. Um there was one time when I would forget my tablet and it was while I was on Fluphenazine, I think. And Kieran, I don't know if you know him, from John St., he's a social worker.

L: Oh ok, yep.

M: He, he was actually part of the mental health team that was assigned to me. I had Jane and then I had Kieran. This was when my psychosis was quite bad at times.

L: So you had like a case manager.

M: Yes. He was my case worker as well as being a social worker. Uh, I had to ring him and I'd say, Kieran, I've forgotten to take my tablet. And he'd 
say, well that's ok. How are you feeling? And I'd tell him. And he'd say, yeah, you're sounding alright. Um, don' take it. Uh leave it until the next-

\section{L: Night?}

M: Yep. Which would be that night you see? Because I would have phoned him early in the morning that I had forgotten. At the time I had the dosettes, the blue dosettes from uh with um, you still get morning, lunch, tea and dinner, but it's not like the Webster pack [medication pack]. The Webster pack's a complete year in it. Where, the dosette, you have to open the drawer and tip them out but if you've got um day time medication or of you open the wrong one, it it's like a slide thing that you slide it open and take which dose.

L: Oh right

M: You need, and then of course it be sat there, in the dose, the medication, I hadn't took it. And he'd guide me through it. You know, how are you feeling? What's been happening? What's been happening to your voices? Are they bad? What's the content? Is the content bad? Um, he'd he'd guide me through that, so I think they're very, very important.

Katherine posits that "community workers" can provide "intensive assistance" which can positively influence adherence and Margaret concurs that health workers can assist with medication ("Definitely") and positions case managers, in particular, as "very, very important". With prompting, Katherine agrees that home visits may assist consumers to remain adherent, which should occur more regularly when consumers are particularly unstable ("if they're really bad, they get, might get two visits a day"). Margaret refers back to when she experienced difficulties remembering to take her medication ("there was one time when I would forget my tablet") when her 
illness symptoms were severe ("my psychosis was quite bad at times"), which her case manager reportedly supported her through. Regarding the type of support her case manager provided, Margaret states that she telephoned him when she missed a dosage and he would ask her questions related to her how she was feeling, any potential illness triggers (“What's been happening?") and her symptoms and symptoms severity ("What's been happening to your voices? Are they bad?”). On the basis of her presentation, the case manager would then provide instructions to Margaret, to support her sustained adherence to her medication routine ("He'd guide me through it"). Below, Amy highlights another role that her case worker has fulfilled; namely, to liaise between her and the psychiatrist to discuss medication issues:

Amy, 10/2/09

L: What has your relationship been like with your case manager?

A: (laughing) Yeah, um got off to a reasonable start um had a few problems, uh, because we're so different and I didn't give her the respect she deserved (inaudible). Um, yeah, I found it, I thought she was being bitchy and nosy and we just had a bit of a personality clash but like mum said, she's like a friend, a professional friend, she's been a good friend like someone who can stick up for me and she's liaised really well with me when I've been having problems from my perspective with my psychiatrist and really not getting through my opinion and my, what I believe my founded fears of the negative impact of medication and the type of medication I was on. 
Amy acknowledges that she and her case worker had a "personality clash" and she "didn't give [case manager] the respect she deserved". Nonetheless, Amy likens her case manager to a "professional friend". She implies that her case manager has assisted her by defending and communicating her perspective to her psychiatrist ("someone who can stick up for me"), particularly in relation to her "fears of the negative impact of medication and the type of medication I was on". Thus, Amy could be seen to suggest that in instances when her psychiatrist may have dismissed her concerns about her medication, her case manager has acknowledged her concerns and attempted to communicate these to the psychiatrist, thereby providing a voice for Amy in relation to her treatment.

Whilst the previous extracts described intensive, consumer-focused case work aimed at preventing non-adherence and relapse, this was not consistent with other consumers' experiences with case workers. In the following extracts, for example, Rachel and Nathan describe their case managers as absent and lacking thoroughness:

Rachel, 25/2/09

L: Yeah. What was your case manager like?

$R:$ Useless. I only ever saw her once every six weeks or spoke to her once every six or seven weeks. She didn't do much at all.

Nathan, 25/2/09

L: How could workers help with adherence?

$N$ : If they come round and actually ask you if you're taking your medication. My workers don't come out and do any of that. They just come out and don't ask me if I've been taking it. 
Rachel describes her case worker as "useless" and elaborates that she "didn't do much at all". Both Rachel and Nathan highlight the limited contact with their case managers. According to Rachel, she only had contact with her case worker "every six or seven weeks". When asked how workers could assist with adherence, Nathan indicates that they could conduct home visits ("come round") and "actually ask if you're taking your medication". He contrasts this with his experience of workers failing to "do any of that". Whilst Nathan later acknowledges that his workers visit, he states that they "don't ask" him about his adherence. These extracts, considered in conjunction with the previous extracts, which described case worker intervention positively, suggest that there are varying degrees of intensity of case work.

\subsection{Chapter summary}

Several service-related factors were commonly raised as influences on adherence in interviewees' talk. The main service-related factors mentioned were the therapeutic alliance between the prescriber and consumer, interactions with case managers and peer workers and experiences of community centres. The therapeutic alliance has been established in the literature as an influence on adherence and, consistent with this, it was frequently discussed by interviewees. Interview data highlighted which components of the therapeutic alliance were considered important to adherence to interviewees. Specifically, interviewees indicated that collaboration and shared decision-making regarding treatment were important predictors of adherence. An authoritative or punitive prescriber 
approach to treatment was generally linked to non-adherence (often as an expression of resistance), however, some interviewees attributed adherence to a fear of the prescriber's response to non-adherence. Interviewees indicated that they wanted their prescribers to ask them relevant questions to assist with their illness and treatment management, which was contrasted with experiences of an impersonal service from prescribers, who forgot simple details including those related to the medication regimen.

Consistent with previous research, experiences of a rotational system of psychiatrists in a medication clinic setting were framed negatively. Specifically, it was reported that such a system compromised consumers' abilities to build relationships with prescribers and to talk openly about issues due to shorter durations of treatment from the same psychiatrist.

The most important element of the therapeutic alliance that was consistently raised in interviewees' adherence talk related to prescribers tailoring the medication regimen to consumers' unique circumstances. Arguably, tailoring also encompasses collaboration and genuine interest in, and knowledge of, consumers, as covered in previous sub-codes. In addition to tailoring the medication regimen to symptom fluctuations and periods of stability, interviewees also stated that it was important for prescribers to take into account their daily routines and to target information according to their intellectual abilities or levels of understanding.

Interviewees consistently described their experiences with case workers and community centres positively. They additionally often supported a greater role for peer workers in assisting with adherence due to their shared experiences. One interviewee also reported an enhanced sense of purpose associated with working as a peer worker, consistent with recovery 
research, which relates consumers' involvement in such programs to empowerment. In particular, recovery research supports people to build and sustain self-defined, purposeful lives and identities, which can be accomplished through roles including peer work (as opposed to clinical recovery, which focuses primarily on symptom reduction and return to premorbid functioning). Constructions of case managers varied significantly. Whilst one interviewee indicated that her case manager communicated her treatment-related concerns to her prescriber and, thus, represented a mediator of sorts, others indicated that their case managers had minimal involvement in their lives. 


\section{Chapter 8: Discussion}

The qualitative research presented in this thesis explored medication adherence amongst a sample of outpatients with schizophrenia who were prescribed antipsychotic medications. As Novick et al. (2010) point out, greater understanding of the factors associated with medication adherence and non-adherence is needed so that any modifiable risk factors can be identified and managed using appropriate interventions. In approaching the study, a qualitative research design that involved interviewing consumers about their experiences with antipsychotic medications made intuitive sense given the research aim was to enhance understanding of medication taking and consumers were considered the experts in their own illness and treatment experiences. Nonetheless, in reading the available literature on the topic area, it was discovered that few qualitative studies have previously been undertaken in the area. Whilst some qualitative research has been conducted involving people with schizophrenia, few studies solely focus on medication adherence. Moreover, qualitative research that relates to medication adherence has often included participants with other psychiatric or chronic illnesses (i.e., Carder et al., 2003; Sharif et al., 2003; Shoemaker \& Ramalho de Oliveira, 2008), thus, the findings are not specific to the population of interest. Therefore, the present research addresses the relative absence of

qualitative research in relation to medication adherence amongst people with schizophrenia.

This research additionally gives voice to consumers with schizophrenia, whose unique perspectives are largely overlooked in adherence research. That is, whilst many quantitative studies have been 
undertaken which typically measure rates of adherence or pre-determined sets of factors to assess for their associations with adherence via surveys, for example, few have actually given participants opportunities to identify and discuss the factors that they think are relevant to their adherence. Whilst some qualitative research has provided some scope for new factors to emerge, interview schedules frequently focused on pre-established factors rather than containing general questions. It has been proposed that neglect of the consumer perspective in previous research may reflect perceptions that people with schizophrenia are irrational and incapable of offering a valid viewpoint (Rogers et al., 1998). By contrast, in the present study, participants represented valuable resources for in-depth information which could inform clinical practice in relation to medication adherence.

\subsection{Interview experiences: support for greater inclusion of the consumer perspective in future research}

My experiences of interviewing study participants provide further evidence that people with diagnoses of schizophrenia, including those who experience ongoing challenges, are not defined solely by their illnesses. That is, they are individuals with unique abilities, strengths, experiences and capacities for growth, just like people without diagnoses of schizophrenia. Interviewees engaged meaningfully with interviews and their voluntary participation could be seen to reflect willingness to contribute to the production of knowledge about schizophrenia and its treatment. Interviewees tolerated my occasional ignorance with patience and respect and provided me with thorough explanations as required. As can be seen from the interview data, 
interviewees offered in-depth, thought-provoking insights into their own situations and experiences and provided valuable, creative opinions regarding how services could be improved to assist with medication adherence and better outcomes for consumers more generally. Whilst interviews were not therapeutic in any way (although I deployed my counselling skills such as active listening and unconditional positive regard) and despite interviewees receiving a relatively minor monetary reward (AU\$10) for their participation which took up to approximately two hours in length, interviewees often thanked me after interviews. Such a response to study participation highlighted to me that consumers with schizophrenia in Adelaide (and possibly more generally) may relish opportunities to offer their perspectives and to feel heard and as though their opinions are valued.

Indeed, feeling as though their views were listened to was frequently raised as an important factor related to the therapeutic alliance with prescribers. As mentioned earlier, this could be because consumers' viewpoints are frequently not taken seriously, or considered invalid, due to the stigma associated with a diagnosis of schizophrenia. These findings also provide support for involving consumers more in research, including allowing consumers to guide the research process, as the recovery model endorses.

\subsection{The sample and generalisability of findings}

The research sample comprised of 25 outpatients with schizophrenia who were affiliated with hospitals, day centres and medication clinics in the metropolitan Adelaide area. The variation in gender, age at time of interview and at diagnosis and medication treatment regimens ensured that despite the relatively small size, the sample was a fair and adequate reflection of the study population, thus, maximising the potential transferability of the study. 
To enhance the rigour of the present study, I used exploratory, open-ended questions, audio recorded interviews, took field notes and memos and constantly checked and re-checked the analyses. Supervisors also provided feedback regarding coding and analysis. Although adherence rates were not measured in the present study, all of the interviewees were able to reflect on past experiences of non-adherence, consistent with literature which reports high rates of non-adherence amongst people with schizophrenia (Lieberman et al., 2005; Moritz et al., 2009; Mitchell, 2007; Weiden et al., 1994;).

\subsection{Reflections on the analysis}

\subsubsection{Open coding}

Analysis of interview data was guided by a grounded theory approach and involved open coding, whereby codes were identified, named and described. Unlike a traditional grounded theory approach, however, a process model or theory of medication adherence was not generated as this was beyond the scope of the thesis. In line with the majority of the background literature, some of the strongest (most prevalent) codes that emerged in the data as influences on adherence were medication effects (including side effects and effectiveness in treating symptoms), insight and the therapeutic alliance. Analysis of interview data highlighted that these codes are complex and multidimensional, thus, they were all divided into sub-codes in the analysis. Data also shed some light on how the effects of medication, insight and the therapeutic alliance may influence adherence amongst consumers, by elucidating consumers' perceptions of the important aspects of these codes. Another strong code that emerged in the data, but that has not been established in the literature, was reflection on experiences, 
whereby consumers indicated that they reflected on past adherence and nonadherence experiences to inform their decisions about present or future adherence. Other codes that emerged in the data, however were less significant (not raised as frequently) included self-medication, forgetfulness, the route of medication administration, storage of medication, peer workers, community centres and case managers.

Another code that emerged less frequently in the data was stigma, however, this code was largely excluded from the analysis (except where extracts relating to it were also relevant to other codes)because direct associations between stigma and adherence behaviour were limited. Nonetheless, it is of note that stigma has been raised as an influence on adherence in the literature previously. For example, in a pilot study involving consumers receiving outpatient and inpatient treatment for acute episodes, the stigma associated with taking medication represented one of the strongest consumer-reported predictors of non-adherence (Hudson et al., 2004). Additionally, in a qualitative interview study, social stigma and fear of being labelled was attributed to treatment non-adherence amongst some consumers (Sharif et al., 2003). Specifically, consumers who were unwilling to identify themselves as psychiatric consumers avoided attending clinics on review dates and frequently missed scheduled appointments. In the present study, one interviewee stated that medication-taking was a constant reminder of his illness, attributing this to his preference for depot administration. This contradicted the finding by Moritz et al. (2009) that consumers were very sensitive to stigma and forced medication and, thus, opposed depot forms of medication due to their associations with community treatment orders. More frequently, interviewees in the present study talked about their experiences of stigma in the community, manifesting as disadvantages in employment and 
social contexts, for example. Although interesting, this was not relevant to the focus of the present study. Interviewees' constructions of medication as "normalising", however, could be seen to reflect internalised stigma associated with their illness diagnoses. Some research indicates that consumers may react to stigma by denying their illnesses and the need for treatment, which all too often leads to poor outcomes (Liberman \& Kopelowicz, 2005), highlighting how stigma may indirectly lead to nonadherence by compromising consumers' insight.

Interviewees also occasionally talked about their hospital-related experiences. Despite representing part of consumers' interactions with services, as many of these extracts were not directly related to adherence, they were either excluded from the analysis or integrated into other codes where relevant. The hospital-related experiences extracts that were excluded primarily reported inadequate number of beds, lengthy waiting periods and failed attempts at voluntary admissions as a result of these. Such experiences could viably be generalised to mental health consumers in metropolitan Adelaide.

\subsubsection{Axial coding}

Following from open coding, which identified codes, axial coding was undertaken, whereby codes were organised into broader categories. Three categories were distinguished, representing broad aspects of the medication experience amongst the sample. These categories were labelled consumerrelated factors, medication-related factors and service-related factors and encompassed codes that were identified in the data. These three categories represent different aspects of the interviewees' experiences with antipsychotic medications. Consumer-related factors encompass the internal 
negotiations and cognitive processes that take place in relation to medication adherence, including awareness, acceptance, acquisition of knowledge, attributions of experiences, reflection, pattern recognition, memory and problem solving. Medication-related factors encompass the effects of medication on body, including side effects and symptom alleviation. Of great importance to interviewees was how the bodily effects of medication impacted on their daily functioning and lives. Service-related factors comprise the interactional aspect of the medication adherence experience, involving communication and negotiation with health professionals, institutions and systems. Researchers should consider all of these aspects of the medication adherence experience when devising interventions. Furthermore, clinicians should consider all of these factors in their interactions with consumers.

Previous studies have organised factors related to adherence in similar ways, however, additional categories are often included, such as illnessrelated factors and social factors. This may reflect different methods of categorising data. It could also be the case that illness-related factors, such as the presence of symptoms, were assessed more frequently in quantitative research. For example, many quantitative adherence studies compared participants in terms of their scores on measures of symptoms, such as the PANSS (i.e., Perkins et al., 2006). Illness-related factors that have been shown to influence adherence in previous research include symptom severity (Lacro et al., 2002); specific delusional beliefs about medication (Barnes, 1997), particularly paranoid delusions (Janssen et al., 2006); more pronounced negative symptoms (Heinrichs et al., 2008; Janssen et al., 2006); more pronounced positive symptoms (Heinrichs et al., 2008); less improvement in positive symptoms when taking medication (Perkins et al., 
2006); and cognitive problems associated with schizophrenia including in relation to language, memory and learning (Heinrichs et al., 2008). The relationship between illness factors and adherence is difficult to establish however, as medication is likely to improve symptoms. Thus, the results of studies that claim associations between symptoms and adherence may actually reflect the effect of medication on illness symptoms (and the influence that this may exert on adherence).

Insight is also commonly categorised as an illness-related factor in other research, however, in the present study, it was categorised as a consumer-related factor. Whilst the present study does not dispute that a lack of insight is one of the symptoms associated with schizophrenia, it is proposed that distinguishing an illness-related lack of insight, with a lack of insight due to a lack of knowledge or experience, for example, is extremely difficult. Indeed, the finding in the present study that insight may be gained from reflecting on experiences challenges the notion that insight amongst people with schizophrenia represents a purely biomedical construct. Factors that have previously been categorised as social factors, such as family support and substance use, were also considered consumer-related factors in the present study. Although it is frequently considered a social problem, substance use, particularly as a form of self-medication, is not necessarily a social practice. Social support was only occasionally raised in the present study in relation to prompting to assist consumers to overcome unintentional non-adherence, so extracts that related to this were suitably presented after those related to forgetfulness, within the consumer-related factors category. 


\subsubsection{Consumer-related factors category}

Consumer-related factors in the research presented here do not refer to demographic factors. Whilst demographic information was gathered in the present study, demographic factors have been tested exhaustively in relation to adherence, particularly in quantitative studies, but no consistent associations have been found (i.e., Fenton et al., 1997; Lacro et al., 2002; Olfson et al., 2000; Yang et al., 2011). Demographic factors were, thus, not statistically assessed for their relevance to adherence, but rather, were gathered to establish rapport with interviewees and described briefly in the Methodology chapter (Chapter 4) to define the study sample.

The category of consumer-related factors comprised of codes relating to consumers' insight into their illness and treatment, their abilities to reflect on past experiences with medication, self-medication, forgetfulness and strategies to overcome forgetfulness, including integrating medication taking into their individual routines and relying on social supports for prompting. There is strong support for insight as an influence on adherence in the literature (Dassa et al., 2010; Fenton et al., 1997; Lacro et al., 2002; Moritz et al., 2009; Perkins et al., 2006), however, there is some variation regarding how insight is defined. Whilst most researchers define insight as awareness of having an illness, others suggest that it is a multidimensional construct which also encapsulates awareness of the consequences of the illness and the need for treatment (Amador et al., 1991; Olfson et al., 2000). Amador et al.'s (1991) conceptualisation of insight is consistent with the findings from the present study, which highlighted that in addition to awareness of the illness (and symptoms), insight regarding the risk of relapse, the chronicity of the illness and the need for ongoing medication treatment represent important influences on adherence. These findings, thus, highlight how insight that 
operates at, and influences, the different stages of the illness experience, including pre-diagnosis, diagnosis, during and after relapses and during the maintenance treatment phase.

Another important consumer-related factor which emerged strongly in interview data related to reflection on past experiences. That is, interviewees frequently reported that reflecting on their past experiences of adherence or non-adherence, and the associated consequences, influenced their decisions to take or discontinue taking their medication. Interviewees could frequently be seen to indicate that reflecting on their experiences also facilitated gains in insight; however, it could also be argued that insight is required in order to be able to reflect on past experiences. Furthermore, the reflection on experiences factor could be similar to the concept of retrospective insight, as raised by Amador et al. (1991), which is distinguished from current insight.

The reflection on experiences factor is novel as it has not yet been established in the literature. In actuality, the notion that negative past experiences of non-adherence can influence consumers to be adherent in future, as part of a learning process, contradicts the results of prospective studies which suggest that the strongest predictor of future non-adherence is recent past non-adherence (i.e., Ascher-Svanum et al., 2006; Novick et al., 2010). It could also be seen to challenge the prevailing view in the healthcare system that significant attempts should be made to avoid relapse amongst consumers. Whilst the findings do not dispute the potentially devastating consequences of symptom relapse for consumers, relapse associated with medication non-adherence may actually represent an experience that consumers can draw on to reinforce to them the benefits of taking medication. 
Some reference to reflection on illness experiences has been made in earlier research, in which insight was conceptualised as a psychological coping mechanism or defence. For example, McGlashan, Levy and Carpenter (1975) proposed a continuum of recovery styles amongst people with schizophrenia, ranging from "integration" to "sealing over". An interview study was conducted, whereby consumers were interviewed 12 months following an acute episode. Interviews were then categorised into integration or sealing over categories. Sealing over was indicated when consumers demonstrated a preference not to think about, and discuss, their psychotic experience during recovery. Those consumers whose accounts were categorised as integration, on the other hand, manifested an interest in psychotic experiences during recovery and were willing to discuss these experiences in an effort to learn more about themselves. Responses from both groups were evaluated and researchers concluded that integrators displayed an awareness of the continuity of their personality before, during and after acute episodes, took responsibility for their symptoms, and were flexible in their thoughts about them. Those who were classified as sealing over tended to resist thinking about their experiences of psychotic episodes and, when confronted by others, were unaware of aspects of it. They additionally viewed psychosis as alien and caused by some force outside themselves. The differences in awareness/insight described by McGlashan, Levy, \& Carpenter (1975) relate to past events (retrospective awareness/insight) and have been interpreted as reflecting coping strategies applicable to other stressful life events besides having schizophrenia (Amador et al., 1991). It could, therefore, be argued that the sealing over and integration coping strategies could also be applied to consumers' experiences with medication (which also relate to illness experiences). Integration 
appears similar to the reflection on experiences code, as in the relevant extracts, interviewees took ownership of, and demonstrated and a willingness to discuss, their illness and medication experiences. Furthermore, they directly stated that reflecting on these experiences facilitated learning.

Wciorka (1988) proposed that consumers' willingness to be reflective about their illness represented a dimension of their attitudes towards their illnesses in addition to identification of the illness with themselves and evaluation of their illness. These results were based on data collected from an interview study in which participants were encouraged to talk about their subjective definitions of their illnesses. The three dimensions were interpreted as reflecting the cognitive, evaluative and reactive components of attitude toward illness (willingness to be reflective representing the reactive component). Thus, results of early research by McGlashan et al. (1985) and Wciorka (1988) could be seen to suggest that reflection on (and interpretation of) illness experiences are aspects of insight or attitudes towards illness, which may explain why this factor has not been raised as a separate influence in more recent adherence research. Even if this is the case, whilst the relationship between insight and adherence has been thoroughly tested, limited attention appears to have been given to the specific relationship between reflecting on past experiences and adherence.

Another possible explanation for the surprising absence of reflection on experiences in previous adherence research could be because previous studies have primarily tended to focus on factors associated with nonadherence rather than adherence. Additionally, previous studies may have failed to ask participants about, or overlooked, the cognitive processes underlying their adherence decisions. For example, rather than considering the process of reflecting on experiences as an influence in itself, researchers 
may have focused on the content of those experiences, such as hospitalisation, and associated the content of specific past experiences with adherence or non-adherence. Another possible explanation for this difference could relate to the nature of the present study sample. Specifically, to reiterate, the sample was comprised of outpatients with schizophrenia, who were predominantly adherent to their present medications. This group of consumers may have had enhanced reflective capacities when compared to first episode consumers, for example, as their symptoms were largely stabilised, most demonstrated insight in relation to their illnesses and the mechanism of medication and, importantly, they had experiences to draw on. Future research could explore whether the reflection on experiences code is replicated by consumers at different stages of their illnesses.

Following on from the insight and reflection on experiences code was the self-medication code. Self-medication in the present context refers to consumers deciding to start, adjust or stop taking prescribed medication according to their perceived health needs (Mitchell, 2007). Interviewees occasionally reported that they self-medicated with their prescribed medication to address symptom fluctuations or kept a store of medication to prolong periods between depot administrations. In relevant extracts, interviewees demonstrated insight in relation to their symptoms and triggers for relapse. Thus, similar to the findings of some previous qualitative research (i.e., Rogers et al., 1998; Shoemaker \& Ramalho de Oliveira, 2008), it may be the case that once consumers have gained experience and knowledge in relation to their illnesses and medications, they are more likely to self-medicate in an attempt to tailor their medication to their circumstances and to assert control over their illness management. Consistent with Mitchell's (2007) hypothesis, decisions to self-medicate then 
are often conducted intentionally and rationally. Some interviewees reported self-medication in collaboration with prescribers, suggesting some acceptance of this practice and flexibility amongst prescribers, despite the challenges that the practice poses to strict definitions of adherence.

Some interviewees additionally reported self-medication with nonprescribed drugs to treat symptoms, however, this practice was not as common as expected given the high rates of comorbid substance use and schizophrenia that are frequently reported (McPhillips et al., 1997; Mueser \& Lewis, 2000). Substance use has consistently been raised as a strong influence on adherence in the literature (Ascher-Svanum et al., 2006; Lacro et al., 2002; Novick et al., 2010; Olfson et al., 2000). Lower reported substance use than expected may have been a reflection of the outpatient, generally adherent sample. Other possible explanations for this could be because interviewees were not routinely directly asked about their substance use or that consumers with co-morbid substance use may have been less likely to volunteer to participate in the present study. The present study's findings could also suggest that self-report is an unreliable means of ascertaining the influence of substance use on adherence, due to interviewees' potential reluctance to admit to substance use to researchers. One interviewee who acknowledged past self-medication with amphetamines also reported treatment resistance, possibly accounted for by his substance use (Shean, 2004).

Forgetfulness was also occasionally raised as an influence on interviewees' adherence, consistent with previous findings (i.e., Moritz et al., 2009). It has been suggested that forgetfulness could be explained by the cognitive deficits associated with schizophrenia, including compromised memory and executive functioning (Moritz et al., 2009). In order to 
overcome forgetfulness (unintentional non-adherence), interviewees reported incorporating medication into their daily routines, such as taking their medication at meal times, and encountered occasional inconveniences associated with having to consider medication in their plans. Interviewees also occasionally reported reliance on social supports, particularly coresidents, for prompting to take their medications, consistent with the findings of a previous qualitative study (Rogers et al., 1998).

To summarise the interactions between consumer-related factors, insight and reflection on experiences appear to be intrinsically linked. Previous research suggests that reflection on experiences may represent an aspect of insight. Nonetheless, interview data suggest that insight can be gained from reflection on experiences via a learning process and many interviewees suggested that prompting consumers to reflect on their nonadherence experiences could assist in reinforcing adherence. It could also be logically argued that insight is required in order to be able to reflect objectively on experiences and make valid attributions. Consistent with previous research, once consumers gain insight in relation to their illnesses and their medication, they may be more inclined to self-medicate. Consumers may draw on past experiences to evaluate when it is appropriate to self-medicate. Interviewees also reported self-medication in collaboration with prescribers. Thus, rather than reflecting resistance, self-medication in this context reflects consumers tailoring their treatment to their illnesses and their specific circumstances, accounting for symptom fluctuations and potential triggers. Whilst forgetfulness appears disconnected from the other codes, it still represents a consumer-related factor and may relate to memory and executive functioning deficits associated with schizophrenia. To an extent, consumers' strategies to overcome forgetfulness may reflect tailoring 
to their personal circumstances. They also involve problem solving on behalf of consumers. Occasional forgetfulness (unintentional non-adherence) should be normalised taking into consideration the additional cognitive demands of having to consider medication in all daily undertakings. This would be a large responsibility for anyone to take on, let alone those who may suffer cognitive deficits.

\subsubsection{Medication-related factors category}

The medication-related factors category comprised of codes related to the route of administration, storage of medication, side effects and efficacy of medication. All of these factors have previously been raised in the literature, with particularly strong support for side effects and efficacy influencing adherence, and except for the storage of medication code. Specifically, interviewees indicated that storing their medication in packs which organise medication into doses required each day, assisted them to overcome unintentional non-adherence. Regarding the route of administration, only limited support was found for depot injections being preferable as consumers were not required to take their medication as frequently. Notably, the route of administration and the storage of medication codes were relatively minor (infrequently raised) and did not appear to interact strongly with the major codes central to the medication-related factors category: side effects and the efficacy (or inefficacy) of medication in treating symptoms.

Consistent with the findings of previous qualitative research (Carrick et al., 2004; Rogers et al., 1998; Shoemaker \& Ramalho de Oliveira, 2008), interviewees frequently reported that they weighed up side effects and efficacy in symptom alleviation when making adherence-related decisions. This finding could be seen to support research which indicates that side 
effects and symptom alleviation often occur concurrently (Moritz et al., 2009; Sharif et al., 2003). Moreover, also consistent with previous findings (Rogers et al., 1998; Shoemaker \& Ramalho de Oliveira, 2008), the ways in which side effects and illness symptoms impacted on interviewees' lives was constructed as central to their adherence choices. For example, when illness symptoms or side effects inhibited interviewees' opportunities and social interactions, nonadherence was typically indicated. Thus, medication was constructed as either enabling or disabling consumers from being able to lead productive, satisfying, "normal" lives. This again highlights how consumers may rationally approach the decision to take medication and are willing to take a medication once they believe that the medication's benefits outweigh its risks (Shoemaker \& Ramalho de Oliveira, 2008). Such results highlight the importance of finding a suitable medication regimen for consumers that effectively treats symptoms and simultaneously has a tolerable side effect profile (Resnick et al., 2004).

Despite not reflecting a unique code or sub-code in the analysis, as relevant extracts were divided amongst other codes, interviewees frequently indicated that finding the right medication or combination of medications and the appropriate dosage/s is often a long process, consistent with previous research (Carder et al., 2003). This process was described as challenging adherence as interviewees reported disillusionment and confusion when medications failed to work and/or created significant side effects. Although covered somewhat in the service-related factors category, and also consistent with previous research (Carder et al., 2003), the perspective of being a human experiment was shared by several interviewees. The experimentation period referred to may be difficult to overcome, as prescribers cannot predict how a consumer will respond to a medication. As was made apparent in the 
service-related factors category, however, prescribers could modify their practice in order to create more equality in the therapeutic alliance which might enable consumers to feel as though they have more control over their treatment.

\subsubsection{Service-related factors category}

The service-related factors category included the therapeutic alliance code as well as codes related to interviewees' experiences with services and other staff, such as day centres, peer workers and case managers. The most prominent service-related factor to emerge from the data was the therapeutic alliance. Whilst this factor has already been established as a strong predictor of adherence in the literature (i.e., Dassa et al., 2010; Olfson et al., 2000), categorisation of extracts into sub-codes helped to shed some light on the specific aspects of the therapeutic alliance that are deemed important to adherence and generally, by interviewees. Specifically, interviewees indicated that the power relations within the therapeutic alliance were important and generally associated collaboration with enhanced adherence and authoritarianism with non-adherence; however, exceptions existed. For example, some interviewees attributed their adherence to prescribers' threats of punishment for non-adherence. Interviewees also highlighted the importance of prescribers demonstrating genuine interest in the consumer experience and their backgrounds. Specifically, interviewees suggested that prescribers should ask more in-depth questions and should demonstrate knowledge of consumers' histories. The latter was reportedly contraindicated by rotating staff systems at medication clinics, which mean that consumers often see multiple different prescribers, compromising their abilities to establish meaningful relationships with prescribers. Interview data 
overwhelmingly suggested that tailoring the medication schedule to consumers' unique situations is the most important element of the therapeutic alliance and exerts a significant influence on adherence.

Tailoring to consumers' symptom fluctuations and their experiences of medication effects was deemed important, in addition to tailoring to consumers' lifestyles, personalities and cognitive abilities. It could be argued that in order for prescribers to be able to tailor treatment effectively to individual consumers, they need to act collaboratively, require knowledge of the consumer, need to ask appropriate questions to guide the tailoring process and, ideally, there would be some continuity of the therapeutic alliance to facilitate in-depth understanding of the consumer, their illness and their responsiveness to medication. Some interviewees also admitted to non-adherence to express resistance to prescribers or to elicit some attention from health professionals who failed to act on concerns raised by consumers. This finding highlights the importance of a positive therapeutic alliance in influencing adherence, as the relationship dynamic is constructed as the primary reason for non-adherence, outweighing other important medicationrelated factors or consumer-related factors.

Although not a major code to emerge, several interviewees indicated that specialised services provided by community centres for people with mental illnesses, can positively influence adherence. This finding is consistent with my impressions of community centres prior to and during data collection. Although not reflected in the analysis, interviewees appraised community centres positively in general (not just in relation to adherence) as they reportedly provide a social outlet for consumers, information and a sense of purpose for some. Moreover, interviewees commonly advised that peer workers (who are frequently employed by 
community centres) could have a greater role in assisting consumers with adherence. Peer workers were frequently positioned as more relatable and credible than other health professionals, who lacked direct experience of having a mental illness and taking antipsychotic medication. The role of peer workers in promoting medication adherence has not previously been explored extensively (if at all) and, thus, may represent an avenue for future research. Additionally, peer workers involved in the present study reported that their experiences in this role were positive and empowering, consistent with recovery literature, which suggests that participation in consumer-run programs may be instrumental in motivating a person to sustain treatment and rehabilitation (Liberman \& Kopelowicz, 2005). Evaluations of case managers, by contrast, were mixed, with some interviewees indicating that they were helpful whereas others considered this service relatively hands-off and ineffectual.

Summarising the service-related code reveals several limitations to the current services available for consumers in metropolitan Adelaide (and potentially beyond). Despite the therapeutic alliance having been established consistently as a strong predictor of adherence, there remain rotating systems of psychiatrist in medication clinics. Additionally, whilst there has been an ideological shift in conceptualisations of the relationship between health professionals and consumers towards greater equality and enhanced agency and control of the consumer, this does not appear to reflect practice, in the treatment of schizophrenia, at least. Too frequently, interviewees reported power imbalances in favour of the prescriber in therapeutic alliances. Tailoring to consumers' unique circumstances often does not take place and there appears to be a concerning lack of interest in the consumer perspective, reflected through a lack of knowledge of consumers' histories and repetitive, 
concrete questioning. These results challenge how "therapeutic" the relationship between the prescriber and the consumer actually is, especially in the context of non-adherence to express resistance, whereby interviewees reported taking extreme measures such as over-dosing or becoming nonadherent to induce a psychotic episode in order to elicit responses from prescribers or the healthcare system. Experiences with case managers were mixed and, notably, few interviewees actually had case managers. One interviewee positioned her case manager as similar to a consumer advocate, who facilitated communication between herself and her prescriber, highlighting how case managers could have pivotal roles in assisting with adherence and improving relationships between prescribers and consumers. Positively, there was support for the increased role of peer workers in services to assist with adherence, who were consistently positioned as credible and relatable, highlighting a potential clinical implication of the present research.

As can be seen, there is significant overlap and interaction between the categories outlined. Whilst they have been divided up for presentation purposes, they should be considered collectively, particularly when interventions and clinical implications are being considered. A possible reason for the ineffectiveness of previous interventions could be that they have focused on only one or a few factors or categories and, thus, failed to address other important aspects of medication taking experiences.

\subsection{Potential clinical implications}

The present study originally set out to devise an intervention to assist consumers with their medication adherence on the basis of interview findings. Several interventions have been proposed to address adherence, 
however, a review of the literature indicates that they have had limited effectiveness, with only approximately one third of interventions leading to significant improvements in adherence (Zygmunt, Olfson, Boyer \& Mechanic, 2002). Interventions that have most commonly been studied include family therapy, psychoeducation, behavioural and cognitive therapies. Neither family therapy nor psychoeducation have consistently been associated with improvements in adherence, unless combined with other therapies/intervention modalities (Eckman \& Liberman, 1990; Fenton et al., 1997; Gray et al., 2002; Haynes, Ackloo, Sahota, McDonald \& Yao., 2008; Kelly \& Scott, 1990; MacPherson et al., 1996; Smith et al., 1992; Xiang et al., 1994; Xiong et al., 1994; Zygmunt et al., 2002). When psychoeducation has been shown to moderately improve adherence, the effect has tended to be modest and not sustained over time (Lowe, Raynor, Courtney, Purvis \& Teale, 1995). Behavioural interventions have been shown to be comparatively more effective at improving adherence than other modalities (Boczkowski, Zeichner \& DeSanto 1985; Falloon, 1984; Zygmunt et al., 2002), possibly because they are typically based on learning principles that are generalisable to most people and because they were frequently tailored to the individual situations of consumers. Cognitive and motivational interviewing interventions have also been studied, to a lesser extent than other interventions, with mixed results (Gray et al., 2002; Kemp, Kirov, Everitt, Hayward \& David 1998; O’Donnell et al., 2003).

In the present study, interview data overwhelmingly contraindicated the utility of a generalised, "one size fits all", pre-determined intervention to address adherence amongst consumers. This finding could explain why previous intervention studies have reported only limited success in terms of improving adherence. Interviewees in the present study justified their 
opposition to such interventions through constructions of adherence as a personal issue, influenced largely by consumers' unique experiences. This is consistent with the findings of other qualitative research (Carrick et al., 2004; Shoemaker \& Ramalho de Oliveira, 2008), in which interviewees conveyed that they are the ultimate managers of their treatments and create their own medication practices. Adherence was frequently described as a process which involves experiential learning, and is thus, mediated by a variety of factors at different stages of the illness, which differ for individual consumers. Adherence could, thus, be seen to encompass a complex interaction of influences, which may change over time. Furthermore, adherence decisions were frequently framed as rational choices based on the information and resources available to consumers. Interviewees acknowledged that in some instances, non-adherence not only represents a logical choice, but it may be the best option for consumers, contrary to assumptions in the literature (and arguably the healthcare setting) that adherence is always positive and should be strived for. Additionally, adherence and non-adherence experiences, including associated consequences, were often constructed as learning opportunities. Many interviewees reported that non-adherence experiences, in particular, reinforced the benefits of taking medication. Similarly, Carder et al. (2003) pointed out that consumer resistance and acceptance of taking medication represent dimensions of the negotiation process involved in adherence, yet neither is final. Furthermore, they posited that resistance (or non-adherence) may allow consumers to actively question and participate in their treatment plans, in line with the present findings.

Results of the present study, therefore, highlight the limited effectiveness of prescribers and other health workers imposing or encouraging adherence amongst consumers who do not themselves perceive 
adherence as desirable or appropriate. Indeed, this was frequently articulated by interviewees. For example, interviewees indicated that when medications failed to treat symptoms or produced intolerable side effects, adherence is neither an attractive nor a beneficial option. A more extreme example of the bodily effects of medication rendering adherence unsuitable is treatment-resistant schizophrenia. Prescriber intervention to encourage adherence in such instances was generally seen to reflect failure to take into account the consumer experience and, according to consumers, can result in non-adherence as resistance. Interviewees proposed that a more effective prescriber approach may involve asking about and acting on the consumers' concerns about the medication schedule and addressing concerns, by changing medications, for example. Similarly, for consumers who lack insight in relation to their illness and/or the need for medication, nonadherence may also typically represent a logical choice. Interviewees suggested that experiencing the consequences of non-adherence can actually be beneficial when there is a lack of insight, as it highlights the need for medication and can provide a reference point to motivate future adherence. It could be argued that peer workers, whose role in assisting with adherence was largely endorsed by interviewees, may also have a useful role in encouraging adherence amongst consumers who lack insight. Specifically, interviewees indicated that by sharing their personal illness and medication experiences, peer workers could highlight the benefits of medication to consumers, potentially protecting consumers from experiencing the negative consequences of non-adherence themselves in order to recognise its benefits in terms of stabilisation.

Based on interview data, it is thereby proposed that there needs to be greater acceptance of non-adherence in the mental health field. Indeed, 
interview data could be seen to suggest that non-adherence should be expected in many cases, such as upon diagnosis, when consumers may discontinue their medication to test their diagnoses due to a lack of illness awareness. Furthermore, dominant perceptions of non-adherence as reflecting resistance need to be re-framed, as the present findings suggest that non-adherence can reflect a rational choice for consumers. Partial adherence via self-medication practices may also reflect a rational choice for consumers, as interview data indicated that upon gaining knowledge and experience of their illnesses and medication, consumers may then tailor their medication schedule to their circumstances, accounting for symptom fluctuations and delayed depot administration, for example. Greater tolerance of non-adherence would inevitably require enhanced understanding of, and empathy towards, the consumer perspective amongst service providers.

Although the present study findings suggest that adherence is largely an individual process which cannot be imposed, there remains a critical role for service providers in helping to guide consumers through negotiations, helping them to identify the pros and cons of different choices, how to best self-regulate and the effects that symptoms, side effects and medication use have on the body and the self-identity (Carder et al., 2003). Regardless of who is involved, any form of clinical intervention aimed at improving adherence should be tailored to the unique circumstances of consumers, as emphasised overwhelmingly by interview data. Interview data suggest that prescribers and other service providers (i.e., case managers) do not always ask consumers enough in-depth questions about their illness, medication and life experiences, which may compromise their abilities to provide a personalised, tailored service and, more specifically, may hinder their 
understanding of consumers' reasons for adherence and non-adherence. As has been illustrated, tailoring may include enhanced tolerance of nonadherence and targeted intervention addressing the unique reasons for nonadherence. Additionally, interviewees indicated that prescribers should also take into consideration non-illness or medication related factors, such as daily routines and cognitive abilities, when delivering information and prescribing medication. When considering consumers' responses to medication, interview data suggest that it may be beneficial for prescribers to consider the impact of side effects and effectiveness on consumers' lives and their daily functioning, as opposed to adopting a purely biomedical perspective. Similarly, previous researchers have reported that service providers need to recognise not only the physical side effects of medications, but the emotional ones as well (Carder et al., 2003).

Furthermore, interview data supported an increased role for peer workers in assisting with adherence and helping consumers to manage their illnesses and treatment more generally. As previously mentioned, peer workers were frequently constructed as reliable, credible sources of support and information for consumers, due to their shared experiences. They were also occasionally positioned as sources of inspiration for consumers struggling with their illness and adherence. Consistently, literature has suggested that consumers who are living well act as positive role models for other consumers and service providers and have a heightened capacity for empathy and developing relationships with other consumers due to their experiences (Copeland, 2006; Glover, 2005). Furthermore, the peer workers who were interviewed reported enhanced satisfaction and empowerment associated with their roles. This phenomenon has been explored historically and was described as the "helper therapy principle" (Riessman, 1965). 
Simply, Riessman observed that the act of helping another heals the helper more than the person helped. The principle posits that by taking on socially valued roles, consumers are no longer bound to the passive role of a "patient", who is reliant on expert advice. Rather, they serve as role models, who provide feedback and assistance to others, which can have a positive impact on self-concepts and lives. The principle was noted in some 1970 s psychiatry journal articles which reported that helping others was beneficial for helpers in a variety of contexts, such as tutoring for younger children. Interest in the principle has been rekindled as part of the recovery movement. According to Lawn, Smith, \& Hunter (2008, p. 499), "peers working alongside, within and across mental health services provide the vehicle for a recovery orientation to be realised".

There is an international trend towards greater involvement of mental health consumers to support fellow consumers as part of the shift towards more recovery-based services (Lawn, Smith \& Hunter, 2008), however, it is uncertain whether this is purely an ideological shift yet to have translated into practice and research. The peer workers involved in the present study were employed by community centres. Common forms of peer support identified in the literature include naturally occurring mutual support programs (such as groups run at community centres whereby consumers can share experiences), consumer-run programs, and employment of consumers as providers within clinical and rehabilitative settings (Davidson et al., 1999). Peer workers could feasibly have a role in promoting open dialogue about adherence and non-adherence experiences, as well as strategies to assist with adherence. Whilst such dialogue may not necessarily translate into positive outcomes in terms of improved adherence rates amongst consumers, it may prepare consumers for adherence and non-adherence experiences, 
potentially rendering them better equipped to manage adverse responses. Peer workers could also potentially facilitate communication between service providers and consumers and may have even a role in assisting service providers to gain empathy for consumers. Whilst the present findings support the benefits of peer worker services to consumers, particularly in relation to adherence, further exploration of the role that peer workers could have in assisting with adherence is required, particularly given that there is an absence of research in this specific area.

\subsection{Limitations}

It is important to highlight that my personal worldview informed the approach to the study, the data collected and the results presented. In particular, although the present study's interview schedule contained broad, general questions, it is acknowledged that these questions may have facilitated the emergence of some codes. For example, interviewees were asked specifically about their experiences of the benefits of medication, which tended to elicit talk related to the effectiveness of medication in treating symptoms and avoidance of relapse and hospitalisation. Furthermore, my interview style and my interpretation of information provided by interviewees guided the interview process. Additionally, whilst the analysis attempted to remain as close to the interview data as possible, the coding and selection of extracts as well as the analysis inevitably involved subjective interpretation and, thus, other interpretations may also exist concurrently.

There are also several limitations in relation to the transferability of the results of the present study to other populations, particularly in relation to the service-related factors. This is because the service model differs 
between states and territories within Australia, as well as internationally. Furthermore, it is highly likely that consumers' experiences of services in rural South Australia may differ from those of consumers in urban areas. Additionally, the results of the present study may only be relevant to outpatients. More qualitative research needs to be undertaken in various contexts similar to, and different from, the present research that involves participants similar to, and different from, those involved in the present research in order enhance understanding about the factors that influence medication adherence and how these factors do so, and interact with one another.

\subsection{Considerations for future research}

Study findings provided support for greater consumer involvement in research, as is endorsed by the recovery model. Schneider (2010), for example, successfully conducted participatory action research involving consumers with schizophrenia to explore issues affecting their lives including housing and interactions with healthcare staff. Participatory action research (PAR) in the mental health field is based on reflection, data collection, and actions that aim to improve health and reduce health inequities through involving the people who, in turn, take actions to improve their own health (Baum, MacDougall \& Smith, 2006). Thus, in studies adopting a PAR methodology, researchers typically work in collaboration with individuals or communities in a manner that leads to action for change. PAR challenges the nature of knowledge and the extent to which knowledge can represent the interests of the powerful and serve to reinforce their positions in society. It additionally affirms that experience can be a basis of knowing and that experiential learning can lead to a legitimate form of knowledge that influences practice (Baum et al., 2006). Although consumers provided the data for the present study, future research in the area could 
benefit from involving consumers in all processes of research, including data collection, analysis, literature reviews and the identification of research questions. Peer workers, for example, could take on leadership roles in adherence research. Consumers may be more receptive to research that involves peer workers given that in the present study, interviewees frequently positioned peer workers as more relatable and more credible sources of information than healthcare professionals, who lacked experience with medication. Additionally, peer workers may be better equipped to interview consumers as they may ask more relevant questions due to their shared experiences, which may also lead to more open communication between the interviewer and interviewee.

\subsection{Contribution and significance}

The present study findings highlight that consumers with schizophrenia can, and do, provide valuable contributions to our understanding of medication adherence and lend support for greater inclusion of the consumer perspective in research, potentially throughout the research process. Consistent with previous findings (i.e., Rogers et al., 1998), interview data highlighted that medication adherence is not an all-or-none phenomenon or a simple "yes" or "no" category. Rather, it was constructed as a process, central to which is experiential learning, highlighting the benefits of both adherence and non-adherence experiences for consumers. Results are consistent with previous findings: Adherence is related to factors including insight, side effects and the therapeutic alliance; however, as expected, adherence is a complex phenomenon, influenced by additional factors, which may change over time. Amongst these additional factors were the reflection on experiences and peer worker codes, which have not previously been established as separate influences on adherence in the literature. In most cases, the reasons for adherence and non-adherence were 
linked to multiple factors rather than one specific cause, providing support for service providers to tailor treatment to consumers and contraindicating the effectiveness of generalised interventions. Whilst the benefits of adherence are not disputed, it is proposed that greater acceptance of nonadherence in the healthcare setting is required. Additionally, peer workers appear to have a positive influence on consumers and may be able to play important roles in assisting with adherence, however, further exploration of what peer support might entail is required. 


\section{References}

Amador, X.F., Strauss, D.H., Yale, S.A. \& Gorman, J.M. (1991). Awareness of illness in schizophrenia. Schizophrenia Bulletin, 17(1), 113-132.

American Psychiatric Association (2000). Diagnostic and Statistical Manual of Mental Disorders (Revised 4th ed.). Washington, DC: Author.

Anthony, W.A. (1993). Recovery from mental illness: the guiding vision of the mental health service system in the 1990s. Psychosocial Rehabilitation Journal, 16(4), 11-23.

Ascher-Svanum, H., Zhu, B., Faries, D., Lacro, J.P. \& Dolder, C.R. (2006). A prospective study of risk factors for nonadherence with antipsychotic medication in the treatment of schizophrenia. Journal of Clinical Psychiatry, 67, 1114-1123

Australian Institute of Health and Welfare (2011). The health and welfare of Australia's Aboriginal and Torres Strait Islander people: an overview. Canberra: Australian Institute of Health and Welfare.

Baldessarini, R. (1994). Enhancing treatment with psychotropic medicines. Bulletin of the Menninger Clinic, 58(2), 224-241.

Barnes, T.R.E., Andrews, D. \& Awad, A.G. (2000). Poor compliance with treatment in people with schizophrenia: causes and management. In P.F. Buckley \& J.L. Waddington (Eds.), Schizophrenia and Mood Disorders: the New Drug Therapies in Clinical Practice (pp. 317-329). Oxford: Reed Educational and Professional Publishing Ltd.

Barnes, T.R.E., McPhillips, M.A., Hillier, R., Purl, B.K. \& Joyce, E. (1997). Compliance with maintenance medication in first-episode schizophrenia. Schizophrenia Research, 24(1), p205.

Battaglia, J. (2001). Compliance with treatment in schizophrenia. Retrieved from: http://cme.medscape.com/viewarticle/418612

Baum, F., MacDougall, C. \& Smith, D. (2006). Participatory action research. Journal of Epidemiology and Community Health, 6o(10), 854-57.

Beck, A.T., Rector, N.A., Stolar, N. \& Grant, P. (2009). Schizophrenia: Cognitive Theory, Research and Therapy. New York: Guilford Press.

Birchwood, M. \& Jackson, C. (2001). Schizophrenia. Hove, East Sussex: Psychology Press. 
Blashki, G., Keks, N., Stocky, A. \& Hocking, B. (2004). Managing schizophrenia in general practice. Australasian Family Physician. 33(4), 221-27.

Bloor, M. \& Wood, F. (2006). Keywords in Qualitative Methods: A Vocabulary of Research Concepts. London: Sage Publications.

Boczkowski, J., Zeichner, A. \& DeSanto, N. (1985). Neuroleptic compliance among chronic schizophrenia out-patients: an intervention outcome report. Consulting Clinical Psychology, 53(5), 666-671.

Buchanan, R. \& McKenna, P. (2000). Clozapine: clinical use and experience. In P.F. Buckley \& J.L. Waddington (Eds.) Schizophrenia and Mood Disorders: the New Drug Therapies in Clinical Practice (pp. 21-31). Oxford: Reed Educational and Professional Publishing Ltd.

Buchanan, R.W., Kreyenbuhl, J., Kelly, D.L., Noel, J.M., Boggs, D.L., Fischer, B.A., ... Keller, W. (2010). The 2009 schizophrenia PORT psychopharmacological treatment recommendations and summary statements. Schizophrenia Bulletin, 36(1), 71-93.

Budd, R.J., Hughes, I.T.C. \& Smith, J.A. (1996). Health beliefs and compliance with anti-psychotic medication. British Journal of Clinical Psychology, 35 (3), 393-397.

Carder, P.C., Vuckovic, N. \& Green, C.A. (2003). Negotiating medications: patient perceptions of long-term medication use. Journal of Clinical Pharmacy and Therapeutics, 28(5), 209-217.

Carrick, R., Mitchell, A., Powell, R. A. \& Lloyd, K. (2004). The quest for wellbeing: A qualitative study of the experience of taking antipsychotic medication. Psychology and Psychotherapy, 77(1), 19-33.

Charmaz, K. (2003). Grounded theory: Objectivist and constructivist methods. In N.K. Denzin \& Y.S. Lincoln (Eds.), Strategies of Qualitative Inquiry (pp.203-243). California: Sage.

Compton, M.T. (2007). Treatment non-adherence among individuals with schizophrenia: risk factors and strategies for improvement. Retrieved from URL: http://alignmap.com/2006/o5/18/cme-treatment-nonadherenceamong-individuals-with-schizophrenia-risk-factors-and-strategies-forimprovement/

Conley, R. (2000). Future directions in novel antipsychotics. In P.F. Buckley \& J.L. Waddington, (Eds.). Schizophrenia and Mood Disorders: The New Drug Therapies in Clinical Practice (pp. 59-63). Oxford: Reed Educational and Professional Publishing Ltd.

Copeland, M. E. (2006). Wellness recovery action plan and peer support. Retrieved from URL: http://www.mentalhealthrecovery.com/. 
Corrigan, P.W., Liberman, R.P. \& Engel, J.D. (1990). From non-compliance to collaboration in the treatment of schizophrenia. Hospital and Community Psychiatry 41(11), 1203-1211.

Cramer, J.A. (2008). Medication compliance and persistence: terminology and definitions. Value Health, 11(1), 44-7.

Cramer, J.A. \& Rosenheck, R. (1998). Compliance with medication regimes for mental and physical disorders. Psychiatric Services, 49(2), 196-201.

Cuffel, B.J. (1996). Comorbid substance use disorder: prevalence, patterns of use and course. In R.E. Drake \& K.T. Mueser (Eds.) Dual Diagnosis of Major Mental Illness and Substance Abuse Disorder II: Recent Research and Clinical Implications. New Directions for Mental Health Services (pp. 6577). San Francisco: Jossey-Bass

Cutting, J. (2003). Descriptive psychopathology. In S.R. Hirsch \& D.Y. Weinberger (Eds.) Schizophrenia. Blackwell Publishing: Massachussets.

Dassa, D., Boyer, L., Benoit, M. Bourcet, S., Raymondet, P. \& Bottai, T. (2010). Factors associated with medication non-adherence in patients suffering from schizophrenia: a cross-sectional study in a universal coverage health-care system. Australian and New Zealand Journal of Psychiatry, 44(10), 921-28.

David, A. (1990). Insight and psychosis. British Journal of Psychiatry, 156 (6), 798-908.

David, A.S. \& Adams, C. (2001). Depot antipsychotic medication in the treatment of patients with schizophrenia: (1) Meta-review; (2) Patient and nurse attitudes. Health Technology Assessment, 5(34), 1-67

Davidson, L., Clinman, M., Kloos, B., Weingarten, R., Stayner, D. \& Tebes, J.K. (1999). Peer support among individuals with severe mental illness: A review of the evidence. Clinical Psychology: Science and Practice, 6(2), 165187.

Day, J.C., Bentall, R.P., Roberts, C., Randall, F., Rogers, A., Cattell, D., ...Power, C. (2005). Attitudes towards antipsychotic medication: the impact of clinical variables and relationships with health professionals. Archives of General Psychiatry, 62(7) 717-724.

Eckman, T.A. \& Liberman, R.P. (1990). A large-scale field test of a medication management skills training program for people with schizophrenia. Psychosocial Rehabilitation Journal,13(3) 31-35.

Ezzy, D. (2002). Qualitative Analysis: Practice and Innovation. New South Wales: Allen \& Unwin 
Falloon, I.R.H. (1984). Developing and maintaining adherence to long term drug taking regimes. Schizophrenia Bulletin, 10(3), 412-417.

Fenton, W.S., Blyler, C.R. \& Heinssen, R.K. (1997). Determinants of medication compliance in schizophrenia: Empirical and clinical findings. Schizophrenia Bulletin, 23(4), 637-51.

Frank, A.F. \& Gunderson, J.G. (1990). The role of the therapeutic alliance in the treatment of schizophrenia: Relationship to course and outcome. Archives of General Psychiatry 47(3), 228-236.

Freedman, R. (2005). The choice of antipsychotic drugs for schizophrenia. The New England Journal of Medicine (Editorials), 353(12), 1286-1288.

Fylan, F. (2005). Semi-structured interviewing. In J. Miles \& P. Gilbert (Eds.) A handbook of research methods for clinical and health psychology (pp.65-79). Oxford: Oxford University Press.

Geller, J.L. (2000) Personal accounts: First-person accounts in the journal's second 25 years. Psychiatric Services, 51(6), 713-716.

Glanz, K., Rimer, B.K. \& Lewis, F.M. (2002). Health Behavior and Health Education. Theory, Research and Practice. San Fransisco: Wiley \& Sons.

Glanz, K., Marcus Lewis, F. \& Rimer, B.K. (1997). Theory at a Glance: A Guide for Health Promotion Practice. U.S Department of Health and Human Services: National Institutes of Health

Glaser, B.G. \& Strauss, A.L. (1967). The Discovery of Grounded Theory: Strategies for Qualitative Research. Chicago: Aldine Publishing Company.

Glover, H. (2005). Recovery based service delivery: Are we ready to transform the works into a paradigm shift? Australian e-Journal for the Advancement of Mental Health, 4(3), 1 - 4.

Gray, R., Rofail, D., Allen, J., Newey, T. (2005). A survey of patient satisfaction with and subjective experiences of treatment with antipsychotic medication. Journal of Advanced Nursing, 52(1), 31-37.

Gray, R., Wykes, T. \& Gournay, K. (2002). From compliance to concordance: a review of the literature on interventions to enhance compliance with antipsychotic medication. Journal of Psychiatric and Mental Health Nursing, 9(3), 277-84.

Green, A. (2005). Schizophrenia and comorbid substance use disorder: effects of antipsychotics. Journal of Clinical Psychiatry. 66 (Suppl 6), 21-6.

Haas, G.L., Garratt, L.S. \& Sweeney, J.A. (1998). Delay to first antipsychotic medication in schizophrenia: impact on symptomatology and clinical course of illness. Journal of Psychiatric Research, 32(3-4), 151-59. 
Haddad, P.M. \& Sharma, S.G. (2007). Adverse effects of atypical antipsychotics: differential risk and clinical implications. Central Nervous System Drugs, 21(11), 911-936.

Harding, C.M., Zubin, J. \& Strauss, J.S. (1992). Chronicity in schizophrenia: revisited. The British Journal of Psychiatry, 161(Supplement 18), 27-37.

Haynes, R.B., Ackloo, E., Sahota, N., McDonald, H.P. \& Yao, X. (2008). Interventions for enhancing medication adherence (Review). The Cochrane Library (2), Wiley.

Hegarty, J.D., Baldessarini, R.J., Tohen, M., Waternaux, C. \& Oepen, G.. (1994). One hundred years of schizophrenia: a meta-analysis of the outcome literature. American Journal of Psychiatry 151, 1409-16.

Heinrichs, R.W., Goldberg, J.O., Miles, A.A. \& McDermid,Vaz S. (2008). Predictors of medication competence in schizophrenia patients. Psychiatry Research 157(1-3), pp. 47-52.

Heres, S., Davis, J., Maino, K., Jetzinger, E., Kissling, W. \& Leucht, S. (2006). Why olanzapine beats risperidone, risperidone beats quetiapine and quetiapine beats olanzapine: An exploratory analysis of head-to-head comparison studies of second generation antipsychotics. American Journal of Psychiatry, 163(2), 185-194.

Hesse-Biber, S.N. \& Leavy, P. (2004). Approaches to Qualitative Research: A Reader on Theory and Practice. New York: Oxford University Press.

Hesse-Biber, S.N. \& Leavy, P. (2006). The practice of qualitative research. California: Sage.

Heyscue, B.E., Levin, G.M. \& Merrick, J.P. (1998). Compliance with depot antipsychotic medication by patients attending outpatient clinics. Psychiatric Services, 49(9) 1232-1234.

Horne, R., Weinman, J., Barber, N., Elliot, R. \& Morgan, M. (2005). Concordance, adherence and compliance in medication taking: Report for the National Coordinating Centre for NHS Service Delivery and Organisation. United Kingdom: NCCSDO

Hudson, T.J., Owen, R.R., Thrush, C.R., Han, X., Pyne, J.M. \& Sullivan, G. (2004). A pilot study of barriers to medication adherence in schizophrenia. Journal of Clinical Psychiatry, 65(2), 211-16.

Irwin, D.S., Witzel, W.D. \& Morgan, D.W. (1971). Phenothiazine intake and staff attitudes. American Journal of Psychiatry 127, 1631-1635.

Janssen, B., Gaebel, W., Haerter, M., Komaharadi, F., Lindel, B. \& Weinmann, S. (2006). Evaluation of factors influencing medication 
compliance in inpatient treatment of psychotic disorders.

Psychopharmacology, 187(2) 229-236.

Johnson, D.A.W. (1993). Depot neuroleptics. In T.R.E. Barnes (Ed), Antipsychotic Drugs and their Side Effects (p 205-212). London: Academic Press

Jones, P.B. \& Buckley, P.F. (2006). In Clinical Practice Series:

Schizophrenia. London: Elsevier Ltd.

Jones, S.R., Torres, V. \& Arminio, J. (2006). Negotiating the Complexities of Qualitative Research in Higher Education. New York: Routledge.

Julius, R.J., Novitsky, M.A. \& Dubin, W.R. (2009). Medication adherence: a review of the literature and implications for clinical practice. Journal of Psychiatric Practice, 15(1), 33-44.

Kane, J.M. \& Borstein, M. (1985). Compliance in the long-term treatment of schizophrenia. Psychopharmacology Bulletin, 21(1), 23-27.

Kelly, G.R. \& Scott, J.E. (1990). Medication compliance and health education among chronic outpatients with mental disorders. Medical Care, 28(12), 1181-1197.

Kemp, R., Kirov, G. Everitt, B., Hayward, P. \& David, A. (1998). Randomised controlled trial of compliance therapy. British Journal of Psychiatry 172, 413-419.

Kikkert, M.J., Schene, A.H., Koeter, M.W.J., Robson, D., Born, A., Helm, H. \& Gray, R.J. (2006). Medication adherence in schizophrenia: Exploring patients', carers' and professionals' views. Schizophrenia Bulletin, 32(4), 786-94.

Lacro, J.P., Dunn, L.B., Dolder, C.R., Leckband, S.G. \& Jeste, D.V. (2002). Prevalence of and risk factors for medication nonadherence in patients with schizophrenia: a comprehensive review of recent literature. Journal of Clinical Psychiatry 63(10) 892-909.

Lader, M. (1998). Pharmacological prevention of relapse. Kaohsiung Journal of Medical Science. 14(7), 448-57.

Lambert, M., Conus, P., Eide, P., Mass, R., Karrow, A. \& Moritz, S. (2004). Impact of present and past antipsychotic side-effects on attitude toward atypical antipsychotic treatment and adherence. Journal of Clinical Psychiatry, 65(9) 415-422.

Lawn, S., Smith, A. \& Hunter, K. (2008). Mental health peer support for hospital avoidance and early discharge: An Australian example of consumer driven and operated service. Journal of Mental Health, 17(5), 498-508. 
Leff, J.P. \& Wing, J.K. (1971). Trial of maintenance therapy in schizophrenia. British Medical Journal, 3 (5775), 599-604.

Leucht, S., Barnes, T.R.E., Kissling, W., Engel, R.R., Cowell, C. \& Kane, J.M. (2003). Relapse prevention in schizophrenia with new-generation antipsychotics: a systematic review and exploratory meta-analysis of randomised, controlled trials. American Journal of Psychiatry, 160(7), 1209-22.

Lewis, S. \& Lieberman, J. (2008). CATIE and CUtLASS: Can we handle the truth? The British Journal of Psychiatry, 192(3), 161-3.

Liberman, R.P. \& Kopelowicz, A. (2005). Recovery from schizophrenia: A concept in search of research. Psychiatric Services, 56(6), 735-43.

Lieberman, J.A., Stroup, S., McEvoy, J.P., Swartz, M.S., Rosenheck, R.A., Perkins, D.O., ...Hsiao, J.K. (2005). Effectiveness of antipsychotic drugs in patients with chronic schizophrenia. The New England Journal of Medicine, 353(12), 1209-1223.

Lowe, C.J., Raynor, D.K., Courtney, E.A., Purvis, J. \& Teale, C. (1995). Effects of self-medication programme on knowledge of drugs and compliance with treatment in elderly patients. British Medical Journal. 310(6989), 1129-1231.

Macpherson, R., Jerrom, B., \& Hughes, A. (1996). A controlled study of education about drug treatment in schizophrenia. British Journal of Psychiatry, 168, 709-717.

Marder, S.R. (1998). Facilitating compliance with antipsychotic medication. Journal of Clinical Psychiatry, 59(Suppl 3), 21-25

Masand, P.S. \& Narasimhan, M. (2006). Improving adherence to antipsychotic pharmacotherapy. Current Clinical Pharmacology, 1(1), 47-56.

Maxwell, J.A. (2005). Qualitative Research Design: An Interactive Approach, Second Edition, Applied Social Research Methods Series, Volume 41. California: Sage Publications.

McEvoy, J.P., Scheifler, P.L. \& Frances, A. (1999). The Expert Consensus Guideline Series: Treatment of Schizophrenia . Journal of Clinical Psychiatry, 6o(Suppl. 11), 1-80.

McGlashan, T.H., Levy, S.T. \& Carpenter, W.T. (1975). Integration and sealing over: clinically distinct recovery styles from schizophrenia. Archives of General Psychiatry, 32(10), 1269-1272.

McGorry, P.D. (1992). The concept of recovery and secondary prevention in psychotic disorders. Australian and New Zealand Journal of Psychiatry. 26(1), 3-17. 
McGrath, J., Saha, S., Welham, J, El Saadi, O., MacCauley, C. \& Chant, D. (2004). A systematic review of the incidence of schizophrenia: the distribution of rates and the influence of sex, urbanicity, migrant status and methodology. Biomedcentral Medicine, 2(13), 1741-70.

McGrath, J.J. \& Susser, E.S. (2009). New directions in the epidemiology of schizophrenia. The Medical Journal of Australia, 190(4), 7-9.

McPhillips, M.A., Kelly, F., Barnes, T.R.E., Duke, P.J., Gene-Cos, N. \& Clark, K. (1997). Detecting co-morbid substance misuse amongst people with schizophrenia living in the community: a study comparing the results of questionnaires with analysis of hair and urine. Schizophrenia Research 25(2), 141-148.

Menezes, P.R. (2009). Incidence and outcome of schizophrenia across the globe in W.F. Gattaz \& G. Busatto (Eds.), Advances in Schizophrenia Research 2009(pp.3-19). New York: Springer.

Meltzer, H.Y. (1999). Outcome in schizophrenia: beyond symptom reduction. Journal of Clinical Psychiatry, 6o(Suppl. 3), 3-7.

Mind Australia (2012). Model of recovery oriented practice. Retrieved from URL: mindaustralia.org.au

Mitchell, A.J. (2007). Adherence behaviour with psychotropic medication is a form of self-medication. Medical Hypotheses, 68(1), 12-21.

Moritz, S., Peters, M., Karow, A., Deljkovic, A., Tonn, P. \& Naber, D. (2009). Cure or curse? Ambivalent attitudes towards neuroleptic medication in schizophrenia and non-schizophrenia patients. Mental Illness, 1(1), 2

Muentz, M.R. \& Roth, L.H. (1985). Informing patients about tardive dykinesia. Archives of General Psychiatry, 42(9), 866-871.

Mueser, K. \& Gingerich, S. (2006). The Complete Family Guide to Schizophrenia. New York: The Guilford Press.

Mueser, K. \& Lewis, S. (2000). Treatment of substance misuse in schizophrenia. In P.F. Buckley \& J.L. Waddington (Eds.) Schizophrenia and Mood Disorders: The New Drug Therapies in Clinical Practice (pp. 286297.) Oxford: Reed Educational and Professional Publishing Ltd.

Noetzel, M.J., Jones, C.K. \& Conn, P.J. (2012). Emerging approaches for treatment of schizophrenia: modulation of glutamergic signalling. Discovery Medicine. 14(78), 335-43.

Novick, D., Haro J.P., Suarez, D., Perez, V., Dittann, R.W. \& Haddad, P.M. (2010). Predictors and consequences of adherence with antipsychotic medication in the outpatient treatment of schizophrenia. Psychiatry Research, 176(2-3), $109-13$. 
O'Callaghan, E., Turner, N., Renwick, L., Jackson, D., Sutton, M, Foley, S.D., ...Kinsella, A. (2010). First episode psychosis and the trail to secondary care: help-seeking and health-system delays. Social Psychiatry and Psychiatric Epidemiology, 45(3), 381-91.

O’Donnell, C., Donohoe, G., Sharkey, L., Owens, N., Migone, M., Harries, R., ... O'Callaghan, E. (2003). Compliance therapy: A randomised controlled trial in schizophrenia. British Medical Journal, 327(7419), 834.

Olfson, M., Mechanic, D., Hansell, S., Boyer, C.A., Walkup, J. \& Weiden, P.J. (2000). Predicting medication noncompliance after hospital discharge among patients with schizophrenia. Psychiatric Services, 51(2), 216-22.

Perkins, D.O., Johnson, J.L., Hamer, R.M., Zipursky, R.B., Keefe, R.S., Centorrhino, F., ...Lieberman, J.A. (2006). Predictors of antipsychotic medication adherence in patients recovering from a first psychotic episode. Schizophrenia Research, 83(1), 53-6.

Picchioni, M.M. \& Murray, R.M. (2007). Schizophrenia. British Medical Journal, 335(7610), 91-5.

Pidgeon, N. (1996). Grounded theory: Theoretical background. In J.T.E. Richardson (Ed.) Handbook of Qualitative Research Methods for Psychology and the Social Sciences (pp.75-86). Oxford: Wiley-Blackwell.

Resnick, S.G., Rosenheck, R.A \& Lehman, M.D. (2004). An exploratory analysis of correlates of recovery. Psychiatric Services. 55(5), 540-47.

Riessman, F. (1965). The "helper" therapy principle. Social Work, 1O(2), 2732.

Ritsner, M., Ponizovsky, A., Endicott, J., Nechamkin, Y., Rauchverger, B., Silver, H. \& Modai, I. (2002). The impact of side-effects of antipsychotic agents on life satisfaction of schizophrenia patients: a naturalistic study. European Neuropsychopharmacology, 12(1), 31-38.

Robinson, D., Woerner, M.G., Alvir, J.M.J., Bilder, R., Goldman, R., Geisler, S., ...Lieberman, J.A. (1999). Predictors of relapse following response from a first episode of schizophrenia or schizoaffective disorder. Archives of General Psychiatry. 56(3), 241-47.

Rogers, A. \& Pilgrim, D. (1993). Service users' views of psychiatric treatments. Sociology of Health and Illness, 15(5), 612-631.

Rogers, A., Day, J., Randall, F. \& Bentall, R.P. (2003). Patients' understanding and participation in a trial designed to improve the management of anti-psychotic medication. Social Psychiatry and Psychiatric Epidemiology, 38(12).720-727. 
Rogers, A., Day, J.C., Williams, B., Randall, F., Wood, P., Healy, D. \& Bentall, R.P. (1998). The meaning and management of neuroleptic medication: a study of patients with a diagnosis of schizophrenia. Social Science and Medicine, 47(9), 1313-23.

Rosa, M.A., Marcolin, M.A. \& Elkis, H. (2005). Evaluation of the factors interfering with drug treatment compliance among Brazilian patients with schizophrenia. Revista Brasiliera Psiquiatria, 27(3), 178-84.

Rosenstock, I.M. (1974). Historical origins of the health belief model. Health Education Monographs, 2(4), 328.

Rubin, H.J. \& Rubin, I.S. (1995). Qualitative Interviewing: The Art of Hearing. London: Sage.

Schimmelmann, B.G., Huber, C.G., Lambert, M., Cotton S, McGorry, P.D. \& Conus, P. (2008). Impact of duration of untreated psychosis on pretreatment, baseline, and outcome characteristics in an epidemiological firstepisode psychosis cohort. Journal of Psychiatric Research 42(12), 982-90.

Schneider, B. (2010). Hearing (our) voices: participatory research in mental health. Canada: University of Toronto Press.

Schooler, N.R. (2004). Maintenance medication for schizophrenia:

Strategies for dose reduction. Focus, 2(1), 175-86.

Schulz, C. \& McGorry, P. (2000). Traditional antipsychotic medications: contemporary clinical use. In P.F. Buckley \& J.L. Waddington (Eds.)

Schizophrenia and Mood Disorders: The New Drug Therapies in Clinical Practice (pp.14-20) Oxford: Reed Educational and Professional Publishing Ltd.

Sharif, Z., Bradford, D., Stroup, S. \& Lieberman, J. (2007). Pharmacological treatment of schizophrenia. In P.E. Nathan \& J.M. Gorman (Eds.) A Guide to Treatments that Work (Third Edition) (pp. 203-243). New York. : Oxford University Press

Sharif, S.A., Ogunbanjo, G.A. \& Malete, N.H. (2003). Reasons for noncompliance to treatment amongst patients with a psychiatric illness. South African Family Practice. 45(4), 10-13.

Shean, G. (2004). Understanding and Treating Schizophrenia:

Contemporary Research, Theory and Practice. Michigan: Haworth Clinical Practice Press

Shoemaker, S.J. \& Ramalho de Oliveira, D. (2008). Understanding the meaning of medications for patients: the medication experience. Pharmacy, World and Science. 30(1), 86-91. 
Smith, T.E., Weston, C.A. \& Lieberman, J.A. (2009). Schizophrenia (maintenance treatment). Clinical Evidence, 82(4), 338-339.

Sperry, L. (1995). Psychopharmacology and Psychotherapy: Strategies for Maximizing Treatment Outcomes. New York: Brunner Mazel Inc.

Svarstad, B.L., Shireman, T.I. \& Sweeney, J.K. (2001). Using drug claims data to assess the relationship of medication adherence with hospitalisation and costs. Psychiatric Services, 52(6), 805-811.

Swaminath, G. (2007). You can lead a horse to the water.... Indian Journal of Psychiatry, 49(4), 228-30.

Torrey, E.F. (2011). Patients, clients, consumers, survivors et al: what's in a name? Schizophrenia Bulletin. 37(3), 466-8.

Valenstein, M., Blow, F.C., Copeland, L.A., McCarthy, J.F., Zeber, J.E., Gillon, L., ...Stavenger, T. (2004). Poor Antipsychotic Adherence Among Patients With Schizophrenia: Medication and Patient Factors. Schizophrenia Bulletin, 3o(2), 255-64.

Van Os, J. \& Kapur, S. (2009). Schizophrenia. The Lancet, 374(9690), 63545 .

Voruganti, L.P., Heslegrave, R.J. \& Awad, A.G. (1997). Neuroleptic dysphoria may be the missing link between schizophrenia and substance misuse. Journal of Nervous and Mental Disease 185(7), 463-5.

Wciorka, J. (1988). A clinical typology of schizophrenic patients' attitudes towards their illness. Psychopathology, 21(6), 259-66.

Weiden, P.J.(1991). Neuroleptic non-compliance in schizophrenia. In C. Tamminga and S.C. Schulz (Eds) Advances in Neuropsychiatry and Psychopharmacology (pp.285-95). New York: Raven Press.

Weiden, P.J \& Glazer, W. (1997). Assessment and treatment selection for "revolving door" inpatients with schizophrenia. Psychiatric Quarterly. 68(4), 377-92.

Weiden, P.J., Rapkin, B., Mott, T., Zygmunt, A., Goldman, D., HorvityzLennon, M. \& Frances, A. (1994). Rating of Medication Influences (ROMI) scale in schizophrenia. Schizophrenia Bulletin, 20(2), 297-310.

Weiden, P.J., Scheifler, P.L., Diamond, R.J. \& Ross, R. (1999). Breakthroughs in Antipsychotic Medications: A Guide for Consumers, Families, and Clinicians. New York: The National Alliance for the Mentally Ill.

Willig, C. (2001). Introducing qualitative research in psychology: Adventures in theory and method. Philadelphia: Open University Press. 
Wise, J.W. (2004). Mad science: discourses of 'schizophrenia' and 'therapy' for hearing voices. (Unpublished doctoral dissertation). Murdoch University, Perth, Western Australia, Australia.

Xiang, M., Ran, M. And Li, S. (1994). A controlled evaluation of psychoeducational family intervention in a rural Chinese community. British Journal of Psychiatry, 165(4), 544-8.

Xiong, W., Phillips, M.R., Hu, X., Wang, R., Dai, Q., Kleinman, J. \& Kleinman, A. (1994). Family based intervention for schizophrenic patients in China. A randomised controlled trial. British Journal of Psychiatry, 165(2), 239-47.

Yang, J., Ko, Y.H., Paik, J.W., Lee, M-S., Han, C., Joe, S-H., ...Kim, S-H. (2011). Symptom severity and attitudes towards medication: impacts on adherence in outpatients with schizophrenia. Schizophrenia Research, 134(2), 226-231.

Zygmunt, A., Olfson, M., Boyer, C.A. \& Mechanic, D. (2002). Interventions to improve medication concordance in schizophrenia. American Journal of Psychiatry, 159(10), 1653-64. 


\section{INTERESTED PEOPLE WANTED FOR LOCAL RESEARCH!}

Do you.....

- Have Schizophrenia?

- Take antipsychotic medication?

- Understand and communicate well in English?

If so, you are invited to participate in a study.

\section{What is the study about?}

The research team is interested in finding out about your experiences of taking medication.

\section{What is involved?}

If you choose to take part, you will be involved in a one-to-one interview with a researcher. You will be asked about your experiences of medication taking and what strategies you believe could be helpful in improving medication taking amongst people with Schizophrenia. The interview should take no longer than 1 hour to complete and refreshments will be provided. You do not have to answer all questions and are free to withdraw at any time.

\section{Interested? Who to Contact:}

If you think that you may be interested in taking part in this study then you should contact the primary researcher of the studyLucinda on XXXX XXX XXX. Alternatively, you may wish to contact Bev Hisee on XXXX XXX XXX or XXXX XXXX, or Dr. Cherrie Galletly on XXXX XXXX for more information about the study and to answer any questions that you may have. If, after gaining more information, you remain keen to take part a time can then be arranged for the session to occur. 


\section{Appendix B Interview Schedule}

- Tell me about your experiences of taking antipsychotic medication.

- Tell me about times when you have taken your antipsychotic medication consistently.

- Tell me about times when you have not taken your antipsychotic medication consistently.

- What influences you to take/not take your antipsychotic medication?

- What are some of the benefits associated with taking your antipsychotic medication?

- What are some of the negative aspects associated with taking your antipsychotic medication/things that might make you want to stop?

- What are some incentives to taking your antipsychotic medication?

- What are some barriers to taking your antipsychotic medication? 


\section{Appendix C Information Sheet}

\section{है। \\ LYELL MCEWIN HEALTH SERVICE \\ Mental Health Division \\ PARTICIPANT INFORMATION SHEET}

TITLE

"Medication Concordance amongst People with Schizophrenia"

\section{INVITATION TO PARTICIPATE}

I am a postgraduate student in Psychology inviting you to participate in a research project which my supervisors and I believe is of potential importance. In order to participate in the study, you must be between the ages of 18 and 65 , have a diagnosis of schizophrenia and be taking antipsychotic medication. Before you decide whether or not you wish to participate, we need to be sure that you understand the following:

Why we are doing it, and

What it would involve if you agreed.

We are giving you the following information. Please read it carefully and be sure to ask any questions you have; the researchers conducting the study will be happy to discuss them with you. You are also free to discuss the study with other people if you wish. (ie. family, friends and / or your local Doctor).

You do not have to make an immediate decision.

Your participation is purely voluntary, and you are under no pressure to participate.

Should you agree to take part, you may change your mind and withdraw at any stage.

Please ask the research staff to explain any words or information contained in this form that you do not understand clearly.

\section{WHAT IS THE STUDY ABOUT?}

I am interested in how people with schizophrenia feel about taking antipsychotic medications everyday and their reasons for taking or not taking their medication. We are inviting you to help us leam about your experiences of antipsychotic medications.

\section{WHAT HAPPENS DURING THE STUDY?}

If you choose to take part in the study, you will be interviewed about your beliefs about antipsychotic medication. You may also be asked for some advice about how to encourage people with schizophrenia to take their medication. The interview will take about one hour. All one-to-one interviews will be held in interview rooms at community centres or Mental Ilness Fellowships. We can organise a time that suits both of us. You do not have to respond to all questions and may end the interview at any time.

WHO WILL MANAGE MY TREATMENT IF I TAKE PART?

Your usual treatment team will manage your treatment during the study.

WHAT ARE THE DISCOMFORTS, RISKS AND SIDE EFFECTS?

This study does not involve the use of drugs or medical procedures.

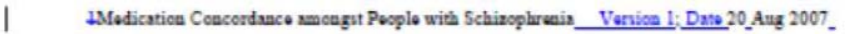




\section{WHAT WILL HAPPEN TO THE INFORMATION COLLECTED?}

Interviews will be audio recorded and later, typed up. The interview information will be used to set up an intervention which will aim to encourage medication taking amongst people with schizophrenia.

Your participation will remain confidential at all times. Your name or anything that could identify you will not appear in any report or record of the study. At the end of the study, if you wish, you can view my research findings.

\section{WHAT WILL I GET OUT OF THE STUDY?}

It is unlikely that you will gain anything from this study.

\section{WHAT ARE MY RIGHTS?}

If you become injured during this study, Lyell McEwin Health Service will provide reasonable medical treatment. If you become distressed, your case manager or ACIS will be contacted. Your participation in this study shall not affect any other right to compensation you may have under common law.

If at any time you have a question about this study or want more information you may contact Lucinda Clifford, phone: 1 email: You may also contact Prof. Cherrie Galletly, phone:
Prof. Deborah Tumbull, phone: email:
; or Dr. Shona Crabb - ph: email:

Participation in this study is entirely voluntary. You are free to refuse to take part or to withdraw from the study at any time without having to give a reason and without this affecting your future medical care or your relationships with medical staff looking after you.

\section{WHAT IF I HAVE A QUESTION ABOUT THE STUDY?}

The Central Northern Adelaide Health Service Ethics of Human Research Committee has approved this study. Should you wish to speak to a person not directly involved in the study in relation to:

- matters conceming policies,

- information about the conduct of the study

- your rights as a participant, or

- should you wish to make a confidential complaint, you may contact the Executive Officer of this Committee on 0882226841 . 


\title{
Appendix D Consent Form
}

\author{
(5) \\ LYELL MCEWIN HEALTH SERVICE \\ Mental Health Division
}

Consent Form

1. I, the undersigned

hereby consent to my involvement in the research project titled

2. I have read the information sheet, and I understand the reasons for this study. The ways in which it will affect me have been explained by the research worker. My questions have been answered to my satisfaction. My consent is given voluntarily.

3. The details of the research project have been explained to me, including:-

- the expected time it will take

- the nature of any procedures being performed, and the number of times they will be performed

- any risks/discomforts which I may experience

4. I understand that the purpose of this research project is to improve the quality of medical care, but my involvement may not be of benefit to me.

5. I have been given the opportunity to have a member of family or a friend present while the project was explained to me.

6. My identity will be kept confidential, and nothing will be published which could possibly reveal my identity.

7. My involvement in the project will not affect my relationship with my medical advisers. I understand I am free to withdraw from the project at any stage without having to give any reasons, and that if I do withdraw from the project it will not affect my treatment at this hospital in the future.

SIGNED

ADDRESS

(please print)

WITNESS

RESEARCH WORKER

DATE 


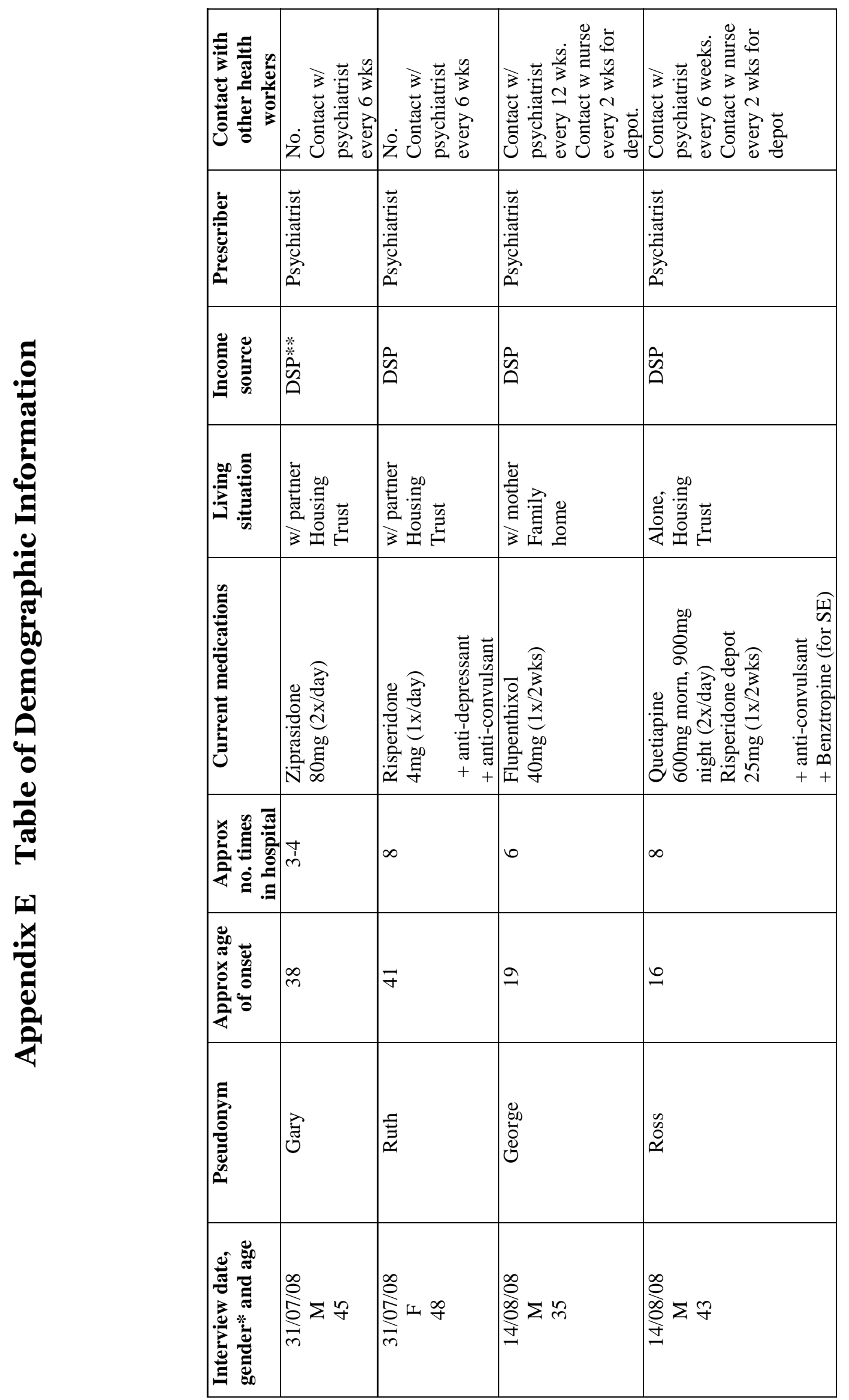

굼 


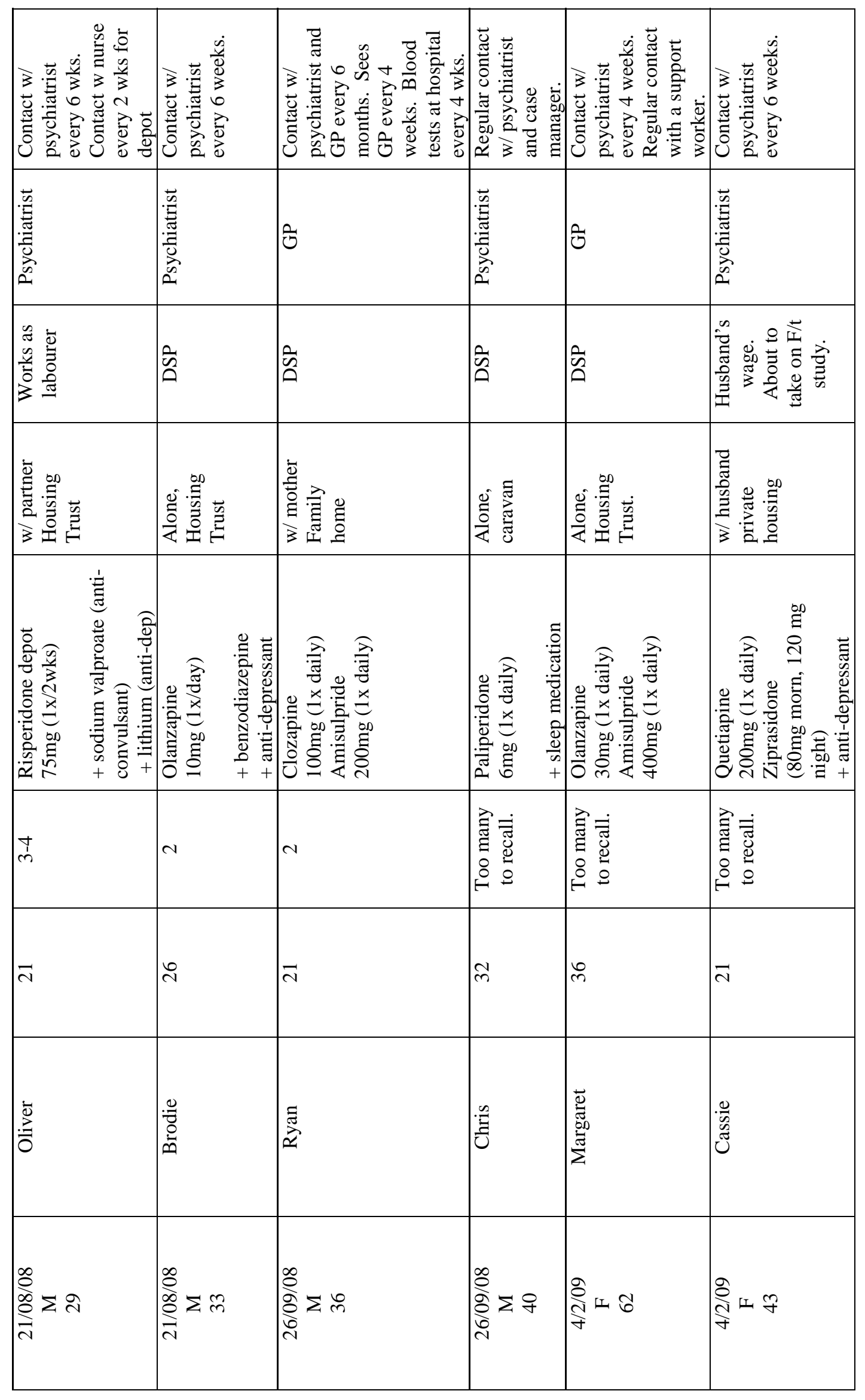

$\stackrel{\infty}{m}$ 


\begin{tabular}{|c|c|c|c|c|c|}
\hline 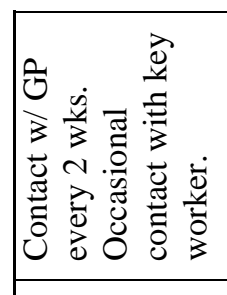 & 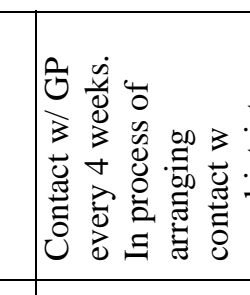 & 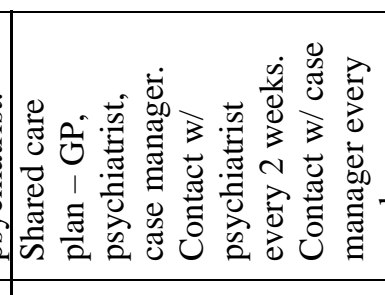 & 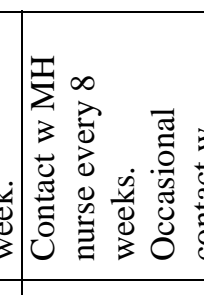 & 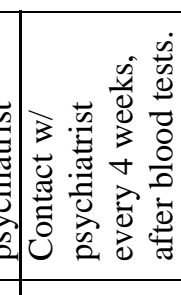 & 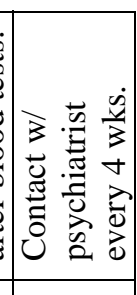 \\
\hline 今ि & 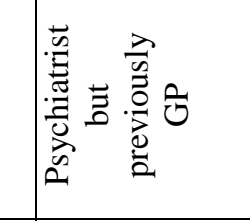 & 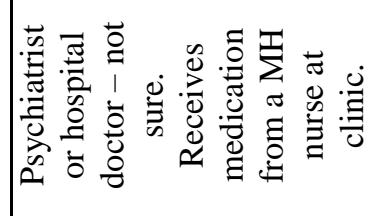 & 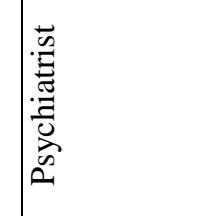 & 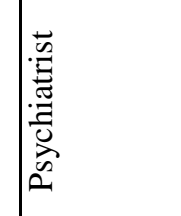 & 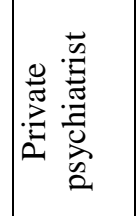 \\
\hline हิે & 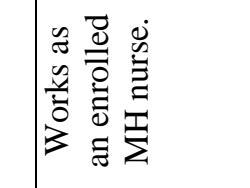 & 茼 & 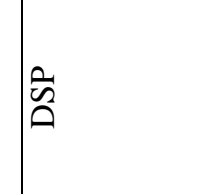 & 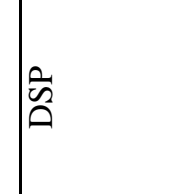 & 苂 \\
\hline 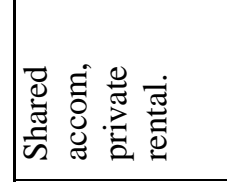 & 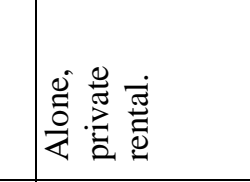 & 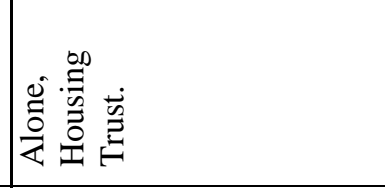 & 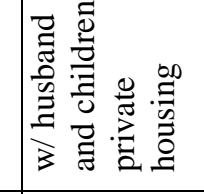 & 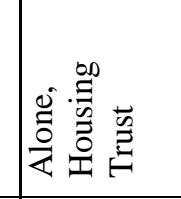 & 焉 \\
\hline 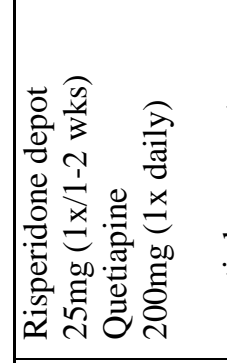 & 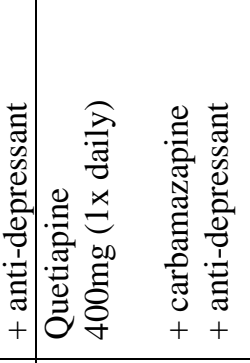 & 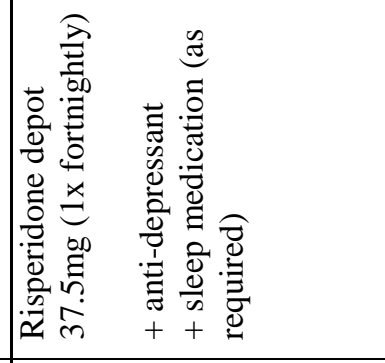 & 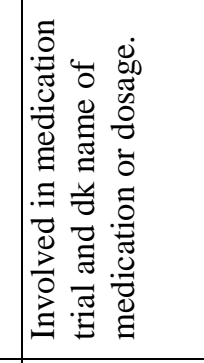 & 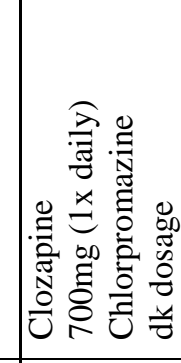 & $\begin{array}{ll}0 \\
0\end{array}$ \\
\hline$\underline{\underline{e}}$ & + & œ & $\theta$ & in & ब \\
\hline$\stackrel{\sim}{\sim}$ & 㚈 & 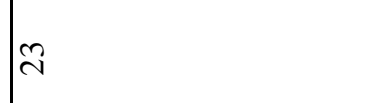 & চি & $\nexists$ & $\pi$ \\
\hline 峁 & $\mid$ & 定 & 䓀 & 鹿 & 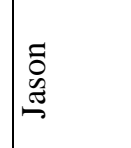 \\
\hline & 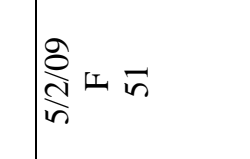 & 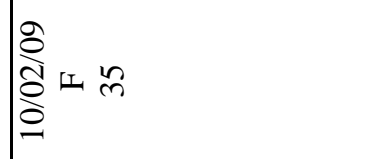 & 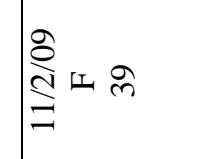 & 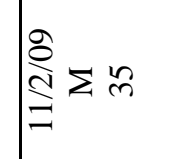 & \\
\hline
\end{tabular}




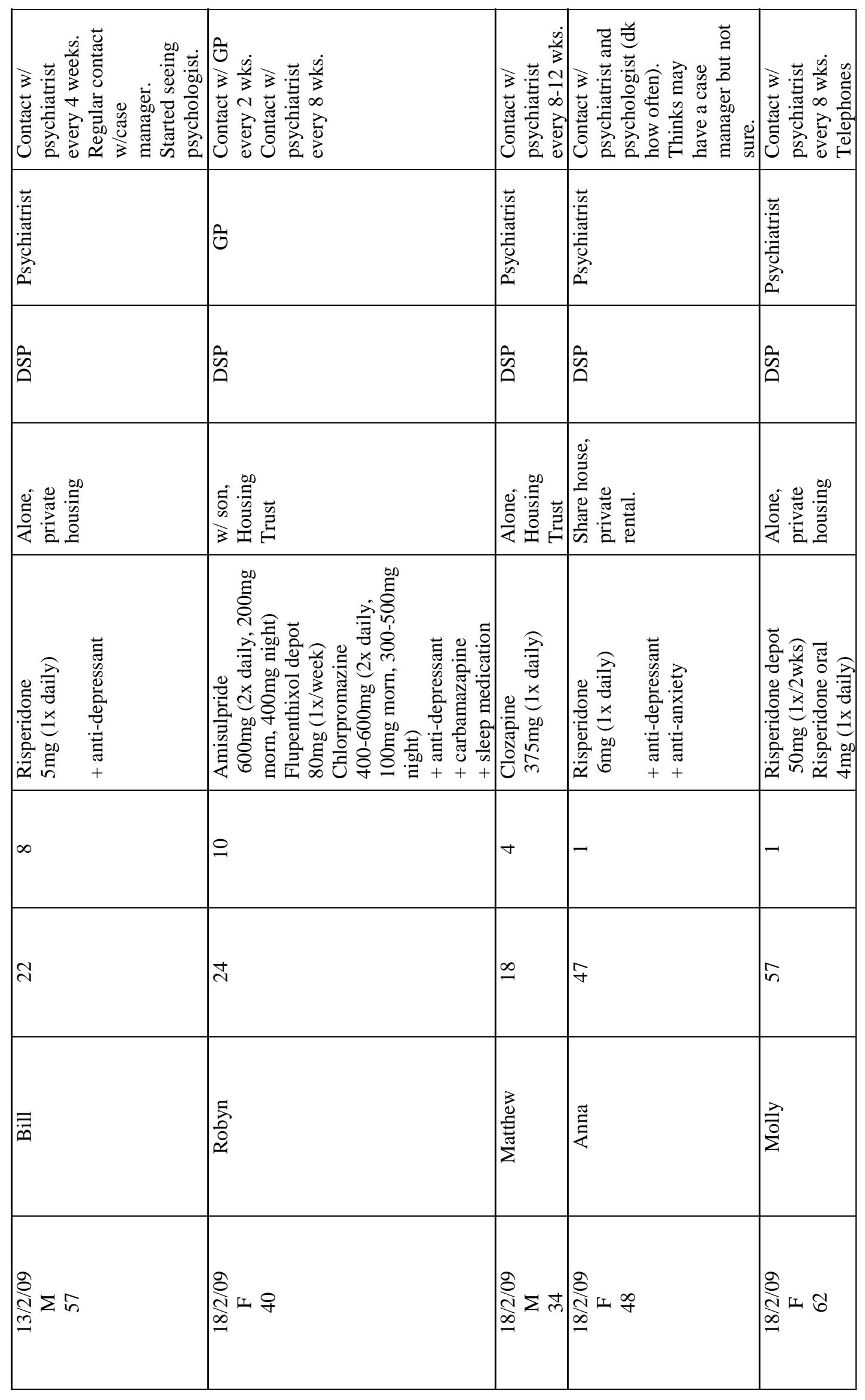




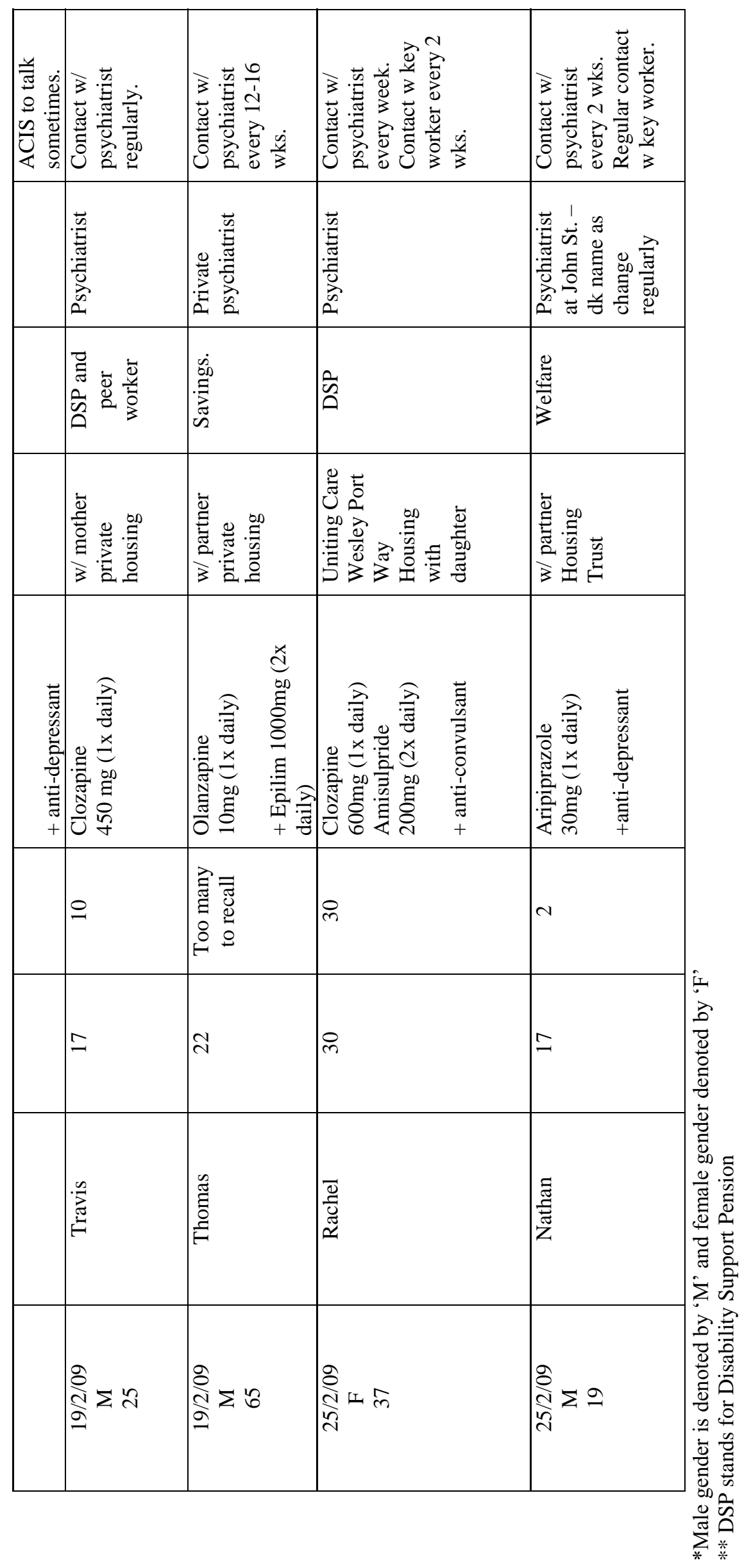

च 